\title{
Autonomous and non-autonomous requirements for the c-Jun N- terminal kinase signaling pathway in early forebrain development
}

Jessica G. Cunningham

West Virginia University, jgclemente@mix.wvu.edu

Follow this and additional works at: https://researchrepository.wvu.edu/etd

Part of the Neurosciences Commons

\section{Recommended Citation}

Cunningham, Jessica G., "Autonomous and non-autonomous requirements for the c-Jun N-terminal kinase signaling pathway in early forebrain development" (2022). Graduate Theses, Dissertations, and Problem Reports. 7739.

https://researchrepository.wvu.edu/etd/7739

This Dissertation is protected by copyright and/or related rights. It has been brought to you by the The Research Repository @ WVU with permission from the rights-holder(s). You are free to use this Dissertation in any way that is permitted by the copyright and related rights legislation that applies to your use. For other uses you must obtain permission from the rights-holder(s) directly, unless additional rights are indicated by a Creative Commons license in the record and/ or on the work itself. This Dissertation has been accepted for inclusion in WVU Graduate Theses, Dissertations, and Problem Reports collection by an authorized administrator of The Research Repository @ WVU.

For more information, please contact researchrepository@mail.wvu.edu. 


\title{
Autonomous and non-autonomous requirements for the C-Jun $\mathrm{N}$-terminal kinase signaling pathway in early forebrain development
}

\author{
Jessica Genevieve Cunningham \\ Dissertation submitted to the School of Medicine \\ at West Virginia University \\ In partial fulfillment of the requirements for the degree of \\ Doctor of Philosophy in \\ Neuroscience
}
Eric Tucker, Ph.D., Chair
Peter Mathers, Ph.D.
Ariel Agmon, Ph.D.
Candice Brown, Ph.D.
Sadie Bergeron, Ph.D.

Neuroscience Graduate Program

Morgantown, WV

2020

\begin{abstract}
Keywords: neural development, c-Jun N-Terminal Kinase (JNK), forebrain, cortical interneurons, thalamocortical axons
\end{abstract}

Copyright 2020 Jessica Genevieve Cunningham 


\title{
Abstract \\ Autonomous and non-autonomous requirements for the c-Jun $\mathrm{N}$-terminal kinase signaling pathway in early forebrain development
}

\author{
Jessica Genevieve Cunningham
}

The cerebral cortex is responsible for a wide variety of high-level functions including cognition, sensory perception, fine motor control, and the orchestration of body movements. The cortex is comprised of cortical excitatory neurons and inhibitory interneurons, which are arranged in a highly organized fashion into different layers and regions. These two types of cells operate in a delicate balance between excitation and inhibition, which is critical for proper cortical circuitry. In order for the cortex to execute its numerous functions, it must both send and receive input to other brain regions through axonal connections. The organization within the cortex and orchestration of connections with other brain regions is established from very early in development, and disruptions occurring even during embryogenesis can lead to lasting changes in cortical circuitry. Neurodevelopmental disorders such as autism, epilepsy, and schizophrenia are thought to arise from disturbances in the formation of cortical circuits, which can occur years before a disease physically manifests. Therefore, it is critical to understand the fundamental mechanisms responsible for early circuitry formation in order to gain better insight into the causes of these diseases.

This dissertation explores the role of the c-Jun N-terminal kinase (JNK) signaling pathway in early forebrain development. A novel genetic knockout mouse model is used to eliminate all of JNK signaling in vivo from a population of cells that gives rise to cortical inhibitory interneurons. In Chapter 2, I provide evidence that JNK signaling is required for the proper migration of interneurons during embryonic development and their correct laminar allocation in the early postnatal cortical wall. This is demonstrated through both in vivo genetic approaches and ex vivo pharmacological inhibition of JNK signaling, and utilizes live-imaging techniques to assess the dynamic properties of migratory interneurons. In Chapter 3, I discovered a novel, non-autonomous requirement for JNK signaling in the pathfinding of thalamocortical axons. When JNK signaling is eliminated in the ventral telencephalon, it causes a misrouting of the thalamocortical axons that normally traverse through this territory. These are the first studies examining the complete loss of JNK function from cells located in the forebrain in vivo, and provide novel insight into the roles of JNK signaling in the development of cortical inhibitory interneurons and thalamocortical axons. Understanding the genetic regulation of forebrain development will help uncover potential causes of neurodevelopmental disorders, and can ultimately lead to better treatment of these devastating diseases. 


\section{Acknowledgements}

To my principal investigator and mentor Dr. Eric Tucker, thank you for everything you have done for me over the past several years. Little did I know that I would find my PhD laboratory back in the Spring of 2014 in Medical Neurobiology, nor could I have anticipated all that I have learned over the entire course of my PhD work. I appreciate your patience with me as you allowed me to grow into the scientist that I am today, and for teaching me different laboratory techniques, instructing me on how to put together a meticulous poster or talk, and facilitating my attendance at national conferences.

To my thesis committee, thank you for insightful feedback on my projects, talks, papers, and dissertation. You have been a wonderful resource and I appreciate you taking the time to share your expertise.

To all of my mentors during my adolescence, I would not have chosen this career path if it were not for you believing in me before I believed in myself. Dr. Dahms, Mrs. Timposon, and Mrs. Wentzel, thank you for fostering my love of science in high school Chemistry and Biology classes. Dr. Skorpinski, thank you for motivating me to pursue this dual degree program from before I even knew it existed.

To Dr. Jack Williams, you ignited a fire of curiosity in me and a true love and passion for research. I will always remember our days at Mercyhurst troubleshooting SPME, learning the art of brewing coffee, traveling to Atlanta for my fist national conference, and publishing my first paper.

To Dr. Christina Barr, Dr. David Goldman, Stephen Lindell, and all of my wonderful colleagues in the Laboratory of Neurogenetics at the NIH-NIAAA, you provided an environment for me to grow and develop as a scientist, and helped solidify my desire to pursue a career in scientific research.

To my family, your unwavering support has meant the world to me. Mom, Dad, Jonathan, and Jillian, you have provided unconditional love and support from our early days of deer teeth science fair projects, and are always available for whatever help I need. Chris, you have certainly earned your honorary spouse PhD. You have kept me grounded through this process, and I could not have asked for a better life partner. Thank you for all of your sacrifices so I can pursue my career ambitions, and for tolerating my special support staff of the cat and Mr. Penguin.

To all of my lab mates, what a journey it has been! Skye, Catherine, and James, it is going to be difficult to say "byeee," but I would not have wanted to complete my PhD with anyone else but you (even if you are wearing that Patriots shirt James). Thank you to all of my former lab members including Abby, Jawuanna, Katie, Kelly, Melissa, and Kareem, who each taught me something new along my journey, and to Steven for keeping me sane and "work excited" during your time in the lab. A special thank you to Stephany for all of your dedication to our research- your motivation and work ethic is truly inspiring, and I am so excited to see where your journey will take you.

To all of the support staff at WVU, you have helped me in both my scientific endeavors and also helped me grow as a leader. Mandy and Karen, thank you for providing endless assistance with microscopy. Erica, Morgan, and Cassie, you all do so much for the Neuroscience program and its students, and your behind-the-scenes work does not go unnoticed. Dr. Jen, Dr. Gibson, Dr. Salati, Dr. Lockman, Joan, Nicole, and Connor, thank you so much for all of your help with GWAS and for your guidance in helping me develop into the leader I am today.

To all of my friends who have become family, my journey would not have been the same without each of you in it. From becoming friends with Lauryn on our MD/PhD interview, to fortuitously meeting Sylwia in my first lab rotation, I have developed friendships that will last a lifetime. Emily, Tayvia, and Abi you have all been a wonderful support system during trying times in school and the lab, and I am so happy you stood by my side on my wedding day. Neal it has been a blast hanging out to share a drink and spend time with the cat, and Rachael it has been so much fun getting to know you and working with you to grow GWAS. Thank you to all of my friends who have supported me along this long journey from both near and far! 


\section{Table of Contents}

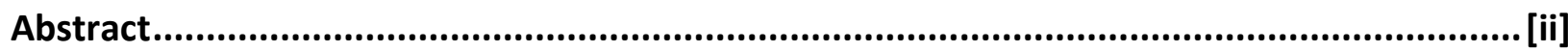

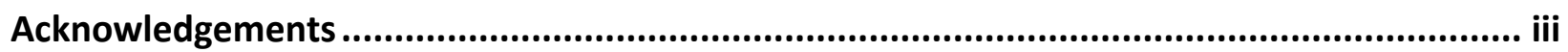

Table of Contents ...................................................................................................................... iv

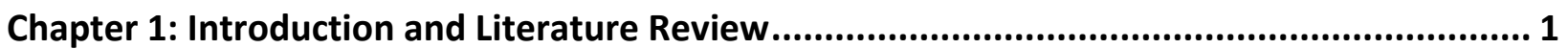

The cerebral cortex ....................................................................................................... 2

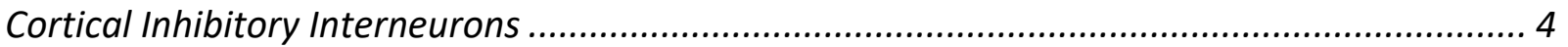

Overview of properties and functions.......................................................................... 4

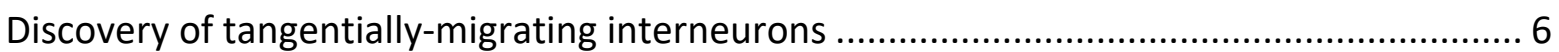

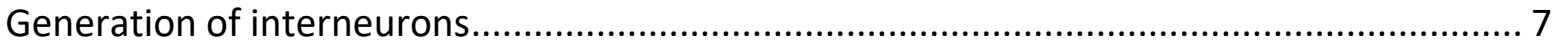

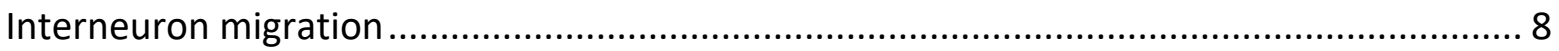

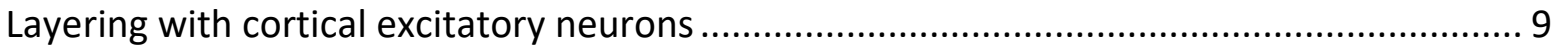

Cortical circuitry diseases and development................................................................ 11

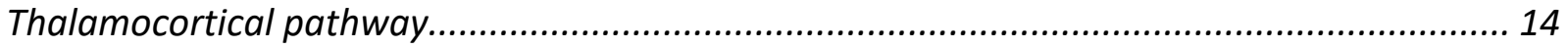

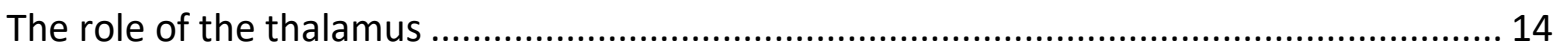

Thalamocortical connectivity and neurodevelopmental disorders .................................... 16

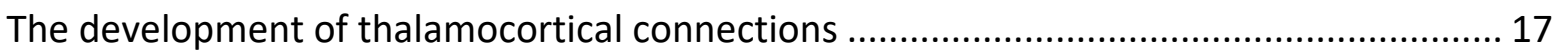

Thalamocortical axon repulsion from the hypothalamus ................................................... 18

Thalamocortical axon crossing at the diencephalon-telencephalon boundary (DTB).......... 19

Navigation of thalamocortical axons through the telencephalic corridor ............................ 21

Sorting of thalamocortical axons in the ventral telencephalon ......................................... 22

Longitudinal axon pathways that cross the DTB in early development............................... 23

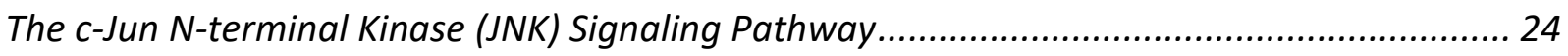

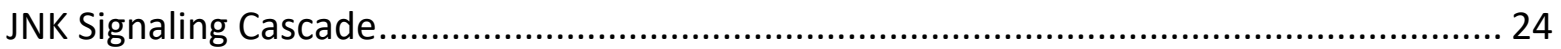

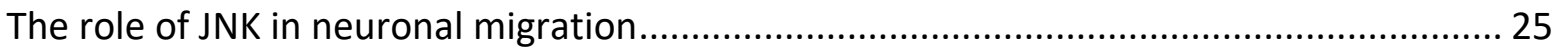

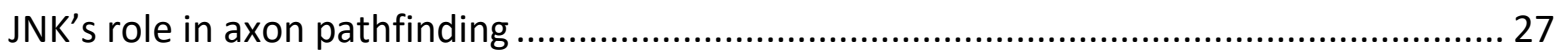

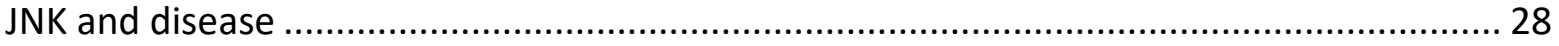

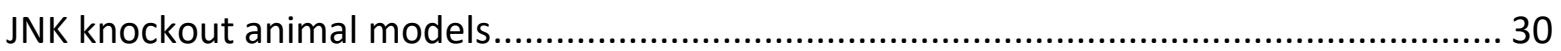

Chapter 2: JNK signaling is required for proper tangential migration and laminar allocation of

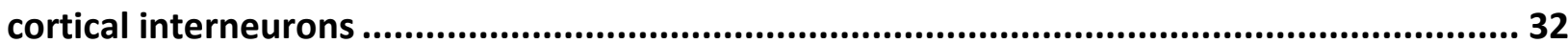

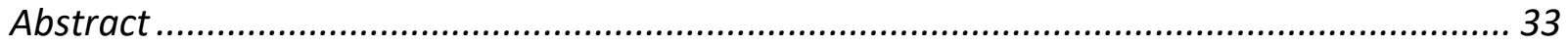

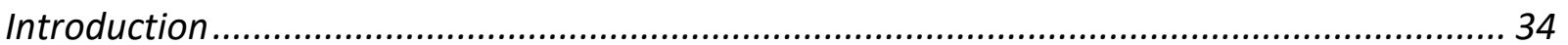

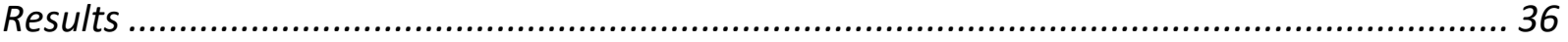


Loss of JNK signaling alters the radial distribution and migratory orientation of cortical interneurons

JNK inhibition disrupts migratory properties of cortical interneurons and causes premature departure from the SVZ stream........................................................................... 37

Migratory stream integrity can be partially restored after removal of JNK inhibition ......... 38 JNK signaling is required for interneurons to enter the cortex at the correct time in vivo.. 40 Genetic loss of JNK signaling alters interneuron distribution and morphology .................. 41 Genetic removal of JNK alters the dynamic migratory properties of cortical interneurons in ex vivo slices 42

JNK-depleted interneurons are incorrectly distributed in the early postnatal cortex......... 44 Assessing autonomy for JNK function in interneuron migration and allocation ................ 45

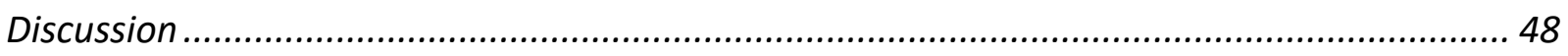

Molecular mechanisms underlying cortical plate invasion by interneurons ...................... 48

Diverse requirements for JNK during cortical interneuron migration .............................. 50

Conclusions and Future Perspectives ............................................................................. 52

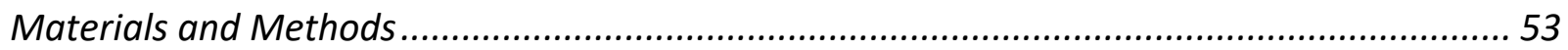

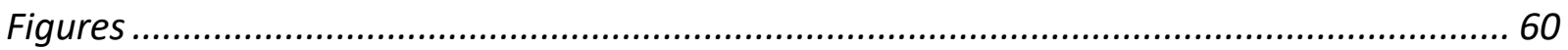

Chapter 3: A non-autonomous requirement for JNK signaling in early thalamocortical axon

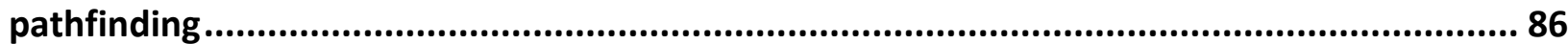

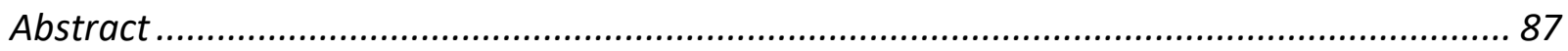

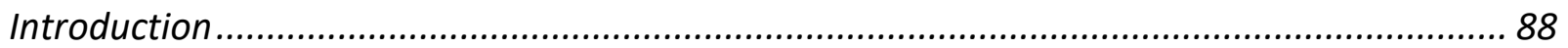

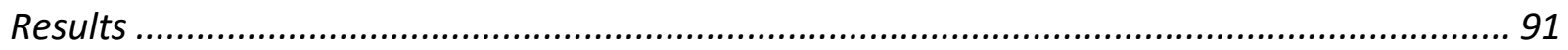

Axon tracts are missing or misrouted in CTKO mice........................................................ 91

Thalamocortical axons misroute ventrally in cTKO mice ............................................ 92

Thalamocortical and corticothalamic axons take aberrant trajectories in cTKO mice .........93

Thalamocortical axons are misrouted early in development in cTKO brains ...................... 95

Telencephalic corridor and guidepost cells are disrupted in cTKO mice ........................... 96

Reciprocal connections between the striatum and substantia nigra are altered in cTKO mice . 98

Non-autonomous requirement for JNK signaling in thalamocortical axon pathfinding ....... 99

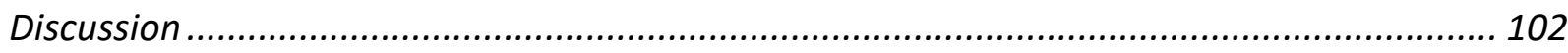

Autonomous and non-autonomous requirements for JNK signaling in the development of major forebrain and midbrain axon trajectories............................................................... 102

Non-canonical WNT signaling in thalamocortical axon extension ................................... 104

Axon guidance cues and gradients in thalamocortical axon pathfinding ........................ 106

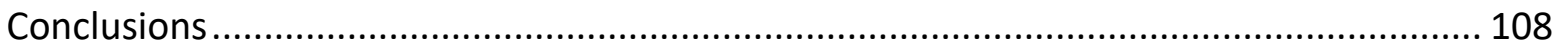

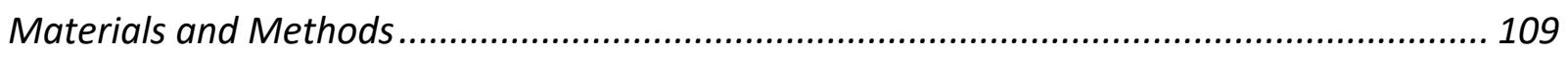


Figures

Chapter 4: Summary and Future Directions ..................................................................... 139

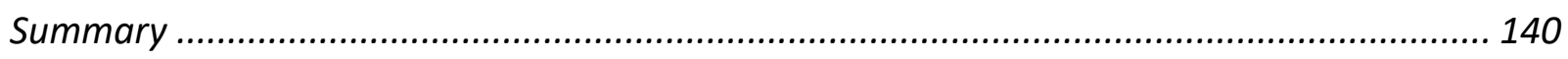

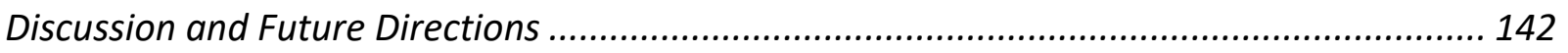

Inhibitory interneurons ................................................................................... 142

Cortical excitatory neurons and radial glia............................................................. 144

Thalamocortical influence on interneuron migration ............................................... 146

Attractive and repulsive cues for guiding thalamocortical axons .................................. 148

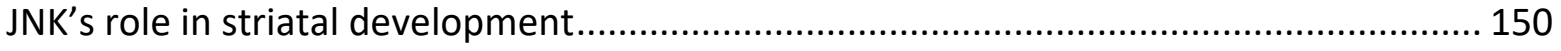

Potential autonomous requirement for JNK in thalamocortical axons ............................ 153

The possible influence of glial cells on thalamocortical axon pathfinding ......................... 154

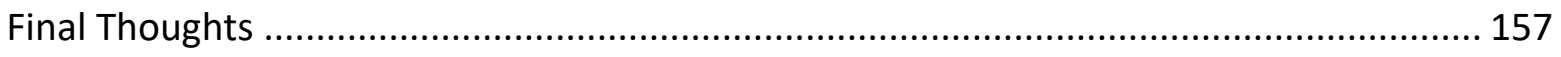

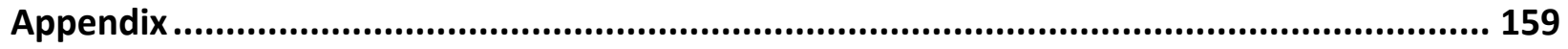

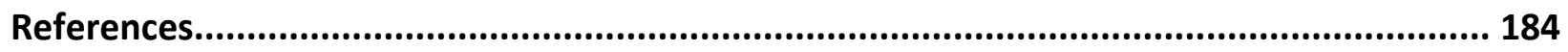


Chapter 1: Introduction and Literature Review 


\section{The cerebral cortex}

The cerebral cortex is a highly organized structure that is responsible for a wide variety of functions including cognition, movement, learning, and language (Misic et al., 2016). The cortex can be divided into two hemispheres, each of which is organized into the frontal, parietal, temporal, occipital, and limbic lobes (Broca, 1878; Leuret and Gratiolet, 1839; Pessoa and Hof, 2015). Anatomical regions that are histologically distinct can be defined by specialized structure and function, which are referred to as Brodmann's areas (Brodmann, 1909). Within the cortex itself, the grey matter contains both glutamatergic excitatory neurons and $\mathrm{y}$-aminobutyric acidergic (GABAergic) inhibitory interneurons, which function together in a delicate balance (Tatti et al., 2017). These two cell types are histologically organized into horizontal layers and vertical columns, which was first observed over a century ago through Golgi staining of the cortex by Ramon y Cajal (Ramon y Cajal, 1881). The radial columns were first shown to be functionally related in the somatosensory cortex of a cat (Mountcastle, 1957), and have subsequently been studied to gain a deeper understanding of the relationship between cytoarchitecture and resultant function (Jones and Rakic, 2010; Rakic, 2007).

Even though inhibitory interneurons and excitatory neurons eventually occupy the same cortical laminae in the cortical wall, their developmental origins and migratory routes are very distinct (Nadarajah and Parnavelas, 2002; Wichterle et al., 2001). Inhibitory interneurons are born in subcortical locations, and migrate tangentially over a long distance to reach their final laminar position (Hatanaka et al., 2016; Wichterle et al., 2001). In contrast, excitatory neurons are born in the cortex itself, and migrate radially over a relatively short distance before depositing into the correct cortical layer (Rakic, 1972; Tan and Shi, 2013). In the developing cortex, neural progenitors are located in the ventricular zone (VZ) and subventricular zone (SVZ) and undergo an asymmetric division to give rise to cortical excitatory neurons (Miyata et al., 2004; Williams and Price, 1995). Excitatory neurons then migrate along radial glia scaffolding, which have cell bodes in the VZISVZ and processes that extend to the pial surface 
of the cortical wall, and are actually themselves neural progenitors (Campbell and Gotz, 2002; Levitt and Rakic, 1980; Noctor et al., 2001). Excitatory neurons are known to deposit in an "inside-out" fashion, in which early-born neurons deposit into the lower layers of the developing cortical plate, and late-born neurons deposit into upper layers. This was first discovered by radioactive thymidine injections in the developing mouse (Angevine and Sidman, 1961), and later in rats (Berry and Rogers, 1965) and monkeys (Rakic, 1977). The correct migration of both excitatory and inhibitory neurons throughout development, and subsequent organization into a six-layered neocortex, are critical for the functional capabilities of the human cortex later in life (Misic et al., 2016).

While certain brain functions are primarily executed by one structural region, it is believed that complex behaviors as well as cognitive functions emerge from interactions between different brain areas (Cristofori et al., 2019; McIntosh, 2000). White matter consists of axon tracts that serve to connect the cortex to both itself and other brain regions (Sherman and Guillery, 2011). Nearly all cortical regions send projections to and receive input from the thalamus, a centralized brain structure containing dozens of nuclei that relays important motor and sensory information to distinct cortical regions via thalamocortical axons (Adams et al., 1997; Gezelius and Lopez-Bendito, 2017). These connections are critical for proper circuitry function, and also allow for higher-order processing of information within a particular cortical region or across different cortical areas (Nakajima and Halassa, 2017; Sherman, 2017). Disruptions to the formation of cortical circuits, even those that occur during embryogenesis, can lead to neurodevelopmental disorders such as autism, epilepsy, and schizophrenia (Doherty and Owen, 2014; Kato and Dobyns, 2005; Rossignol, 2011). It is therefore critical to gain a deeper understanding of the early developmental processes involved in cortical circuitry formation. 


\section{Cortical Inhibitory Interneurons}

\section{Overview of properties and functions}

Cortical inhibitory interneurons are greatly outnumbered by cortical excitatory neurons, representing around $20 \%$ of all neurons in the cerebral cortex (Beaulieu et al., 1992; Hendry et al., 1987; Meinecke and Peters, 1987). Interneurons form inhibitory connections onto nearby neurons, as well as establish gap junction-mediated electrical networks with other interneurons, which can serve to regulate excitatory transmission produced in the brain (Markram et al., 2004). Various populations of interneurons can even work simultaneously to provide distinct input frequencies to different regions of pyramidal neurons (Whittington and Traub, 2003). These connections are formed through synapses, which permit the direct transmission of electrochemical signals between cells, allowing inhibitory interneurons to coordinate the activity of other cortical cell populations (Hestrin and Galarreta, 2005).

Even though interneurons are a minority of cells in the cerebral cortex, they play many essential roles in various types of cortical function. In the visual cortex, interneurons induce critical periods in cortical plasticity, which is necessary for proper visual function (Hensch, 2005). The critical period can actually be delayed or brought forward by altering GABA transmission, and reduced inhibition in early life in a mouse model prevented experiencedependent plasticity (Fagiolini and Hensch, 2000; Iwai et al., 2003; Morales et al., 2002). In the auditory cortex, interneurons were shown to facilitate associative fear learning by engaging in a disinhibitory microcircuit, and when GABA activity was blocked in the cortex, the fear response was markedly reduced (Letzkus et al., 2011). Indeed, neuromodulation plays a key role in learning (Suga and Ma, 2003), and interneurons are both targets of extrinsic modulators such as neurotransmitters, and are also known to modulate their own activity (Bacci et al., 2005). In the awake cortex, synaptic inhibition was found to dominate during cortical responses to visual stimulation, which functions to spatially restrict the spread of neuronal activity and allows for a 
more precise response to a localized visual stimulus (Haider et al., 2013). Overall, interneurons play a critical role in cortical circuitry and are essential for the proper functioning of the cerebral cortex.

Interneurons are a heterogenous population of cells (Cauli et al., 1997), and can be categorized into different classes based on criteria including morphology, expression of peptides, synaptic properties, the subcellular domains they target in postsynaptic cells, and action potential patterns (Kawaguchi and Kubota, 1997). There are three main classes of interneurons, which all uniquely contribute to cortical circuit function (Rudy et al., 2011). Parvalbumin(PV)- and somatostatin-expressing interneurons are born in the medial ganglionic eminence (MGE), whereas the caudal ganglionic eminence (CGE) gives rise to 5HT3aRexpressing interneurons, including reelin- and VIP-positive cells (Inan et al., 2012; Rossignol, 2011; Rudy et al., 2011). PV-positive interneurons are fast-spiking interneurons, and are the most abundant type in the cortex (Hu et al., 2014). PV cells are divided into basket cells which target the cell body and dendrites of excitatory neurons, and chandelier cells which target the axon initial segment of excitatory neurons (Kawaguchi and Kubota, 1997). Somatostatinexpressing interneurons are a diverse group of interneurons (Riedemann, 2019), one type of which is the defined Martinotti cell, which is calbindin-expressing and extends an axon to inhibit the distal apical dendrite of excitatory neurons in layer I (Ma et al., 2006). CGE-derived interneurons express $5 \mathrm{HT} 3 \mathrm{aR}$, a serotonin receptor, and can largely be divided into vasoactiveintestinal peptide (VIP)-expressing and VIP-lacking cells (Lee et al., 2010; Xu et al., 2010b). Overall, interneurons are incredibly diverse and contribute to the function of cortical circuitry in different ways, and elucidating the unique properties of each subtype will be critical to our understanding of cortical function. 


\section{Discovery of tangentially-migrating interneurons}

Interneurons were first described by Ramon y Cajal as short-axon cells that had a horizontal morphology (Ramon y Cajal, 1895). Later histological studies revealed that certain cells migrated in a direction that was tangential to radially migrating neurons in the cortex in rabbits (Stensaas, 1967), opossums (Morest, 1970), and mice (Shoukimas and Hinds, 1978). Lineage tracing studies using a retrovirus identified cells traveling perpendicular to radial glial fibers (Austin and Cepko, 1990; Walsh and Cepko, 1988). A similar observation of tangentiallyoriented cells in the neocortex was reported in a mouse model which labeled nearly half of all cells using X-inactivation mosaics (Tan and Breen, 1993). This tangential mode of migration was first observed in pioneering time-lapse imaging studies of ferret cortical slices, where Dillabeled neurons were observed migrating orthogonal to the radial direction (O'Rourke et al., 1992), however the origin of these tangentially-oriented cells remained unknown at the time.

By placing Dil and DiA crystals into the lateral ganglionic eminence (LGE), which is the nascent striatum located in the ventral telencephalon (Deacon et al., 1994), labelled cells from cultured slices were observed migrating tangentially in the preplate in the dorsal telencephalon (de Carlos et al., 1996). Another group also performed a similar experiment by using Dil to label LGE-derived cells in culture, however they also were able to show that these cells contained GABA, and hence showed that GABAergic interneurons migrated from the basal forebrain to the neocortex (Anderson et al., 1997). In this same study, brain slices were transected at the pallial-subpallial boundary (PSPB) on one side of the slice, and after being cultured, the transected sides had ten times fewer GABAergic neurons in the cortex, suggesting they had originated from below the PSPB (Anderson et al., 1997). In utero experiments revealed similar results, in which the PSPB was sectioned in one hemisphere in utero, then one day later in development, a buildup of GABAergic cells was located in the subpallium of the sectioned side of the brain (Tamamaki et al., 1997). These studies collectively suggested that interneurons were born in the subpallium and migrated tangentially across the PSPB to reach the cortex. 


\section{Generation of interneurons}

Cortical interneurons are born in the ganglionic eminences, which are so named because they will later give rise to the basal ganglia (Fentress et al., 1981), and are divided into the medial, lateral, and caudal ganglionic eminences (MGE, LGE, and CGE, respectively). Early studies first suggested that cortical interneurons were born in the LGE and MGE, when a Dil crystal was placed into the LGE or MGE of cortical slices, and Dil-labeled GABAergic cells were found in the cortex (Anderson et al., 1997; Lavdas et al., 1999). However, in a separate study, Dil was injected specifically into the MGE or LGE in cultured brain slices, and it was discovered that only cells from the MGE, and not the LGE, migrated into the cortex (Wichterle et al., 1999). Two separate studies in 2001 helped resolve this point of confusion. First, fatemapping of interneurons was performed by transplanting Bromodeoxyuridine- (BrdU) labeled MGE or LGE into unlabeled recipient slices, which revealed that the MGE is the primary source of tangentially migrating cells in the cortex during early development (Anderson et al., 2001). A second study used an ultrasound-guided in utero approach to transplant prelabeled MGE or LGE donor cells into a wild type embryo, and demonstrated in vivo that the MGE, and not the LGE, gives rise to cortical interneurons (Wichterle et al., 2001). Thus, the cortical interneurons that were observed following Dil placement in the LGE (Anderson et al., 1997) were actually MGE derived cells that had picked up the label while migrating through the LGE territory.

Additional evidence suggesting interneurons arise in the MGE came from studies of a Nkx2.1 knockout mouse. The transcription factor Nkx2.1 is expressed in the MGE, and in the Nkx2.1-/- model, interneurons were significantly reduced in the cortex (Sussel et al., 1999). It was later demonstrated that the CGE also gives rise to cortical interneurons through experiments using in vivo fate mapping approaches (Nery et al., 2002), which has since been confirmed in other studies (Butt et al., 2005; Lopez-Bendito et al., 2004). Overall, approximately $70 \%$ of interneurons arise from the MGE, $30 \%$ from the CGE, and a small fraction from the preoptic area (Gelman et al., 2009; Miyoshi et al., 2010). MGE-derived interneurons in the 
mouse are first generated around embryonic (E) day 9.5, and peak around E13.5, whereas CGE-derived interneurons are first generated around E12.5, and peak at E15.5 (Miyoshi et al., 2007; Miyoshi et al., 2010).

\section{Interneuron migration}

Interneurons migrate a long distance from their place of origin in the ganglionic eminences to reach their final targets in the cortex, and rely on both intra- and extra-cellular guidance molecules and signaling gradients to effectively navigate the developing forebrain. During their migration, interneurons dynamically extend and retract branches to sense and respond to cues in their environment, which steers them into the correct direction (Ang et al., 2003; Nadarajah et al., 2003). Interneurons then move forward through a process called nucleokinesis, or somal translocation (Bellion et al., 2005; Moya and Valdeolmillos, 2004). Additionally, proper positioning of intracellular organelles in interneurons, including the primary cilium, has also been shown to play a role in their guided migration (Baudoin et al., 2012; Higginbotham et al., 2012). Disruptions to the primary cilium in interneurons impairs the neuron's morphology, and as a result alters the synaptic connectivity in the cortex (Guo et al., 2017).

Interneurons are repelled from both the MGE and LGE through chemorepulsive cues to direct interneuron migration out of the ventral telencephalon and towards the cortex (Wichterle et al., 2003; Zhu et al., 1999). Ephrin-A5 is expressed in the ventricular zone of the ganglionic eminences, and serves as a chemorepulsive cue through the EphA4 receptor on interneurons (Rudolph et al., 2010; Zimmer et al., 2008). Another chemorepellent in the striatum is semaphorin, which repels interneurons expressing neuropilin receptors (Marin et al., 2001). In addition to being repelled from the ganglionic eminences, chemoattractive factors present in the cortex attract interneurons. For instance, Neuregulin-1 (Flames et al., 2004) in the cortex 
attracts interneurons expressing the receptor Erbb4 (Yau et al., 2003), and stromal-derived factor 1 (SDF1, also known as Cxcl12) attracts interneurons expressing Cxcr4 (Li et al., 2008; Lopez-Bendito et al., 2008). Interneurons reach the cortical rudiment by embryonic (E) day 12 , after which they continue their tangential migration into the neocortex in streams (Anderson et al., 2001; Lavdas et al., 1999).

Interneurons migrate tangentially in organized streams in the cortex, traveling through the marginal zone (MZ), subventricular zone (SVZ), and subplate streams (Wichterle et al., 2001). Interestingly, the origin of the cortical interneuron (MGE or CGE) does not determine which migratory stream the interneuron will choose (Miyoshi and Fishell, 2011), however the cortical environment can have a major impact on interneuron stream maintenance. One chemoattractant that interneurons utilize to stay in streams is $\mathrm{Cxcl12}$, which is expressed in the cortex in the meninges near the $\mathrm{MZ}$, and in progenitor cells in the SVZ region (Stumm et al., 2003; Tiveron et al., 2006). Interneurons sense Cxcl12 signaling through the expression of the receptors Cxcr4 and Cxcr7, and indeed, with genetic loss of either of these two receptors, cortical interneurons prematurely exit their streams and deposit into the cortical plate (Abe et al., 2014; Sanchez-Alcaniz et al., 2011; Wang et al., 2011). The timing of stream exit is a critical developmental process, because alterations at this stage can lead to lasting changes in the layering of inhibitory interneurons, which can directly affect the delicate balance between excitation and inhibition in the cortex (Lopez-Bendito et al., 2008).

\section{Layering with cortical excitatory neurons}

Interneurons travel in tangential streams into the cortex, switch to a radial mode of migration to enter the cortical plate, but then must transition out of their motile state in order to terminate in the appropriate location in the cortical wall. It has been shown that gammaaminobutyric acid (GABA; Cuzon et al., 2006; Inada et al., 2011) and glutamate (Manent et al., 
2006) expression in the cortical wall enhances the migration of interneurons in mouse embryos, acting through $\mathrm{GABA}_{\mathrm{A}}$ (Soria et al., 1999) and AMPA (Metin et al., 2000) receptors, respectively, located on migratory interneurons. GABA and glutamate initially enhance interneuron migration during embryogenesis by depolarizing the interneuron membrane to increase calcium transients inside the cell (Metin et al., 2000; Soria et al., 1999). However, during early postnatal development, GABA switches from a motile cue to a stop signal for cortical interneurons (Bortone and Polleux, 2009). KCC2, a potassium/chloride exchanger, is expressed on interneurons several days after reaching the cortex, and shifts the interneuron's response to GABA from depolarizing to hyperpolarizing (Bortone and Polleux, 2009). Additional evidence supported the notion that $\mathrm{KCC} 2$ upregulation is at least in part responsible for migration termination, when MGE-derived interneurons were found to upregulate $\mathrm{KCC} 2$ earlier than CGE-derived interneurons, and MGE-derived cells terminate their migration earlier than their CGE counterparts (Miyoshi and Fishell, 2011).

In addition to slowing and stopping their migration, cortical interneurons must decide where to deposit in the cortical wall, and several different factors including the location and time of their generation have been shown to play a role in this decision process. Interneurons generated from the MGE and CGE layer in two distinct patterns, with MGE-derived interneurons layering in an inside-out pattern, and CGE-derived interneurons contributing $75 \%$ of their population to upper layers, and $25 \%$ to lower layers, regardless of their birth dates (Miller, 1985; Miyoshi et al., 2010). Additionally, two different studies showed that interneurons born at the same embryonic time point, determined by viral injections, tended to cluster together in the cortex in vertical or horizontal columns (Brown et al., 2011; Ciceri et al., 2013). However, fatemapping studies determined that the final laminar organization in the cortex was dependent on the region of origin of the interneuron, and therefore the subtype, rather than by the date of birth (Miyoshi and Fishell, 2011). Interneurons born in the MGE and CGE at E12.5 primarily resided in complementary deep and superficial layers, respectively, even though they were generated at 
the same time (Miyoshi and Fishell, 2011). Additionally, interneurons born in either the MGE or CGE layered according to their place of birth, even when transplanted into the opposite ganglionic eminence, and were allowed to migrate into the cortex from the altered location (Butt et al., 2005; Nery et al., 2002).

The cortical environment into which inhibitory interneurons are migrating also likely plays a role in determining the final positioning of interneurons. Interneurons were shown to enter their final laminar position in the cortical wall well after their age-matched cortical excitatory neurons had reached their destinations (Pla et al., 2006). Heterochronic transplantations of MGE-derived interneurons into host mouse embryos additionally revealed that both early (E12.5) and late (E15.5) born interneurons can switch their laminar fates based on the age of the new cortical environment (Valcanis and Tan, 2003). Indeed, the organization and identity of cortical excitatory neurons in the developing cortical plate can directly impact the layering of inhibitory interneurons, as evidenced by disrupted interneuron distribution in mutants with excitatory neuron layering defects (Hevner et al., 2004; Lodato et al., 2011).

\section{Cortical circuitry diseases and development}

Disorders thought to arise from a common abnormality in brain development can be studied in tandem to try to gain a deeper understanding of underlying causes and pathologies. Several neurologic/neuropsychiatric disorders, including autism, epilepsy, and schizophrenia, have been suggested to arise from an imbalance between excitation and inhibition in the cerebral cortex (Doherty and Owen, 2014; Kato and Dobyns, 2005; Rossignol, 2011). Although the age at which clinical symptoms emerge is different for all of these diseases, the critical time frame shaping the course of the disease may arise very early in postnatal life, or even as early as embryogenesis. Disruptions in genes that control many different aspects of interneuron 
development, including genes that control the generation, migration, and function of interneurons, can contribute to the etiologies of these various disorders (Rossignol, 2011).

In Autism Spectrum Disorder (ASD), a very heterogeneous disorder in regards to presentation, one hypothesis of the common underlying mechanism is an imbalance between excitation and inhibition in the brain (Uzunova et al., 2016), which was first suggested as a model for the disease in 2003 (Rubenstein and Merzenich, 2003). In patients with ASD, levels of glutamic acid decarboxylase (GAD) 65 and 67, the enzymes the convert glutamate to GABA in the brain, were reduced in the cortex (Fatemi et al., 2002).

Mutations in the methyl-CpG binding protein 2 (MECP2) gene are known to cause ASD, along with other related disorders including Rett syndrome (Amir et al., 1999; Chahrour and Zoghbi, 2007). Rett syndrome is characterized by stereotyped behaviors, cognitive defects, and seizures, and in mouse models of the disease, cortical function was shown to be altered due to changes in the excitation/inhibition balance in the brain (Dani et al., 2005). One known target of the MECP2 gene is $D / x 5$, which regulates interneuron development (Horike et al., 2005). Intriguingly, it was shown that specific loss of MECP2 from GABAergic interneurons recapitulated behavioral features of Rett syndrome, and caused cortical hyperexcitability (Chao et al., 2010).

Schizophrenia is a disorder that presents in late adolescence, yet the pathological mechanisms underlying this disorder are thought to occur much earlier in brain development. Studies from postmortem tissue of patients with schizophrenia show altered levels of interneuron marker genes, demonstrating a different proportion of interneuron subtypes (Fung et al., 2014; Fung et al., 2010). Additionally, the interneurons most altered in schizophrenia originated primarily from the MGE, showing a developmental structure that may be altered in a disease showing symptoms twenty or more years later (Fung et al., 2014). Therefore, analyzing genes that affect the development of different populations of interneurons or their embryonic precursors can help provide insight into mechanistic causes of diseases such as schizophrenia. 
Epilepsy can manifest during childhood or adolescence, and its etiology may stem from disruptions to inhibitory circuit formation during development. One of the first studies linking developmental interneuron dysfunction to later epileptic behavior was performed by Powell and colleagues in Pat Levitt's laboratory. In a mouse model lacking urokinase-type plasminogen activator receptor (u-PAR), a cell membrane receptor, interneurons aberrantly migrated out of the ganglionic eminences, and as a result, there were reduced numbers of interneurons in the embryonic and postnatal cortex (Powell et al., 2001; Powell et al., 2003). In the adult animals, mice display higher anxiety-like behaviors, have spontaneous seizures, and are more susceptible to pharmacologically induced seizures (Powell et al., 2003). Interestingly, the parvalbumin subtype of interneurons was found to be predominately affected (Powell et al., 2003). While therapies for epilepsy have advanced, there are still treatment-resistant seizures that are debilitating. A recent treatment option being explored is the implantation of MGEderived interneuron precursors into seizure foci (Jaiswal et al., 2015). Studying the migration patterns of these and other interneuron subpopulations will enable the therapeutic strategies for previously untreatable disorders to become more effective.

Several other disorders with known interneuron migration deficits early in development leading to disorders later in life have been identified. 22q11 deletion syndrome, also known as DiGeorge syndrome, leads to a wide variety of clinical phenotypes including delayed neurodevelopmental function and associated behavioral challenges, and is also a significant risk factor for autism and schizophrenia (Niklasson et al., 2009). Mouse models of 22q11 deletion syndrome demonstrated altered interneuron migration during development, and later aberrant distribution of interneurons in the adult cortical wall (Meechan et al., 2009; Meechan et al., 2012b), which was accompanied by cognitive impairment due to changes in cortical circuitry (Meechan et al., 2015).

Another neurodevelopmental disorder, X-linked lissencephaly, is the first human disorder linked to a deficit in the tangential migration of interneurons. X-linked lissencephaly is a 
disorder associated with a smoother appearance of the brain, mental retardation, intractable epilepsy, and developmental delays, and has been shown to be caused by mutations in the aristaless-related, homeobox ( $A R X)$ gene (Kato et al., 2004; Stromme et al., 2002). Mice with mutations in $A R X$ had aberrant tangential migration of interneurons during development, and a subsequent loss and abnormal distribution of interneurons in the cortical plate (Kitamura et al., 2002). This was consistent with neuropathological findings of postmortem human brains at late gestation, in which a lack of interneurons in the cortex was identified (Bonneau et al., 2002). Since the excitation/inhibition imbalance in the brain was caused by tangential migration defects early in life, the new term "developmental interneuronopathy" was proposed to categorize epilepsies caused by early disruptions to interneurons (Kato and Dobyns, 2005), highlighting the importance of this cell population in neurodevelopmental diseases.

\section{Thalamocortical pathway}

\section{The role of the thalamus}

The thalamus is located in the diencephalon and can be divided into dozens of structurally and functionally distinct nuclei (Jones, 2007). Nuclei primarily in the dorsal region of the thalamus send projections to different cortical areas, relaying essential motor and sensory input from different areas of the body and the brain to the cortex (Jones, 2001). In addition to the traditionally-described role as a sensory-motor relay station, the thalamus also plays a critical role in allowing the cortex to communicate to other higher cortical areas through a cortico-thalamo-cortical pathway (Sherman and Guillery, 2011). Indeed, when thalamic activity was chemically inhibited, the trans-thalamic activation of other cortical areas was silenced, demonstrating a critical role of the thalamus in relaying information not only from the body to the cortex, but between cortical areas (Theyel et al., 2010). 
The topography of thalamocortical projections is highly organized, with rostromedial and caudolateral thalamic nuclei projecting to more rostral and caudal cortical areas, respectively (Caviness and Frost, 1980). Most thalamic nuclei project to a defined cortical region through topographical sorting that is established during embryonic development before any external sensory input is received (Catalano et al., 1996; Dawson and Killackey, 1985). In addition to the higher-level projection patterns by distinct thalamic nuclei, each nucleus shows a precise internal topographic organization, which transfers a topographical replica of the sensory surface to the neocortex (Woolsey and Van der Loos, 1970). For example, in the rodent somatosensory map, whiskers form a barreloid map in the ventroposterior nucleus of the thalamus, which is then transferred to a barrel map in the primary somatosensory cortex, in a pattern that emerges during early development (Killackey et al., 1990; Wu et al., 2011). Even by birth (postnatal day 0) in the mouse, the topography of thalamocortical axons in the deep cortical layers of the barrel cortex is highly precise (Agmon et al., 1995), and by postnatal day 3, a whisker pad-like pattern emerges in the cortex (Rice et al., 1985).

Any disruptions to this high level of topographic precision in early thalamocortical axon pathfinding can have long-term implications in thalamic connectivity as well as cortical organization and function (Anton-Bolanos et al., 2018). The sorting of thalamocortical axons in the ventral telencephalon is critical for the transfer of the whisker map to the neocortex, as disruptions to the transcription factor Ebf1 in the basal ganglia caused somatosensory thalamocortical axons to shift toward the visual cortex, and as a result, thalamic axons failed to form a topographic barrel map (Lokmane et al., 2013). Thalamocortical input is not only essential for proper mapping of sensory surfaces, but it has also been shown to directly influence the size and gene expression patterns of different cortical areas (Vue et al., 2013). Alterations in the connectivity between the thalamus and cortex have been implicated in various disorders, including those that arise from disruptions during development. 


\section{Thalamocortical connectivity and neurodevelopmental disorders}

Neurodevelopmental disorders associated with excitation/inhibition imbalances have also been associated with altered thalamocortical circuitry, including schizophrenia (Karlsgodt et al., 2008), epilepsy (Pulsipher et al., 2011), and autism (McFadden and Minshew, 2013). Schizophrenia is a neuropsychiatric disorder characterized by altered perceptions of reality and distorted thoughts that interfere with daily function, and is thought to arise from developmental alterations to neural connectivity (Canu et al., 2015; Pantelis et al., 2003). Anatomic analyses first revealed disruptions to white matter axonal tracts as well as changes in the size of the thalamus of patients with schizophrenia (Jones, 1997; Shenton et al., 2001). Technological advances have since allowed for more sophisticated approaches to understand the relationship between structure and function in the human brain. Resting state functional magnetic resonance imaging (fMRI) was used to map functional connectivity between the cortex and thalamus, and indeed, activity in specific cortical areas correlated with distinct regions in the thalamus (Zhang et al., 2008; Zhang et al., 2010). When compared to control subjects, patients with schizophrenia were found to have increased motor/somatosensory-thalamic and reduced prefrontal-thalamic connectivity (Woodward et al., 2012). This study demonstrated functional alterations in thalamocortical circuitry in schizophrenia, and revealed that separate thalamic circuits could be differentially affected in this disorder (Woodward et al., 2012).

Another neurodevelopmental disorder in which altered thalamocortical circuitry has been implicated is epilepsy, a disease characterized by repeated seizures due to abnormal excessive/synchronous electrical activity in the brain (Patel and Moshe, 2020). fMRI studies showed activation of the thalamus in patients with idiopathic generalized epilepsy, as well as in a patient having an absence seizure (Aghakhani et al., 2004; Salek-Haddadi et al., 2003). In patients with temporal lobe epilepsy, brain recordings revealed increased synchrony between the thalamus and temporal lobe during seizures, and the degree of synchrony was correlated with an early loss of consciousness (Guye et al., 2006). Based on the patterns of activation and 
deactivation in the thalamus and frontal/parietal regions in patients having seizures, it was postulated that the altered connections between the thalamus and cortex during seizures is also responsible for the reduced state of responsiveness in patients (Gotman et al., 2005).

Autism spectrum disorder (ASD) and attention-deficit hyperactivity disorder (ADHD) both manifest in early childhood, and disruptions in thalamocortical connectivity have been identified in both diseases. In patients with ASD, reduced functional connectivity to the thalamus was found during a face recognition task, which is a social cognition known to be impaired in affected individuals (Kleinhans et al., 2008). In a study combining both structural and functional imaging of patients with ADHD, white matter tracts were reduced in volume, and functional connectivity in the thalamus was reduced (Qiu et al., 2011). It will be critical to understand the relationship between structural alterations in connectivity and their resulting functional and behavioral consequences.

\section{The development of thalamocortical connections}

Thalamocortical axons must extend a lengthy distance over a long period of time during development in order to reach their intended targets in the cortex, and they do so through a highly orchestrated process. The dorsal thalamus is organized into different nuclei that are morphologically and functionally distinct, which begin to develop at E10.5 in the mouse brain (Angevine, 1970; Scholpp and Lumsden, 2010). Thalamocortical axons extend from the thalamus beginning at E11.5, traverse through the prethalamus, then cross the diencephalontelencephalon boundary (DTB) beginning at E12.5 (Braisted et al., 1999). In the ventral telencephalon, thalamocortical axons are guided by classical axon guidance molecules as well as intermediate targets including corridor cells positioned in the internal capsule region (LopezBendito et al., 2006; Molnar et al., 2012). At the pallial-subpallial boundary around E14.5, ascending thalamocortical and descending corticothalamic axons extend in such close proximity 
to one another, that they were thought to guide each other's extension in the so-called "handshake hypothesis" (Molnar and Blakemore, 1995). Once arriving to the cortex, thalamocortical axons accumulate in the subplate region of the developing cortical wall before synapsing onto their final targets in the cortical plate (Lund and Mustari, 1977; Shatz and Luskin, 1986).

\section{Thalamocortical axon repulsion from the hypothalamus}

After exiting the thalamus ventrally and traversing the prethalamus, thalamocortical axons must make a sharp lateral turn to exit the diencephalon and enter the telencephalon in route to the cortex. It was first discovered that thalamocortical axons did not enter the hypothalamus by visualizing early embryonic thalamocortical axons in fixed brain slices using Dil (Braisted et al., 1999). Additionally, axons from thalamic explants were repelled by the hypothalamus in vitro, however the guidance cue directing this repulsion was unknown (Braisted et al., 1999). Slit proteins were found to be responsible for the hypothalamic repulsion after thalamocortical axons in Slit2 and Slit1/Slit2 knockout mice aberrantly projected into the hypothalamus instead of crossing the diencephalon-telencephalon boundary (DTB) region (Bagri et al., 2002). In situ hybridization revealed high levels of Slit1 and Slit2 in the hypothalamus in the developing mouse embryo, along with Robo1 and Robo2 receptor expression in the dorsal thalamus (Bagri et al., 2002). The temporal expression of this receptor/ligand combination would allow for Slits to be the repellant for thalamocortical axons in the hypothalamus, along with the fact that Slit was found to be a midline repellent for Roboexpressing axons in Drosophila (Kidd et al., 1999) and in the developing vertebrate spinal cord (Brose et al., 1999; Long et al., 2004).

Slit2 was shown to be a repellant for thalamocortical axons in vitro when axons from thalamic explants turned away from Slit2-expressing COS cells in culture (Lopez-Bendito et al., 
2007). In this same study, Robo2 and Robo1/Robo2 knockout mice displayed similar axonal phenotypes to the Slit1/Slit2 knockout mouse, in which thalamocortical axons aberrantly invaded the hypothalamus (Lopez-Bendito et al., 2007). The repulsion of thalamic axons away from the hypothalamus in vitro was then confirmed in a rat model (Braisted et al., 2009), after being originally demonstrated in a mouse model (Braisted et al., 1999). Additionally, when Slit function is blocked in a hypothalamic explant, axons from thalamic explants grow towards the Slit-deficient hypothalamus, further suggesting Slit mediates the repulsion of thalamocortical axons away from the hypothalamus (Braisted et al., 2009). Moreover, in mouse mutants with loss of Gbx2 or Lhx2 from the thalamus, Robo1 and Robo2 expression was disrupted in thalamic axons, which caused thalamocortical axons to misroute into the hypothalamus (Chatterjee et al., 2012; Marcos-Mondejar et al., 2012).

\section{Thalamocortical axon crossing at the diencephalon-telencephalon boundary (DTB)}

Repulsion from the hypothalamus via the secreted protein Slit is one means by which thalamocortical axons are directed towards the telencephalon (Bagri et al., 2002; Braisted et al., 2009; Lopez-Bendito et al., 2007). In addition to diffusible chemorepellents from the hypothalamus, a population of cells known as "guidepost cells" have also been suggested to be important in guiding thalamocortical axons across the critical diencephalon-telencephalon boundary. Guidepost cells have their cell bodies located in the thalamocortical axon pathway, and extend an axon to the dorsal thalamus. While no specific marker exists for these cells, they can be identified by retrograde labeling of axons and cell bodies when a Dil crystal is placed into the developing dorsal thalamus (Godement et al., 1987). The idea of guidepost cells in thalamocortical connectivity was first formulated when cells located in the thalamic reticular nucleus were shown to project to the dorsal thalamus in a rat embryo (Mitrofanis and Guillery, 
1993) and later a ferret (Mitrofanis, 1994). Guidepost cells located in the internal capsule region were first discovered in a hamster (Metin and Godement, 1996), and later, in rat embryos, when retrogradely labeled cells with axons that extended into the dorsal thalamus were detected in the internal capsule near the coursing thalamocortical axons (Molnar et al., 1998). In the mouse model, guidepost cells were present in the internal capsule at E12.5, which is when the first thalamic axons arrive at the DTB, and have not yet entered the internal capsule (Braisted et al., 1999). More recent analyses showed these retrogradely labeled guidepost cells are Islet1positive, and many come from the D/x5/6 lineage of cells in the ventral telencephalon (Feng et al., 2016). While the ultimate fate of these cells remains unknown, embryonic analyses examining the presence of retrogradely labeled cells in the rat brain demonstrated that their number diminish over the course of development and their distribution in the telencephalon becomes more disperse (Molnar and Cordery, 1999).

In several different mouse models where thalamocortical axons are unable to cross the DTB, the guidepost cells have been reported as reduced or missing. In the Mash1 mutant, guidepost cells were completely absent from the ventral telencephalon, and no thalamocortical axons had crossed the DTB (Tuttle et al., 1999). Guidepost cells were still present in the Pax6, Emx2, and Sema6A mutants, however the cells were displaced and more spread out in the telencephalon (Jones et al., 2002; Lopez-Bendito et al., 2002; Mitsogiannis et al., 2017). Reductions in the numbers of guidepost cells were observed in Lhx2-/-, OL-protocadherin-/-, and DIx5/6-Cre;Celsr3 ${ }^{f / f l}$ mice (Feng et al., 2016; Lakhina et al., 2007; Uemura et al., 2007). Since no molecular marker currently exists to label these cells exclusively, it is unknown in mutants with missing/reduced number of guidepost cells whether the cells are no longer present, or whether they are present and are unable to extend an axon to the dorsal thalamus. 


\section{Navigation of thalamocortical axons through the telencephalic corridor}

After crossing the DTB, thalamocortical axons must extend through the ventral telencephalon before reaching the cortex. A population of cells called the "corridor" cells guide thalamocortical axons in the ventral telencephalon (Lopez-Bendito et al., 2006). Corridor cells originate in the lateral ganglionic eminence, and migrate both medially and caudally beginning at E11.5 to form a permissive territory through the $N k \times 2.1$-postiive medial ganglionic eminence (MGE) and globus pallidus (GP), which are normally not permissive to thalamocortical axons (Lopez-Bendito et al., 2006). Corridor cells express a membrane bound isoform of the Neuregulin-1 protein, CRD-NRG1, which attracts thalamocortical axons expressing the receptor ErbB4 (Lopez-Bendito et al., 2006). In addition to providing guidance through the ventral telencephalon, the caudal migration of corridor cells to the DTB is important in the early guidance of thalamocortical axons as they first enter from the diencephalon. In mouse embryos, the corridor region extends caudally and thalamocortical axons cross the DTB, however in chick embryos, the corridor extends medially but not caudally, and thalamocortical axons do not cross the DTB region (Bielle et al., 2011a). This study also demonstrated that corridor cells express Robo1 and Robo2 receptors, and they migrate caudally due to Slit2medaited repulsion from the MGE and preoptic area (Bielle et al., 2011a).

Several different mouse models with thalamocortical axon guidance defects at the DTB have disruptions to the corridor region. In the Slit2-/- mice, Ebf1-positive corridor cells fail to extend caudally to the DTB region (Bielle et al., 2011a). In Frizzled3-/- mice, Islet-1 positive cells expand into the GP region, and the Nkx2.1-postiive domain of the GP occupies a larger area (Morello et al., 2015). A similar invasion of Islet1-positive cells into the GP occurred in the OL-protocadherin-/- mouse, which was accompanied by a failure of Islet1-positive cells to extend caudally (Uemura et al., 2007). Furthermore, Islet1-positive cells were more broadly distributed in the ventral telencephalon of the Pax6-/- mouse (Simpson et al., 2009). It is still unknown whether the corridor cells are responsible for thalamocortical axon guidance strictly in 
the ventral telencephalon, or if they can also assist in the thalamocortical axon extension across the DTB in vivo. In slice culture in vitro assays where the DTB remained intact, a wild type corridor graft was able to partially rescue the axon misrouting phenotype in the Slit2-/- mouse, suggesting the corridor may play a role in DTB crossing (Bielle et al., 2011a).

\section{Sorting of thalamocortical axons in the ventral telencephalon}

Once in the ventral telencephalon, thalamocortical axons are sorted by gradients of guidance molecules expressed along the rostrocaudal axis. Early studies demonstrated that the ventral telencephalon was attractive for thalamocortical axons in vitro when axons from thalamic explants grew towards telencephalic explants in a collagen assay (Braisted et al., 1999). One of the attractive factors was identified as Netrin-1, when axons from thalamic explants grew towards Netrin-1-expressing cells (Braisted et al., 2000). However, Netrin-1-blocking antibodies only slightly decreased the biased growth of thalamic axons to telencephalic explants, suggesting other chemoattractive factors may also be expressed by the telencephalon (Braisted et al., 2000). Additionally, in Netrin1-/- mice, although the crossing of axons at the DTB remained unimpaired, thalamocortical axons were disorganized once in the internal capsule (Braisted et al., 2000). This study, combined with Netrin-1 expression in the ventral telencephalon at the time thalamocortical axons are first traversing that region (Metin et al., 1997; Serafini et al., 1996), implicate Netrin as a key signaling molecule in early thalamocortical axon pathfinding. Netrin-1 was found to be expressed in a high-rostral to low-caudal gradient in the ventral telencephalon (Powell et al., 2008). Netrin-1 is a chemoattractant for rostral thalamic axons which express high levels of the Dcc receptor that mediate Netrin-1's attractive effects, and is a chemorepellent for caudal thalamic axons which express high levels of the Unc5A/C receptors that mediate the repulsive effects of Netrin-1 (Powell et al., 2008). 
Two other classes of axon guidance molecules, semaphorins and ephrins, were identified as key regulators of the topographic sorting of thalamocortical axons in the ventral telencephalon. Semaphorins3A/3F and EphrinA5 are expressed in high-caudal to low-rostral gradients in the telencephalon, and act through Sema6A and EphA3/4/7 receptors on thalamic axons, respectively, to appropriately sort thalamocortical axons as they are coursing through the ventral telencephalon (Dufour et al., 2003; Leighton et al., 2001). Gradients of EphrinA5 have been shown to prevent the caudal growth of rostral thalamic axons, which express high levels of EphA3/4/7 receptors, and gradients of Semaphorin3A/4F have been shown to prevent the caudal growth of intermediate thalamocortical axons (Dufour et al., 2003; Little et al., 2009; Wright et al., 2007). More recent work has demonstrated that gradients of Slit and Netrin act together to guide thalamocortical axons in the ventral telencephalon (Bielle et al., 2011b; LeyvaDiaz et al., 2014). FLRT3 was identified as a novel co-receptor for Robo1, and in the presence of Slit1 in the telencephalon, both Robo1 and FLRT3 receptors are required on thalamic axons in order for the axons to be attracted to the Netrin-1 gradients (Leyva-Diaz et al., 2014).

\section{Longitudinal axon pathways that cross the DTB in early development}

Thalamocortical axons first cross the DTB at E12.5 in the mouse, aided in part by Slitmediated repulsion from the hypothalamus, chemoattractants from the ventral forebrain, guidepost neurons and their axonal projections to the thalamus, and corridor cells in the ventral telencephalon. Besides thalamocortical axons, several other longitudinal axon pathways must cross the DTB during a similar developmental time window in route to their final targets. The two longitudinal axon pathways that reciprocally connect the striatum to the substantia nigra (striatonigral) and the substantia nigra to the striatum (nigrostriatal) cross the DTB at around the same time as thalamocortical axons (Garcia-Pena et al., 2014; Morello et al., 2015). 
Disruptions to both of these pathways have been identified in mouse mutants with aberrant thalamocortical connectivity.

OL-protocadherin is highly expressed in developing striatal cells located in the ventral telencephalon (Hirano et al., 1999), and in mouse knockout models, striatal axons fail to cross the DTB in vivo and are unable to properly extend in vitro (Uemura et al., 2007). In this same

model, both thalamocortical and nigrostriatal axons are misrouted into the hypothalamus instead of crossing the DTB (Uemura et al., 2007). Frizzled3 is a Wnt receptor, and has been shown to be critical for the extension of thalamocortical axons across the DTB region (Hua et al., 2014; Wang et al., 2002; Wang et al., 2006). In the Frizzled3-/- mouse, both striatonigral and nigrostriatal axons fail to properly extend, and as result do not cross the DTB region (Fenstermaker et al., 2010; Hua et al., 2014; Morello et al., 2015). Finally, in Slit1/2 double knockout mice, nigrostriatal axons are similarly misrouted into the hypothalamus (Bagri et al., 2002). Much remains to be determined on how and if these different axon pathways rely on each other for guidance, and how coordinated orchestration of axon pathways, corridor and guidepost cells, and signaling gradients work together during development to form a highly complex and organized brain.

\section{The c-Jun N-terminal Kinase (JNK) Signaling Pathway}

\section{JNK Signaling Cascade}

Intracellular signaling pathways are critical for the integration of extracellular cues that cells must respond to in order to interact with their environment. The c-Jun N-terminal Kinase (JNK) signaling pathway is a member of the mitogen-activated protein kinase (MAPK) signaling pathway, and is controlled by two upstream MAPK kinases, MKK4 and MKK7, which are activated by various MAPKKKs through phosphorylation (Weston and Davis, 2007; Yamasaki et al., 2012). As the terminal kinase in the signaling cascade, JNKs phosphorylate a wide variety 
of downstream substrates to regulate cellular behavior (Manning and Davis, 2003). JNK was initially discovered after being purified from rat liver 30 years ago (Kyriakis and Avruch, 1990), and has subsequently been shown to regulate normal processes of cell proliferation, protein degradation, cell movement, and apoptosis (Bogoyevitch and Kobe, 2006).

The JNKs are encoded by the three genes Jnk1, Jnk2, and Jnk3, also known as Mapk8, Mapk9, and Mapk10, respectively, and are alternatively spliced into ten different isoforms (Gupta et al., 1996; Kyriakis et al., 1994). Jnk1 and Jnk2 are ubiquitously expressed throughout the body, but Jnk3 is found primarily in the brain, and in smaller amounts in the cardiac smooth muscle and testes (Kuan et al., 1999; Martin et al., 1996). All three JNKs are expressed in both the developing and adult brain (Brecht et al., 2005; Kuan et al., 1999). Interestingly, JNK has been shown to be differentially regulated in neurons compared to non-neuronal cells and tissue. In non-neuronal cells, JNK is activated in response to stress (Davis, 2000); however, in neuronal cells, JNK1 is constitutively active, and JNK2 and JNK3 are stress-responsive (Coffey et al., 2000; Coffey et al., 2002). In non-neuronal cells, JNK has been implicated in the induction of autophagy (Ogata et al., 2006; Wei et al., 2008; Yu et al., 2004); however, in neuronal cells, JNK suppresses autophagy (Xu et al., 2011). These differences between neuronal and non-neuronal cells suggests JNK may play a unique role in the nervous system.

\section{The role of JNK in neuronal migration}

JNK has been shown to play a role in the migration of many different cell types, including fibroblasts, tumor endothelial cells, several cancer cell lines, and smooth muscle cells (Huang et al., 2003; Kavurma and Khachigian, 2003; Malchinkhuu et al., 2005). However, JNK's role in neuronal migration was first discovered in 2003 , when both a dominant-negative form for JNK (Derijard et al., 1994) and the JNK inhibitor SP600125 (Bennett et al., 2001) suppressed the migration of excitatory neurons in the developing cortical wall (Kawauchi et al., 2003). 
Additionally, studies using either SP600125 or a short hairpin RNA knockdown of Jnk1 demonstrated that JNK signaling was required for proper nuclear movements and leading process branching in migrating cortical excitatory neurons (Nishimura et al., 2010; Nishimura et al., 2014).

Several studies have shown disruptions to radial migration of neurons when upstream activators of JNK signaling are altered. Mitogen-activated protein kinase upstream protein kinase (MUK, also known as dual leucine zipper-bearing kinase- DLK), is a MAPKKK that activates JNK (Hirai et al., 1996), and when DLK is overexpressed in neural precursor cells, the migration of excitatory neurons is arrested in the subventricular zone of the cortical wall (Hirai et al., 2002). Additionally, in DLK-/- mice, as well as in cortical slices treated with SP600125, the acceleration of excitatory neurons in the subplate region of the cortex was delayed (Hirai et al., 2006). Excitatory neuron migration was also shown to be delayed when the two direct upstream activators of JNK signaling, MKK4 and MKK7, were genetically removed from neuronal cells in mice using the Nestin-Cre driver line (Wang et al., 2007; Yamasaki et al., 2011).

The effects of removing different JNK genes, as well as using genetic models to eliminate JNK function, have been utilized more recently. Excitatory cortical neurons in Jnk1 knockout mice displayed an increased speed of migration in early phases of excitatory neuron migration (Westerlund et al., 2011). Using short hairpin RNAs to selectively target Jnk2 or Jnk3 through in utero electroporation showed decreased migration of excitatory neurons with the knockdown of Jnk2, and no change in the migration with the knockdown of Jnk3 (Zhang et al., 2016). Our lab was the first to show a requirement for JNK signaling in the migration of cortical interneurons. In both pharmacological and genetic approaches, cortical interneurons were delayed in their entry into the cortex, prematurely dispersed from migratory streams, and inappropriately allocated in the early postnatal cortex (Myers et al., 2020; Myers et al., 2014). Additionally, JNK signaling is required for the proper dynamic behaviors of migrating 
interneurons, including leading process branching and nucleokinesis (Smith et al., 2020). JNK signaling therefore plays a role in the migration of both cortical excitatory neurons and inhibitory interneurons.

\section{JNK's role in axon pathfinding}

JNK signaling has also been shown to play a role in axon growth, regeneration, and pathfinding. Before JNK's specific role in axon development was determined, it was demonstrated that JNK function was necessary for neurite architecture and dendritic length (Bjorkblom et al., 2005; Coffey et al., 2000). JNK was first demonstrated to play a role in neuronal polarity when the active phosphorylated form of JNK was found to be highly enriched in the axon on neurons, and inhibition of JNK signaling in neurons in vitro prevented axonogenesis (Oliva et al., 2006; Tararuk et al., 2006). Interestingly, JNK has also been shown through multiple different studies to play an essential role in axon regeneration (Arthur-Farraj et al., 2012; Raivich et al., 2004), and recently, through a phosphoproteomics analysis, JNK was found to be activated in growth cones specifically in growing and regenerating axons (Kawasaki et al., 2018).

Studies in mouse models provided evidence for JNK's role in axons in vivo, through genetic knockouts of JNK itself, upstream activators of JNK signaling, or JNK binding proteins. Jnk2 knockout mice had normal development of the anterior commissure, however in Jnk1 knockout mice, the anterior commissure developed normally up until postnatal $(P)$ day 6 , but was completely lost by P12 (Chang et al., 2003). In mice with loss of DLK, the anterior commissure, corpus callosum, and other cortical tracts were disrupted (Hirai et al., 2006). Another mouse model that displayed severely disrupted axon tracts involved the conditional deletion of MKK7, an upstream activator of JNK signaling (Asaoka and Nishina, 2010; Davis, 2000). When MKK7 was removed from neural stem cells and postmitotic neurons using the 
Nestin-Cre driver line, knockout mice had enlarged ventricles, and reduced size of the striatum, internal capsule, anterior commissure, and corpus callosum (Yamasaki et al., 2011).

Interestingly, these severe axon phenotypes were not observed when MKK4, the other main upstream activator of JNK signaling, was removed from the same lineage (Wang et al., 2007). Finally, in mice deficient in JIP3 (JNK interacting protein 3), which can form a scaffold with JNK (Ito et al., 1999; Whitmarsh et al., 1998), both the corpus callosum and anterior commissure failed to cross the midline (Cho et al., 2011; Ha et al., 2005; Kelkar et al., 2003). Collectively, these studies suggest that JNK plays an important role in the development of major axon pathways in the developing forebrain.

\section{JNK and disease}

JNK has been shown to play a role in many different types of neurologic diseases, ranging from neurodevelopmental to neurodegenerative disorders. Postmortem brains from Alzheimer's and Parkinson's patients were found to have increased JNK activity (Ferrer et al., 2001; Ferrer et al., 2003), and JNK was actually found to localize to pathological brain regions containing plaques and tangles (Pei et al., 2001; Zhu et al., 2001). Through animal models, JNK has even been shown to increase amyloid-Beta plaque load and hyperphosphorylate tau in Alzheimer's brains (Mazzitelli et al., 2011; Reynolds et al., 1997).

Increasing evidence has been found for JNK's role in neuropsychiatric disorders, and patients with gene anomalies in the JNK pathway have presented with a variety of diseases. The first genetic link between JNK and schizophrenia was discovered in a patient with a mutation in the MAPKK7 gene, which is an upstream activator of JNK signaling (Winchester et al., 2012). Patients with autism have been found to have alterations in the TAOK2 gene, an upstream activator of JNK signaling (de Anda et al., 2012; Weiss et al., 2008), as well as mutations in GSTP1, a JNK regulatory protein (Williams et al., 2007). Mutations in the 
IL1RAPL1 gene have been associated with mental retardation and autism (Carrie et al., 1999; Piton et al., 2008), and mouse models have shown that IL1RAPL1 signals through JNK (Pavlowsky et al., 2010a; Pavlowsky et al., 2010b).

Widescale genetic analyses have associated JNK signaling with neurodevelopmental disorders. An array-comparative genomic hybridization was performed on patients with structural brain malformations, many of whom had Intellectual Disability, and gene ontology search revealed that genes in the JNK cascade are likely to play a significant role in the etiology of brain malformations (Kariminejad et al., 2011). Additionally, a separate study analyzed the gene expression profile of autism-susceptibility genes in the developing human brain, and found JNK to be a major signaling pathway central to autism spectrum disorder networks (Ziats and Rennert, 2011).

JNK3 specifically has been implicated in human disorders including epilepsy and cognitive dysfunction (Kunde et al., 2013; Shoichet et al., 2006). A case study revealed a truncated JNK3 protein in a patient with epilepsy and a learning disability, in which chromosome mapping showed that Jnk3 was disrupted in a de novo balanced translocation (Shoichet et al., 2006). A second unrelated patient with intellectual disability had a similar mutation in Jnk3, and in both patients, the mutations resulted in truncated proteins that were unable to phosphorylate the JNK target c-Jun in vitro (Kunde et al., 2013).

JNK knockout mouse models have provided additional insight into JNK's role in neurological disorders. In Jnk3 knockout mice, animals were found to be resistant to kainateinduced seizures, due to the neuroprotection offered by removal of JNK's ability to elicit neuronal death in the hippocampus (Yang et al., 1997). In Jnk1 knockout mice, animals displayed reduced depressive and anxiety-like behaviors (Mohammad et al., 2018). The precise role that JNK plays in all of these disorders still remains to be unraveled, but both patient and animal studies show evidence that disruption of the JNK signaling pathway can contribute to the etiology of these disorders. 


\section{JNK knockout animal models}

Prior studies investigating the loss of JNK function have utilized both in vivo mouse models as well as pharmacological inhibition of JNK signaling in order to gain insight into the role of JNK in neural development. Genetic approaches have largely examined the loss of only one or two JNK genes (Coffey, 2014; Zdrojewska and Coffey, 2014), and in one case all three from cerebellar Purkinje cells (Xu et al., 2011), which limits our understanding of JNK function as a whole, since JNK genes can partially compensate for one another. Indeed, JNK2 deficiency through a chemical genetic approach was found to cause a compensatory increase in JNK1 function (Jaeschke et al., 2006), and in Jnk2-/- mice, JNK1 levels are increased, and in Jnk3-/- mice, JNK1 levels were found to be increased in brain tissue (Brecht et al., 2005; Chen et al., 2005).

Generation of a mouse model with loss of all three JNK genes requires a conditional approach, since the constitutive deletion of just Jnk1 and Jnk2 is embryonic lethal in mice (Kuan et al., 1999; Sabapathy et al., 1999). Isoform-specific functions of JNK in neurons have been demonstrated in prior studies (Brecht et al., 2005), however removal of all JNK function is required to reveal roles for JNK in autophagy (Xu et al., 2011) and neuronal death (Bjorkblom et al., 2008). Indeed, the most promising neuroprotective effects observed in vivo from chemical inhibitors of JNK do not distinguish between JNK isoforms (Graczyk, 2013). Therefore, studies examining the loss of all three JNK genes will be critical to understand JNK's role in biological processes.

Studies examining the requirement for JNK in neuron migration and axon elongation have either explored the loss of one or two JNK genes in vivo, or used in vitro approaches of pharmacological inhibition. Prior to this thesis work, JNK's roles in interneuron stream maintenance, laminar allocation, and thalamocortical axon pathfinding were all unknown. In this dissertation, the requirements for JNK function in forebrain development are explored in vivo in a novel conditional triple knockout (cTKO) mouse model, where Jnk1 is conditionally eliminated 
from DIx5/6-lineage cells in a Jnk2;Jnk3 knockout background. This work demonstrates critical roles for JNK function in cortical inhibitory interneuron migration and allocation, and in early thalamocortical axon pathfinding. 


\title{
Chapter 2: JNK signaling is required for proper tangential migration and laminar allocation of cortical interneurons
}

\author{
Abigail K Myers ${ }^{1,2,4 \# *}$, Jessica G Cunningham ${ }^{1,2,4 \#}$, Skye E Smith ${ }^{1,3,4}$, John P Snow ${ }^{4}$, Catherine A \\ Smoot ${ }^{1,2,4}$, and Eric S Tucker ${ }^{1,4}$ \\ 1Department of Neuroscience, ${ }^{2}$ Neuroscience Graduate Program, ${ }^{3}$ Biochemistry Graduate \\ Program, ${ }^{4}$ Rockefeller Neuroscience Institute, West Virginia University School of Medicine, \\ Morgantown, WV 26506 \\ * Present address: Department of Pathology, Brigham and Women's Hospital, Harvard Medical \\ School, Boston, MA 02115 \\ \# These authors contributed equally to this work
}

Correspondence: etucker@hsc.wvu.edu

Published in Development, January $17^{\text {th }}, 2020$ https://dev.biologists.org/content/147/2/dev180646 


\begin{abstract}
The precise migration of cortical interneurons is essential for the formation and function of cortical circuits, and disruptions to this key developmental process are implicated in the etiology of complex neurodevelopmental disorders, including schizophrenia, autism, and epilepsy. We recently identified the c-Jun N-terminal kinase (JNK) pathway as an important mediator of cortical interneuron migration, regulating the proper timing of interneuron arrival into the cortical rudiment. In the current study, we demonstrate a vital role for JNK signaling at later stages of corticogenesis, when interneurons transition from tangential to radial modes of migration. Pharmacological inhibition of JNK signaling in ex vivo slice cultures caused cortical interneurons to rapidly depart from migratory streams and prematurely enter the cortical plate. Similarly, genetic loss of JNK function led to precocious stream departure ex vivo, and stream disruption, morphological changes, and abnormal allocation of cortical interneurons in vivo. These data suggest that JNK signaling facilitates the tangential migration and laminar deposition of cortical interneurons, and further implicates the JNK pathway as an important regulator of cortical development.
\end{abstract}




\section{Introduction}

Guided migration of cortical interneurons is mediated by the precise spatial-temporal integration of extracellular cues with intracellular signals. Failure to detect, transduce, or respond to these cues can alter the abundance, distribution, and connectivity of interneurons in the cerebral cortex, which may underlie severe neurological disorders like schizophrenia, epilepsy, and autism (Di Cristo, 2007; Kato and Dobyns, 2005; Marin, 2012).

Upon generation in the medial and caudal ganglionic eminences of the ventral forebrain, cortical interneurons migrate long distances to reach and infiltrate the cortical rudiment (Miyoshi et al., 2010; Nery et al., 2002; Xu et al., 2004). Within the developing cerebral cortex, interneurons predominantly travel in tangentially-oriented streams located in the marginal zone (MZ), subventricular zone (SVZ), and to a lesser extent, the subplate. As cortical development proceeds, interneurons gradually transition from tangential to radial modes of migration in order to leave their streams, layer in the cortical plate, and form synaptic connections (Miyoshi and Fishell, 2011). The mechanism by which cortical interneurons mediate this tangential to radial switch appears to be multifactorial, and has been attributed to several molecular pathways, including C-X-C motif chemokine ligand 12 ( $\mathrm{Cxcl} 12)$ and its receptors $\mathrm{Cxcr} 4 / \mathrm{Cxcr} 7$ (Li et al., 2008; Lopez-Bendito et al., 2008; Sanchez-Alcaniz et al., 2011; Wang et al., 2011), Connexin 43 (Elias et al., 2010), Sonic hedgehog (Baudoin et al., 2012), and Neuregulin 3 and its receptor ErbB4 (Bartolini et al., 2017). These pathways likely converge on cytoskeletal modulators, including Doublecortin and p27 kip1, which have been shown to influence the dynamic behavior of migrating cortical interneurons at key choice points in their trajectories (Friocourt et al., 2007; Godin et al., 2012; Kappeler et al., 2006; Lysko et al., 2014). To date, the intracellular signaling pathways that regulate the tangential progression of cortical interneurons in migratory streams and the timing of migratory stream exit are largely unknown.

The c-Jun N-terminal kinases (JNKs) comprise a subgroup of evolutionarily conserved mitogen-activated protein kinases. Three genes, Jnk1 (Mapk8), Jnk2 (Mapk9), and Jnk3 
(Mapk10), encode JNK proteins that function to transduce stressful extracellular stimuli, as well as enable normal physiological processes, including cell proliferation, apoptosis, differentiation, and migration (Davis, 2000). JNKs are implicated in multiple aspects of brain development, including neuronal migration in the cerebral cortex (Coffey, 2014). We previously found that JNK signaling controls the timing of interneuron entry into the cerebral cortex and the initial formation of tangential streams of migratory cells (Myers et al., 2014), however, the role that JNK plays in later stages of interneuron migration, including the tangential to radial switch, is unknown.

In the current study, we use pharmacological inhibition and genetic ablation of JNK function to investigate the role that JNK signaling plays in the tangential progression of cortical interneurons in migratory streams and the allocation of interneurons in the developing cortical wall. In live-imaging experiments from both pharmacologically treated and genetically manipulated ex vivo brain slices, cortical interneurons abandoned their tangential orientations, vacated streams, and prematurely entered the cortical plate. Genetic loss of JNK function in vivo resulted in delayed entry of interneurons into the cortex at E13.5, morphological abnormalities and early stream departure of interneurons at E15.5, and incorrect positioning of calbindin-positive and calbindin-negative interneurons in the postnatal day 0 cortical wall. Our findings suggest that JNK signaling is required for the maintenance of migratory streams and that inhibition of JNK may facilitate the switch from tangential to radial modes of migration in order to promote the infiltration of cortical interneurons into the nascent cortical plate. 


\section{Results}

\section{Loss of JNK signaling alters the radial distribution and migratory orientation of}

\section{cortical interneurons}

To determine if JNK signaling is required to maintain preformed streams of migratory cortical interneurons, we cultured live-vibratome sections from E14.5 D/x5/6-Cre-IRES-EGFP (DIx5/6-CIE) mouse brains in either control or JNK-inhibited conditions for 12 hours (Fig. 2.1A). Pharmacological inhibition of JNK signaling was attained using a pan-JNK inhibitor, SP600125 (Bennett et al., 2001), at concentrations previously shown to cause a delay in the entry of interneurons into the cerebral cortex (Myers et al., 2014). In control slices, interneurons travel tangentially in robust, organized streams within the marginal zone (MZ) and subventricular zone (SVZ), which were preserved at both the entry zone (lateral location) and the leading edge (medial location) of cortical interneuron migration (Fig. 2.1B-D). In JNK-inhibited slices, interneurons disbanded from migratory streams and entered the cortical wall after treatment with $20 \mu \mathrm{M}$ (Fig. 2.1E-G) and $40 \mu \mathrm{M}$ SP600125 (Fig. 2.1H-J). Disruption to the MZ and SVZ streams in JNK-inhibited slices occurred throughout the entire cortex, however significant alterations in cortical interneuron radial distribution were only present at the leading edge (Fig. 2.1L-M). Post-hoc analyses showed statistically significant decreases of cortical interneurons positioned in cortical bins roughly corresponding to the MZ, intermediate zone (IZ), and SVZ, as well as increases of cortical interneurons in the ventricular zone (VZ).

Since radial distribution analysis indicated that interneurons from JNK-inhibited slices were displaced from their migratory streams, we asked whether their direction of migration was altered. Distributions in the orientation of leading processes were significantly different between control and $20 \mu \mathrm{M} \mathrm{SP} 600125$, and between control and $40 \mu \mathrm{M}$ SP600125 conditions (Fig. 2.1K). In control conditions, $49.5 \%$ (99/200) of interneurons were tangentially oriented, whereas only $32.0 \%(57 / 178)$ of interneurons in the $20 \mu \mathrm{M} \mathrm{SP} 600125$ condition, and $32.0 \%(82 / 256)$ of 
interneurons in the $40 \mu \mathrm{M}$ SP600125 condition, maintained a tangential orientation. Collectively, these data suggest that JNK inhibition alters the migratory trajectory of cortical interneurons and leads to their departure from migratory streams.

\section{JNK inhibition disrupts migratory properties of cortical interneurons and causes premature departure from the SVZ stream}

Since fixed analyses indicated that JNK inhibition led to migratory stream departure, we performed live-imaging experiments on E14.5 DIx5/6-CIE brain slices to determine whether JNK inhibition altered the dynamic behavior of migratory cortical interneurons. Unlike in control slices, where interneurons mainly traveled tangentially in migratory streams (Fig. 2.2A-E,K; Supplemental Movie 2.1), cortical interneurons dramatically evacuated the SVZ stream in slices treated with $20 \mu \mathrm{M}$ SP600125 (Fig. 2.2F-J,L; Supplemental Movie 2.2). Breakdown of the SVZ stream began within a few hours of SP600125 treatment (Fig. 2.2G), and by 12 hours, interneurons had often vacated the SVZ stream (Fig. 2.2J). In control slices, $74.3 \%(107 / 144)$ of interneurons remained in the SVZ stream, whereas in SP600125-treated slices, only 29.9\% $(43 / 144)$ of the tracked cortical interneurons remained. This change in migratory behavior was accompanied by significant decreases in maximum, mean, minimum, and standard deviation of migratory speeds (values=mean \pm s.e.m.; control: $\max =146.5 \pm 3.28$, mean $=67.6 \pm 2.16$, $\min =10.3 \pm 0.67, S D=37.3 \pm 0.98 \mu \mathrm{m} /$ hour; $S P 600125: \max =103.0 \pm 4.80$, mean $=41.1 \pm 3.25$, $\min =2.88 \pm 0.53, S D=24.6 \pm 0.99 \mu \mathrm{m} /$ hour; Fig. $2.2 \mathrm{M}$ ), and hence an overall decrease in distance traveled (data not shown). Speed variability, which is the ratio of track standard deviation to track mean speed, was also significantly increased in JNK-inhibited conditions (control: 0.622 \pm 0.020 ; SP600125: 0.727 \pm 0.021 ; Fig. $2.2 \mathrm{~N}$ ). Compared to controls, migratory tracks of cortical interneurons in SP600125-treated slices were shorter in displacement over a 2-hour period of time (control: 111.1 $\pm 4.76 \mu \mathrm{m}$; SP600125: 51.9 $\pm 2.57 \mu \mathrm{m}$; Fig. $2.2 \mathrm{O}$ ), and had a more 


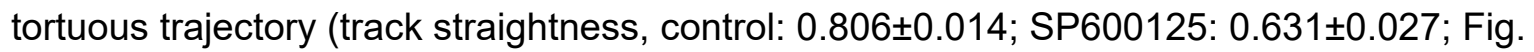
2.2P).

In addition to tracking interneuron movement, the orientation of leading processes was recorded as tangential, diagonal, or radial in each movie frame (control: 3985 frames from 144 tracked cells; SP600125: 6486 frames from 144 tracked cells). The distribution of leading process orientations was significantly different between control and JNK-inhibited conditions (Fig. 2.2Q). Interneurons in control conditions spent $72.1 \pm 2.42 \%$ of their time in a tangential orientation, whereas JNK-inhibited interneurons only spent $42.8 \pm 1.93 \%$ of their time in a tangential orientation. Instead, JNK-inhibited interneurons spent significantly more time in both diagonal $(35.5 \pm 2.45 \%)$ and radial $(21.7 \pm 2.98 \%)$ orientations when compared to controls (21.2 $\pm 2.27 \%$ diagonal, $6.7 \pm 1.28 \%$ radial). The non-tangential trajectories of interneuron leading processes suggest that they are leaving the SVZ stream, which was reflected in a statistically significant shift in the final overall radial distribution of interneurons in the cortical wall (Fig.

2.2R). Post-hoc analyses revealed a significant dispersion of interneurons from the SVZ and an accumulation of interneurons in the cortical plate (CP) and MZ after 12 hours of JNK inhibition. Overall, our results indicate that JNK signaling is required to maintain the tangential orientation and migratory speed of cortical interneurons. Also, the robust infiltration of the CP upon JNK inhibition suggests that downregulation of JNK signaling could mediate the timing of cortical interneuron departure from migratory streams and subsequent entry into the $\mathrm{CP}$.

\section{Migratory stream integrity can be partially restored after removal of JNK inhibition}

In order to determine if the integrity of disrupted migratory streams could be recovered through restoration of JNK signaling, we cultured E14.5 sections for 12 hours in media containing $20 \mu \mathrm{M}$ SP600125, rinsed the slices, and then cultured the slices for an additional 12 
hours in control media. We compared these sections to slices that were treated identically but grown in either control media or media with $20 \mu \mathrm{M}$ SP600125 for the entire 24 hours.

Interneurons in control conditions (Fig. 2.3A-C) predominantly maintained their location in the $\mathrm{MZ}$ and SVZ streams at both the entry zone and the leading edge. Compared to controls, slices treated with $20 \mu \mathrm{M}$ SP600125 (Fig. 2.3D-F) contained cortical interneurons that were widely dispersed from $M Z$ and SVZ streams, accumulating in the CP and VZ regions. When the JNK inhibitor was rinsed out after 12 hours and replaced with control medium ("washout," Fig. 2.3GI), cortical interneurons partially recovered from stream dispersion. Collectively, there were statistically significant changes in the radial distribution of interneurons between all three conditions at both the entry zone and leading edge. As expected, the SVZ showed a significant decrease in the abundance of cortical interneurons in the $20 \mu \mathrm{M}$ SP600125 condition, indicating that JNK inhibition led to evacuation of the SVZ stream. At the entry zone and leading edge locations, there was no statistical difference in the radial distribution of interneurons between control and washout conditions in the $\mathrm{MZ}, \mathrm{CP}$, and $\mathrm{VZ}$ regions (Fig. 2.3K-L), showing a recovery in the placement of interneurons that had accumulated in the CP and VZ when slices were grown in $20 \mu \mathrm{M}$ SP600125. However, interneurons were differentially distributed within the SVZ region, showing incomplete recovery of the SVZ stream.

We also measured the angle of interneuron leading processes in all three conditions (Fig. 2.3J). The overall distribution of leading process angles was statistically different between the control and $20 \mu \mathrm{M}$ SP600125 conditions, but the overall distribution was not significantly different between the control and washout conditions, indicating that interneuron orientation had partially recovered. Thus, during acute JNK inhibition of ex vivo slices, interneurons depart from migratory streams and have misdirected leading processes, suggesting that their ability to correctly navigate their environment is compromised. However, once JNK inhibition is removed, interneurons reorient their trajectories and partially repopulate migratory streams, indicating that their ability to respond to guidance cues located in their normal routes of migration is restored. 


\section{JNK signaling is required for interneurons to enter the cortex at the correct time in vivo}

After finding that pharmacological loss of JNK signaling disrupted migratory streams of cortical interneurons in ex vivo slice cultures, we next wanted to determine if the complete genetic loss of JNK function from interneurons disrupts interneuron migration in vivo. Jnk1;Jnk2 double mutants are early embryonic lethal (Kuan et al., 1999), and combinatorial loss of Jnk1 and Jnk2 has a greater effect on interneuron migration than loss of Jnk1 alone (Myers et al., 2014). We therefore developed two conditional knockout models in which Jnk1 was removed from interneurons using a $D / x 5 / 6-C / E$ driver in either a constitutive Jnk2 null background

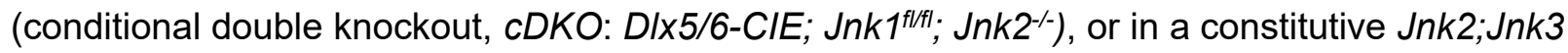

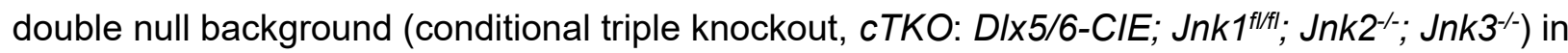
order to completely eliminate JNK signaling in interneurons. In previous work (Myers et al., 2014), we showed that at different rostrocaudal levels, interneurons in $c D K O$ cortices had a delayed entrance into the cortex when compared to embryos heterozygous for the conditional deletion of Jnk1 in a Jnk2 null background (CHKO: D/x5/6-CIE; Jnk $1^{1 / /+}$; Jnk2-- ) at E13.5. In order to determine if the entry of interneurons into the cortex was similarly disrupted in cTKO embryos at E13.5, we compared cTKO, $c H K O$, and $c D K O$ genotypes (Fig. 2.4A-C), and found a statistically significant interaction between genotype and bin location (Fig. 2.4E). Moreover, we observed an allelic dosage effect in the requirement for JNK in interneuron entry into the cortex (Fig. 2.4A-C,E). Post-hoc analyses revealed that there were significant increases in the proportion of interneurons located at the entry zone, as well as significant decreases in the proportion of interneurons reaching more medial cortical positions when Jnk genes were progressively eliminated. These data indicate that genetic removal of Jnk1, Jnk2, and Jnk3 leads to an even more pronounced delay in interneuron entry into the E13.5 cortex than removal of Jnk1 and Jnk2. 
Surprisingly, we found that by E15.5, interneurons had advanced to the same location in the medial cortex of all three genotypes, indicating that cortical interneurons recover from their initial delay at E13.5 (Fig. 2.4F-H, arrows). After observing a similar medial advancement in the cHKO and $c D K O$ cortices at E15.5, we quantitatively assessed the radial distribution of cortical interneurons at lateral, mid, and medial cortical locations, and found no statistical differences between these two genotypes (Fig. 2.S1). The apparent recovery of interneuron advancement and stream integrity in the $c D K O$ cortex compared to the $c H K O$ cortex allowed us to compare interneurons between $c D K O$ and $c T K O$ cortices to determine whether the additional removal of Jnk3 in cTKO embryos further impairs their development.

\section{Genetic loss of JNK signaling alters interneuron distribution and morphology}

Despite an apparent recovery in the medial advancement of interneurons in the E15.5 cortex, we found that the integrity of migratory steams was disrupted in cTKO brains (Fig. 2.5). Gaps were present in the MZ and SVZ streams throughout the lateral to medial extent of the cortex (Fig. 2.5E-F), suggesting that interneurons were missing or displaced from their normal positions. Some $c$ TKO brains had even more severe disruptions, including prominent clusters of interneurons extending from the $\mathrm{MZ}$, or aggregations of interneurons in the subplate region (Fig. 2.S2G,I). cTKO brains that contained large clusters of interneurons also displayed obvious disruptions to the developing cortical wall (Fig. 2.S2H). We excluded cTKO brains from our analyses that contained prominent dipped regions of the cortical plate, since this dysmorphic feature impeded our ability to accurately measure the radial distribution of interneurons. When the remaining $c T K O$ brains were compared to $c D K O$ brains, we found the overall radial distribution of cortical interneurons was significantly altered in the E15.5 cTKO cortical wall (Fig. 2.5I). The lower SVZ contained fewer interneurons in the cTKO cortex, while the upper SVZ contained more, suggesting that interneurons were prematurely departing from the SVZ stream. 
Morphological alterations in the leading processes and/or cell bodies of cortical interneurons could indicate a disruption in their migratory properties (Baudoin et al., 2012; Bellion et al., 2005; Martini and Valdeolmillos, 2010). To determine whether the genetic loss of JNK signaling impacted interneuron morphology, we first measured the length of leading processes of individual interneurons located between the SVZ and MZ streams at E15.5. The average leading process length from $c D K O$ interneurons was statistically longer than in $c T K O$

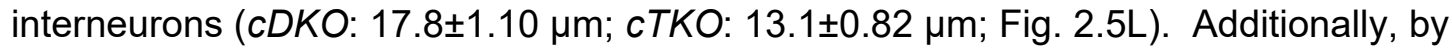
measuring the circularity of interneuron soma, we found cTKO interneurons to be significantly more spherical than interneurons in $c D K O$ cortices (cTKO: 0.741 $\pm 0.0038 ; c D K O: 0.695 \pm 0.010$; Fig. 2.5M). No statistical differences were found in either the area or perimeter of the interneuron cell bodies (data not shown), suggesting that their overall size remained unchanged. Interestingly, in brains with cortical malformations, we found many interneurons with short, blebby leading processes, as well as very spherical cells bodies (Fig. 2.S2I, arrowheads), suggesting interneuron morphology was more affected in these brains. Taken together, our data indicate that complete genetic removal of JNK signaling from cortical interneurons in a Jnk2;Jnk3-deficient environment leads to irregularities in migratory streams that are evident by E15.5 in vivo, as well as morphological alterations to interneurons that are consistent with disrupted migratory properties.

\section{Genetic removal of JNK alters the dynamic migratory properties of cortical interneurons in ex vivo slices}

We next assessed whether the dynamic properties of cortical interneurons changed with genetic ablation of JNK signaling. When organotypic brain slices from E14.5 embryos were imaged for 18 hours ex vivo, many interneurons in $c D K O$ slices maintained tangential progression in migratory streams throughout the entire imaging period (Fig. 2.6A-E; Supplemental Movie 2.3). In contrast, fewer interneurons maintained tangential progression 
over the 18-hour imaging period in cTKO slices (Fig. 2.6F-J; Supplemental Movie 2.4), and many more $C T K O$ interneurons departed from migratory streams in the last 6 hours of imaging (Fig. $2.6 \mathrm{H}-\mathrm{J})$. Only $46.0 \%$ (138/300) of all tracked cortical interneurons remained in the SVZ stream in cTKO slices, while 59.7\% (179/300) remained in $c D K O$ slices. In order to determine if the requirement for JNK differed during the 18 hours of imaging, we analyzed interneuron migration in three, 6-hour intervals (0-6 hours, 6-12 hours, and 12-18 hours, pseudocolored to represent time Fig. 2.6K-M). We found that changes in the dynamic behavior of $c D K O$ and cTKO interneurons were most pronounced during the 12-18 hour time frame, when interneurons normally begin to exit streams.

Unlike with pharmacological JNK inhibition, alterations in the track behavior of $c T K O$ interneurons were not accompanied by statistically significant reductions in migratory speed (cDKO: $\max =123.3 \pm 5.12$, mean=57.9 $\pm 2.76, \min =8.98 \pm 0.84, S D=32.0 \pm 1.54 \mu \mathrm{m} /$ hour; $c$ TKO: $\max =121.7 \pm 3.7, \operatorname{mean}=51.1 \pm 2.02, \min =7.08 \pm 0.96, S D=32.2 \pm 1.01 \mu \mathrm{m} /$ hour; Fig. $2.6 \mathrm{~N}$ ), or a corresponding reduction in overall track length (data not shown). When examining track speed variability however, there was a statistical difference between $c D K O$ and $c T K O$ interneurons (cDKO: 0.642 $\pm 0.024 ; c T K O: 0.752 \pm 0.022$; Fig. 2.6O), suggesting that $c T K O$ interneurons are not traveling at consistent speeds along their migratory tracks. Also, similar to pharmacological inhibition of JNK, cTKO interneurons had an overall shorter displacement (cDKO: 92.3 $\pm 5.09 \mu \mathrm{m}$; cTKO: $75.7 \pm 3.39 \mu \mathrm{m}$; Fig. 2.6P) and less straight trajectories (cDKO: 0.773 $\pm 0.011 ;$ cTKO: $0.718 \pm 0.020$; Fig. 2.6Q). Additionally, when we assessed the amount of time that the leading processes of migrating interneurons were oriented tangentially, diagonally, or radially in each movie frame (cDKO: 2266 frames from 100 tracks; cTKO: 2441 frames from 100 tracks), we found statistically significant differences between the two genotypes (Fig. 2.6R). cTKO interneurons spent significantly less time orientated tangentially $(26.2 \pm 1.94 \%)$ compared to cDKO interneurons $(40.9 \pm 3.73 \%)$, and significantly more time orientated diagonally (cTKO: $51.9 \pm 2.15 \%$; $c D K O: 43.4 \pm 0.84 \%)$. This finding supports our observation that more interneurons 
are leaving streams and are angled upwards towards the CP region of cTKO slices. Together, these data indicate that genetic loss of JNK signaling alters the migratory behavior of cortical interneurons in ex vivo slices of cTKO brains, resulting in tracks with more variable migratory speeds, and more tortuous, misrouted trajectories that increase in severity as development proceeds.

Since our dynamic imaging data revealed that the migratory trajectories of $c T K O$ interneurons worsened over time, we sought to determine if this change in migratory behavior lead to a gradual redistribution of interneurons in ex vivo slices (Fig. 2.7). Initially, no differences in interneuron distribution were observed between E14.5 cDKO and cTKO cortices at the 0-hour time point (Fig. 2.7A-C). However, robust redistribution of $c T K O$ interneurons was evident by the end of the 18-hour imaging period (Fig. 2.7D-F). Post-hoc analyses revealed that there were significant decreases in the abundance of interneurons from the SVZ region of cTKO cortices, and concomitant increases in the abundance of interneurons in the CP region. These data suggest genetic elimination of JNK signaling leads to a premature accumulation of cortical interneurons in the $\mathrm{CP}$.

\section{JNK-depleted interneurons are incorrectly distributed in the early postnatal cortex}

We next wanted to determine whether the early disbanding of interneurons from migratory streams led to long-lasting alterations in their laminar distribution. Although attempts to rear cTKO pups into adulthood proved unsuccessful, we were able to recover live animals at postnatal $(\mathrm{P})$ day 0 in expected Mendelian ratios and analyze their cortices (Fig. 2.8). Similar to $\mathrm{E} 15.5$, some $c$ TKO brains contained cortical malformations at $\mathrm{PO}$, which were variable in size and location (data not shown). We used the mid-cortical position at the level of the anterior commissure to examine the distribution of interneurons, since gross malformations in the cortical plate were not detectable at this location. When we compared the radial distribution of 
interneurons between $c D K O$ and $c T K O$ cortices at PO (Fig. 2.8A-B), we found statistically significant alterations in their placement (Fig. 2.8E). Post-hoc analyses revealed that interneurons in cTKO cortices were most prominently reduced in the VZ/SVZ, and elevated in the lower $\mathrm{CP}$ and $\mathrm{MZ}$ regions. In order to determine whether alterations in interneuron placement were consistent across interneuron subtypes, we examined the positioning of calbindin-labeled cortical interneurons in the P0 cortical wall (Fig. 2.8C-D). Statistically significant alterations in the radial distribution of calbindin-GFP doubled labeled cells were observed between $c D K O$ and $c T K O$ cortices (Fig. 2.8F), with post-hoc analyses identifying significant reductions in the VZ and elevations in the lower CP regions. Interestingly, calbindinGFP double labeled cells did not accumulate in the MZ region, where GFP-positive cells also accumulated in cTKO brains (Fig. 2.8E, bin 1). Indeed, we found a striking increase of GFP. positive calbindin-negative interneurons (open arrowheads in Fig. 2.8C-D) in the $\mathrm{MZ}$ region of cTKO cortices (Fig. 2.8G, bin 1), suggesting that changes to the distribution of the calbindinlabeled cortical interneurons do not fully account for interneuron allocation defects in cTKO brains. Together, our data suggest that migratory anomalies occurring during embryonic development lead to lamination defects that persist until PO in cTKO mice, and that the laminar re-distribution of cortical interneurons in cTKO cortices does not uniformly influence all interneuron subtypes.

\section{Assessing autonomy for JNK function in interneuron migration and allocation}

Although our data support a role for JNK in the migration and laminar positioning of cortical interneurons, it is not clear whether interneurons have a cell-intrinsic requirement for JNK signaling, or whether genetic and pharmacological inhibition of JNK disrupts interneuron migration through non-autonomous mechanisms. In the cTKO model, JNK is completely eliminated from cortical interneurons, however, Jnk2 and Jnk3 are constitutively deleted from the entire animal, which means JNK-depleted interneurons must navigate a partially JNK- 
deficient environment into and within the cortical rudiment. In order to determine whether the Jnk2 and Jnk3 double knockout environment could itself lead to interneuron migration anomalies, we compared the allocation of DIx5/6-CIE cortical interneurons between wild type and Jnk2;Jnk3 double knockout mice at P0. We did not find statistically significant alterations in the distribution of $D / x 5 / 6-C I E$ interneurons between these genotypes (Fig. 2.9A-B,D), suggesting that loss of Jnk2 and Jnk3 is insufficient to alter interneuron allocation. Moreover, when we compared the distribution of interneurons in mice that conditionally eliminated only one copy of Jnk1 from a Jnk2;Jnk3 double knockout background (Fig. 2.9C), we found statistically similar distributions of interneurons to those in wild type and Jnk2;Jnk3 mutant cortices (Fig. 2.9D). However, interneuron distribution in cTKO brains (Fig. 2.8B) was statistically different than wild type (Fig. 2.9A; $\left.F_{(9,60)}=12.73 ; p<0.0001\right)$, Jnk2;Jnk3 double knockout (Fig. 2.9B; $\left.F_{(9,60)}=18.12 ; p<0.0001\right)$, and even Jnk2;Jnk3 double knockouts missing one copy of Jnk1 from DIx5/6-CIE cells (Fig. 2.9C; $\left.F_{(9,60)}=13.39 ; p<0.0001\right)$ by Two-way ANOVA. These findings suggest defects in interneuron positioning are only present when JNK is completely eliminated from interneurons in cTKO brains.

Finally, since pharmacological inhibition of JNK in ex vivo slice cultures disrupts JNK signaling in all cell types within the slice, we asked whether JNK inhibition could impair the migration of interneurons when they are grown alone. We grew explants of medial ganglionic eminence (MGE) tissue in 3D Matrigel cultures for one day, added control medium or medium containing $20 \mu \mathrm{M}$ SP600125, then imaged interneurons live for 12 hours (Fig. 2.9E-G; Supplemental Movies 2.5 and 2.6). By tracking cells in both conditions, we found JNK inhibition in MGE explant cultures to impair the migratory properties of cortical interneurons. When compared to controls, JNK-inhibited interneurons displayed statistically significant alterations in mean and minimum migratory speeds (control: $\max =70.4 \pm 5.11$, mean $=25.8 \pm 1.92$, $\min =3.40 \pm 0.59, S D=17.0 \pm 1.39 \mu \mathrm{m} /$ hour; SP600125: $\max =63.4 \pm 3.53$, mean=19.2 \pm 0.84 , $\min =2.03 \pm 0.24, S D=14.4 \pm 0.77 \mu \mathrm{m} /$ hour; Fig. $2.9 H$ ), speed variability (control: $0.680 \pm 0.021$; 
SP600125: 0.755 \pm 0.026 ; Fig. 2.9I), displacement (control: 90.9 $\pm 7.37 \mu \mathrm{m}$; SP600125: 63.6 \pm 2.59

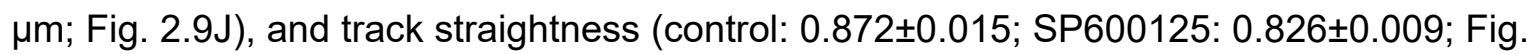
$2.9 \mathrm{~K})$. These findings suggest that the migratory behavior of cortical interneurons at least partially relies on a cell-intrinsic requirement for JNK signaling. 


\section{Discussion}

We found that tangential progression of cortical interneurons in migratory streams depends on the JNK signaling pathway. Pharmacological inhibition of JNK signaling in E14.5 slices resulted in dispersion of cortical interneurons from pre-formed migratory streams, which was partially recoverable upon removal of the inhibitor. Live imaging revealed that acute JNK inhibition led to rapid stream departure, as JNK-inhibited cortical interneurons switched from tangential to radial modes of migration, exited the SVZ stream, and infiltrated the cortical plate. To genetically eliminate JNK from interneurons, we developed conditional triple knockout (cTKO) mice, which removed Jnk1 from interneurons of Jnk2;Jnk3 double knockouts. Live imaging of $c T K O$ slice cultures at E14.5 recapitulated our ex vivo pharmacologic data by showing an increase in non-tangential migratory trajectories, evacuation of the SVZ stream, and premature accumulation of interneurons in the cortical plate. In cTKO cortices in vivo, interneurons were delayed in their arrival to the cortex at E13.5 and were displaced from migratory streams at E15.5. At P0, we found that interneurons in cTKO cortices had vacated the VZ/SVZ and overpopulated deeper layers of the CP and MZ, and that distinct subtypes of cortical interneurons accumulated differently in the JNK-deficient cortical wall. Finally, we provided genetic and pharmacological evidence that disrupted migration and allocation of cortical interneurons at least partially relies on cell-intrinsic requirements for JNK signaling. Together, our results suggest that JNK signaling maintains the integrity of cortical interneuron migratory streams and enables the correct positioning of interneurons in the cortical wall.

\section{Molecular mechanisms underlying cortical plate invasion by interneurons}

The JNK signaling pathway might facilitate interneuron departure from migratory streams by intersecting with other known mediators of cortical plate invasion. The chemokine Cxcl12 is expressed along the migratory routes of cortical interneurons and helps to establish and maintain migratory streams in the developing cortex (Stumm et al., 2003; Tham et al., 2001; 
Tiveron et al., 2006). Chemoattraction to $\mathrm{Cxcl} 12$ is mediated by the activity of two chemokine receptors, Cxcr4 and Cxcr7, which are expressed by migrating cortical interneurons (LopezBendito et al., 2008; Sanchez-Alcaniz et al., 2011; Wang et al., 2011). Pharmacological loss of Cxcr4 function in other studies (Lysko et al., 2011; Wang et al., 2011), and the JNK pathway here, results in migratory stream departure and the precocious entry of interneurons into the cortical plate. Cxcr4, Cxcr7, and Cxcl12 null mice have disrupted migratory streams and persistent alterations to cortical interneuron distribution (Abe et al., 2014; Lopez-Bendito et al., 2008; Sanchez-Alcaniz et al., 2011; Wang et al., 2011), which closely parallel our findings in cTKO cortices. Additionally, in both Cxcr7 null (Wang et al., 2011) and cTKO mice, interneurons have morphological alterations, including shorter, less tangentially-oriented leading processes. These similarities, along with other studies indicating that Cxcr4 signaling can activate JNK to induce migration in non-neuronal cells (Decaillot et al., 2011; Liao et al., 2015), suggest that JNK may act downstream of chemokine signaling to modulate the formation and maintenance of migratory streams. Thus, as interneuron responsiveness to Cxcl12 diminishes, a key feature thought to mediate migratory stream departure (Li et al., 2008), JNK levels may decline to enable the tangential to radial switch.

Mechanisms outside of chemokine signaling also appear to regulate cortical plate invasion. Connexin43 (Cx43), a gap junction protein, participates in the tangential to radial transition by promoting adhesion between cortical interneurons and radial glial cells (Elias et al., 2010). Studies in cardiomyocytes suggest that JNK activation downregulates the expression of Cx43 (Petrich et al., 2002). In interneurons, downregulation of Cx43 decreases interneuron entry into the cortical plate (Elias et al., 2010). Therefore, developmental reduction of JNK activity could promote migratory stream exit by increasing the expression of Cx43 in migrating cortical interneurons. Additionally, other extrinsic factors may reinforce cortical plate invasion. For example, Sonic hedgehog (Shh) is expressed at low levels in the developing cortex, and treatment of cortical slices with Shh results in stream dispersion and cortical plate accumulation 
(Baudoin et al., 2012), reminiscent of the stream departure seen after JNK inhibition. Similarly, Neuregulin 3 (Nrg3) is expressed by excitatory neurons in the developing cortex (Bartolini et al., 2017), and is a ligand for ErbB4, a receptor tyrosine kinase expressed by migrating cortical interneurons (Flames et al., 2004; Yau et al., 2003). Overexpression of Nrg3 promotes migratory stream departure, and conditional deletion of $\mathrm{Nrg} 3$ or ErbB4 alters the laminar

allocation of interneurons in the postnatal cortex (Bartolini et al., 2017). Thus, it is possible that activation of these pathways reduces JNK activity in cortical interneurons, or that reduced JNK activity facilitates Shh- and ErbB4-mediated migratory stream departure, but additional experiments are needed to test these models.

\section{Diverse requirements for JNK during cortical interneuron migration}

Complex migratory decisions, such as choosing when and where to depart from migratory streams, likely involve the coordination of extrinsic and intrinsic molecular signals. Since JNKs are expressed in both interneurons and other cells of the cortical environment (Myers et al., 2014), JNK signaling may influence interneurons through both autonomous and non-autonomous mechanisms. One means by which JNK could exert a cell autonomous influence on cortical interneuron migration is through modulation of the cytoskeleton. For instance, doublecortin, a microtubule-binding protein known to regulate leading process branching and guided migration of cortical interneurons (Friocourt et al., 2007; Kappeler et al., 2006), is a downstream target of JNK signaling (Gdalyahu et al., 2004; Jin et al., 2010). Additionally, p27 ${ }^{\mathrm{kip} 1}$ is a microtubule-associated protein that coordinates microtubule polymerization and actomyosin contraction to attune leading process branching and nucleokinesis (Godin et al., 2012). Conditional deletion of p27 kip1 from post-mitotic cortical interneurons delays cortical entry at E12.5 (Godin et al., 2012), similar to our observations following loss of JNK. During cancer cell migration, JNK signaling regulates cell-cell adhesions via p27 ${ }^{\text {kip1 }}$ phosphorylation (Kim et al., 2012), suggesting a link exists between JNK and p27kip1 
in migratory cells. Molecular mechanisms underlying the connections between JNK signaling, cytoskeletal modulators, and other intrinsic features of interneuron migration remain to be determined.

In addition to intrinsic mechanisms, extrinsic influences on cortical interneuron migration may also be controlled by JNK. Indeed, we found greater disruptions to cortical interneuron migratory properties when ex vivo slices were treated with a JNK inhibitor than when interneurons were inhibited in the absence of all other cortical cells (compare Fig. 2.2M-P to Fig. $2.9 \mathrm{H}-\mathrm{K})$. This suggests that disruptions to JNK signaling may alter the environment that cortical interneurons travel through. Migrating cortical interneurons normally rely on cellular and molecular interactions with other cells to navigate into and within the cerebral cortex. For instance, interneurons often reorient their mode of migration from tangential to radial after making contact with radial glia in the cortical wall (Yokota et al., 2007). Furthermore, disruptions to radial glia (Haubst et al., 2006; Talebian et al., 2017), cortical intermediate progenitors (Abe et al., 2015), cortical excitatory neurons (Hevner et al., 2004; Lodato et al., 2011; Pla et al., 2006), microglia (Squarzoni et al., 2014), blood vessels (Barber et al., 2018), and thalamocortical axons (Zechel et al., 2016) all have a non-autonomous effect on the migration of cortical interneurons. Therefore, if JNK activity is required for the normal development of these cellular components of the cortical environment, as is the case for radially migrating cortical excitatory neurons (Westerlund et al., 2011; Zhang et al., 2016), JNK disruption may nonautonomously impact interneuron migration.

In pharmacologically treated and genetically manipulated ex vivo slices, interneurons adopted non-tangential trajectories and prematurely departed from migratory streams, indicating that both approaches used to eliminate JNK function yielded consistent results. However, we found that interneurons in JNK-inhibited slices displayed greater alterations in migratory properties, which led to faster and more complete disruption of migratory streams. This could be explained by inherent differences in acute pharmacologic inhibition, where JNK signaling is 
abruptly abrogated, versus chronic genetic loss, where JNK removal may be compensated for over time. Alternatively, since all three Jnk proteins are globally inhibited after SP600125 treatment while Jnk1 is retained in DIx5/6-CIE-negative cells of cTKO cortices, Jnk1 function in non-interneuronal cells of the genetic model may lessen the severity of interneuron phenotypes. Thus, regardless of how differences between pharmacologic and genetic manipulations originate, JNK function in other cell types should be carefully considered.

Finally, our data suggest that Jnk2 and Jnk3 are not critical for the normal allocation of cortical interneurons at P0. However, we cannot exclude the possibility that the Jnk2 and Jnk3deficient environment of $c T K O$ brains does not further compromise the migration and allocation of JNK-depleted interneurons. Moreover, since JNK is conditionally deleted in ventral forebrain progenitors of the $c T K O$ brain, we cannot exclude the possibility that aberrant subcortical migration or delayed entry into the cortical rudiment impacts subsequent allocation of interneurons in the cortical wall. More sophisticated in utero manipulation experiments designed to only eliminate JNK activity from cortical interneurons after they have entered the cortex are needed to address the autonomous and temporal requirements for JNK in interneuron migration and allocation.

\section{Conclusions and Future Perspectives}

We found a novel requirement for JNK signaling in cortical interneuron stream maintenance, interneuron migratory behavior and morphology, and the laminar distribution of interneurons in the cortical wall. These findings are significant, since even minor disruptions to the migration of cortical interneurons have been implicated in the etiology of severe neurological and neuropsychiatric disorders (Dubos et al., 2018; Meechan et al., 2012a; Volk et al., 2015). Therefore, elucidating upstream activators and downstream effectors of JNK signaling in cortical interneurons will be essential for determining extrinsic and intrinsic functions of JNK in cortical 
development, and could help to unravel complex neurodevelopmental disorders that impinge upon the formation and function of the cerebral cortex.

\section{Materials and Methods}

\section{Animals}

Mice (Mus musculus) were housed and cared for by the Office of Laboratory Animal Resources at West Virginia University. Timed-pregnant dams (day of vaginal plug = embryonic (E) day 0.5 ) were euthanized by rapid cervical dislocation, and mouse embryos were immediately harvested for tissue culture or histological analyses. For ex vivo pharmacological slice culture experiments, CF-1 (Charles River) dams were crossed to hemizygous D/x5/6-CreIRES-EGFP (DIx5/6-CIE; (Stenman et al., 2003)) males maintained on a C57BL/6J (Stock \# 000664, The Jackson Laboratory) background to achieve timed pregnancies at E14.5. For medial ganglionic eminence (MGE) explant assays, C57BL/6J dams were crossed to D/x5/6CIE males. For in vivo genetic knockout experiments, as well as ex vivo slice cultures using genetic knockout material, new mouse strains were established and maintained on a C57BL/6J background. Floxed Mapk8 $8^{\operatorname{tm} 1 R j d}$ mice (Jnk1 $1^{f / f f l}$; (Das et al., 2007); kindly provided by Dr. Roger Davis) were bred with Mapk9tm1Flv (Jnk2-/-; Stock \# 004321, The Jackson Laboratory), Mapk10 tm1F/v (Jnk3--; Stock \# 004322, The Jackson Laboratory), and/or D/x5/6-CIE mice to generate breeding stock for genetic experiments. Jnk $1^{f / f l l}$; Jnk2-/- dams were mated with D/x5/6CIE; Jnk1 ${ }^{f / /+}$; Jnk2 $2^{-/-}$males to generate conditional heterozygote knockout mice (cHKO: DIx5/6-

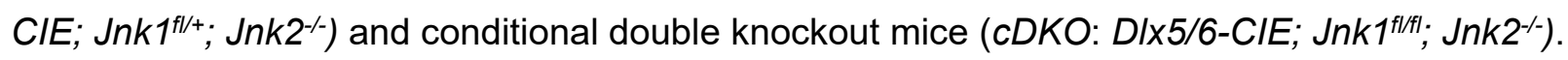

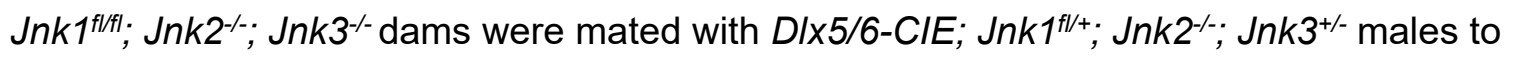
generate conditional triple knockout mice (cTKO: DIx5/6-CIE; Jnk $1^{f / / f l} ; J n k 2^{-/ / ;} J n k 3^{-/-}$), as well as

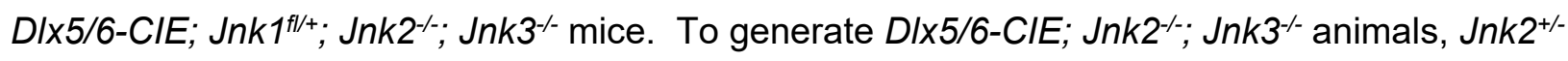

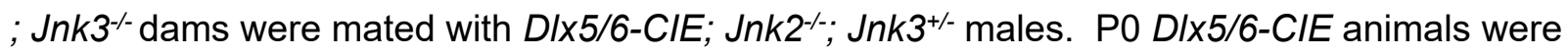


generated by crossing C57BL/6J dams with a DIx5/6-CIE male. All animal procedures were performed as approved by the Institutional Animal Care and Use Committee at West Virginia University.

\section{Organotypic Slice Cultures}

DIx5/6-CIE positive embryos were collected at E14.5 and dissected in ice-cold complete HBSS (cHBSS; Tucker et al., 2006). The embryonic brains were embedded in a solution of $3 \%$ low melting point agarose (Fisher Scientific BP165-25) in cHBSS, sectioned coronally into 300 $\mu \mathrm{m}$ slices on a Leica VT1000 S vibratome, and transferred to poly-L-lysine/laminin coated transwell membrane inserts in 6-well plates (BD Falcon; Polleux and Ghosh, 2002). Each well was filled with $1.8 \mathrm{~mL}$ of slice culture media (Tucker et al., 2006) containing either dimethyl sulfoxide (DMSO, Sigma D2438) as a vehicle control or the pan-JNK inhibitor SP600125 (Enzo Life Sciences BML-EI305-0010) at a final concentration of $20 \mu \mathrm{M}$ or $40 \mu \mathrm{M}$, as previously described (Myers et al., 2014). Slices were grown for 12 hours for the pharmacological dosage experiment (Fig. 2.1). For the washout experiment (Fig. 2.3), slices were grown for 12 hours, the media containing the vehicle or the inhibitor was replaced, and the slices were grown for an additional 12 hours.

\section{D Matrigel Assay}

DIx5/6-CIE positive embryos were collected at E14.5. MGE tissue was micro-dissected in ice-cold cHBSS, cut into small explants, and stored in cHBSS on ice. Matrigel (Corning \#356237) was combined 1:1 with serum free media (Polleux and Ghosh, 2002), and $150 \mu \mathrm{L}$ of the Matrigel mixture was added to each well of an 8-well chamber coverslip slide (Thermo Fisher 155411) on ice. Two to three MGE explants were placed at the bottom of each well near the coverslip. The slide was then transferred to a tissue culture incubator at $37^{\circ} \mathrm{C}$ with $5 \% \mathrm{CO}_{2}$ for 30 minutes to solidify the Matrigel mixture with the embedded explants. Warm serum free media was added to each well and the explants were cultured for 24 hours. After 24 hours, 
serum free media containing either control (DMSO) or SP600125 at a final concentration of 20 $\mu \mathrm{M}$ was added to each well immediately before imaging.

\section{Live Imaging}

Live-vibratome slices of E14.5 brains (prepared as above) were transferred to Millicell inserts (Millipore PICMORG50) in a FluoroDish (World Precision Instruments FD35-100) either filled with $1.6 \mathrm{~mL}$ slice culture media for genetic knockout experiments, or filled with $1.6 \mathrm{~mL}$ slice culture media containing either control (DMSO) or $20 \mu \mathrm{M}$ SP600125 for pharmacological experiments. FluoroDish preparations, or Matrigel slides (prepared as above), were transferred to a Zeiss 710 confocal microscope equipped with stable environmental controls maintained at $37^{\circ} \mathrm{C}$ with $5 \%$ humidified $\mathrm{CO}_{2}$. Multi-position time-lapse z-series were acquired every 10 min for 12-24 hours with a LD Plan-Neofluar 20x/0.4 Korr objective lens for slices, and with a 20x PlanApo lens for the Matrigel experiments. The field of view was centered on the mid-point of the cortical wall for slices, and at the leading edge of interneuron outgrowth for the Matrigel explants. Tissue samples were collected from embryos for retrospective genotype identification.

\section{Live Imaging Analyses}

4D live-imaging movies were analyzed using Imaris (Version 9.3.1) software by Bitplane. In slice culture assays, interneurons were tracked from left, center, and right portions of the SVZ stream from movies recorded at the mid-cortical position. For selection, interneurons had to originate in the SVZ stream, migrate for at least 2 hours, and be clearly distinguishable from surrounding cells. For Matrigel assays, interneurons had to migrate for a minimum of 4 hours. All cell tracks were stopped when interneurons were no longer visible/distinguishable from nearby cells, if they stopped moving for a period of 60 min while being tracked, or when the movie ended. For the pharmacological experiments, 12 interneurons were tracked from each of the 12 movies per condition $(n=12)$, for a total of 144 tracks per condition. Movies were generated from at least 7 different embryos per condition over 3 experimental days. For the 
genetic experiments, 10 interneurons were tracked from each of the 10 movies per genotype $(n=10)$, for a total of 100 tracks per genotype in each of the three 6-hour segments $(300$ tracks/genotype). Movies were generated from 5 different brains per genotype, collected over 4 experimental days. For the Matrigel experiment, 10 interneurons were tracked from each of the 8 movies per condition $(n=8)$, for a total of 80 tracks per condition, collected from 3 experiments.

All tracks from each movie were averaged together for dynamic analyses. Cortical interneurons were tracked using the Spots feature of Imaris to capture migratory speed, distance, displacement, and track straightness data, which were analyzed by two-tailed Student's $t$-tests. Displacement was normalized to the minimum track length (2 hours slice culture, 4 hours Matrigel) to account for differences in the total times of tracked cells. In addition, in the slice culture experiments, the direction of the leading processes of tracked cells was recorded each frame as tangential, diagonal, or radial orientation. Overall differences in orientation were determined by Two-way ANOVA followed by Fisher's LSD post-hoc analyses.

\section{Tissue Processing, Cryosectioning, and Immunohistochemistry}

E13.5 or E15.5 brains were dissected in phosphate-buffered saline (PBS; 136.9mmol $\mathrm{NaCl}, 2.683 \mathrm{mmol} \mathrm{KCl}, 4.290 \mathrm{mmol} \mathrm{Na}_{2} \mathrm{HPO}_{4} 7 \mathrm{H}_{2} \mathrm{O}, 1.470 \mathrm{mmol} \mathrm{KH}_{2} \mathrm{PO}_{4}$ ) and immersion fixed with $4 \%$ paraformaldehyde (PFA) in PBS overnight at $4^{\circ} \mathrm{C}$. Postnatal $(\mathrm{P})$ day 0 pups were anesthetized on ice and transcardially perfused with chilled PBS followed by $4 \%$ PFA, and brains were dissected in PBS and immersion fixed overnight at $4^{\circ} \mathrm{C}$. Samples were rinsed with PBS and progressed through a sucrose series (10\%, 20\%, and $30 \%)$ prior to embedding. For fixed analyses of ex vivo slice cultures, slices were rinsed in PBS, fixed overnight in $4 \%$ PFA at $4^{\circ} \mathrm{C}$, passaged through sucrose, and re-embedded in agar sucrose $(3.5 \%$ agar $+8 \%$ sucrose $)$ prior to removal from the transwell membrane. Both brains and slices were embedded in Tissue Freezing Medium (VWR 15146-019), flash frozen in liquid nitrogen-cooled 2-methyl butane (Fisher Scientific $03551-4$ ), and stored at $-80^{\circ} \mathrm{C}$. Frozen brains and slice cultures were 
sectioned coronally at $12 \mu \mathrm{m}$ on a Leica cryostat (CM 3050S), collected in series onto Superfrost Plus slides (Fisher Scientific 12-550-15), and stored at $-20^{\circ} \mathrm{C}$ prior to use. Slides were rehydrated with PBS for 20 mins and blocked for 2 hours in permeability solution (Myers et al., 2014) with 5\% normal goat serum. Primary antibodies including Chicken anti-GFP (1:1500, Abcam ab13970) and Rabbit anti-Calbindin (1:2000, Swant CB38) were diluted in permeability solution, applied to the slices, and incubated overnight at $4^{\circ} \mathrm{C}$. Slices were thoroughly rinsed in PBS and incubated with secondary antibodies including Alexa 488 conjugated goat anti-chicken (1:4000, Invitrogen) and Alexa 546 conjugated goat anti-rabbit (1:2000, Invitrogen) diluted in permeability solution at room temperature for 2 hours. Hoechst (Thermo Scientific 62249, $1 \mu \mathrm{g} / \mathrm{ml}$ ) was used as a nuclear counterstain. Slices were rinsed in PBS, mounted in an aqueous mounting medium containing an anti-fade reagent, and stored at $4^{\circ} \mathrm{C}$.

\section{Imaging and Quantification of Cryosectioned Slices}

Imaging. Immunofluorescently-labeled cryosections of embryonic brain slices were imaged on a Zeiss 710 confocal microscope with a 20x Plan-Apo objective lens. P0 sections were imaged using an Olympus VS120 Slide Scanner with a UPLSAPO 10x objective. Confocal and slide scanned images were uniformly adjusted for levels, brightness, and contrast in Adobe Photoshop.

Interneuron distribution. For quantifying radial distribution of interneurons, cropped regions of the cortical wall were equidistantly segmented into 10 bins from pial to ventricular surfaces (as in Fig. 2.S1C-D). For quantifying interneuron entry into the cortex, the E13.5 cortical rudiment was divided into 5 equidistant bins (lateral to medial, as in Fig. 2.4D). For all experiments, the numbers of cells present in each equidistant bin were counted and their percentile distributions across all bins were determined for each tissue section. Bin distributions were averaged across sections of the same treatment group or genotype, and statistical significances were determined by Two-way ANOVA followed by Fisher's LSD post-hoc analyses (GraphPad Prism 8). For 
E13.5 and E15.5 in vivo analyses, cropped regions from 2 hemi-sections were quantified at 4 predefined rostrocaudal locations, for a total of 8 sections per brain ( $n=5$ brains/genotype). At P0, 3 hemi-sections were selected from slices containing the anterior commissure region $(n=3$ or $n=5$ brains/genotype). In pharmacological experiments, 4 sections were used from each treated slice ( $n=5$ brains/condition).

Interneuron morphology and leading process orientation. Interneuron morphologies were quantified by measuring the circularity of cell bodies and the length of leading processes in Adobe Photoshop. Leading process length was measured from clearly distinguishable cells located between the SVZ and MZ streams as a vector from the end of the cell body to the furthest point of the longest process. Two-tailed Student's $t$-tests were used to evaluate the statistical significance of morphological measurements between cells analyzed in each genotype. For both measurements, cells were selected from 2 hemi-sections per brain, at the same defined rostrocaudal location ( $n=5$ brains/genotype). Orientation of leading processes was determined by measuring the angle of displacement from the tangential direction, which was defined as 0 degrees. All angle measurements ( $n=5$ brains/condition) were grouped into 12 different bins of 30 degrees each, and statistical significances were determined by ChiSquare analysis followed by Fisher's LSD post-hoc analyses (GraphPad Prism 8).

\section{Acknowledgements}

We would like to thank Katie Padgett and Kelly Stake for their assistance in sample processing and data analysis, as well as Amanda Ammer and Karen Martin for their excellent microscopy support. Imaging experiments were performed in the WVU Imaging Facilities, which have been supported by the WVU Cancer Institute, the WVU HSC Office of Research and Graduate Education, and NIH grants P20RR016440, P30GM103488, P20GM121322, U54GM104942, P20GM10343, and P30GM103503. 


\section{Competing Interests}

No competing interests declared.

\section{Author Contributions}

Conceptualization: A.K.M. and E.S.T.; Methodology: A.K.M., J.G.C., and E.S.T.; Formal analysis: A.K.M., J.G.C., S.E.S., and J.P.S.; Investigation: A.K.M., J.G.C., J.P.S., S.E.S., and C.A.S.; Writing- original draft: A.K.M.; Writing- review and editing: J.G.C., A.K.M., S.E.S., and E.S.T.; Visualization: J.G.C., A.K.M., and S.E.S.; Supervision: E.S.T.; Funding Acquisition: E.S.T.

\section{Funding}

This work was supported by the National Institutes of Health grant R01NS082262 to EST. 


\section{Figures}

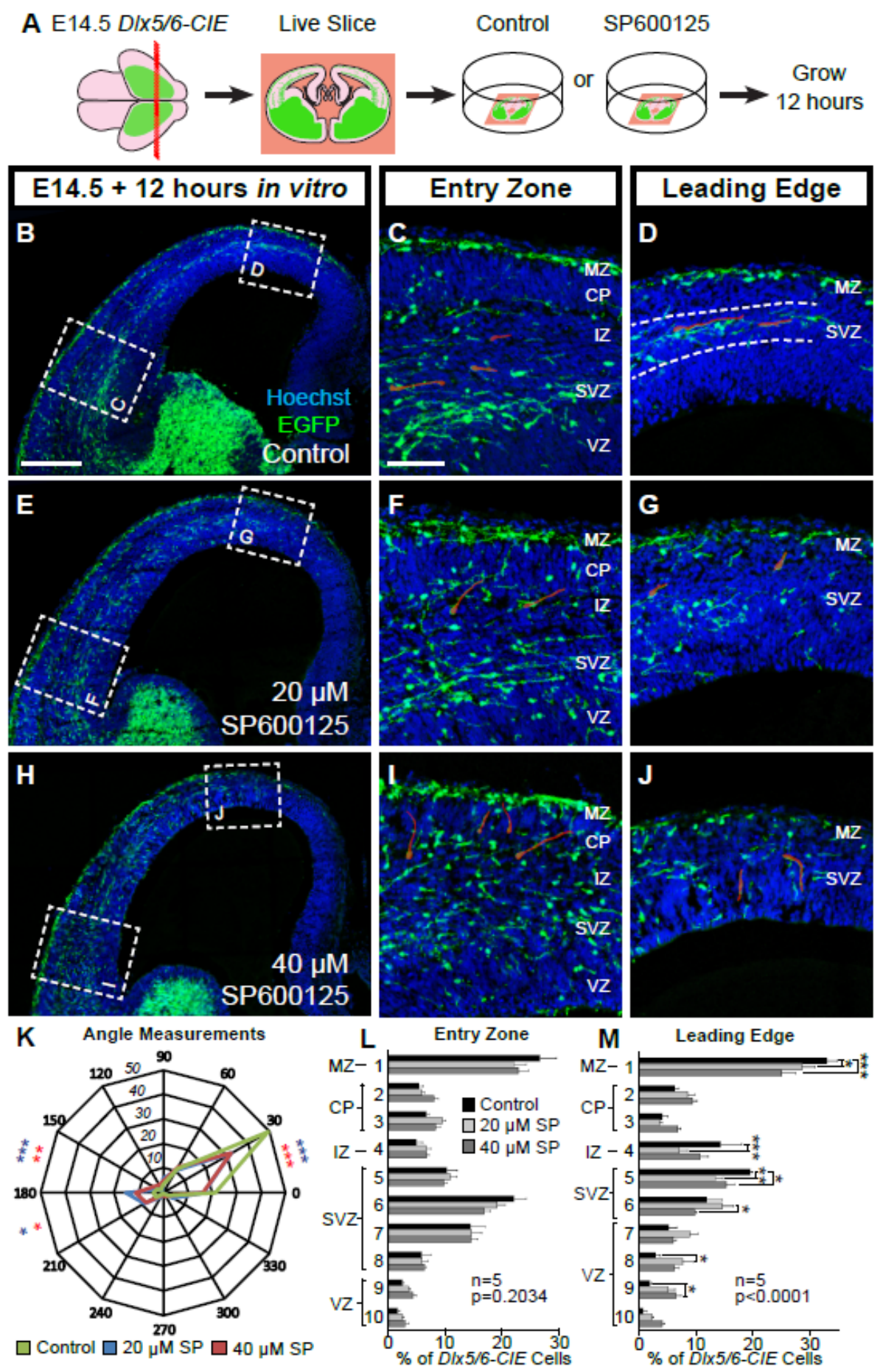

Figure 2.1: JNK activity is required for cortical interneurons to remain tangentially

oriented in migratory streams. (A) Schematic diagram illustrating ex vivo slice culture of

E14.5 D/x5/6-CIE brains in control or SP600125-treated conditions. (B-D) In control slices, 
cortical interneurons travel tangentially in migratory streams in the MZ and SVZ (dashed lines, D). (E-J) Streams are disrupted with $20 \mu \mathrm{M}(\mathrm{E}-\mathrm{G})$ and $40 \mu \mathrm{M}(\mathrm{H}-\mathrm{J})$ SP600125. Representative interneurons are highlighted in red. (K) Quantification of leading process angles. Interneurons are more tangentially oriented in control (200 cells) compared to $20 \mu \mathrm{M}$ (178 cells; $p=0.0009)$ and $40 \mu \mathrm{M}$ (256 cells; p=0.0014) SP600125 conditions (Chi-Square). (L-M) Interneurons in JNK-inhibited conditions are significantly displaced at the leading edge (Two-way ANOVA: $\left.F_{(18,120)}=3.582 ; p<0.0001\right)$. All analyses were performed on $n=5$ brains/condition from at least 4 experimental days. Error bars represent mean \pm s.e.m., post-hoc by Fisher's LSD ${ }^{* * *} p<0.001$, ${ }^{* *} p<0.01,{ }^{*} p<0.05$. Scale bars: $250 \mu \mathrm{m}(\mathrm{B}, \mathrm{E}, \mathrm{H}) ; 75 \mu \mathrm{m}(\mathrm{C}-\mathrm{D}, \mathrm{F}-\mathrm{G}, \mathrm{I}-\mathrm{J})$. 

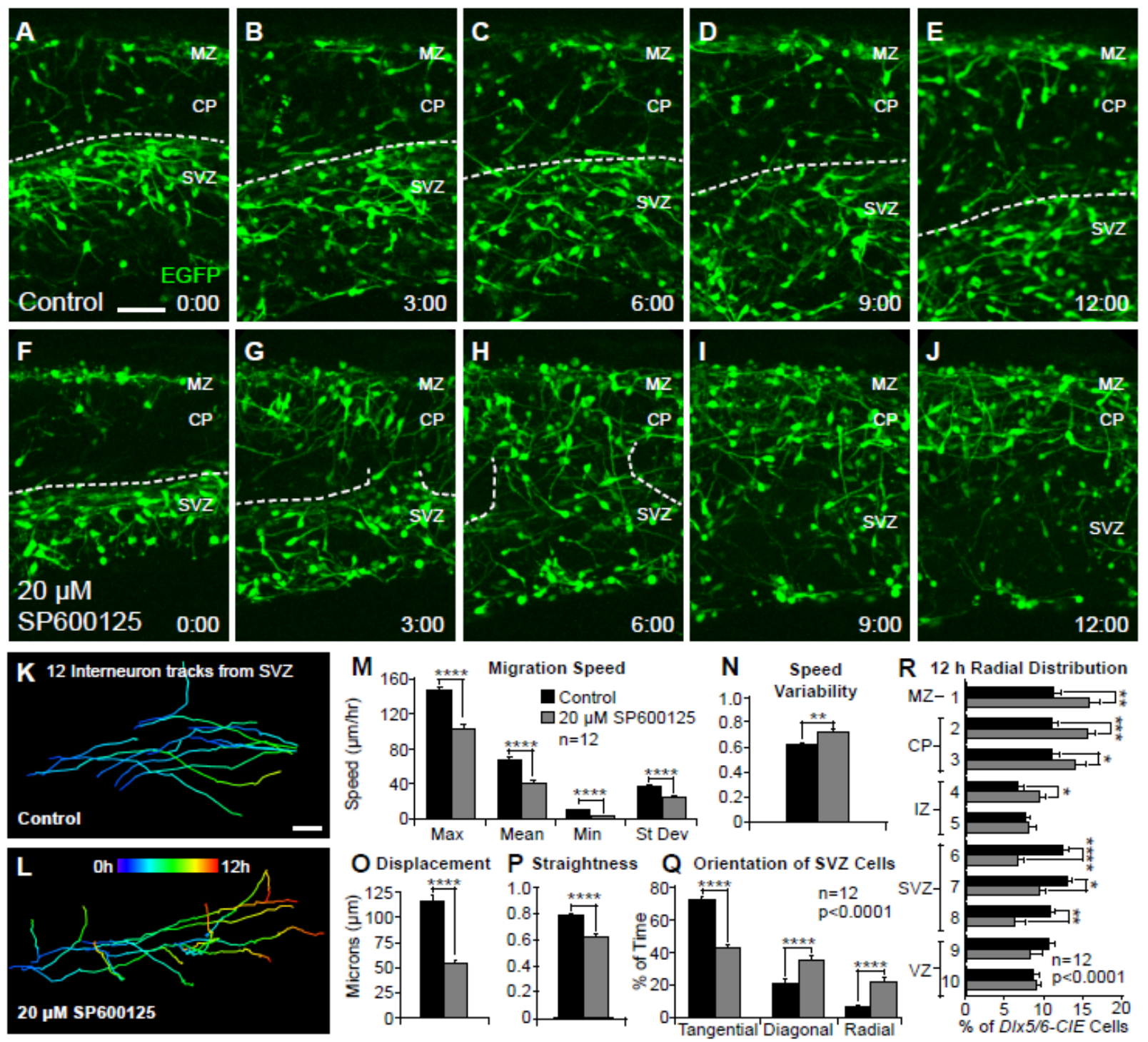

Figure 2.2: Dynamic migratory properties of cortical interneurons are perturbed following pharmacological inhibition of JNK. (A-J) Movie frames from E14.5 D/x5/6-CIE cortices imaged under control (A-E) or $20 \mu \mathrm{M}$ SP600125 (F-J) conditions for 12 hours ex vivo. Dashed lines follow the top of the SVZ stream in control conditions (A-E), and breakdown of the SVZ stream in JNK-inhibited conditions (F-J). (K-L) Tracks (pseudo-colored by time) from 12 interneurons in control (K) or $20 \mu \mathrm{M}$ SP600125 (L) conditions. For each condition, 12 interneurons were tracked from $n=12$ movies (144 tracks/condition), generated from at least 7 different embryos over 3 experimental days. (M-P) Quantification of interneuron migratory 
properties (Student's t-tests). (Q) Quantification of interneuron leading process orientations (Two-way ANOVA: $\left.F_{(2,66)}=61.71 ; p<0.0001\right)$. (R) Quantification of the radial distribution of interneurons at 12 hours (Two-way ANOVA: $F_{(9,220)}=7.651 ; p<0.0001$ ). Error bars represent mean \pm s.e.m., post-hoc by Fisher's LSD ${ }^{* * *} p<0.0001,{ }^{* * *} p<0.001,{ }^{* *} p<0.01,{ }^{*} p<0.05$. Scale bars: $50 \mu \mathrm{m}$. 

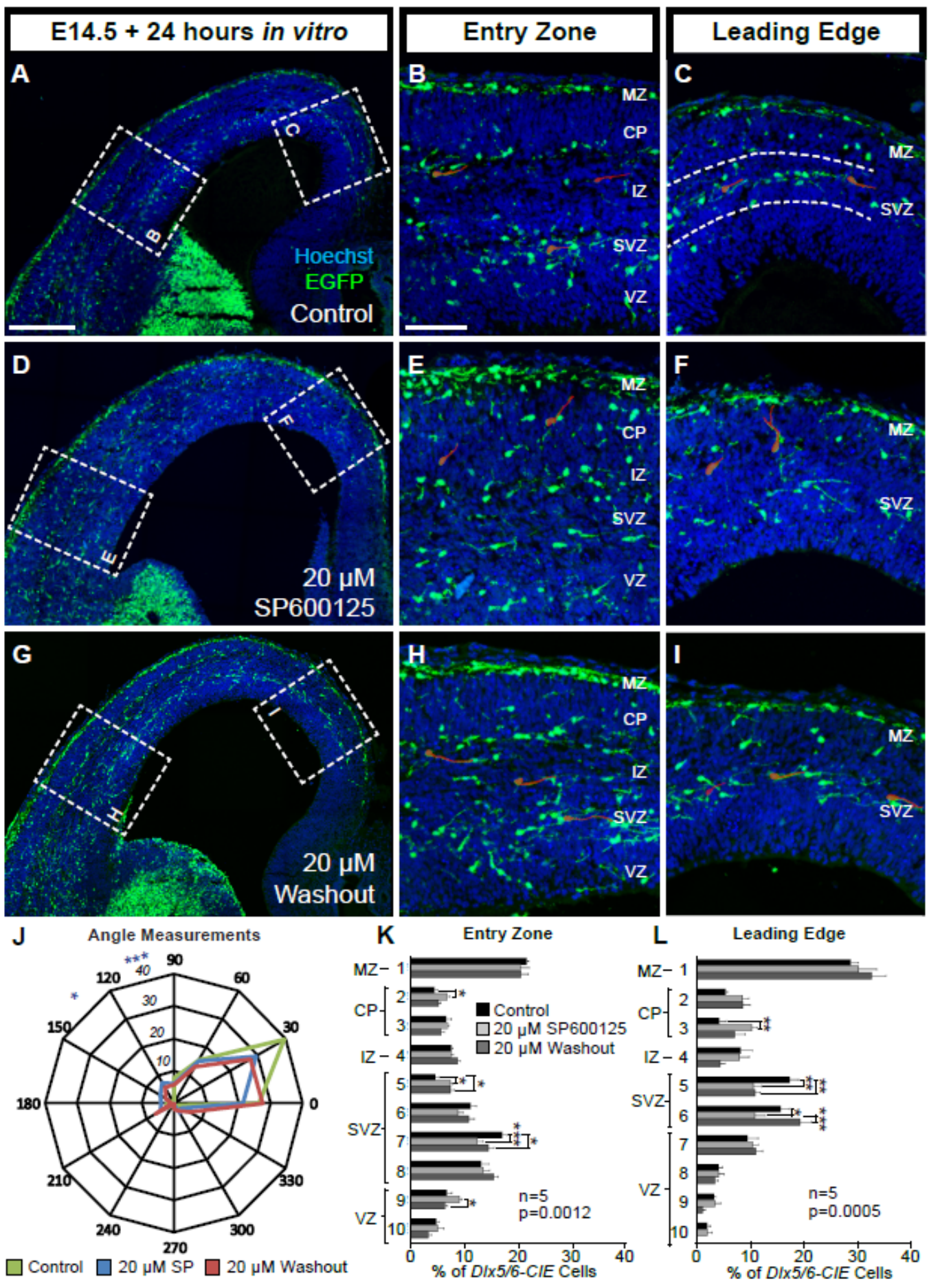

Figure 2.3: Migratory stream integrity partially recovers after removal of JNK inhibitor.

(A-C) Migratory streams remain intact (dashed lines, C) when slices are grown in control conditions for 24 hours. (D-F) MZ and SVZ streams are disrupted when slices are grown in 20 MM SP600125. (G-I) Partial recovery of the SVZ stream is observed when slices are grown in 
$20 \mu \mathrm{M}$ SP600125 for 12 hours, rinsed, and then grown in control media for an additional 12 hours ("washout"). Representative interneurons are highlighted in red. (J) Quantification of leading process angles. Differences are found between control (129 cells) and $20 \mu \mathrm{M}$ SP600125 (178 cells; $p=0.031$ ), but not between the control and washout (106 cells; $p=0.065$ ) conditions (Chi-Square). (K-L) Stream dispersion partially recovers in the washout condition (Two-way ANOVA: entry zone $F_{(18,120)}=2.579, p=0.0012$; leading edge $F_{(18,120)}=2.785 ; p=0.0005$ ). All analyses were performed on $n=5$ brains/condition from 5 experimental days. Error bars represent mean \pm s.e.m., post-hoc by Fisher's LSD ${ }^{* * *} p<0.001,{ }^{* *} p<0.01,{ }^{*} p<0.05$. Scale bars: $250 \mu \mathrm{m}(\mathrm{A}, \mathrm{D}, \mathrm{G}) ; 75 \mu \mathrm{m}(\mathrm{B}, \mathrm{C}, \mathrm{E}, \mathrm{F}, \mathrm{H}, \mathrm{I})$. 

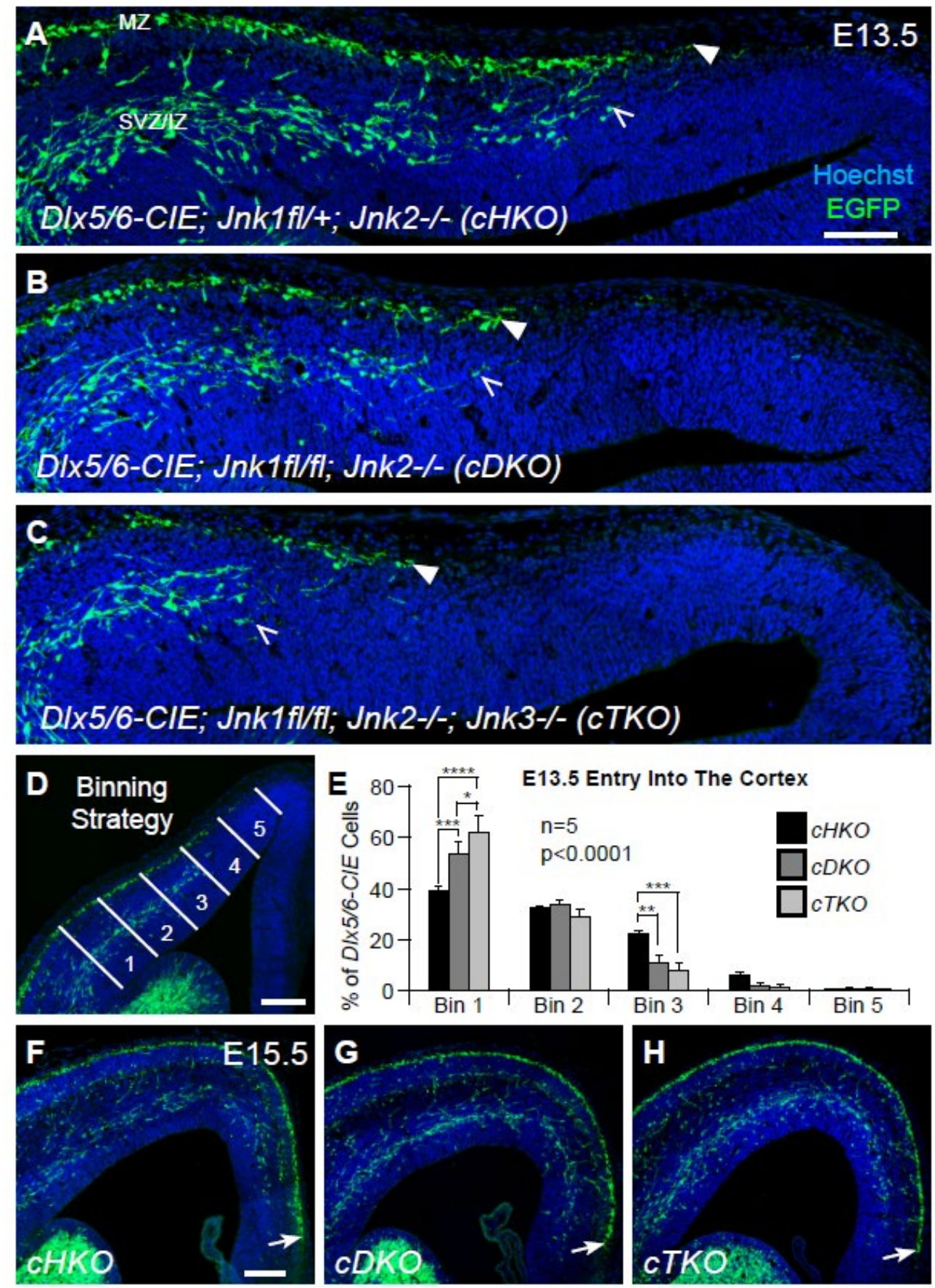

Figure 2.4: Arrival of interneurons into the cortex is transiently delayed by genetic removal of JNK function in vivo. (A-C) At E13.5, interneuron entry (MZ stream-closed arrowheads, SVZ stream-open arrowheads) is progressively delayed into the cortex with the stepwise removal of JNK function in vivo. (D-E) Quantification of interneuron distribution in 5 equidistant bins (lateral to medial) was performed on $n=5$ brains/genotype (Two-way ANOVA: 
$\left.F_{(8,60)}=7.52 ; p<0.0001\right)$. ( $\left.F-H\right)$ Interneurons in all three genotypes have advanced to a similar location in the medial cortical wall by E15.5 (arrows). Error bars represent mean \pm s.e.m., posthoc by Fisher's LSD ${ }^{* * * *} p<0.0001,{ }^{* * *} p<0.001,{ }^{* *} p<0.01,{ }^{*} p<0.05$. Scale bars: $100 \mu \mathrm{m}(\mathrm{A}-\mathrm{C})$; $150 \mu \mathrm{m}(\mathrm{D}, \mathrm{F}-\mathrm{H})$. 

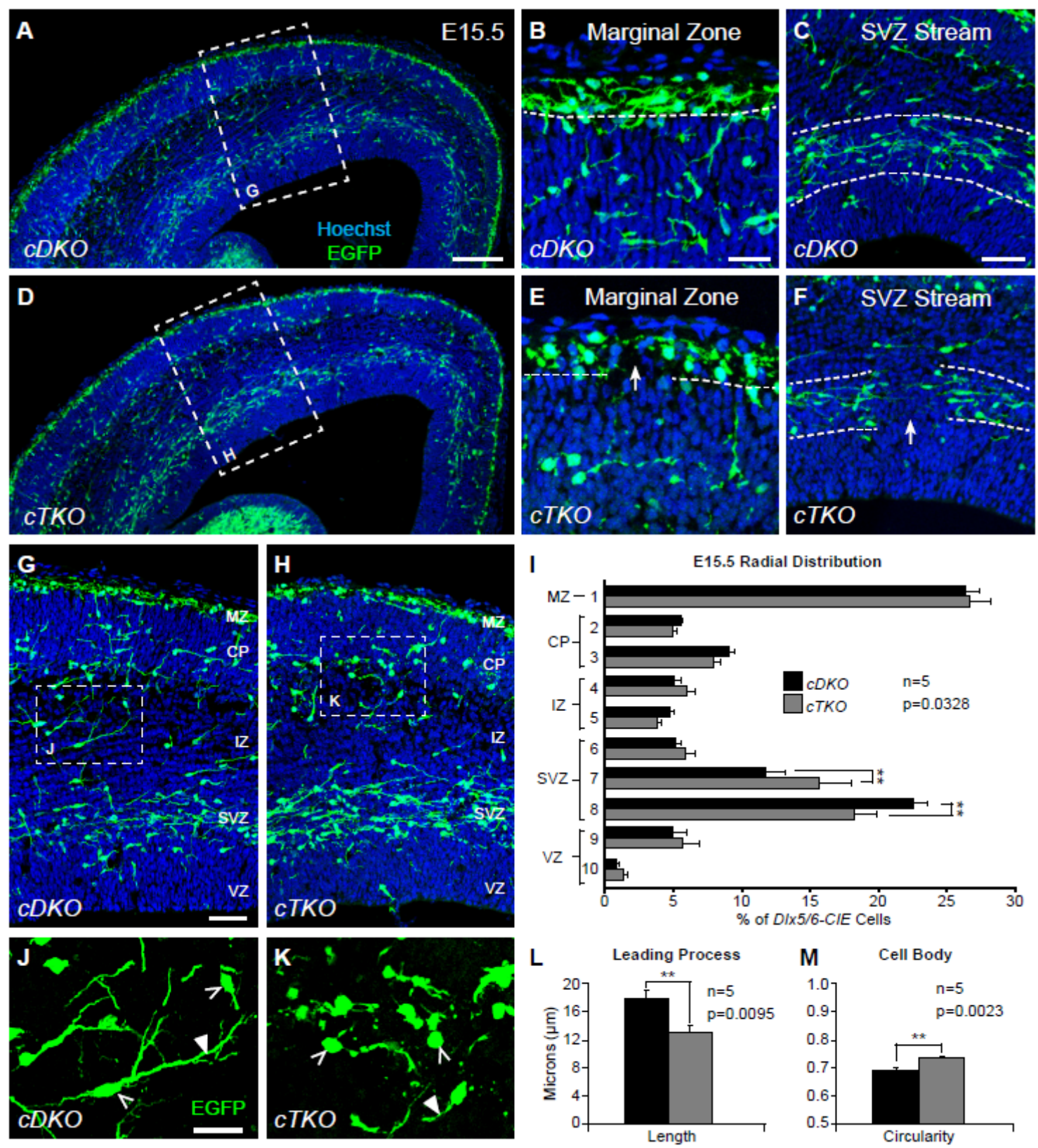

Figure 2.5: Genetic loss of JNK signaling results in interneuron stream displacement and morphological changes at E15.5. (A-C) In conditional double knockout ( $c D K O)$ cortices, interneurons travel in organized streams in the $M Z(B)$ and SVZ (C). (D-F) In conditional triple knockout (cTKO) cortices, frequent gaps are found in the $M Z(E)$ and SVZ (F) streams (arrows). (G-I) Quantification of interneuron distribution in the cortical wall from $n=5$ brains/genotype (Two-way ANOVA: $\left.F_{(9,80)}=2.17 ; p=0.0328\right)$. (J-M) Quantification of interneuron morphology from 
$\mathrm{n}=5$ brains/genotype (Student's $t$-tests). cTKO interneurons (430 cells) have shorter leading processes (closed arrowheads) than cDKO interneurons (510 cells), and cTKO interneurons (656 cells) have more circular cell bodies (open arrowheads) than cDKO interneurons (787 cells). Error bars represent mean \pm s.e.m., post-hoc by Fisher's LSD ${ }^{* *} p<0.01$. Scale bars: 150 $\mu \mathrm{m}(\mathrm{A}, \mathrm{D}) ; 25 \mu \mathrm{m}(\mathrm{B}, \mathrm{E}, \mathrm{J}, \mathrm{K}) ; 50 \mu \mathrm{m}(\mathrm{C}, \mathrm{F}, \mathrm{G}, \mathrm{H})$. 

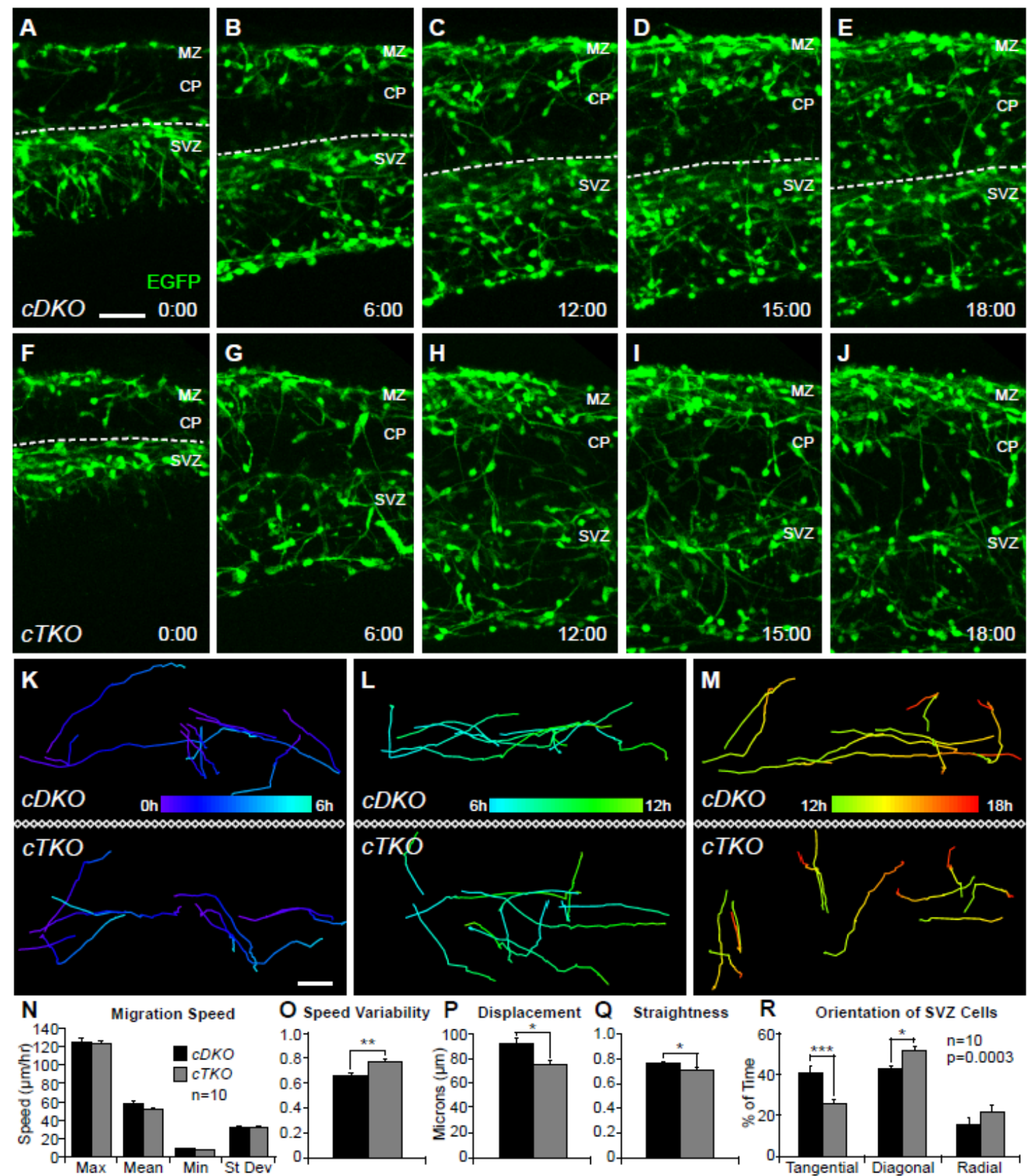

Figure 2.6: Dynamic properties of migrating interneurons are disrupted in ex vivo slices from cTKO brains. (A-E) Movie frames from E14.5 cDKO cortices imaged for 18 hours ex vivo. Dashed line represents the top of the SVZ stream. (F-J) Movie frames from cTKO cortices show premature disbanding of interneurons from migratory streams. (K-M) Representative tracks (pseudo-colored by time) from interneurons recorded during 0-6 hours 
(K), 6-12 hours (L), and 12-18 hours (M) in cDKO and cTKO slices. 10 interneurons were tracked during each time interval for a total of 30 tracks/movie, 300 tracks/genotype. Movies $(n=10)$ collected from 5 different embryos/genotype on 4 experimental days were used for analyses. (N-Q) Quantification of interneuron dynamics during the 12-18 hour timepoint (Student's $t$-tests). (R) Quantification of interneuron leading process orientations (Two-way ANOVA: $\left.F_{(2,54)}=9.63 ; p=0.0003\right)$. Error bars represent mean \pm s.e.m., post-hoc by Fisher's LSD ${ }^{* * *} p<0.001,{ }^{* *} p<0.01,{ }^{*} p<0.05$. Scale bars: $50 \mu \mathrm{m}$. 

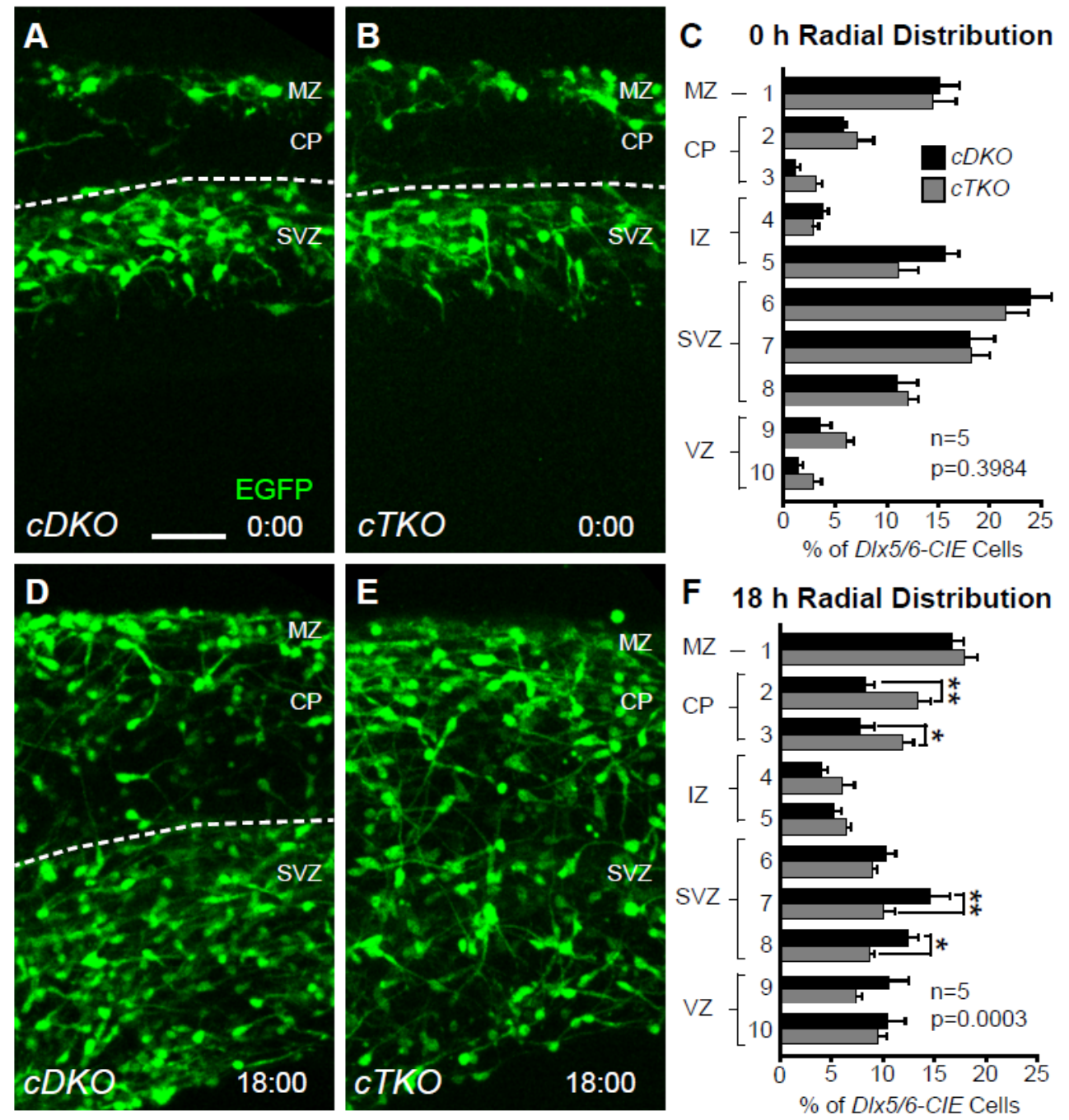

Figure 2.7: Cortical interneurons gradually disperse from migratory streams in cTKO

cortices. (A-B,D-E) Movie frames from 0 and 18-hour timepoints captured from E14.5 cDKO and cTKO ex vivo cortices. (C,F) Quantification ( $\mathrm{n}=5$ brains/genotype) of the radial distribution of interneurons reveals no difference at 0 hours, but significant redistribution by 18 hours (Twoway ANOVA: $\left.F_{(9,80)}=4.06, p=0.0003\right)$. Error bars represent mean \pm s.e.m., post-hoc by Fisher's LSD ${ }^{* *} p<0.01,{ }^{*} p<0.05$. Scale bar: $50 \mu \mathrm{m}$. 

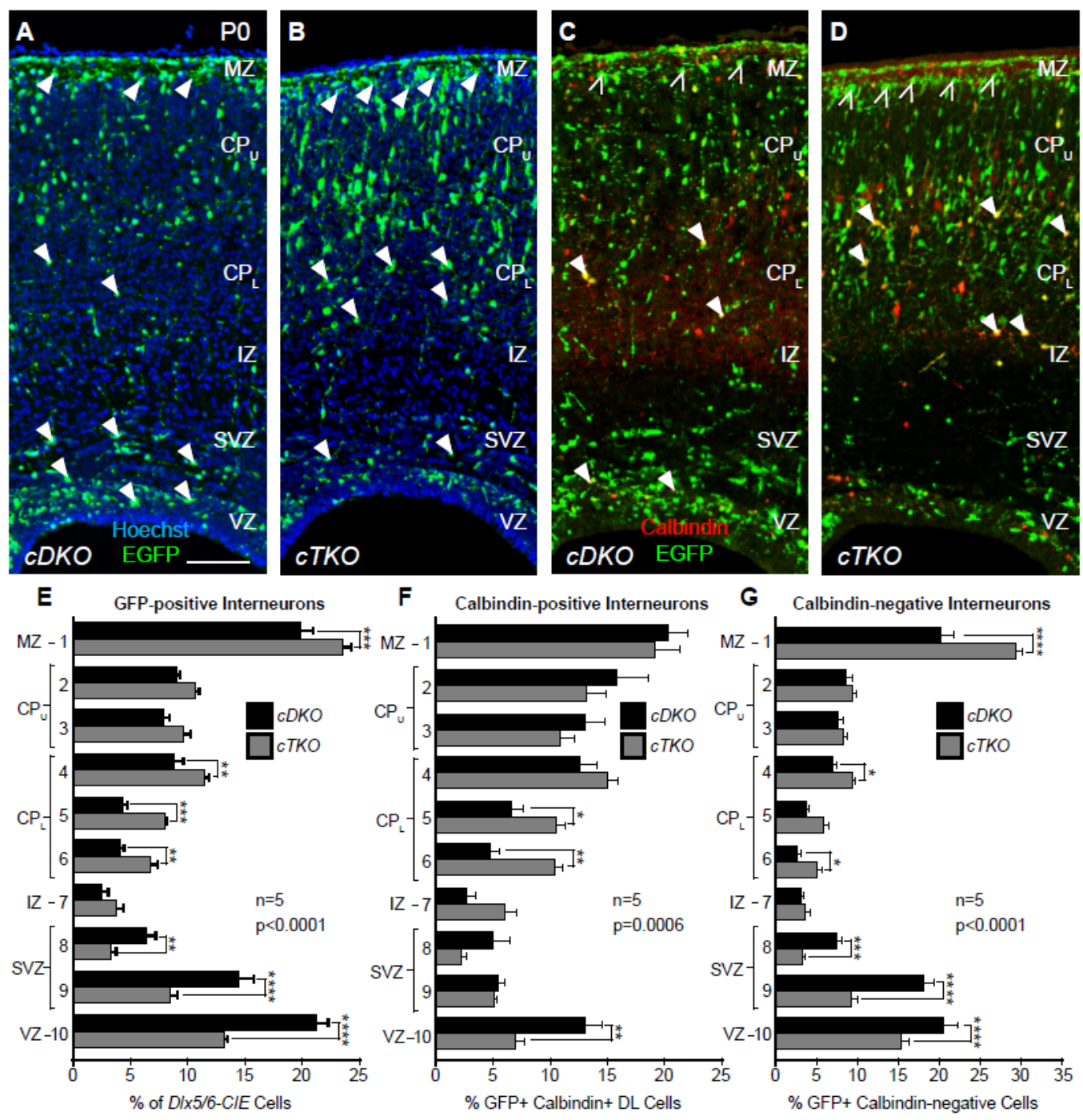

Figure 2.8: Cortical interneurons are mispositioned in the early postnatal cortex of $c T K O$ mice. (A-B) GFP-positive (D/x5/6-CIE) interneurons in the $c D K O(\mathrm{~A})$ and $c T K O(\mathrm{~B})$ cortical wall. (C-D) GFP-calbindin double-labeled (DL) interneurons in the $c D K O(C)$ and $c T K O(D)$ cortical wall. (E-G) Quantification of the radial distribution of GFP-positive (E; Two-way ANOVA: $\left.F_{(9,80)}=20.33, p<0.0001\right)$, DL ( $F$; Two-way ANOVA: $\left.F_{(9,80)}=3.700, p=0.0006\right)$, and GFP-positive calbindin-negative (G; Two-way ANOVA: $F_{(9,80)}=18.77, p<0.0001$ ) interneurons. GFP-positive interneurons are reduced in the VZ/SVZ and are elevated in the lower cortical plate $\left(C_{L}\right)$ and marginal zone (MZ) of the $c T K O$ cortical wall (arrowheads A-B). DL interneurons are reduced in the VZ and elevated in the CPL of the CTKO cortical wall (closed arrowheads, C-D), but 
unchanged in the MZ. GFP-positive calbindin negative interneurons are increased in the $M Z$ (open arrowheads C-D). Quantification from n=5 brains/genotype. Error bars represent mean \pm s.e.m. ${ }^{* * *} p<0.0001,{ }^{* * *} p<0.001,{ }^{* *} p<0.01,{ }^{*} p<0.05$. Scale bar: $100 \mu \mathrm{m}$. 

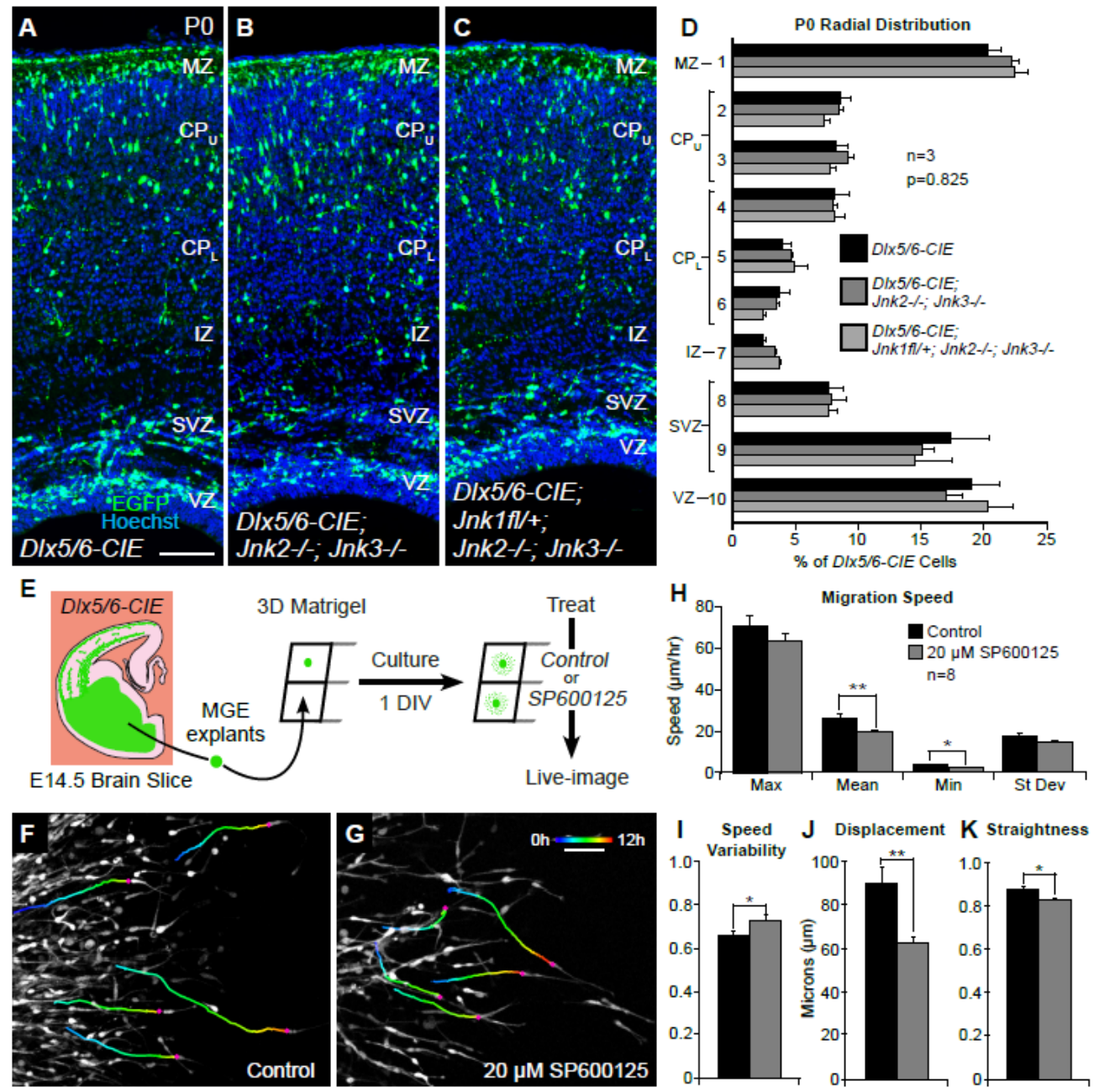

Figure 2.9: Cortical interneurons appear to have a cell-intrinsic requirement for JNK

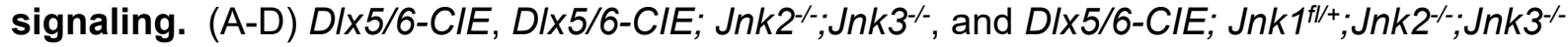

cortices have statistically similar distributions of cortical interneurons, $n=3$ brains/genotype. (E)

E14.5 D/x5/6-CIE medial ganglionic eminence (MGE) explants are grown in a 3D Matrigel assay for 1 day in vitro (DIV), treated with control or $20 \mu \mathrm{M}$ SP600125 media, and then imaged live for 12 hours. (F-G) Tracks (pseudo-colored by time) from 5 representative interneurons in control and $20 \mu \mathrm{M}$ SP600125 conditions. For each condition, 10 interneurons were tracked from $\mathrm{n}=8$ movies (80 tracks/condition) collected from 3 experiments. (H-K) Quantification of dynamic interneuron properties (Student's $t$-tests). Error bars represent mean \pm s.e.m. ${ }^{* *} p<0.01,{ }^{*} p<0.05$. Scale bars: $100 \mu \mathrm{m}(\mathrm{A}-\mathrm{C}) ; 50 \mu \mathrm{m}(\mathrm{F}-\mathrm{G})$. 

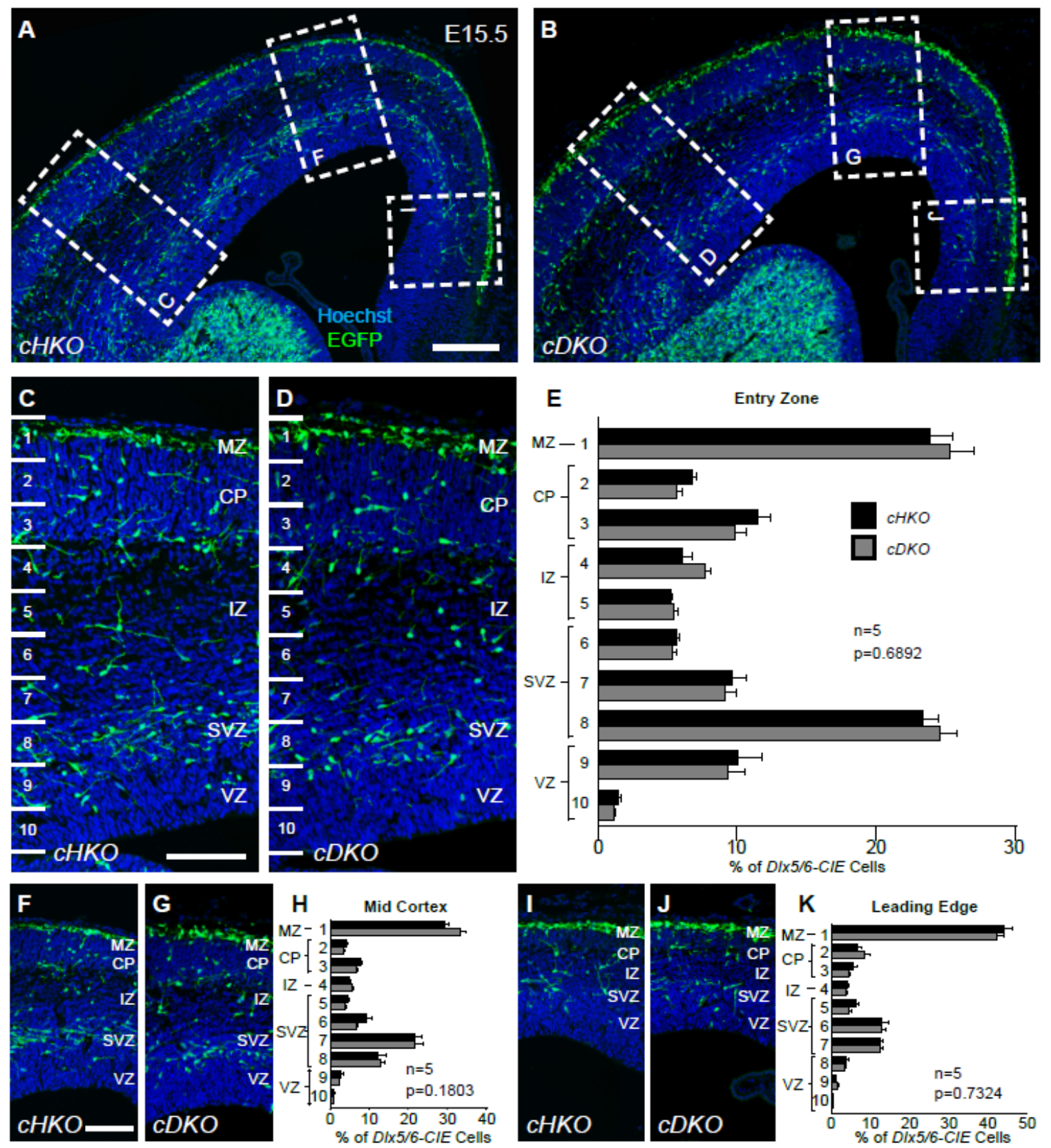

Figure 2.S1: Conditional removal of Jnk1 from Jnk2 knockouts does not alter the radial distribution of cortical interneurons at E15.5 in vivo. (A-B) Interneurons travel in organized streams in $c H K O$ and $c D K O$ cortices at E15.5. (C-D) Binning strategy for interneuron radial distribution. All cropped regions of the cortex were segmented into 10 equidistant bins to determine the radial distribution of cells from the pial to ventricular surface. (C-K) Quantification of the radial distribution of interneurons at lateral (entry zone, C-E), middle (mid-cortex, F-H), 
and medial (leading edge, I-K) locations across the cortical wall revealed no statistically significant differences in the distribution of interneurons between the two genotypes.

Quantification from $n=5$ brains/genotype. Results analyzed by Two-way ANOVA. Error bars represent mean士s.e.m. Scale bars: $200 \mu \mathrm{m}$ (A-B); $100 \mu \mathrm{m}$ (C-D,F-G,I-J). 

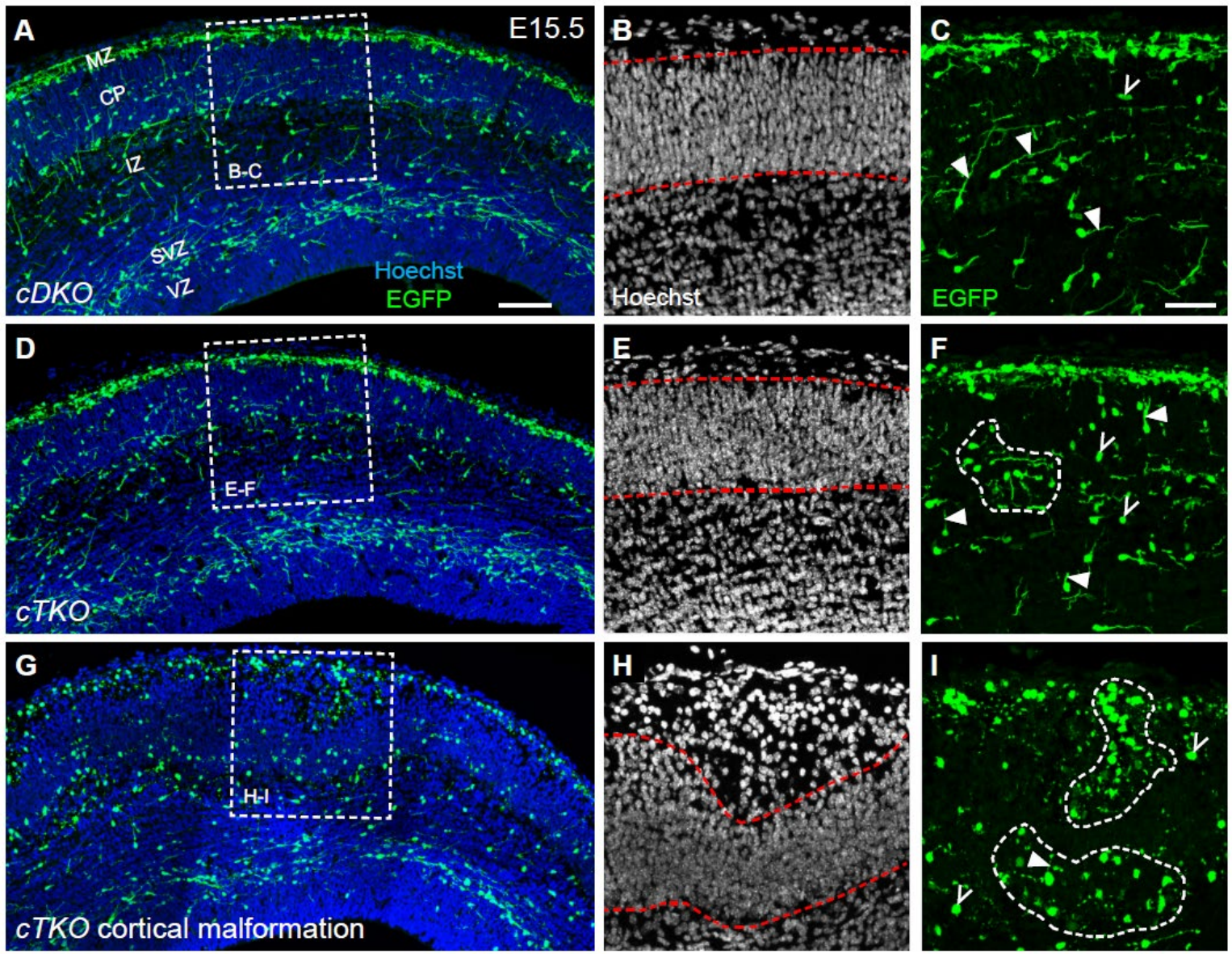

Figure 2.S2: Cortical malformations are present in a subset of $c T K O$ embryos at E15.5.

(A-C) $c D K O$ cortices have an organized $\mathrm{CP}(\mathrm{B}$, outlined in red dashed lines), and migratory

interneurons with long, mainly tangentially-oriented leading processes (C, closed arrowheads)

and elongated cell bodies (C, open arrowhead). (D-F) A representative $c T K O$ brain used for in vivo quantitative analysis of interneuron morphology and distribution (Fig. 2.5) contains an organized cortical plate $(E)$, small clusters of interneurons in the CP region ( $F$, dashed circle), and interneurons with more circular cell bodies ( $F$, open arrowheads) and shorter leading processes (F, closed arrowheads). (G-I) Several cTKO cortices had malformations of the CP at E15.5 $(n=3 ; H)$, which included large CP dips protruding into the $I Z$, and were therefore excluded from quantitative analyses. (I) Interneurons often formed large clusters in and around the malformed regions (dashed circles), and had more pronounced morphological changes to 
cell bodies (open arrowheads) and leading processes (closed arrowheads) than cTKO cortices without severe CP malformations. Scale bars: $100 \mu \mathrm{m}(A, D, G) ; 50 \mu m(B-C, E-F, H-I)$. 


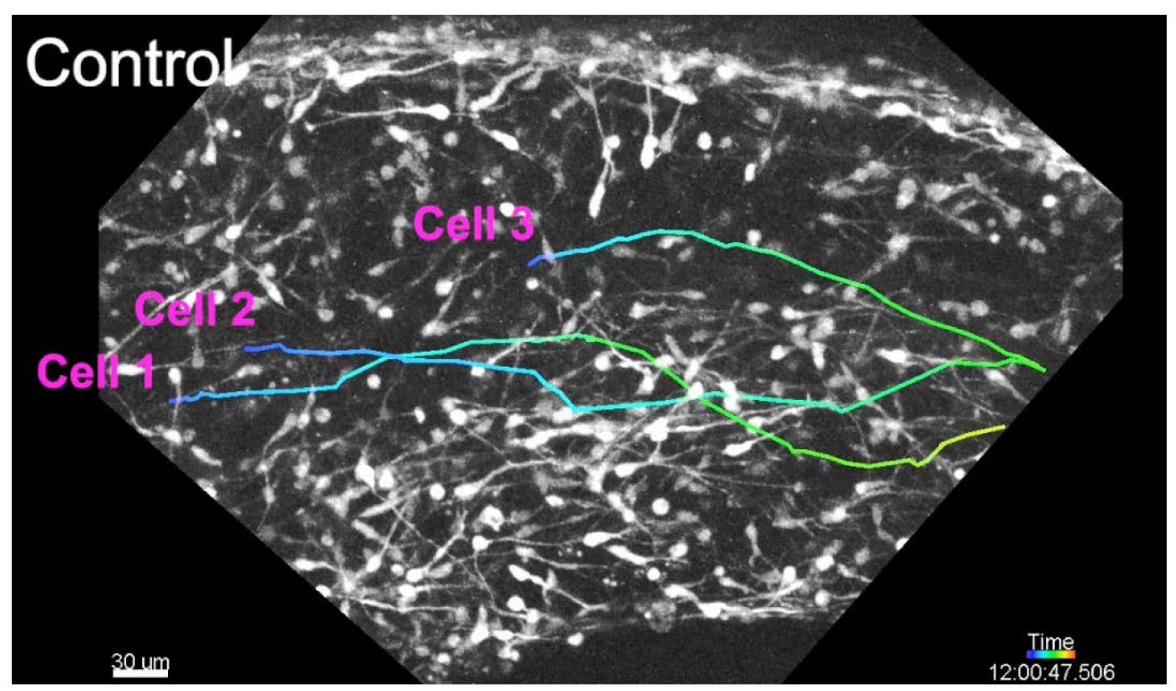

Movie 2.1: Live imaging of migrating cortical interneurons in an E14.5 DIx5/6-CIE brain slice under control conditions. Ventrolateral left, dorsomedial right. Movie Clip 1. Midcortical position of an E14.5 D/x5/6-CIE brain slice imaged for 12 hours in control conditions. Cortical interneurons predominately migrate tangentially in $\mathrm{MZ}$ and SVZ streams as they advance across the cortex. Some cells travel diagonally or radially to switch streams, but rarely stop in the cortical plate region. Movie Clip 2. Three tracked cortical interneurons originating in the SVZ maintain their tangential orientation and remain in the SVZ stream for the duration of the recording. 


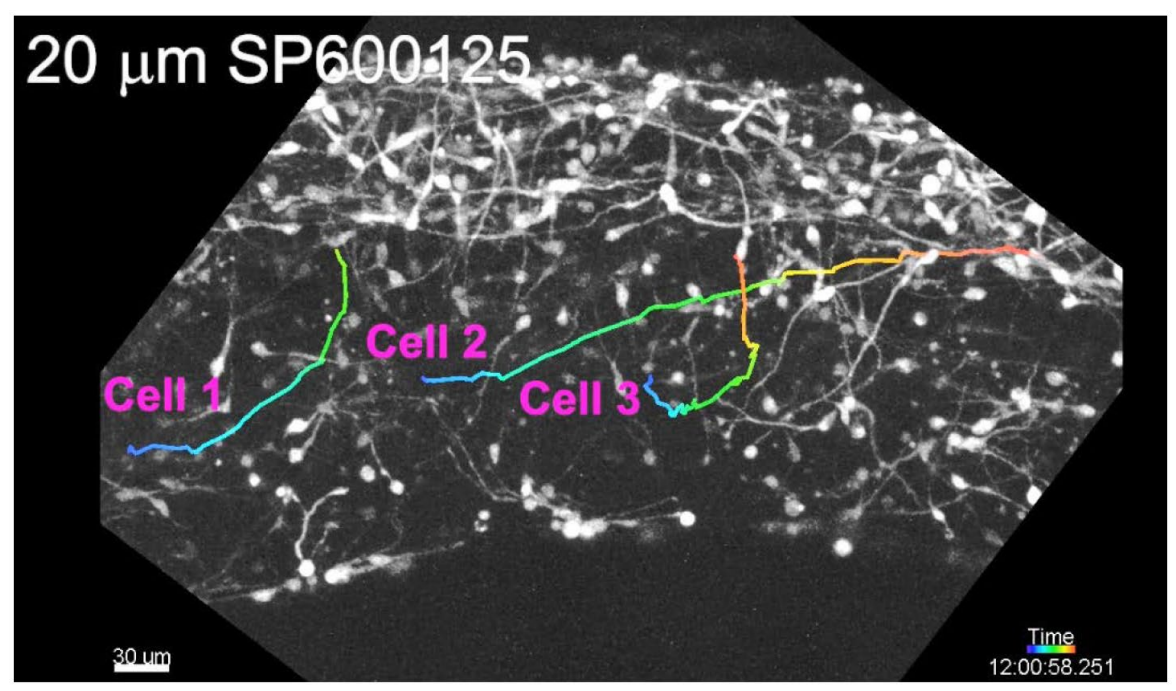

Movie 2.2: Live imaging of migrating cortical interneurons in an E14.5 DIx5/6-CIE brain slice treated with SP600125. Ventrolateral left, dorsomedial right. Movie Clip 1. Mid-cortical position of an E14.5 DIx5/6-CIE brain slice imaged for 12 hours in $20 \mu \mathrm{M}$ SP600125, a pan-JNK inhibitor. Cortical interneurons migrate slower, change trajectories, and evacuate the MZ and SVZ streams to infiltrate the cortical plate by the end of the imaging period. Movie Clip 2. Three tracked cortical interneurons take tortuous trajectories from the SVZ stream to enter the cortical plate, where they remain for the duration of the imaging period. 


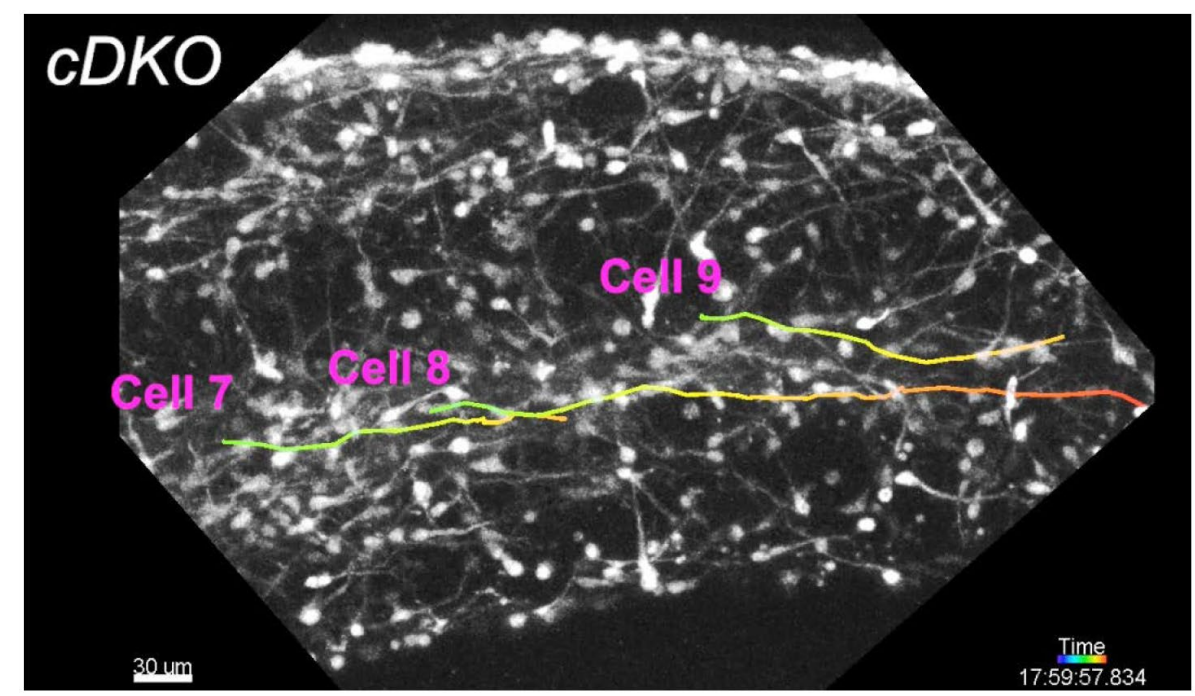

Movie 2.3: Live imaging of migrating cortical interneurons in an E14.5 cDKO brain slice at the mid-cortical position. Ventrolateral left, dorsomedial right. Movie Clip 1. Interneurons in cDKO brain slices migrate in organized $\mathrm{MZ}$ and SVZ streams when imaged ex vivo for 18 hours. Movie Clips 2-4. The 18-hour movies were divided in to three, 6-hour time intervals for quantification of dynamic behavior of interneurons. In $c D K O$ slices, interneurons in all three time intervals maintain a tangential orientation, and migrate in the SVZ throughout the duration of the recording. 


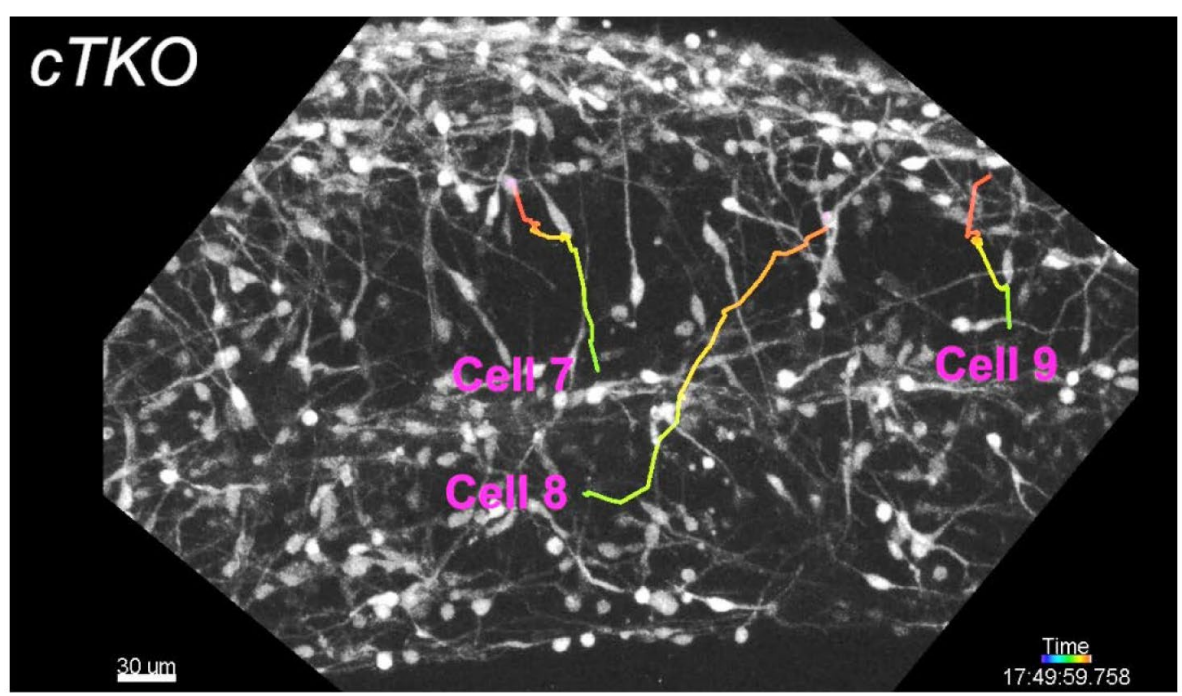

Movie 2.4: Live imaging of migrating cortical interneurons in an E14.5 cTKO brain slice at the mid-cortical position. Ventrolateral left, dorsomedial right. Movie Clip 1. Interneurons in cTKO brain slices begin to migrate in organized streams, but as time progresses, exit streams and infiltrate the cortical plate by the end of the 18-hour imaging period. Movie Clip 2. During the first 6 hours of imaging, some interneurons begin to leave the SVZ (Cell 1), whereas the majority of cells remain in the SVZ (Cells 2, 3). Movie Clip 3. During hours 6-12 of imaging, even more cells begin to evacuate the SVZ (Cells 5,6). Movie Clip 4. During the last 6 hours of imaging, migrating interneurons adopt more convoluted trajectories and infiltrate the cortical plate (Cells 7, 8, 9). 


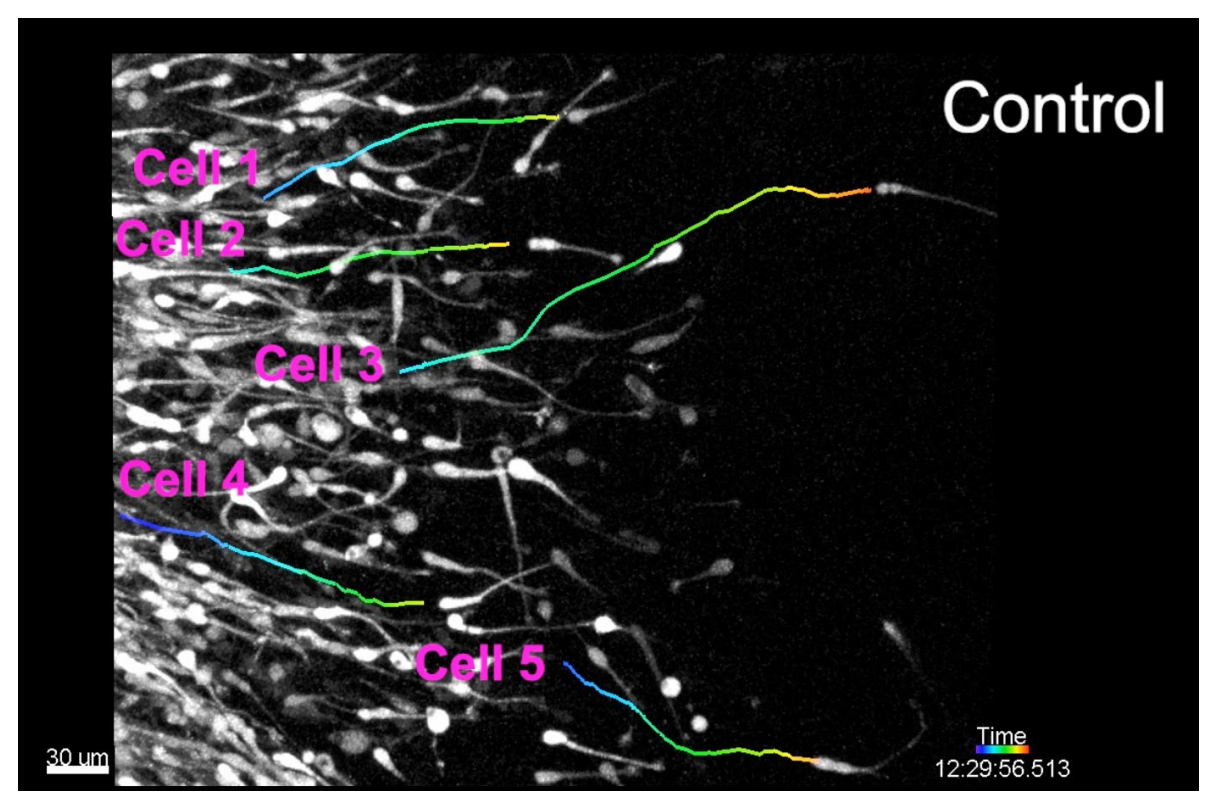

Movie 2.5: Live imaging of migrating MGE cortical interneurons in a 3D Matrigel assay under control conditions. Medial ganglionic eminence (MGE) explant left, outgrowth area right. Interneurons migrating away from E14.5 DIx5/6-CIE MGE explants were imaged for 12 hours in control conditions. Representative tracks from 5 cells demonstrate interneurons migrating in consistent, straight trajectories. 


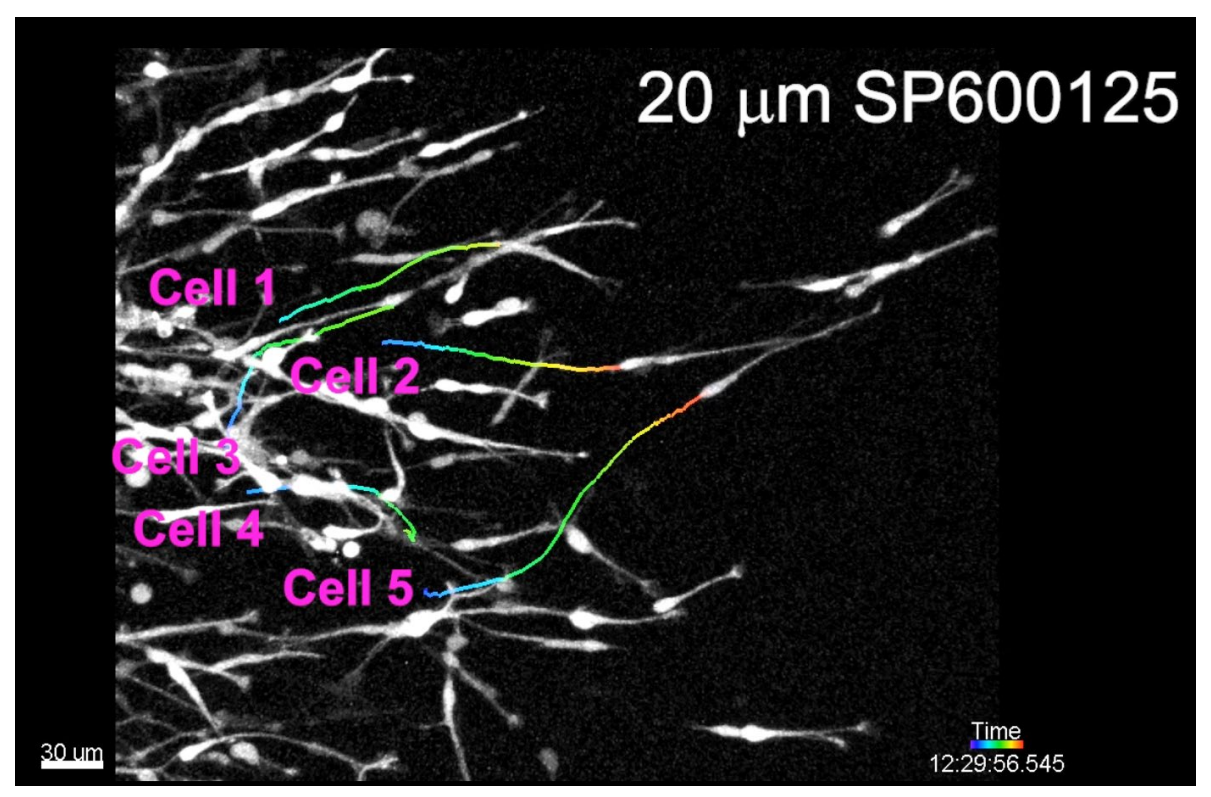

Movie 2.6: Live imaging of migrating MGE cortical interneurons in a 3D Matrigel assay treated with SP600125. Medial ganglionic eminence (MGE) explant left, outgrowth area right. Interneurons migrating away from E14.5 D/x5/6-CIE MGE explants were imaged for 12 hours after $20 \mu \mathrm{M}$ SP600125 treatment. Representative tracks from 5 cells show interneurons migrating slower in shorter, less straight trajectories. 


\section{Chapter 3: A non-autonomous requirement for JNK signaling in early thalamocortical axon pathfinding}

Jessica G. Cunningham ${ }^{1,2,3}$, James D. Scripter ${ }^{1,2,3}$, Stephany A. Nti ${ }^{1,3}$, Eric S. Tucker ${ }^{1,3}$

${ }^{1}$ Department of Neuroscience, ${ }^{2}$ Neuroscience Graduate Program, ${ }^{3}$ Rockefeller Neuroscience Institute, West Virginia University School of Medicine, Morgantown, WV 26506 


\begin{abstract}
Thalamocortical connectivity is essential for proper sensory processing. Axonal pathways between the thalamus and cortex are established early in development, and require the guidance of intermediate guidepost cells and axons to correctly navigate the developing forebrain. In this study, we identify a novel role for the c-Jun N-terminal Kinase (JNK) signaling pathway in thalamocortical axon guidance. In a mouse model with loss of JNK from the DIx5/6 territory, thalamocortical axons aberrantly project ventrally from the thalamus and into the hypothalamus, and are unable to cross the diencephalon-telencephalon boundary (DTB). In addition, corticothalamic, striatonigral, and nigrostriatal axons are all unable to cross the DTB and fail to reach their intended targets. The telencephalic corridor is morphologically altered, and guidepost cells in the ventral telencephalon are severely reduced. This study is the first to define an essential, non-autonomous requirement for JNK signaling in the early establishment of thalamocortical connectivity.
\end{abstract}




\section{Introduction}

The thalamus relays essential sensory processing information to the cerebral cortex via thalamocortical axons (Jones, 2001). The thalamocortical axon pathway is a longitudinal axon projection in the forebrain, and must extend over a long distance to reach precisely defined targets in the cortex (Leyva-Diaz and Lopez-Bendito, 2013). Throughout development, thalamocortical axons use a combination of long- and short-range guidance cues (Braisted et al., 1999), and rely on intermediate targets and guideposts to guide their trajectories (Squarzoni et al., 2015). Disruptions to thalamocortical connectivity, even from early in neural development, can lead to disorders such as autism, schizophrenia, and bipolar disorder (Anticevic et al., 2014; Nair et al., 2013).

The formation of the thalamocortical pathway occurs throughout embryonic and early postnatal development in the mouse brain. Thalamocortical axons must first extend ventrally from the dorsal thalamus, traverse through the prethalamus, then cross the diencephalontelencephalon boundary (DTB) around embryonic (E) day 12.5 (Lopez-Bendito and Molnar, 2003). Once in the ventral telencephalon, thalamocortical axons extend through the developing internal capsule, guided by gradients of axon guidance cues such as Netrins, Slits, Ephrins, and Semaphorins expressed in the developing striatum (Molnar et al., 2012). At the pallial-subpallial boundary, thalamocortical axons meet descending corticothalamic axons (Molnar et al., 1998; Molnar and Blakemore, 1995), before coursing into the cortex to their defined targets. The correct patterning and cellular composition of the expansive territory through which thalamocortical axons must traverse is critical for establishing proper connectivity.

In addition to establishing gradients for the proper sorting of thalamocortical axons, the ventral telencephalon is a critical intermediate target for thalamocortical axon pathfinding, containing both corridor and guidepost cells that are necessary for proper guidance of thalamocortical axons in early development. Corridor cells migrate from the lateral ganglionic eminence and form a growth-permissive territory for thalamocortical axons between the medial 
ganglionic eminence and the nascent globus pallidus (Bielle et al., 2011a; Lopez-Bendito et al., 2006). Guidepost cells reside in the ventral telencephalon and send axons back to the thalamus and prethalamus, and are thought to provide scaffolding for thalamocortical axons (Braisted et al., 1999; Metin and Godement, 1996; Molnar and Cordery, 1999). Disruptions to both of these territories have been described in mutants with thalamocortical axon pathfinding phenotypes, notably in the ability of thalamocortical axons to cross the DTB (Lopez-Bendito, 2018; Molnar et al., 2012). Indeed, disruptions to this critical intermediate territory have been shown to non-autonomously affect proper thalamocortical axon guidance, as these mutations are not targeting the thalamocortical axons themselves.

The DIx (distal-less) homeobox genes are arranged as three bigene clusters $(D / x 1 / 2$, $D / x 3 / 4$, and $D / \times 5 / 6)$ in mice, and encode a family of transcription factors that are critical for the development of GABAergic interneurons (Zerucha and Ekker, 2000). The D/x5/6 domain is expressed in the developing forebrain (Panganiban and Rubenstein, 2002), notably spanning the territory that encompasses both corridor and guidepost cells. The intergenic region of the DIx5/6 bigene cluster contains the cis-acting regulatory element I56ii which is essential for the development of corridor cells (Fazel Darbandi et al., 2016). Interestingly, in mice with conditional deletions of Linx (Mandai et al., 2014), Celsr3 (Zhou et al., 2008; Zhou et al., 2009), or Frizzled3 (Hua et al., 2014; Qu et al., 2014) in the D/x5/6 region, thalamocortical axons are non-autonomously misrouted. This implicates the $D / x 5 / 6$ territory as a critical intermediate region for the extension of thalamocortical axons.

Disruptions to intermediate guideposts could non-autonomously influence axons in a variety of ways. Signaling gradients could be disrupted, which would impact the normal attractive and repulsive signals in the developing striatum. Additionally, the generation, migration, or axon extension of intermediate targets, such as the corridor or guidepost cells, could also be affected, resulting in disruptions to thalamocortical axons. c-Jun N-terminal Kinase (JNK) is a mitogen-activated protein kinase which has been shown to play a role in 
various aspects of neural development, including cellular migration and axon extension (Coffey, 2014). We therefore hypothesized that JNK function in the ventral telencephalon could play a role in early thalamocortical axon guidance.

Here, we show that complete loss of JNK function from the $D / \times 5 / 6$ territory results in thalamocortical axon misrouting from early in development. Thalamocortical axons are unable to cross the diencephalon-telencephalon boundary (DTB), and instead misroute both ventrally into the hypothalamus, and rostrally along the DTB. Telencephalic corridor cells are mispositioned, and guidepost cells are significantly reduced in number. Additionally, corticothalamic, striatonigral, and nigrostriatal axons are unable to project across the DTB and thus fail to reach their targets. This is the first time that JNK signaling has been shown to have a non-autonomous requirement in thalamocortical axon pathfinding. 


\section{Results}

\section{Axon tracts are missing or misrouted in JNK conditional triple knockout (cTKO) mice}

Longitudinal axon tracts in the developing forebrain use intermediate targets as guideposts during their extensive trajectories to their targeted regions. The ventral telencephalon encompasses the developing internal capsule, which serves as an intermediate target for both thalamocortical and corticothalamic axons. To determine the requirement for JNK signaling in this critical intermediate territory, we developed a conditional triple knockout (cTKO) mouse that conditionally removes Jnk1 from DIx5/6-CIE lineage cells of constitutive Jnk2;Jnk3 double knockouts. This effectively removes all of JNK signaling from the territory through which major axon pathways traverse in early development.

We began by labeling embryonic (E) day 17.5 brain sections with $L 1$, a transmembrane adhesion molecule that labels many axon tracts in the developing brain (Stallcup et al., 1985). In control brains at this age, axons span the internal capsule region, connecting the cortex to the thalamus (Fig. 3.1 A,C, n=3/3). In cTKO mice however, the internal capsule was completely missing (Fig. 3.1 B,D, n=3/3), and there were no connections between the diencephalon and telencephalon. Additionally, a large, "U-shaped" bundle of axons was present underneath the hypothalamus (Fig. 3.1 B, $n=3 / 3$ ). Commissural axon tracts including the anterior commissure and corpus callosum were present in the cTKO brain and still crossed the midline, but appeared to be reduced in size and trajectory (Fig. 3.S1 A-D).

Axons were present in the cortex of cTKO mice, however also appeared reduced in number. To determine the identity of the cortical axons, we labeled E15.5 brain sections with L1 and Tag1, a neural adhesion molecule which labels corticofugal axons (Wolfer et al., 1994) (Fig. 3.1 E-L). L1-positive and Tag1-positive axons were present in the intermediate zone (IZ) of both the control (Fig. 3.1 E-H; n=7/7) and cTKO (Fig. 3.1 I-L; n=7/7) cortical walls at E15.5. 
However, L1-positive/Tag1-negative fibers, which travel in the upper IZ, were completely absent from cTKO brains (Fig. 3.1 I-L, n=7/7), suggesting that a population of axons which originated sub-cortically was affected. Additionally, Calbindin-labeled axons were completely absent from cTKO brains at E15.5 (Fig. 3.S1 E-F, n=11/11). Thus, we found several major axon deficiencies in $c T K O$ forebrains, including a loss of axons normally traversing the upper IZ in the cortical wall, a disrupted internal capsule, and an ectopic accumulation of axonal fibers coursing beneath the hypothalamus.

\section{Thalamocortical axons misroute ventrally in cTKO mice}

Since L1-postive/Tag-1 negative axons were absent from cTKO cortices, and there was an ectopic L1-positive bundle of axons beneath the thalamus, we examined whether thalamocortical axons were aberrantly projecting in cTKO brains. We placed a Dil crystal into the dorsal thalamus of fixed brain slices at E15.5 to observe the projection pattern of thalamocortical axons (Fig. 3.2 A-B). In control slices, Dil-labeled axons extended from the thalamus, crossed the diencephalon-telencephalon boundary (DTB), and projected through the internal capsule in the telencephalon (Fig. 3.2 C, n=8/8). However, in cTKO slices, Dil-labeled axons projected to the DTB, but rather than crossing into the telencephalon at this level, turned ventrally and coursed through the hypothalamus (Fig. 3.2 D, n=3/3).

To further assess the trajectory of thalamocortical axons, we labeled E15.5 brain sections with NetrinG1, a netrin that is enriched in the dorsal thalamus during development, and serves as a marker of most thalamocortical axons (Nakashiba et al., 2000). NetrinG1 is distinct from the axon guidance molecule Netrin1. In control brains, NetrinG1-positive thalamocortical axons crossed the DTB at caudal levels, extended through the internal capsule, and reached the mid-cortical wall (Fig. 3.2 E-I, n=4/4). However, in cTKO brains, we observed two different patterns of thalamocortical axon misrouting. At caudal levels, thalamocortical axons extended 
from the thalamus, but instead of crossing the DTB, projected ventrally into the hypothalamus in all cTKO brains examined (Fig. 3.2 L-N, n=15/15). Additionally, a second bundle of thalamocortical axons extended rostrally in a large fascicle along the DTB (Fig. 3.2 J-L, $\mathrm{n}=15 / 15)$. From this rostrally-projecting bundle, some thalamocortical axon fibers splayed into the ventral forebrain at ectopic positions (Fig. 3.2 J-K), but with varying degrees of penetration (Fig. 3.S2). At E15.5, we observed cTKO brains with no axons present in the telencephalon (Fig. 3.S2 C-D, n=6/15), axons entering the telencephalon but not reaching the internal capsule (Fig. 3.S2 E-F, n=5/15), axons ectopically reaching the internal capsule but not the cortical rudiment (Fig. 3.S2 G-H, n=2/15), and axons reaching the cortical rudiment but not in the correct number or location (Fig. 3.S2 I-J, n=2/15). Together, both Dil and immunohistochemical analyses indicate that thalamocortical axons are massively misrouted at the DTB region in cTKO brains.

\section{Thalamocortical and corticothalamic axons take aberrant trajectories in cTKO mice}

Immunohistochemical analysis revealed that thalamocortical axons mis-projected both ventrally and rostrally in cTKO brains at E15.5. Despite failing to cross the DTB and properly extend through the internal capsule, some thalamic projections entered the telencephalon at aberrant locations (Fig. 3.2S, n=9/15). To determine whether these fibers eventually extended into the cortex, we examined brains at postnatal $(P)$ day 0 , the oldest age we were able to collect viable cTKO mice (Table 3.1). PO brains were collected, fixed, hemisected along the midsagittal plane, and a Dil crystal was placed into the dorsal thalamus (Fig. 3.3A).

In control brains, Dil-labeled axons projected from the thalamus, across the DTB, through the internal capsule, and into the cortex (Fig. 3.3 B-E; $n=5 / 5$ ). However, in all cTKO brains examined, thalamocortical axons misrouted ventrally into the hypothalamus (Fig. 3.3 H-I, 
L-M, n=4/4), verifying our NetrinG1 immunohistochemistry (Fig. 3.2 M) and Dil-labeled slice (Fig. 3.2D) results at E15.5. The axons that did not extend into the hypothalamus instead projected rostrally on the ventral surface of the brain near the DTB (Fig. 3.3 F-G, J-K). Similar to the E15.5 timepoint, thalamocortical axons were observed invading the striatum from an ectopic ventral position (Fig. 3.3 F-G, J-K). These ectopically projecting Dil-labeled fibers only reached the cortex in half of the cTKO brains examined (Fig. $3.3 \mathrm{~J}, \mathrm{n}=2 / 4$ ), and when they did, they were less abundant and less advanced than their control counterparts. We also noticed increased ventricular volume in cTKO slices, which has been shown in other mutants with thalamocortical axon guidance phenotypes (Hua et al., 2015; Hua et al., 2014; Tissir et al., 2005; Wang et al., 2002). CT scans were performed on whole heads at P0, where we observed increased ventricular volume in the intact $c$ TKO brain (Fig. 3.3, n=6/6).

Since thalamocortical and corticothalamic axons have been shown to be intimately related with one another during development, we asked whether the subcortical trajectory of cortical axons was also compromised in cTKO mice. Using the same experimental design as above (Fig. 3.3A), we placed a crystal of Dil into the cortex of the other half of the P0 brains (Fig. 3.4A). In control brains, Dil-labeled corticothalamic axons assumed a normal trajectory through the internal capsule, crossed into the diencephalon, and reached the dorsal thalamus (Fig. 3.4 B-D, n=5/5). Additionally, Dil-labeled cell bodies were present in the thalamus from retrograde labeling of thalamocortical axons (Fig. 3.4 D-E, $n=5 / 5$ ). In cTKO brains however, cortical axons were severely misrouted and did not coalesce into an organized internal capsule (Fig. 3.4 F-G,H-K, n=4/4). Instead, cortical axons ectopically entered the subpallium in large, disorganized fascicles at rostral levels (Fig. 3.4 F,J-K, n=4/4). Cortical fibers only reached the thalamus in half of the cTKO brains examined (Fig. 3.4 L, n=2/4). Interestingly, backfilled thalamic cells were only found in CTKO brains that had corticothalamic fibers in the thalamus, and when present, were reduced in number (Fig. 3.4 L-M). 
We observed a range of axon misrouting phenotypes in cTKO mice in both thalamic and cortical Dil experiments at P0. Since a Dil crystal was placed into either the thalamus (Fig. 3.3) or cortex (Fig. 3.4) of the same hemisected brain, we compared the degree of axon misrouting between the thalamic and cortical Dil placements. Indeed, we found that in the cTKO brains that lacked thalamic innervation of the cortex (Fig. 3.3 F-I), no cortical axons had reached the thalamus (Fig. 3.4 F-G). Additionally, no backfilled thalamic cells were present after the cortical Dil placement (Fig. 3.4 H-I), indicating that thalamic axons had not reached the cortex in both halves of each brain examined. Similarly, in cTKO brains with partial thalamic innervation of the cortex (Fig. 3.3 J-M), some cortical axons had reached the thalamus (Fig. 3.4 J-L), and backfilled thalamic cells were present (Fig. 3.4 L-M). Thus, reciprocal thalamocortical and corticothalamic projections were comparably affected in each of the cTKO brains examined.

\section{Thalamocortical axons are misrouted early in development in cTKO brains}

We next assessed the developmental time course of thalamocortical axon misrouting in cTKO brains. Using NetrinG1 as a marker for thalamocortical axons, we examined brains ranging from E12.5 to P0 (Fig. 3.5 A-J). At E12.5, when thalamocortical axons first cross from the diencephalon into the telencephalon (Fig. 3.5 A inset, n=2/2), no NetrinG1-positive fibers crossed the DTB in cTKO mice (Fig. $3.5 \mathrm{~F}$ inset; $\mathrm{n}=2 / 2$ ). Similarly, at $\mathrm{E} 13.5$ when the furthest advanced thalamocortical fibers have approached the pallial-subpallial boundary (Fig. 3.5 B, $\mathrm{n}=6 / 6$ ), NetrinG1-positive axons were absent from the ventral telencephalon in cTKO mice (Fig. 3.5 G, n=8/8). When we examined the diencephalon-telencephalon boundary region at $\mathrm{E} 13.5$, where NetrinG1-positive axons normally cross the DTB in control brains (Fig. 3.5 K-L, n=6/6), we observed ventrally projecting thalamocortical axons in cTKO brains that failed to cross the DTB (Fig. 3.5 M-N, n=8/8). Thus, the misrouting of thalamocortical axons begins in early development, when thalamocortical axons would normally first be crossing the DTB region. 
Thalamocortical axons were similarly misrouted in cTKO brains at E15.5 (Fig. $3.5 \mathrm{H}, \mathrm{n}=15 / 15)$, E17.5 (Fig. $3.5 \mathrm{I}, \mathrm{n}=3 / 3$ ), and P0 (Fig. $3.5 \mathrm{~J}, \mathrm{n}=5 / 5$ ), with the size of the "U-shaped" bundle increasing in thickness as development proceeds.

In E13.5 cTKO brains, thalamocortical axons extended ventrally from the thalamus and through the prethalamus (Fig. 3.5 M-N, n=8/8), suggesting their initial extension in the diencephalon is grossly unaffected. However, after exiting the prethalamus, thalamocortical axons were unable to cross the DTB. Interestingly, this pathway is populated by $D / x 5 / 6-C / E-$ positive cells and axons, which form a diencephalic corridor from the prethalamus to the ventral forebrain in control brains (Fig. 3.5K-L). In cTKO brains however, DIx5/6-CIE-positive cells were present either in the telencephalon or diencephalon, but like the thalamocortical axons, failed to span the DTB (Fig. 3.5 M-N). Indeed, D/x5/6-CIE-positive cells appeared to form a piled-up wedge on the diencephalic side of the DTB (Fig. 3.5 M-N). These data collectively suggest that thalamocortical axons are able to extend properly from the thalamus to the DTB in cTKO brains, however at the DTB region, there is a disruption to both thalamocortical axons and D/x5/6-CIEpositive cells and axons, which are the target of our conditional JNK deletion.

\section{Telencephalic corridor and guidepost cells are disrupted in cTKO mice}

The corridor is a permissive territory in the developing ventral telencephalon that supports the extension of thalamocortical axons through an otherwise growth inhibitory environment (Lopez-Bendito et al., 2006). Since disruptions to the corridor region have been implicated in thalamocortical axon misrouting, and the $D / x 5 / 6$ domain spans this region, we examined the corridor in control $(n=4)$ and $c T K O(n=5)$ brains at $E 13.5$ at 4 different rostrocaudal locations (Fig. 6). Brain slices were labeled with Islet1 to reveal corridor cells (Fig. 3.6 BE), which migrate ventral-medially from the lateral ganglionic eminence (LGE) to position themselves between the Nkx2.1-positive medial ganglionic eminence (MGE) progenitor domain 
and the nascent globus pallidus (GP). The Islet1-positive corridor extends caudally to the DTB, where thalamocortical axons project from the diencephalon into the telencephalon (Fig. 3.6 DE). In CTKO brains, the distribution of Islet1-positive cells is similar to control brains at rostral levels (Fig. 3.6 F). However, immediately caudal to this location, Islet1-positive cells are ectopically located in a region corresponding to the globus pallidus in control brains (Fig 3.6 G). At more caudal levels, where Islet1-positive cells normally associate with thalamocortical axons in control brains (Fig. 3.6 D-E), the Islet1-positive cells are severely reduced in number in cTKO brains, and no thalamocortical axons have crossed the DTB (Fig. 3.6 H-I).

Additionally, the Nkx2.1 expression domain is altered in cTKO mice. In control brains, Nkx2.1-positive GP cells are present throughout the corridor region and reside directly beneath the thalamocortical axons as they enter the telencephalon (Fig. 3.6 D-E). However, the GP was irregularly shaped in $c T K O$ brains, with Nkx2.1-positive cells spreading out to the lateral, pial margin of the ventral telencephalon (Fig. 3.6 F). Slightly more caudal, the Nkx2.1-positive GP shifted dorsal and lateral, in some cases splitting with the apparent influx of ectopically positioned Islet1-positive cells (Fig. 3.6 G). Similar to Islet1-positive cells, Nkx2.1-positive cells are missing from the caudal corridor of $c T K O$ brains, where thalamocortical axons should cross into the telencephalon (Fig. 3.6 H-I). In control brains, both Islet1- and Nkx2.1-positive cells are precisely positioned at the point of axon crossing at the DTB (Fig. 3.6 E,K), however, these two populations of cells fail to extend caudally to the DTB in cTKO brains (Fig. 3.6 I,L).

In control brains, a population of diencephalic Islet1-positive cells form a scaffold beneath the thalamocortical axons (Fig. 3.6 E,K). Unlike telencephalic Islet1-positive cells that nearly disappeared at this level in cTKO brains, diencephalic Islet1-positive cells were present in seemingly normal numbers in cTKO mice (Fig. 3.6 I,L). However, these Islet1-positive cells stopped short of the DTB at the position where thalamocortical axons misrouted ventrally (Fig. 3.6 I,L). These data suggest that at the level of the DTB, both Islet1- and Nkx2.1-positive cells 
are mispositioned or even missing from the ventral telencephalon, which may account for the inability of thalamocortical axons to cross the border.

Another population of cells thought to assist thalamocortical axons across the DTB are guidepost cells (Braisted et al., 1999), which reside in the ventral telencephalon and extend axons to the dorsal thalamus. Since these cells are often missing or reduced in number in mutants with thalamocortical axon defects, we investigated whether they were present in the brains of cTKO mice. While the molecular profile of these guidepost cells remains unknown, they can be retrogradely labeled by placing a Dil crystal into the dorsal thalamus (Fig. 3.7). In E13.5 control brains, a bundle of thalamocortical axons crossed the DTB and projected through the ventral telencephalon (Fig. 3.7 A-D; n=6/6). Retrogradely-labeled cells were found in close association with the internal capsule at both rostral and caudal levels of the ventral telencephalon in territories that were DIx5/6-CIE positive (Fig. 3.7 A-D). However, in cTKO brains, thalamocortical axons projected ventrally from the thalamus and did not cross the DTB (Fig. 3.7 E-H; n=6/6). At rostral levels, there were no labeled axons in the telencephalon (Fig. 3.7 E-F). Although a few scattered retrogradely-labeled cells were labeled, they were reduced in number (Fig. 3.7 E-H) when compared to control brains. Collectively, the disruption to the telencephalic corridor and the reduced number of guidepost cells indicate that the territory through which thalamocortical axons normally traverse is altered in cTKO brains.

\section{Reciprocal connections between the striatum and substantia nigra are altered in cTKO mice}

Besides thalamocortical and corticothalamic axons, several other axonal pathways cross the DTB during early development. Two pathways in particular reciprocally connect the striatum to the substantia nigra (striatonigral) and the substantia nigra to the striatum (nigrostriatal). Since these pathways course in close proximity to thalamocortical axons as they approach the 
DTB, and disruptions to both the striatonigral and nigrostriatal pathways have been observed in mice with thalamocortical axon guidance defects, we examined striatal and nigral axon projection patterns in cTKO mice. In control brains, Darpp32-positive striatal axons project from the striatum, across the DTB, and reach the substantia nigra in the midbrain (Fig. 3.8 A,C-E,I $n=6 / 6)$. Reciprocally, tyrosine hydroxylase $(\mathrm{TH})$-positive nigral axons project from the substantia nigra to join the median forebrain bundle, cross the DTB, and disperse diffusely in the striatum (Fig. 3.8 A,I n=6/6). However, in cTKO brains, Darpp32-positive fibers coalesce and are entangled in the striatum (Fig. 3.8 B,F; $n=5 / 5$ ). These striatal fibers do not cross the DTB and are therefore missing at the level of the substantia nigra (Fig. 3.8 B, G-H,J). Nigrostriatal fibers are likewise unable to fully extend along the anterior-posterior axis in cTKO brains (Fig. 3.8 B), and instead misroute in a "U-shaped" bundle into the hypothalamus (Fig. 3.8 $\mathrm{J}, \mathrm{n}=8 / 8$ ), similar to the NetrinG1-positive thalamocortical axons. In both the thalamocortical and nigrostriatal pathways, we observe similar patterns of misrouting, in which normally ipsilaterally projecting tracts are misrouted and become commissural. We next wanted to determine if nigrostriatal axons mis-projected early in development similar to thalamocortical axons. Indeed, TH-positive axons misrouted as early as E13.5 ( $n=4 / 4)$, and had formed a similar "U-shaped" bundle by E15.5 (Fig. 3.S4; n=15/15). In the cTKO model, multiple longitudinal axon pathways are unable to properly extend along the rostrocaudal axis, fail to cross the DTB, and some incorrectly project across the midline.

\section{Non-autonomous requirement for JNK signaling in thalamocortical axon}

\section{pathfinding}

Although our data strongly implicate a non-autonomous role for JNK signaling in establishing major forebrain axon tracts, we cannot exclude the possibility that the constitutive loss of Jnk2 and Jnk3 in the cTKO model impacts thalamocortical projections. In the cTKO 
model, Jnk1 is conditionally removed from DIx5/6-CIE cells in a Jnk2;Jnk3 knockout mouse (Fig. 3.9 B), where thalamocortical axons themselves have lost Jnk2 and Jnk3 function. To test whether the loss of Jnk2 and Jnk3 alone impairs thalamocortical axon pathfinding, we developed a new mouse model to constitutively remove Jnk2 and Jnk3, while leaving Jnk1 function intact (Fig. 3.9 C). NetrinG1-positive thalamocortical axons in the D/x5/6-CIE;Jnk2-/;Jnk3-/- mouse extended through the internal capsule and reached the cortex at E15.5 (Fig. 3.9 C, $n=3 / 3$ ) and at P0 (Fig. 3.S5 C, $n=3 / 3$ ) in levels comparable to control brains (Fig. 9A, $n=4 / 4$, Fig. 3.S5 A, n=5/5). We next asked whether the removal of Jnk1 from the $D / x 5 / 6$ territory alone could be causing the thalamocortical axon misrouting. In the D/x5/6-CIE;Jnk1fl/fl mouse (Fig. 3.9D), where $J n k 1$ is conditionally removed from the $D / x 5 / 6$ territory but $J n k 2$ and $J n k 3$ remain intact, thalamocortical axons cross the DTB and enter the cortex (Fig. 3.9D, $n=3 / 3$ ). The removal of Jnk2 and Jnk3 constitutively, or the conditional removal of only one JNK gene, Jnk1, from the $D / x 5 / 6$ territory, are themselves insufficient to cause thalamocortical axon misrouting.

Since our data indicated that dosage of JNK gene function was an important component of the thalamocortical axon misrouting phenotype, we next asked whether a single copy of either Jnk1 or Jnk3 was sufficient for proper thalamocortical axon extension. In the D/x5/6CIE;Jnk1fl/+;Jnk2-/-;Jnk3-/- mouse (Fig. 3.9 E), only one copy of Jnk1 is conditionally removed from the $D / x 5 / 6$ lineage in a Jnk2/Jnk3 double knockout background. Thalamocortical axons are able to extend across the DTB and into the cortex in this mouse model at E15.5 (Fig. 3.9 E, $\mathrm{n}=5 / 5$ ) and P0 (Fig. 3.5 D, n=4/4), suggesting that only one copy of Jnk1 is needed in the D/x5/6 territory for proper thalamocortical axon extension. Similarly, in the DIx5/6-CIE;Jnk1fl/fl;Jnk2-/;Jnk3+/- mouse, where only one copy of Jnk3 is present, thalamocortical axons extend properly though the internal capsule and into the cortex (Fig. 3.9F, n=5/5). Retaining one copy of Jnk1 in the $D / x 5 / 6$ territory, or one copy of Jnk3 in the mouse, is sufficient for thalamocortical axons to cross the DTB and reach the cortex. Thus, it appears to be the complete loss of JNK function 
from the $D / x 5 / 6$ territory that leads to the non-autonomous misrouting of thalamocortical axons in cTKO mice. 


\section{Discussion}

In this current study, we show there is a non-autonomous requirement for JNK signaling in thalamocortical axon pathfinding. In the cTKO model, Jnk1 is conditionally removed from D/x5/6 lineage cells in a Jnk2/Jnk3 null background, which completely eliminates JNK signaling from the territory through which thalamocortical axons traverse. Beginning early in development, thalamocortical axons are unable to cross the diencephalon-telencephalon boundary (DTB) in cTKO brains, and instead misroute both ventrally into the hypothalamus as well as rostrally along the ventral surface of the telencephalon. Other longitudinal axon tracts in the $c T K O$ forebrain, including the corticothalamic, striatonigral, and nigrostriatal axons, are unable to cross the DTB, and fail to reach their intended targets through the appropriate pathway and in the correct number. Both the Islet1-postivie corridor cells and Nkx2.1-positive globus pallidus are mispositioned in the cTKO ventral telencephalon, and fail to extend caudally to the DTB region. Guidepost cells in the ventral telencephalon which normally project axons back to the dorsal thalamus are severely reduced in number in the cTKO brain. An allelic series of different genetic mouse models reinforced the non-autonomous requirement for JNK signaling in thalamocortical axon pathfinding, and demonstrated that all three JNK genes must be removed from the $D / x 5 / 6$ territory for thalamocortical axon misrouting to occur.

\section{Autonomous and non-autonomous requirements for JNK signaling in the development of major forebrain and midbrain axon trajectories}

The diencephalon-telencephalon boundary is a critical region in the developing forebrain through which thalamocortical, striatonigral, and nigrostriatal axons all have to traverse early in embryonic development. In the cTKO model, all three axon pathways are unable to extend past the DTB and reach their intended targets. However, the complete loss of JNK function in the cTKO model only occurs from the D/x5/6 lineage, which does not directly target the thalamus 
and thalamocortical axons, or the substantia nigra and nigrostriatal axons. The $D / x 5 / 6$ expression domain encompasses the lateral and medial ganglionic eminences in the ventral telencephalon, as well as portions of the prethalamus and hypothalamus (Panganiban and Rubenstein, 2002; Stenman et al., 2003). Therefore, striatonigral axons are directly impacted by the conditional deletion of JNK, and could themselves have an autonomous requirement for JNK function in axon extension and guidance. The loss of proper striatal connectivity could in turn cause thalamocortical and possibly even nigrostriatal axons to misroute. Indeed, in an OLprotocadherin knockout, the loss of striatal axons was shown to be responsible for thalamocortical axon misrouting (Uemura et al., 2007). Conditional removal of JNK function from the cells giving rise to striatal axons would need to be examined to determine if striatal axons have an autonomous requirement for JNK signaling, and if the striatal defects could be responsible for other axon misrouting phenotypes in the cTKO model.

Since the thalamus is not targeted by the conditional $D / \times 5 / 6$ deletion of Jnk1, and extension of thalamocortical axons is normal in the DIx5/6-CIE;Jnk2-/-;Jnk3-/- mouse model, this suggests there is a non-autonomous requirement for JNK signaling in the extension of thalamocortical axons. In the ventral telencephalon, the $D / x 5 / 6$ territory encompasses both corridor cells and guidepost neurons, two populations of cells known to non-autonomously influence thalamocortical axon pathfinding. The telencephalic corridor is formed by Islet1positive cells migrating ventromedially from the lateral ganglionic eminence, to form a permissive territory between the Nkx2.1-positive medial ganglionic eminence and globus pallidus (Lopez-Bendito et al., 2006). In the cTKO model, both Islet1- and Nkx2.1-positive cells fail to extend caudally towards the DTB in the telencephalon, and Islet1-positive cells aberrantly invade the globus pallidus. Disruptions to the corridor have been documented in other models with thalamocortical axon misrouting phenotypes (Bielle et al., 2011a; Lopez-Bendito et al., 2006; Morello et al., 2015; Simpson et al., 2009; Uemura et al., 2007), and may be a reason why thalamocortical axons don't extend across the DTB in the cTKO mouse. 
A second population of cells in the ventral telencephalon thought to be important in early thalamocortical axon guidance are guidepost neurons, which extend axons back to the dorsal thalamus (Braisted et al., 1999; Metin and Godement, 1996; Molnar and Cordery, 1999). In the cTKO model, these cells are severely reduced in number, similar to other models in which thalamocortical axons fail to extend across the DTB (Jones et al., 2002; Lakhina et al., 2007; Mitsogiannis et al., 2017; Tuttle et al., 1999; Uemura et al., 2007). Interestingly, some of these backfilled cells were found to be D/x5/6-positive in another model, which were then missing from the ventral telencephalon with the D/x5/6-mediated deletion of Celsr3 (Feng et al., 2016). These backfilled cells reside in the D/x5/6 territory in the cTKO model, and the severe reduction in number of these cells may impact early thalamocortical axon guidance. The loss of JNK signaling in the $D / x 5 / 6$ territory is directly impacting corridor and guidepost neurons, which is likely non-autonomously impacting thalamocortical axon pathfinding.

\section{Non-canonical WNT signaling in thalamocortical axon extension}

The Planar Cell Polarity (PCP) pathway is a component of non-canonical Wnt signaling, known for its roles in tissue morphogenesis, cilia organization, and directed cell migration (Simons and Mlodzik, 2008; Wang and Nathans, 2007). Two seven-transmembrane receptors for Wnt ligands in the PCP pathway, Frizzled3 (Goodrich and Strutt, 2011; Vinson et al., 1989) and Celsr3 (Usui et al., 1999), were both shown to play novel roles in axon guidance (Tissir et al., 2005; Wang et al., 2002), however the mechanism through which this occurs is currently unknown. Interestingly, after Frizzled3 recruits Dishevelled inside of the cell, one of the main signaling cascades that is part of the PCP pathway is the binding of this complex to Rac1, which activates JNK signaling (Niehrs, 2012).

In both the Frizzled3 and Celsr3 knockout mice, there are severe disruptions to major axon pathways including the thalamocortical, corticothalamic, and nigrostriatal tracts, and a near 
complete breakdown of the internal capsule (Fenstermaker et al., 2010; Tissir et al., 2005;

Wang et al., 2002; Wang et al., 2006). These are very reminiscent of the cTKO mouse, in which thalamocortical and corticothalamic axons fail to cross the internal capsule, and nigrostriatal axons are misrouted at the level of the hypothalamus. Interestingly, Frizzled3 and Celsr3 have been conditionally removed using the D/x5/6 driver line, and nearly identical phenotypes have been reported (Hua et al., 2014; Qu et al., 2014; Zhou et al., 2008; Zhou et al., 2009). However, when Celsr3 or Frizzed3 were deleted in both the cortex and the thalamus, longitudinal axon pathways developed normally, suggesting it is the expression of the PCP proteins in the territory through which axons traverse and not the axons themselves that is critical for their proper guidance (Qu et al., 2014).

Recent studies have looked more closely at the intermediate target territories in both of these mutants. Ventral telencephalic guidepost cells were missing or severely reduced in the Celsr3 (Jia et al., 2014) and DIx5/6;Celsr3fl/fl (Feng et al., 2016) mice, respectively, similar to the severe reduction observed in the cTKO forebrain. In the cTKO mouse, the telencephalic Islet-positive corridor cells invaded the globus pallidus, which was also observed in the Celsr3 (Jia et al., 2014) and Frizzled3 (Morello et al., 2015) mutants. Additionally, in both the Celsr3 and cTKO mutants, Nkx2.1-positive cells failed to extend caudally to the DTB region (Jia et al., 2014). Finally, striatal connections were compromised in both Celsr3-/- and Frizzled3-/- mice (Jia et al., 2014; Morello et al., 2015), which was reminiscent of the Darpp32-positive axons being unable to extend past the DTB in the cTKO mutant. Future studies will aim to determine if the axon phenotypes observed in mutants of the PCP genes act upstream of the JNK signaling pathway to cause the axon misrouting phenotypes observed in all three mutants. 


\section{Axon guidance cues and gradients in thalamocortical axon pathfinding}

Gradients of attractive and repulsive cues in the telencephalon, including netrins, slits, ephrins, and semaphorins, guide the sorting of thalamocortical axons once they reach the subpallium. Disruptions to these guidance cues have resulted in the mis-targeting of thalamocortical axons to different cortical areas. Since the conditional deletion of JNK signaling in the cTKO mouse is targeting the ventral telencephalon, there may be a disruption to the patterning of guidance cues, which could affect the sorting of thalamocortical axons that are able to enter the telencephalon at later embryonic and early postnatal time points in ectopic positions.

Netrins are diffusible guidance cues, expressed in an anterior-high posterior-low gradient in the ventral telencephalon (Braisted et al., 2000). DCC (deleted in colorectal cancer), Unc5, and FLRT3 (fibronectin leucine rich transmembrane) receptors, among others, on thalamocortical axons use the Netrin gradient once in the telencephalon to fan-out along the anterior-posterior axis to target the appropriate cortical areas (Leyva-Diaz et al., 2014; Powell et al., 2008). In Netrin-/- mice, thalamocortical axons are disorganized in the ventral telencephalon, and fewer thalamic axons reach the cortex (Braisted et al., 2000; Powell et al., 2008), similar to the cTKO mouse here. Interestingly, Netrin1 has been shown to increase JNK activity in neurons, and removal of JNK signaling inhibited axon outgrowth induced by Netrin1, suggesting JNK acts downstream of Netrin signaling in axon guidance (Qu et al., 2013).

Slit2 is another guidance molecule that is expressed in an anterior-high, posterior-low gradient, but is restricted to the corridor region in the ventral telencephalon. It can guide the positioning of corridor cells in the ventral telencephalon through expression in the ventral medial ganglionic eminence and preoptic area (Bielle et al., 2011a). In Slit2-/- mice, the corridor fails to extend caudally to the DTB, similar to the cTKO mouse, and as a result, thalamocortical axons fail to cross the DTB and instead misroute into the hypothalamus (Bielle et al., 2011a). JNK 
acts upstream of Slit/Robo signaling in Drosophila (lida et al., 2019; Vaughen and Igaki, 2016), however the connection between the two pathways in mouse embryogenesis remains unknown.

In addition to their expression in the telencephalon, both Netrins (Kennedy et al., 1994; Kennedy et al., 2006) and Slits (Bagri et al., 2002) are also expressed along the midline in the hypothalamus. The presence of these guidance molecules normally prevents axons from inappropriately crossing the midline. However, in Dcc-/- (Xu et al., 2010a) and Slit1-/-;Slit2-/mice (Bagri et al., 2002), TH-positive nigrostriatal axons mis-project ventrally into a "U-shaped" bundle in the hypothalamus, instead of coursing rostrally into the telencephalon. The THpositive axons in the Dcc-/- and Slit1-/-;Slit2-/- mice aberrantly course through the hypothalamus at a similar location to the "U-shaped" bundle of nigrostriatal axons observed in the cTKO model. The D/x5/6-targeted deletion of JNK signaling also encompasses the hypothalamus, suggesting chemo-repulsive cues normally present at the midline may be compromised in cTKO mice.

In addition to their known roles in axon guidance, recent work has shown that crosstalk between different guidance molecules can regulate axonal pathfinding (Stoeckli, 2018). For instance, thalamocortical axons respond differently to slits and netrins when the two gradients are presented in combination with one another (Bielle et al., 2011b; Dupin et al., 2015; LeyvaDiaz et al., 2014), suggesting that common mechanisms may underly axons' responsiveness to multiple guidance cues. Trio, a guanine nucleotide exchange factor that activates Rac1 and RhoA downstream of Netrin (DeGeer et al., 2015) and Slit2 (Backer et al., 2018) signaling, respectively, was shown to be critical for the correct formation of the corridor and the thalamocortical axon pathway in a mouse model (Backer et al., 2018). Interestingly, JNK is a downstream target of both Rac1 (Coso et al., 1995; Minden et al., 1995; Rosso et al., 2005) and RhoA (Marinissen et al., 2004; Tang et al., 2018). Future studies are needed to unravel whether JNK signaling could be playing a role in one of the classical axon guidance signaling 
pathways, or whether it could even act as a common downstream target, serving as a link between different guidance molecules.

\section{Conclusions}

Thalamocortical axons extend a long distance through many different territories to reach their cortical targets, and disruptions at any point along this path can lead to lasting changes in cortical circuitry. Here, we identified a novel, non-autonomous requirement for JNK signaling in the early pathfinding of thalamocortical axons. Elucidation of upstream and downstream regulators in this critical developmental process will further enhance our understanding of the establishment of thalamocortical connectivity, and in turn how neurological diseases may arise. 


\section{Materials and Methods}

Animals

The Office of Laboratory Animal Resources at West Virginia University housed and cared for mice (Mus musculus) used in all experiments. All mouse procedures were approved by and performed in accordance with the Institutional Animal Care and Use Committee at West Virginia University. Mice in all crosses were acquired and maintained on a C57BL/6J background. The individual mouse strains are as follows: C57BL/6J (Stock \# 000664, The Jackson Laboratory); DIx5/6-Cre-IRES-EGFP (DIx5/6-CIE; (Stenman et al., 2003)), floxed Mapk8tm1Rjd mice (Jnk1fl/fl; (Das et al., 2007); kindly provided by Dr. Roger Davis); Mapk9tm1Flv (Jnk2-/-; Stock \# 004321, The Jackson Laboratory); Mapk10tm1Flv (Jnk3-/-; Stock \# 004322, The Jackson Laboratory). DIx5/6-CIE control animals were generated by crossing C57BL/6J dams with DIx5/6-CIE hemizygous males. Jnk1fl/fl; Jnk2-/-; Jnk3-/- dams were mated with DIx5/6-CIE; Jnk1fI/+; Jnk2-/-; Jnk3+/- males to generate conditional triple knockout (cTKO: DIx5/6-CIE; Jnk1fI/fl; Jnk2-/-; Jnk3-/-), DIx5/6-CIE; Jnk1fl/+; Jnk2-/-; Jnk3-/-, and DIx5/6-CIE; Jnk1fl/fl; Jnk2-/-; Jnk3+/- mice. Jnk2+/-; Jnk3-/- dams were mated with DIx5/6CIE; Jnk2-/-; Jnk3+/- males to generate DIX5/6-CIE; Jnk2-/-; Jnk3-/- animals. Jnk1f/fI dams were mated with DIx5/6-CIE; Jnk1fl/+ males to generate DIx5/6-CIE; Jnk1fl/fl animals.

\section{Tissue collection and processing}

Dams were placed into male cages each night, and checked every morning for a vaginal plug. The day of the vaginal plug was recorded as embryonic day 0.5 . For embryonic collections, timed-pregnant dams were euthanized by rapid cervical dislocation. Embryos were removed and placed in phosphate-buffered saline (PBS; $136.9 \mathrm{mmol} \mathrm{NaCl}, 2.683 \mathrm{mmol} \mathrm{KCl}$, 4.290mmol $\mathrm{Na}_{2} \mathrm{HPO}_{4} 7 \mathrm{H}_{2} \mathrm{O}, 1.470 \mathrm{mmol} \mathrm{KH}_{2} \mathrm{PO}_{4}$ ), and tissue for genotyping was taken. $\mathrm{E} 12.5$ and E13.5 whole heads were collected into 4\% paraformaldehyde (PFA; Millipore Sigma, 818715). E15.5 and E17.5 brains were dissected out from the heads, then placed into 4\% PFA. 
For P0 collections, newborn pups were deeply anesthetized on ice, and transcardially perfused with 1 X PBS followed by $4 \%$ PFA. Brains were dissected out from the head, and placed in $4 \%$ PFA on ice. All collected material was fixed for 24 hours in $4 \%$ PFA at $4{ }^{\circ} \mathrm{C}$, before being transferred through a sucrose series $(10 \%, 20 \%, 30 \%)$ for cryoprotection. Material was then embedded in Tissue Freezing Medium (VWR 15146-019), frozen, and stored at $-80^{\circ} \mathrm{C}$. Frozen material was sectioned at $12 \mu \mathrm{m}$ on a Leica cryostat (CM3050S) onto superfrost plus slides (Fisher, 12-550-15), and stored at $-20^{\circ} \mathrm{C}$ prior to staining.

\section{Immunohistochemistry and Imaging}

Slides were outlined with a pap pen (Electron Microscopy Sciences, 71310), and rehydrated for 20 mins with 1 X PBS, followed by permealization buffer with either $5 \%$ normal donkey (EMD Millipore, S30-100ml) or goat serum (Thermofisher scientific, 16210064). Primary antibody was diluted in block, placed on the slides, and left to incubate overnight at $4^{\circ} \mathrm{C}$. Slides were then rinsed with $1 \mathrm{X}$ PBS, followed by incubation with secondary antibodies diluted in block for 2 hours at room temperature. After incubation, slides were rinsed again with 1 X PBS, then stained with the nuclear counterstain Hoechst (Thermo Scientific $62249,1 \mu \mathrm{g} / \mathrm{ml}$ ) for 10 minutes. Slides were rinsed before being cover-slipped with mounting media (Mowiol 4-88, Polysciences, 17951) containing an anti-fade reagent (p-Phenylenediamine, Fisher Scientific, AC130570050). All slides were stored at $4^{\circ} \mathrm{C}$ prior to imaging. Brain sections were imaged using an Olympus VS120 Slide Scanner with a UPLSAPO 10x objective, as well as on a Zeiss 710 confocal microscope with a 20x Plan-Apo objective lens.

\section{Dil}

E13.5. E13.5 heads were collected as above, and placed in $4 \%$ PFA overnight at $4^{\circ} \mathrm{C}$. Following fixation, head were rinsed into $1 \mathrm{X}$ PBS. Whole heads were hemi-sected along the mid-sagittal plane, and a Dil crystal (Invitrogen, D3911) was placed into the dorsal thalamus of both halves of the brain. The heads incubated at $37^{\circ} \mathrm{C}$ for 2 weeks in the dark in $1 \mathrm{X}$ PBS. 
Following incubation, each half was embedded in 3\% agarose (Fisher Scientific, BP165-25), sliced at $150 \mu \mathrm{m}$ on a Leica vibratome (VT1000S), serially collected, and mounted onto slides. E15.5. E15.5 brains were collected as above, and placed in $1.5 \%$ PFA overnight at $4^{\circ} \mathrm{C}$. After being rinsed into $1 \mathrm{X}$ PBS, brains were embedded in $3 \%$ agarose, then sectioned coronally at $300 \mu \mathrm{m}$ on a vibratome. Dil crystals were placed into one half of the dorsal thalamus in the slices. Brain slices were allowed to fill for 3 days at room temperature in 1 X PBS in the dark before being mounted onto slides.

P0. P0 mice were perfused as described above, and brains were placed in 4\% PFA for 1 week at $4^{\circ} \mathrm{C}$. Brains were then rinsed into $1 X$ PBS and hemi-sected along the mid-sagittal plane. Dil crystals were placed into the cortex of one half of the brain, and into the dorsal thalamus in the other half. Brains incubated at $37^{\circ} \mathrm{C}$ for 8 weeks in the dark. Each half of the brain was embedded in $3 \%$ agarose, sectioned at $200 \mu \mathrm{m}$ on a vibratome, then slide mounted before imaging.

All images from Dil placements were acquired using a Zeiss 710 confocal microscope with a 20x Plan-Apo objective lens.

\section{CT scans}

P0 mice were perfused as above. Whole heads were placed in 4\% PFA for 2 days at $4^{\circ} \mathrm{C}$, after which they were transferred to stability buffer ( $4 \% \mathrm{w} / \mathrm{v}$ paraformaldehyde $(\mathrm{pH} 7.2), 4 \%$ w/v acrylamide (Bio-Rad 1610140), 0.05\% w/v bis-acrylamide (Bio-Rad 1610142), 0.25\% w/v VA044 initiator (Fisher Scientific, NC0632395), 0.05\% w/v Saponin (EMD Millipore, 558255), in 1x PBS) for 3 days at $4^{\circ} \mathrm{C}$ (Hsu et al., 2016). The conical containing the heads in stability buffer underwent nitrogen desiccation (3 minutes vacuum, 3 minutes Nitrogen, 3 minutes vacuum), and was then placed in a water bath at $37^{\circ} \mathrm{C}$ for 3 hours. Heads were carefully removed from the stability buffer, and placed in $0.1 \mathrm{~N}$ iodine (Thermo Fisher Scientific, AC12422) to stain for 2 days, with iodine being replaced at 24 hours. The heads were embedded in $3 \%$ agarose, then 
were imaged on a Bruker SkyScan 1272 MicroCT scanner (Al 0.5 mm filter, 850 ms exposure, and $7 \mu \mathrm{m}$ resolution).

Primary Antibodies:

\begin{tabular}{|l|l|l|l|l|}
\hline Host: & Antigen: & Concentration: & Company: & $\begin{array}{l}\text { Product } \\
\text { Number: }\end{array}$ \\
\hline Rabbit & Calbindin & $1: 2000$ & Swant & CB38 \\
\hline Rabbit & Darpp-32 & $1: 1000$ & $\begin{array}{l}\text { Syanptic } \\
\text { Systems }\end{array}$ & 382002 \\
\hline Chicken & GFP & $1: 1500$ & Abcam & ab13970 \\
\hline Goat & Islet1 & $1: 500$ & R\&D Systems & AF1837 \\
\hline Rabbit & L1 & $1: 250$ & $\begin{array}{l}\text { Aviva Systems } \\
\text { Biology }\end{array}$ & ARP63103 \\
\hline Goat & NetrinG1 & $1: 250$ & R\&D Systems & AF1166 \\
\hline Rabbit & Nkx2.1 & $1: 500$ & Santa Cruz & SC-13040 \\
\hline Rabbit & p-JNK & $1: 1000$ & Promega & V7931 \\
\hline Mouse & Tag1 & $1: 12.5$ & DSHB & AB_2315433 \\
\hline Chicken & TH & $1: 1000$ & Aves Labs & TYH \\
\hline
\end{tabular}

Secondary Antibodies:

\begin{tabular}{|l|l|l|l|}
\hline Antibody: & Concentration: & Company: & Product Number: \\
\hline Donkey anti-Chicken 488 & $1: 4000$ & $\begin{array}{l}\text { Jackson Immuno } \\
\text { Research }\end{array}$ & 703-545-155 \\
\hline Goat anti-Chicken 488 & $1: 4000$ & Invitrogen & A11039 \\
\hline Donkey anti-Goat 546 & $1: 2000$ & Invitrogen & A11056 \\
\hline Goat anti-Chicken 546 & $1: 2000$ & Invitrogen & A11040 \\
\hline Goat anti-Mouse 546 & $1: 2000$ & Invitrogen & A11003 \\
\hline Goat anti-Rabbit 546 & $1: 2000$ & Invitrogen & A11010 \\
\hline Donkey anti-Chicken 647 & $1: 2000$ & $\begin{array}{l}\text { Jackson Immuno } \\
\text { Research }\end{array}$ & $703-605-155$ \\
\hline Donkey anti-Rabbit 647 & $1: 2000$ & $\begin{array}{l}\text { Jackson Immuno } \\
\text { Research }\end{array}$ & $711-605-152$ \\
\hline Goat anti-Rabbit 647 & $1: 2000$ & Invitrogen & A21244 \\
\hline
\end{tabular}

\section{Acknowledgements}

We would like to thank Amanda Ammer and Karen Martin for their excellent assistance with microscopy. Imaging was performed in the WVU Imaging Facilities, which have been supported by the WVU Cancer Institute, the WVU HSC Office of Research and Graduate Education, and 
NIH grants P20RR016440, P30GM103488, U54GM104942, P20GM103434, and P30GM103503.

\section{Competing Interests}

The authors declare that no competing interests exist.

\section{Author Contributions}

Jessica Cunningham, Conceptualization, Methodology, Formal analysis, Investigation, Writingoriginal draft preparation, Visualization, Supervision; James Scripter, Formal analysis, Investigation; Stephany Nti, Investigation; Eric Tucker, Conceptualization, Methodology, Supervision, Funding acquisition.

\section{Funding}

This work was funded by the National Institutes of Health grant R01NS082262 to Eric Tucker. 


\section{Figures}
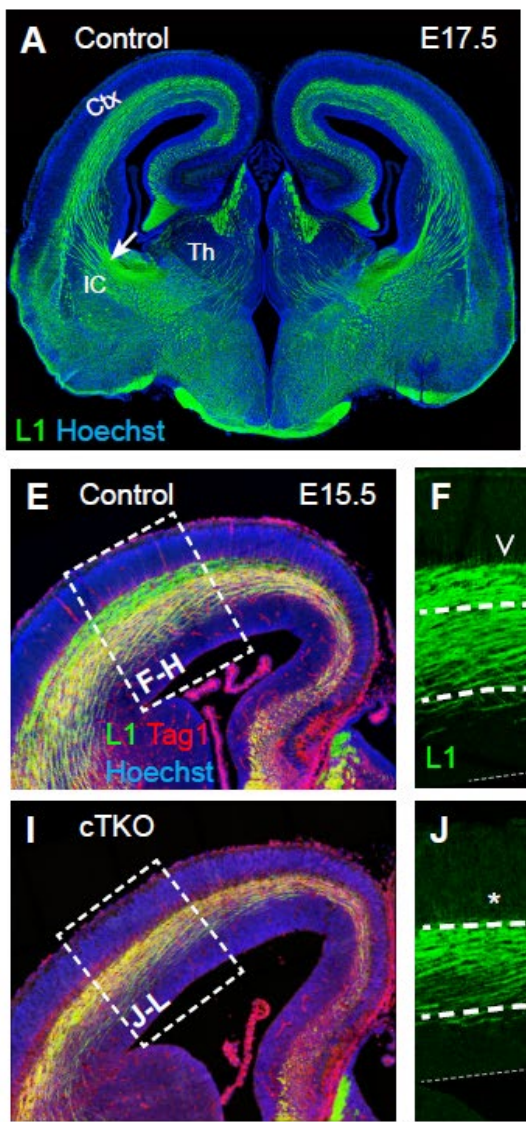
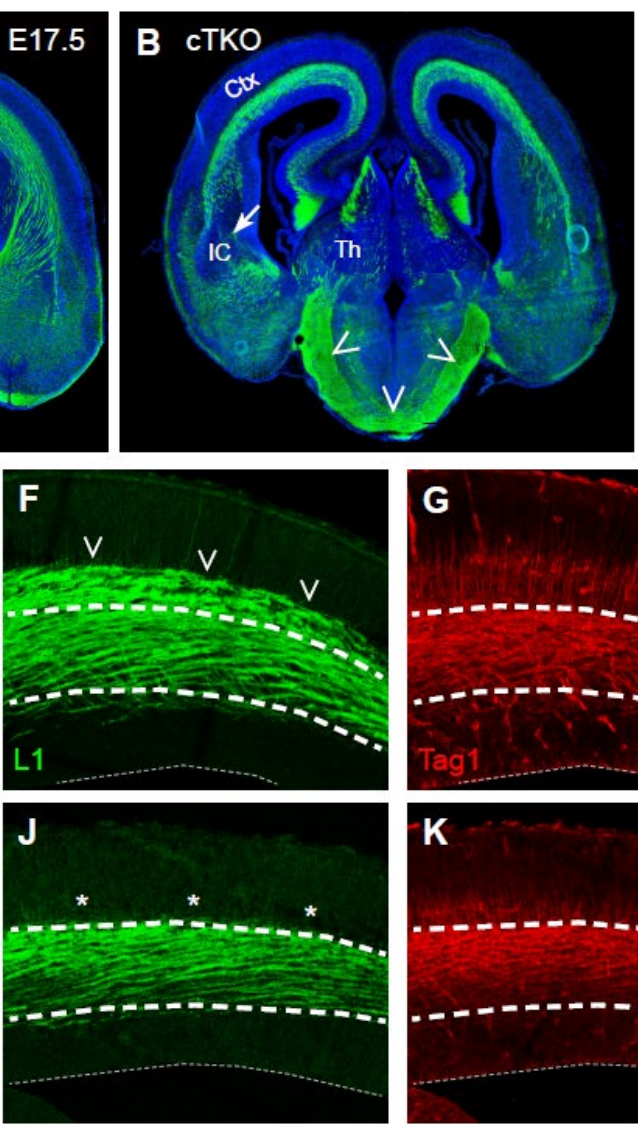
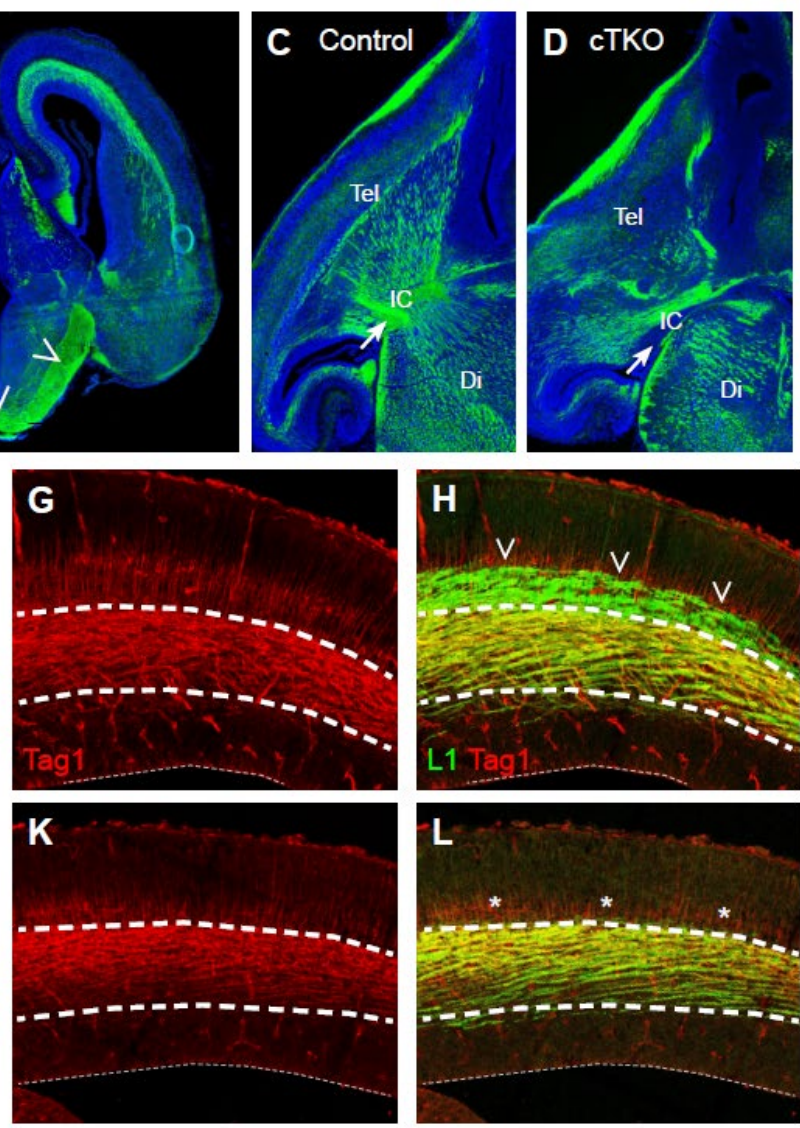

Figure 3.1: Axons are missing or misrouted in the cTKO brain. A-D. Immunohistochemistry for L1 in E17.5 brain sections in coronal (A-B) and axial (C-D) planes. The internal capsule (arrow) is present in control $(\mathrm{A}, \mathrm{C})$ but not $c T K O(\mathrm{~B}, \mathrm{D})$ brains. An ectopic "U-shaped" bundle projects into the hypothalamus in cTKO brains (open arrowheads, B). E-L. E15.5 cortices labeled with L1 and Tag1. L1-positve and Tag1-positive axons are present in control (E-H) and cTKO (I-L) cortices. A population of L1-positive/Tag1-negative axons is present in control brains (open arrowheads, F,H), but missing in cTKO brains (asterisks, J,L). Ctx- cortex, ICinternal capsule, Th- thalamus, Tel- telencephalon, Di- diencephalon. 

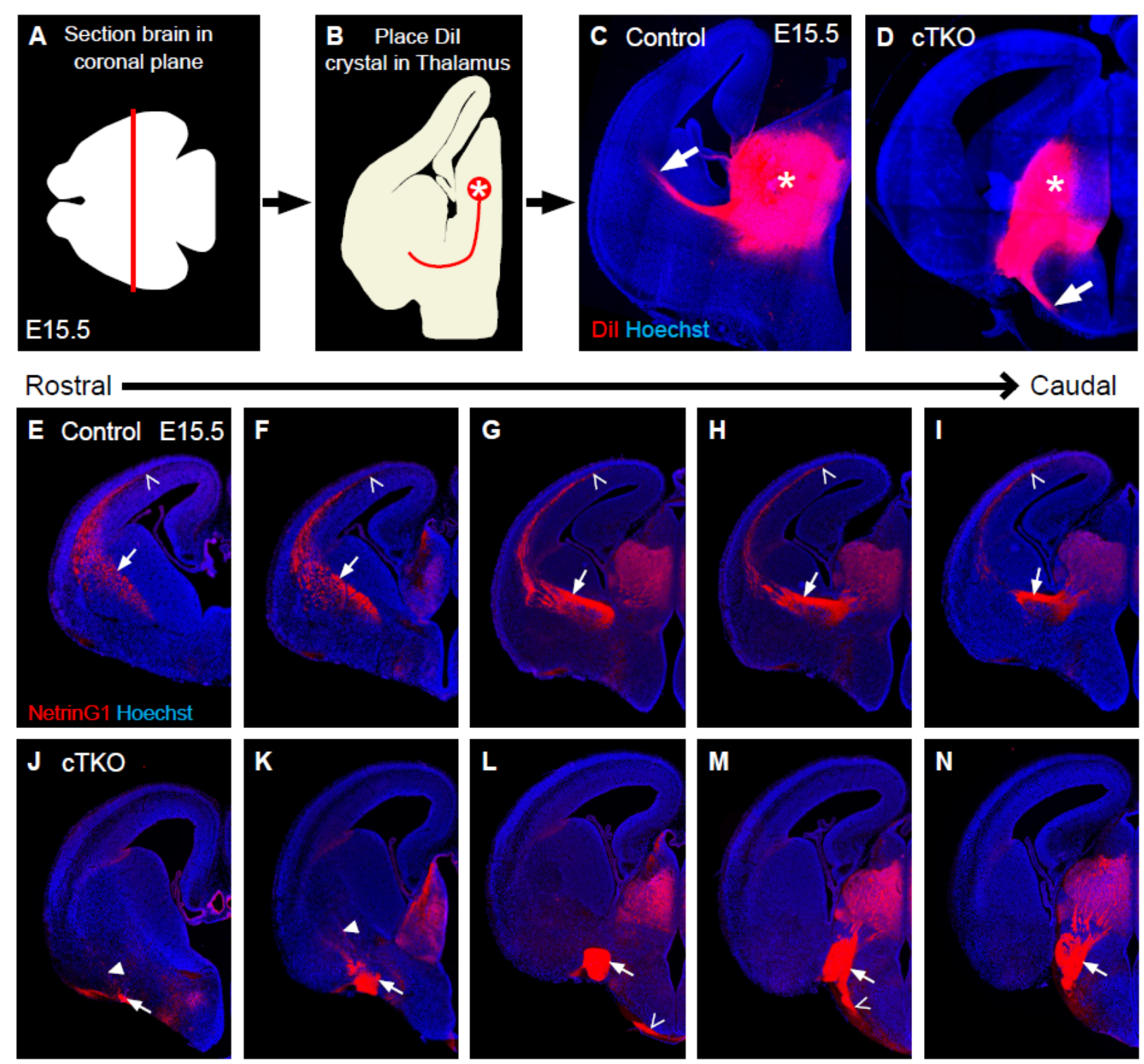

Figure 3.2: Thalamocortical axons are misrouted in cTKO brains. A-B. E15.5 brains are fixed and sectioned coronally at 300 microns, then a Dil crystal (asterisk) is placed into the dorsal thalamus. C-D. Dil labels axons projecting through the internal capsule in control brains (arrow, C), and axons projecting ventrally into the hypothalamus in cTKO brains (arrow, D). EN. NetrinG1-labeled thalamocortical axons in rostrocaudal series of control (E-I) and cTKO (J-N) brains at E15.5. In control brains, axons project through the internal capsule (arrow, E-I) and reach the cortex (open arrowheads, E-I). In cTKO brains, fibers project into the hypothalamus 
(open arrowheads, L-M), rostrally along the ventral telencephalon (arrows, J-N), and ectopically into the striatum (closed arrowheads, J-K). 

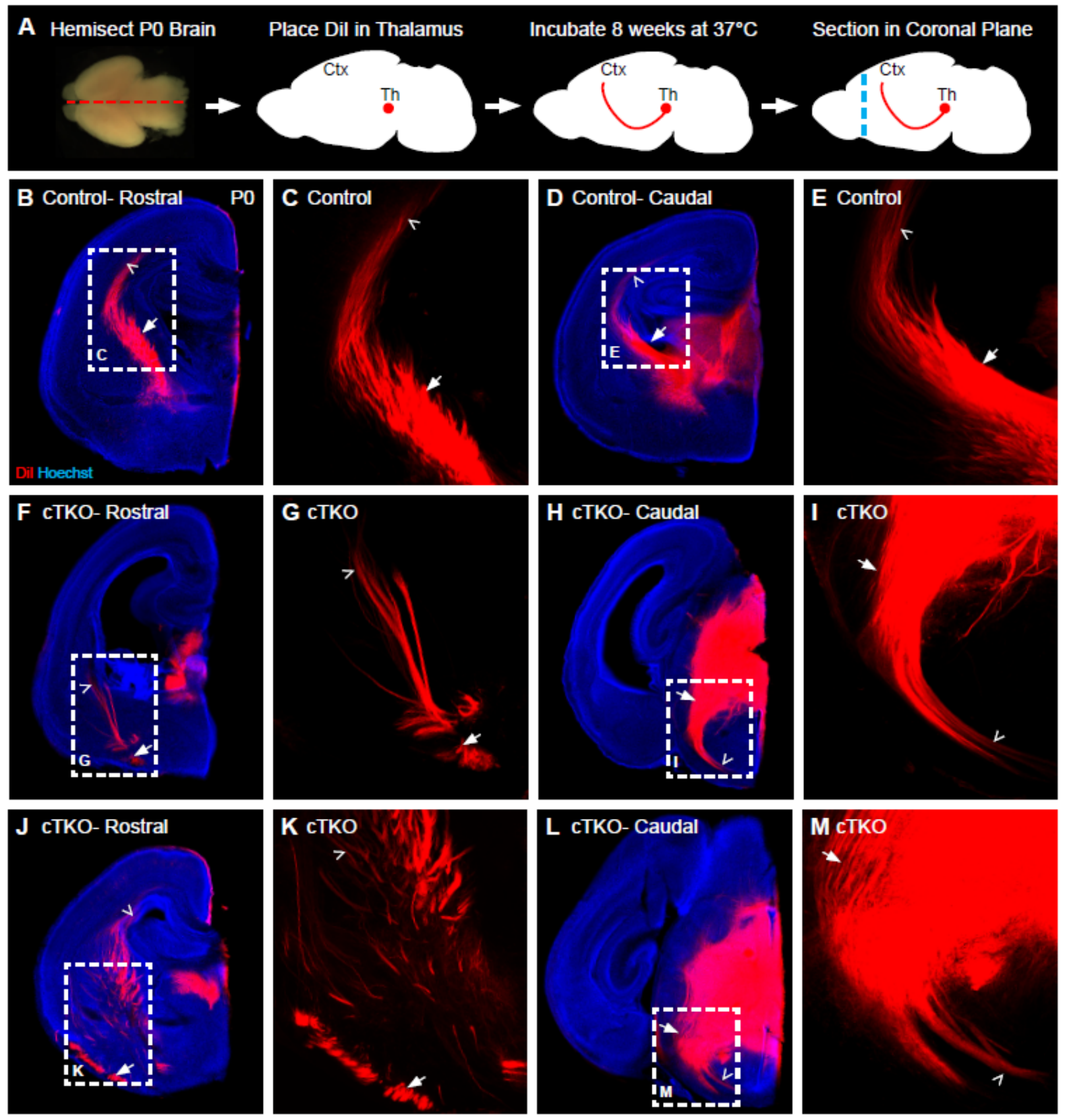

Figure 3.3: Axons projecting from the thalamus take aberrant trajectories in the PO cTKO

brain. A. PO brains were hemisected along the midsagittal plane, a Dil crystal was placed into the thalamus, and after incubation, brains were sectioned in the coronal plane at 200 microns. B-E. Dil-labeled axons in control brains project through the internal capsule (arrow) and reach the cortex (open arrowhead). F-M. Dil-labeled axons in cTKO brains project ventrally at caudal locations (arrow, H-I, L-M), and extend into the hypothalamus (open arrowhead, H-I, L-M). At 
more rostral levels ( $F-G, J-K)$, a bundle of axons resides along the ventral telencephalon (arrows). Some Dil-labeled axons in cTKO brains stop in the striatum (open arrowheads, F-G), and others are able to reach the cortex (open arrowheads, J-K). 


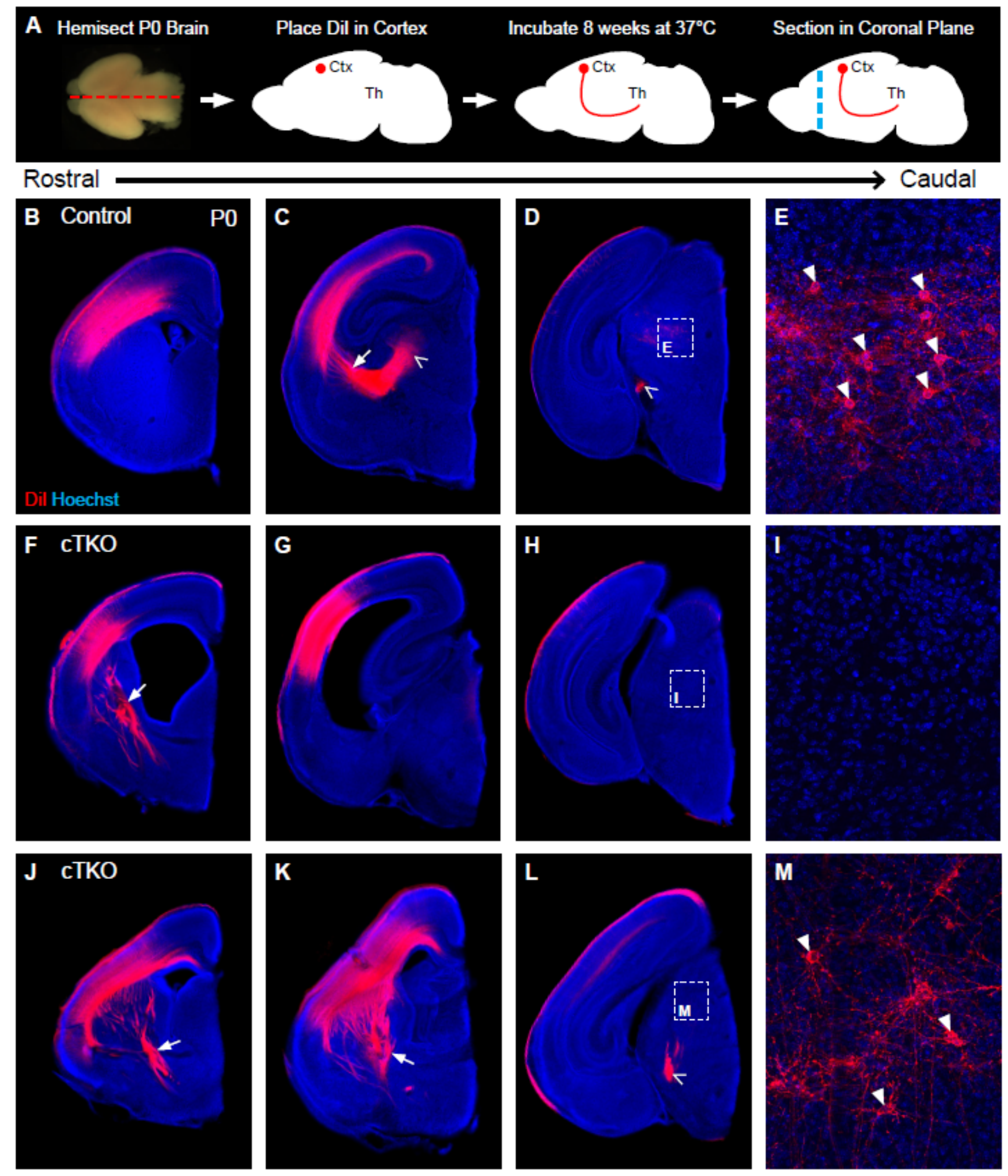

Figure 3.4: JNK signaling is required for proper corticothalamic axon extension. A. P0 brains were hemisected along the midsagittal plane, a Dil crystal was placed into the cortex, and after incubation, brains were sectioned in the coronal plane at 200 microns. B-E. Dil- 
labeled cortical axons in control brains project through the internal capsule (arrow, C) and into the thalamus (open arrowheads, C-D). Back-filled cells are present in the thalamus (closed arrowheads, E). F-M. Dil-labeled cortical axons in cTKO brains project rostrally and ventrally in a large fascicle (arrows, $\mathrm{F}, \mathrm{J}-\mathrm{K}$ ). In $\mathrm{n}=2 / 4$ cTKO brains (F-I), no cortical axons reach the thalamus $(\mathrm{G}-\mathrm{H})$, and no backfilled cells are present (I). In $\mathrm{n}=2 / 4$ cTKO brains (J-M), axons reach the thalamus (L), and backfilled cells are present (closed arrowheads, M). 

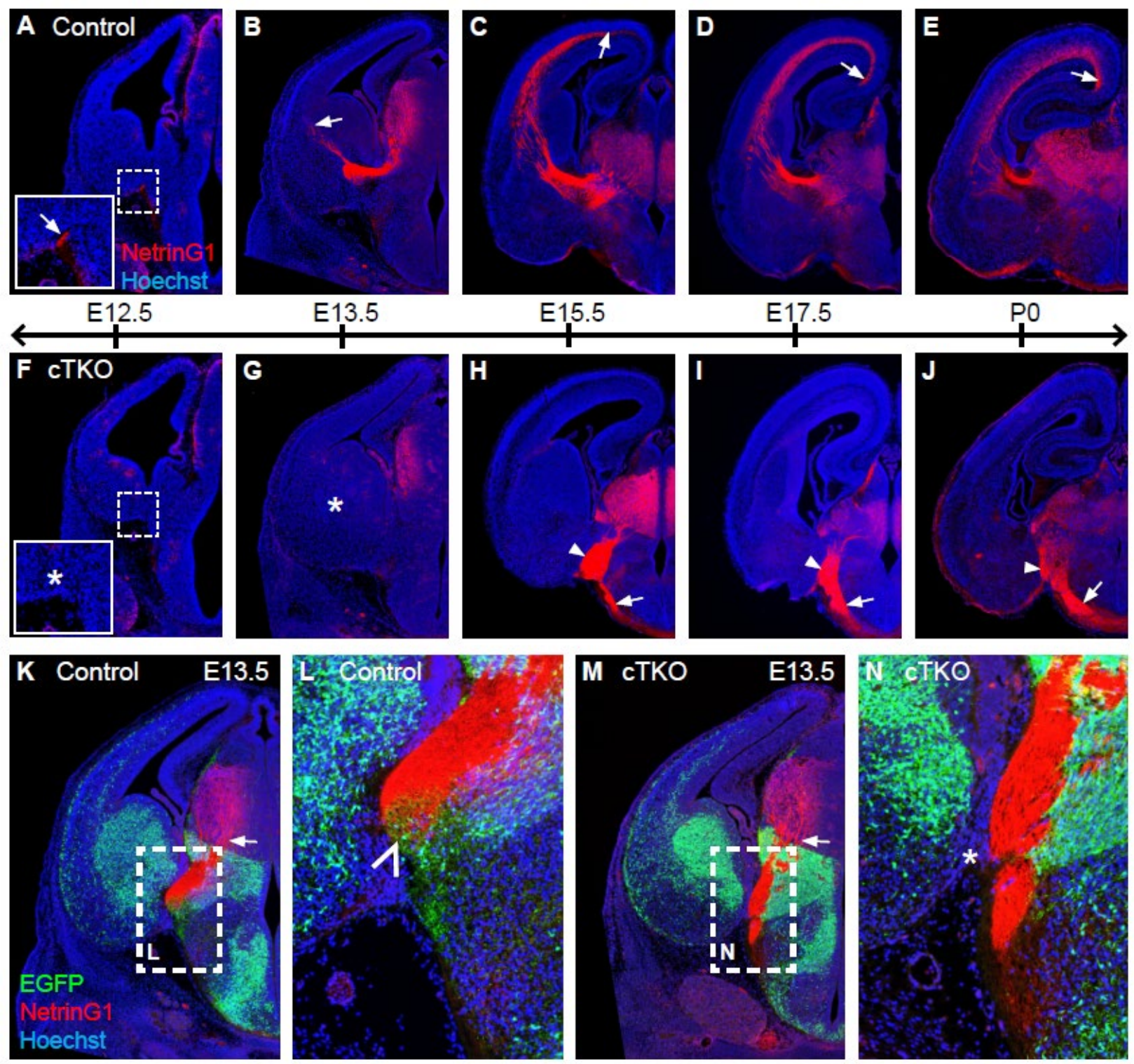

Figure 3.5: Thalamocortical axons are misrouted throughout early development in the

cTKO brain. A-E. NetrinG1 labeling of thalamocortical axons from E12.5 to P0 in control brains. Axons extend into the telencephalon at E12.5 (arrow-inset, A), reach the pallialsubpallial boundary at E13.5 (arrow, B), and continue to extend into the cortex from E15.5-P0 (arrows, C-E). F-G. In cTKO brains, NetrinG1-positive axons are missing in the telencephalon at E12.5 (asterisk, F) and E13.5 (asterisk, G). H-J. Axons are present at the DTB (closed arrowheads) or below the hypothalamus (arrows) in cTKO mice. K-L. NetrinG1-positive thalamocortical axons project from the thalamus (arrow, K), and cross into the telencephalon at 
the DTB on top of D/x5/6-positive cells and axons (open arrowhead, L) at E13.5. M-N. In cTKO brains, thalamocortical axons have extended from the thalamus (arrow, M), however have not crossed the DTB, which is missing D/x5/6-CIE cells and axons (open arrowhead, $\mathrm{N}$ ). 

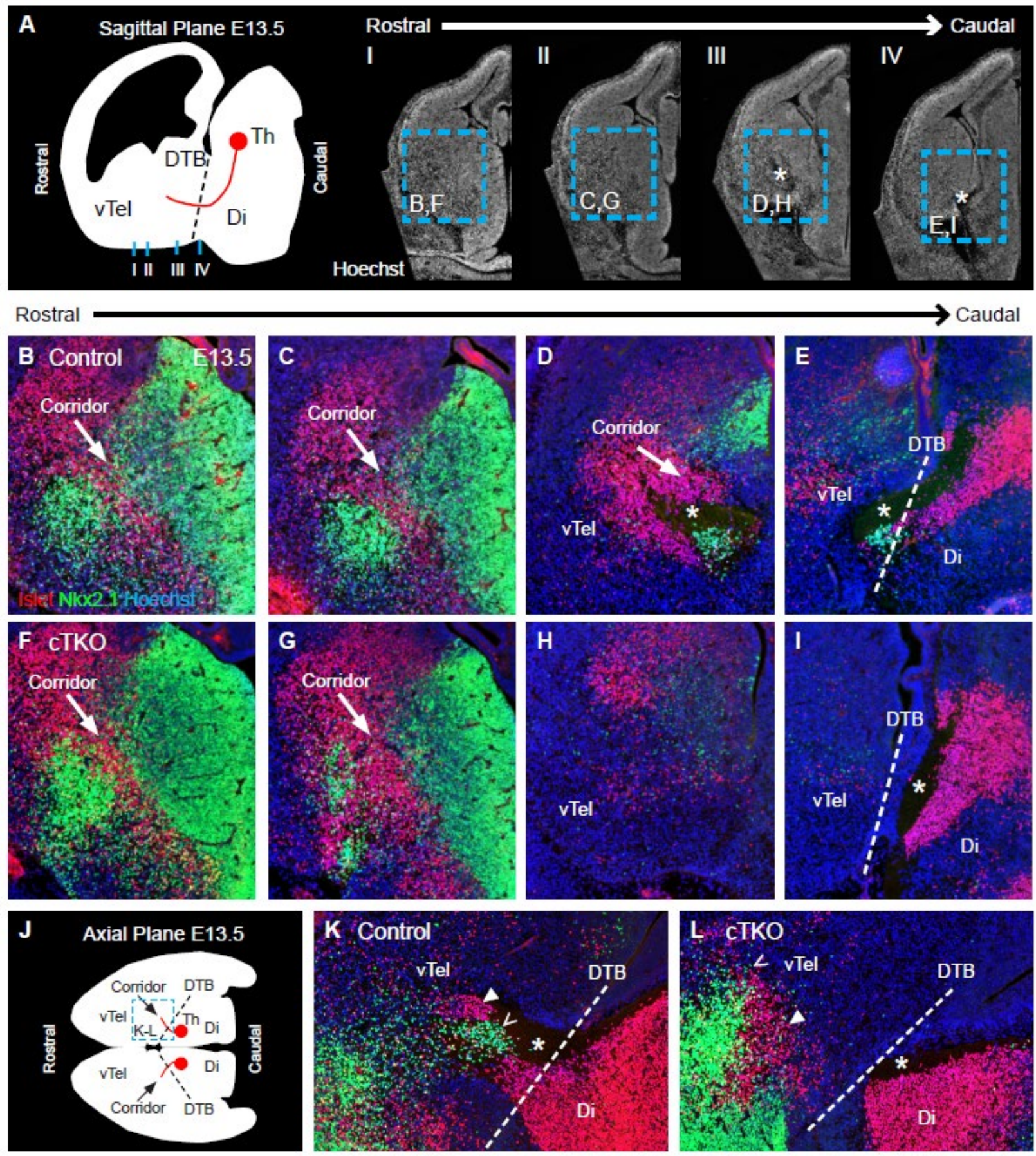

Figure 3.6: The corridor is malformed along the rostrocaudal axis in cTKO brains at

E13.5. A. Schematic diagram and Hoechst-labeled slices of the four positions analyzed for Islet1 and Nkx2.1 at E13.5. B-D. Islet1-positive cells form a permissive corridor between two Nkx2.1-positive regions (medial ganglionic eminence and globus palidus) through which 
thalamocortical axons extend (asterisk, D). E. Thalamocortical axons (asterisk) cross the diencephalon-telencephalon boundary (DTB) on top of Nkx2.1-positive cells, and Islet-positive cells extending from the diencephalon. F-I. In cTKO brains, the Islet1-positive corridor is present at rostral levels (arrows, F-G), but is greatly reduced at caudal levels $(\mathrm{H}-\mathrm{I})$. The Nkx2.1positive domain is expanded at rostral levels (F) and diminishes at more caudal locations (G-I) in cTKO brains. I. Thalamocortical axons (asterisk) fail to cross the DTB. Islet1-positive cells are present in the diencephalon, but are missing at the DTB. J-L. Axial view of the corridor region at E13.5. Islet1- (closed arrowhead) and Nkx2.1- (open arrowhead) positive cells extend caudally to where thalamocortical axons (asterisk) cross the DTB in control brains (K), but stop at rostral levels in cTKO brains (L). vTel- ventral telencephalon, Di- diencephalon, DTBdiencephalon-telencephalon boundary. 

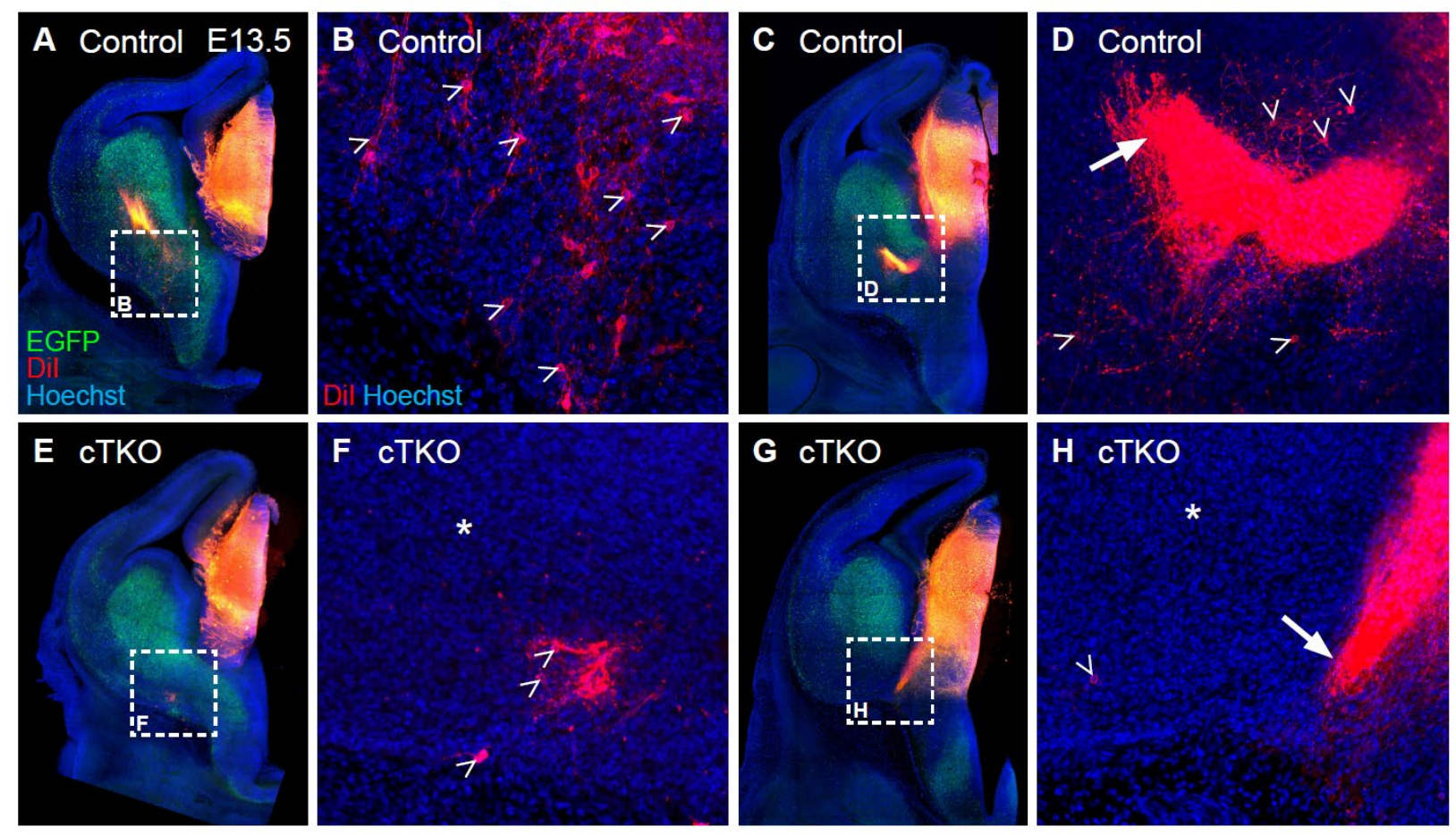

Figure 3.7: Ventral telencephalic guidepost cells that project to the dorsal thalamus are reduced in number in cTKO brains at E13.5. A-D. Dil-labeled thalamocortical axons and backfilled cells (open arrowheads, B,D) are present in the telencephalon after Dil placement in the dorsal thalamus at E13.5 in control brains. E-H. In cTKO brains, thalamocortical axons are missing in the telencephalon (asterisks, F,H), and instead project ventrally (arrow, $\mathrm{H}$ ). A few backfilled cells are present in the ventral telencephalon, but are reduced in number (open arrowheads, $F, H)$. 

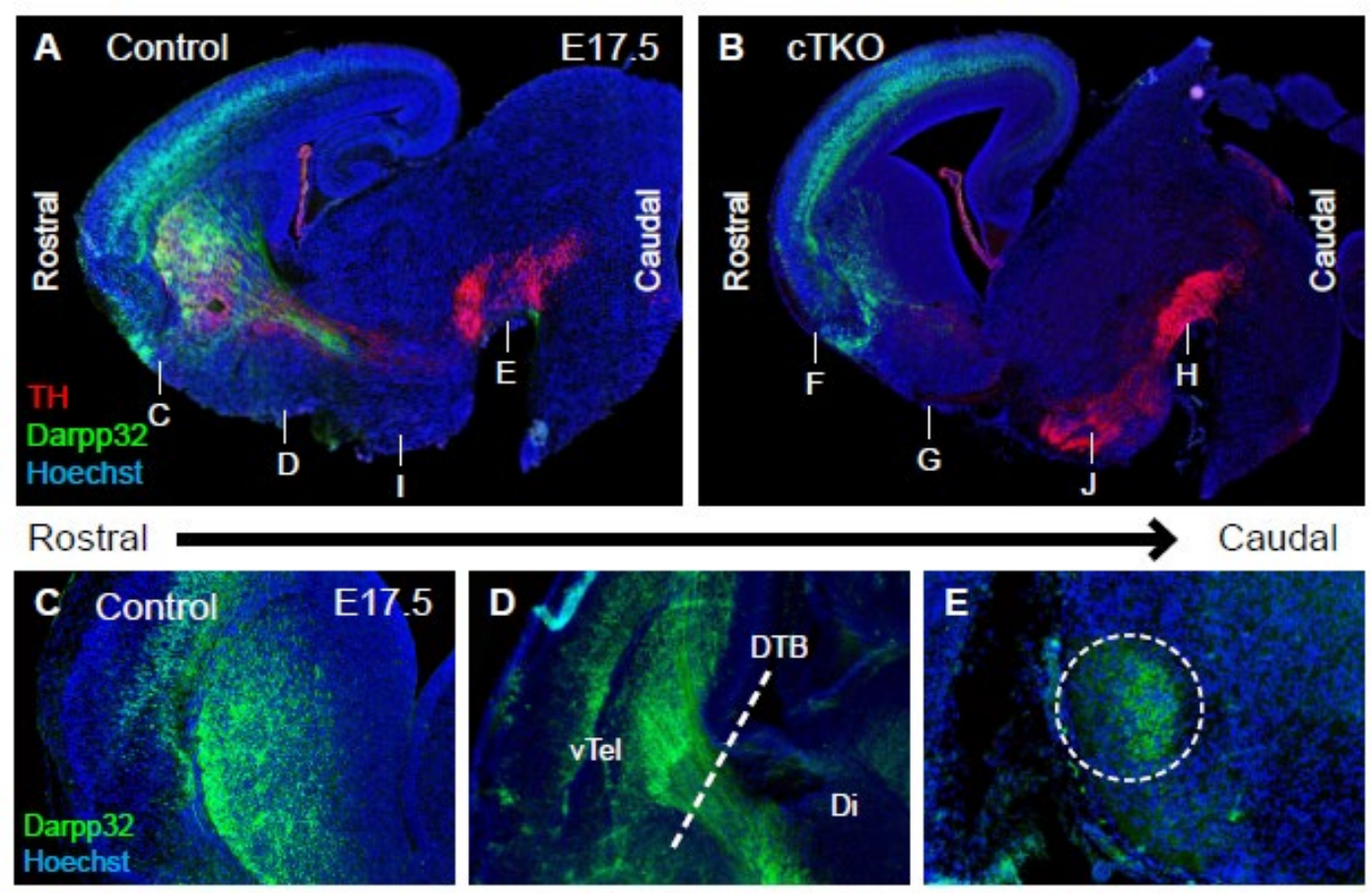

Caudal
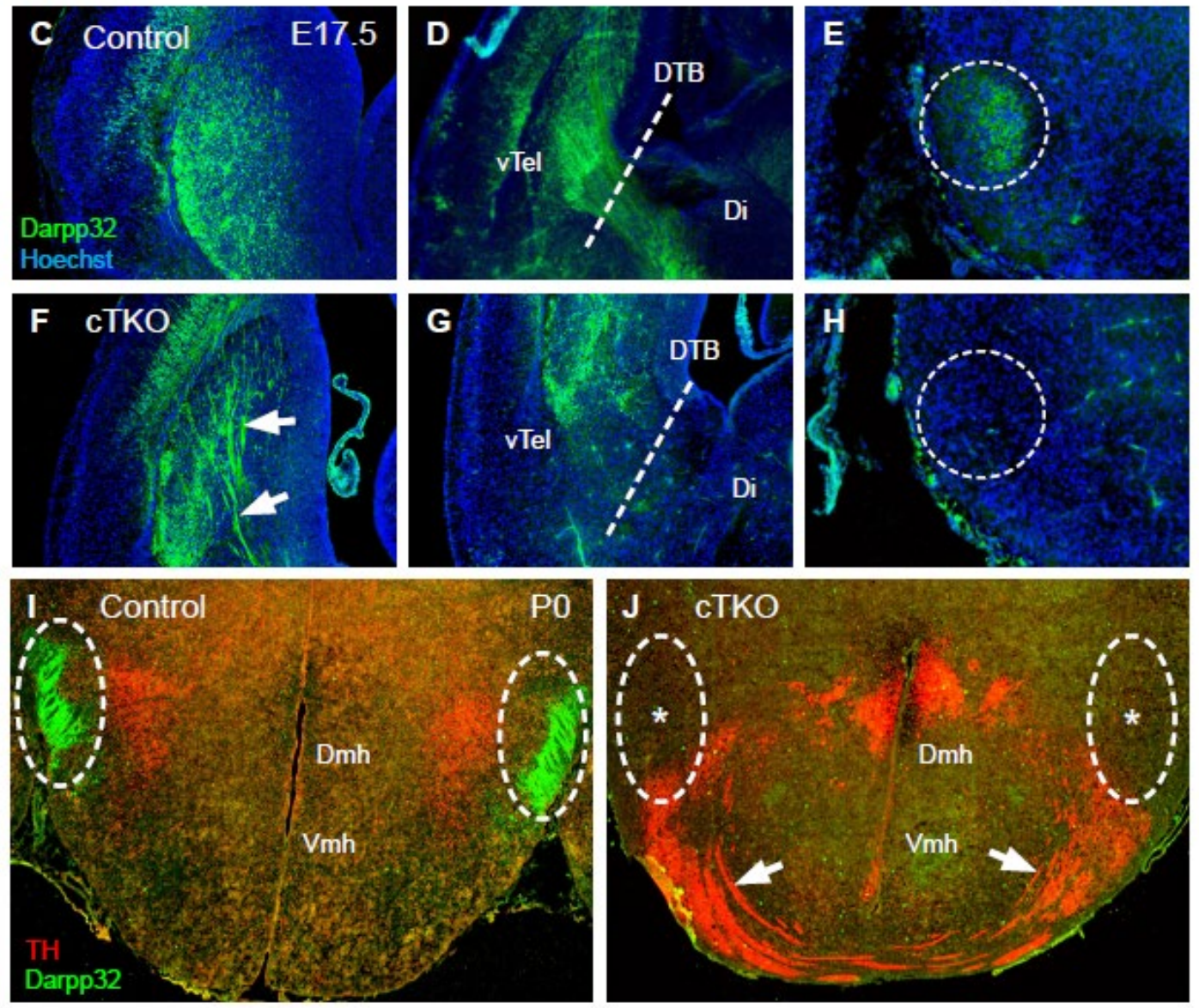

Figure 3.8: Axons fail to connect the striatum and the substantia nigra in cTKO brains. A.

Darpp32-positive striatonigral axons and TH-positive nigrostriatal axons project across the DTB in control brains at E17.5. B. Darpp32-postive axons are present in the telencephalon in cTKO brains but fail to cross the DTB, and TH-positive axons project ventrally. C-E. In control brains, Darrp32-positve axons are present in the striatum (C), cross the DTB (D), and reach the 
midbrain (E). F-H. In cTKO brains, striatal axons are misrouted in the striatum (arrows, F), do not cross the DTB $(\mathrm{G})$, and are absent in the midbrain $(\mathrm{H})$. I. At the level of the hypothalamus, Darpp32 (circles) and TH axons travel in fascicles in control brains. J. In cTKO brains, Darpp32-positive axons are missing (asterisks), and TH-positive axons misroute ventrally (arrows). Dmh- dorsomedial hypothalamic nucleus, Vmh- ventromedial hypothalamic nucleus. 

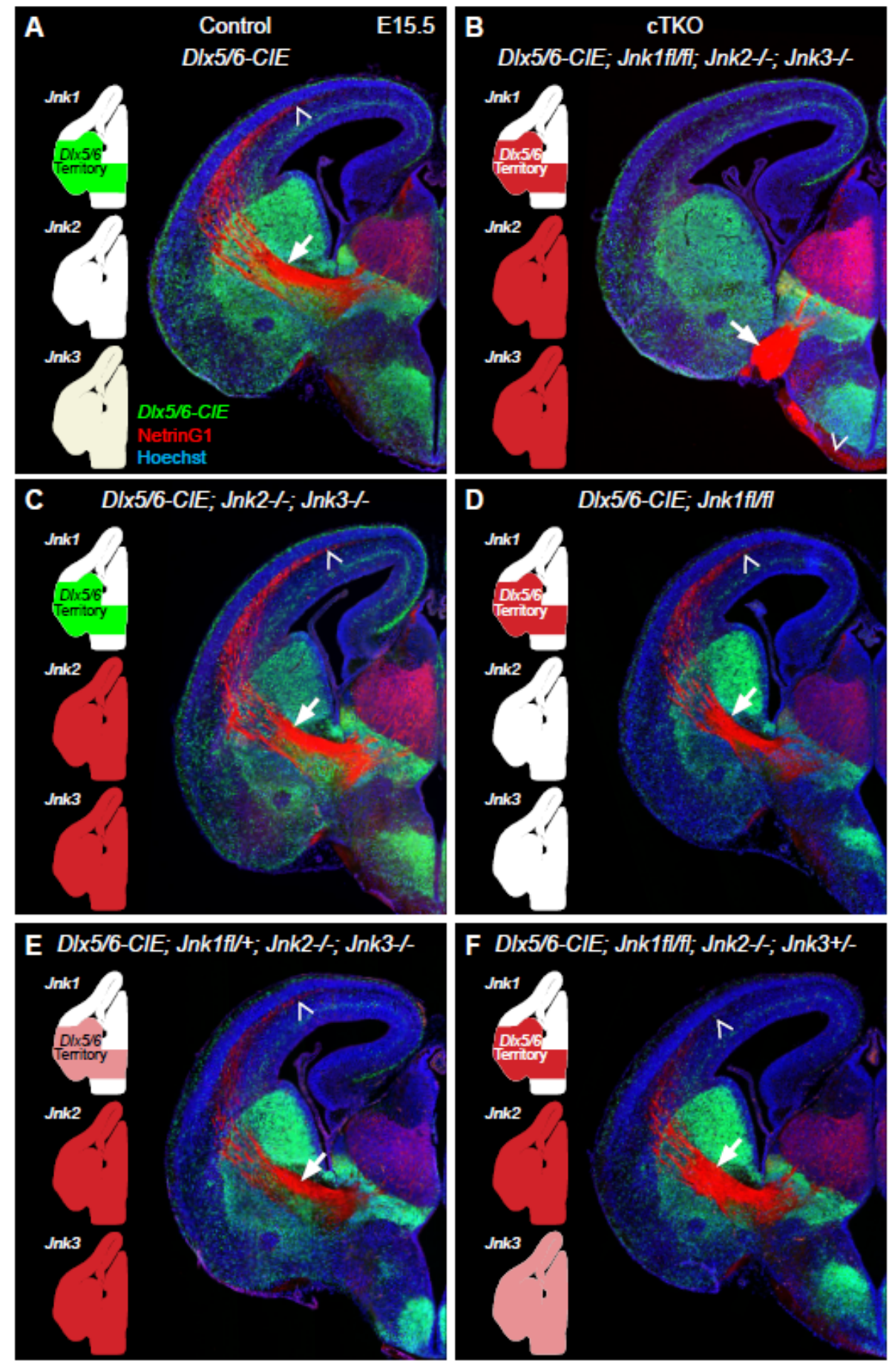

Figure 3.9: Thalamocortical axons require JNK signaling in the territory through which they traverse. A. NetrinG1-labeled thalamocortical axons project through the $D / x 5 / 6$-positive internal capsule (arrow) in route to the cortex (open arrowhead). B. In cTKO brains, NetrinG1postive axons misroute ventrally to the DTB (arrow) and under the hypothalamus (open 
arrowhead). Jnk1 is conditionally deleted with $D / x 5 / 6-C / E$, and Jnk2 and Jnk3 are constitutively removed. C-F. NetrinG1-postive thalamocortical axons project through the internal capsule (arrows) and into the cortex (open arrowheads) in DIx5/6-CIE;Jnk2-/-;Jnk3-/- (C), DIx5/6CIE;Jnk1fl/fI (D), DIx5/6-CIE;Jnk1fl/+;Jnk2-/-;Jnk3-/- (E), and DIx5/6-CIE;Jnk1fl/fl;Jnk2-/-;Jnk3+/(F) mice. Red indicates complete loss of a gene, and pink indicates one copy of the gene is deleted. 

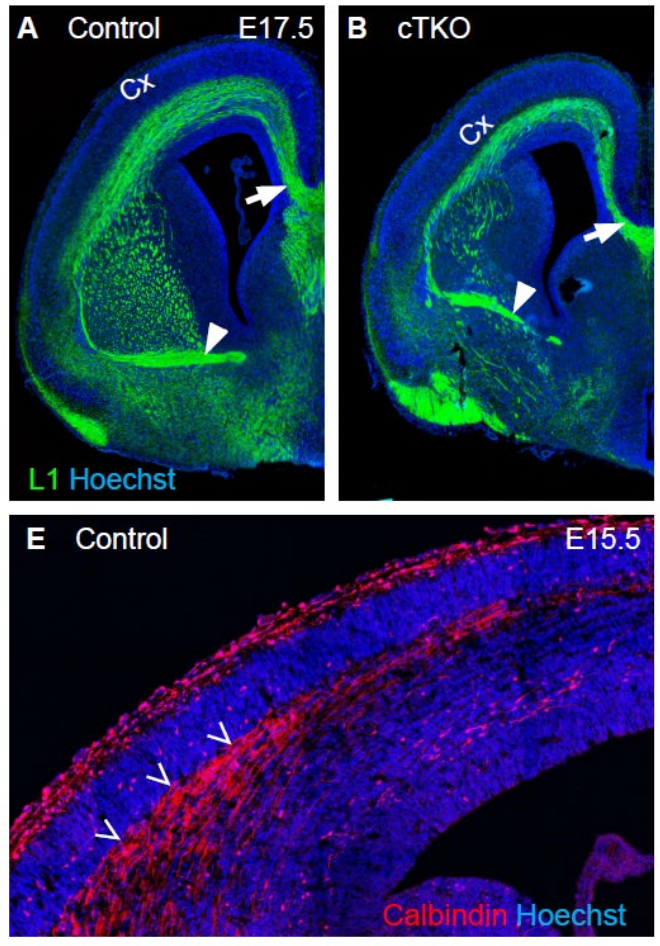
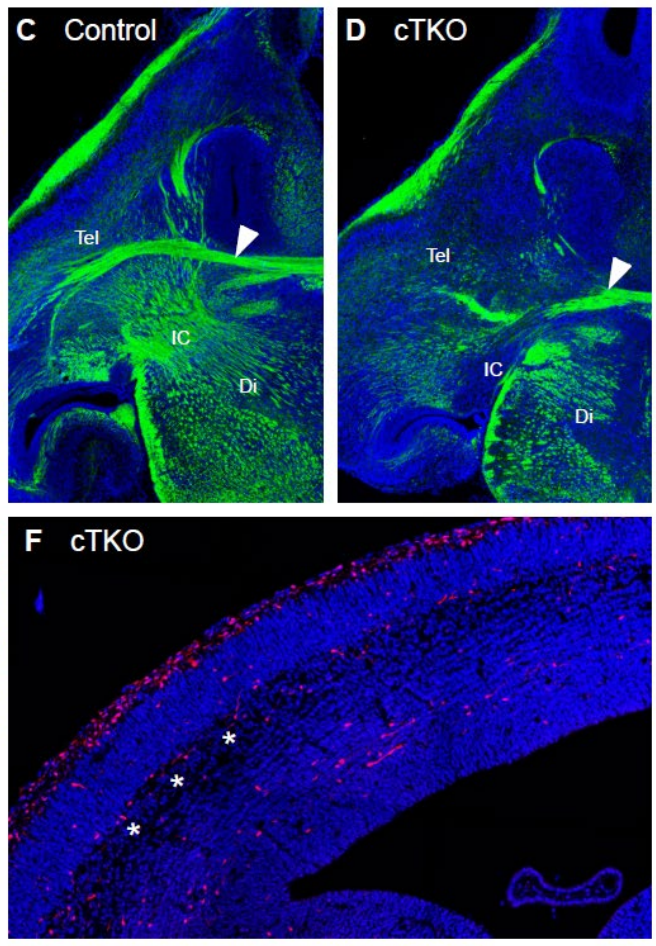

Figure 3.S1: Major axon pathways are disrupted in cTKO brains. A-B. The corpus callosum (arrows) and anterior commissure (closed arrowheads) both cross the midline in control $(\mathrm{A}, \mathrm{C})$ and $c T K O(\mathrm{~B}, \mathrm{D})$ brains. E-F. Immunohistochemistry for Calbindin in E15.5 brain sections labels a population of axons in the cortical wall in control brains (open arrowheads, E), which are missing from cTKO cortices (asterisks, F). 

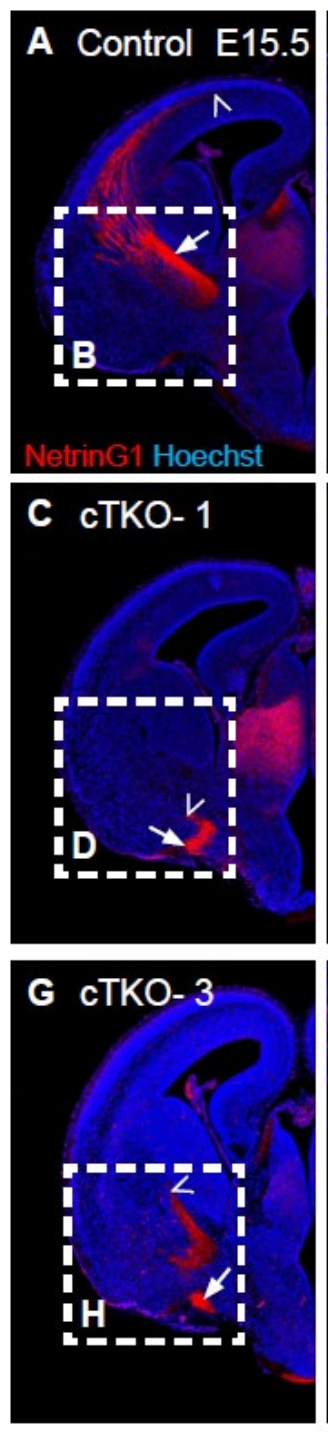
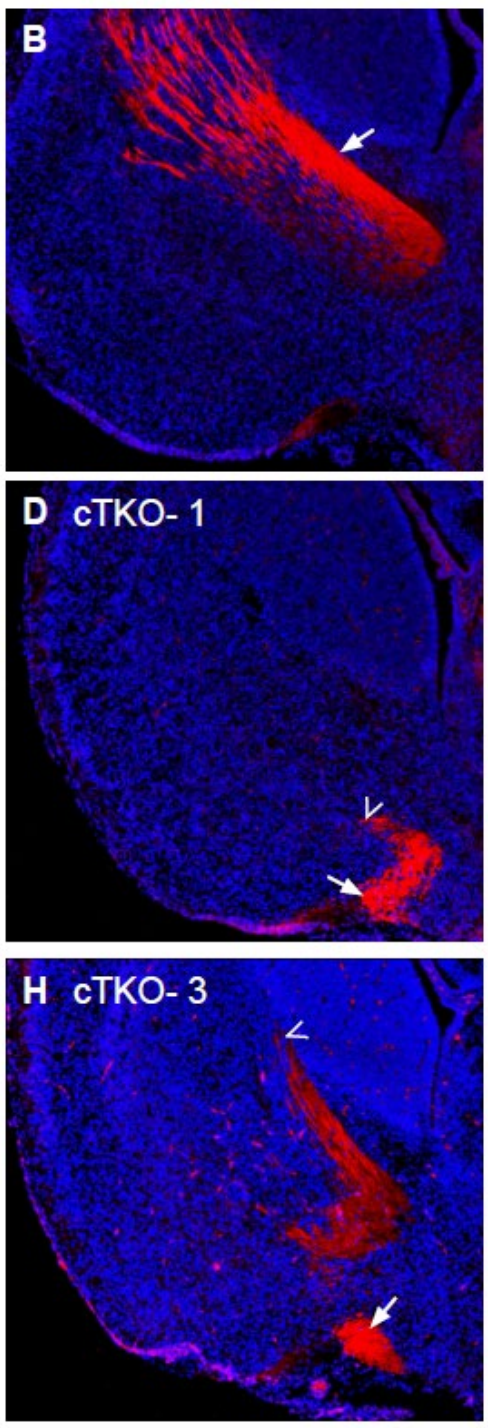

cTKO Spectrum of NetrinG1 Fibers:

- Score 1- no fibers extend from DTB bundle $-n=6 / 15$

- Score 2- stray fibers extend into vTel $-n=5 / 15$

- Score 3- fibers reach Internal Capsule $-n=2 / 15$

- Score 4- some fibers reach cortical ruidment $-n=2 / 15$
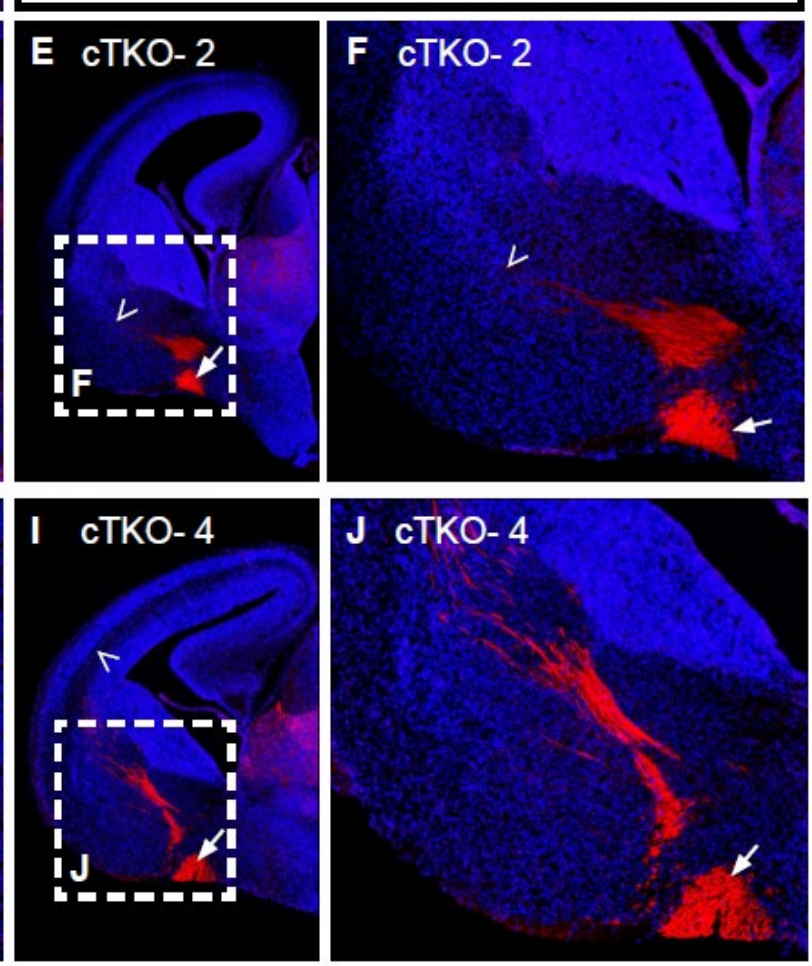

Figure 3.S2: A spectrum of phenotypes is observed in the thalamocortical projections of

cTKO mice. A-B. In control brains, NetrinG1-positive thalamocortical axons project through the internal capsule (arrow) and into the cortex (open arrowhead). C-J. A total of 15 cTKO brains were stained with NetrinG1 along the rostrocaudal axis at E15.5 in vivo to understand the penetrance of the axons projecting into the telencephalon. In all 15 of these brains, axons were present ectopically in the hypothalamus, however the amount that projected into the telencephalon was variable. C-D. In 6/15 cTKO brains (Score 1), no NetrinG1-positive axons extended into the telencephalon from the fascicle at the DTB (arrows, arrowheads). E-F. In 5/15 cTKO brains (Score 2), stray fibers extended into the ventral telencephalon (arrowheads), 
but did not reach the internal capsule. G-H. In 2/15 cTKO brains (Score 3), axons had reached the internal capsule (arrowheads), however did not reach the pallial-subpallial boundary. I-J. In 2/15 cTKO brains (Score 4), some fibers had reached the cortical rudiment (arrowheads), however not to the same extent as controls. 

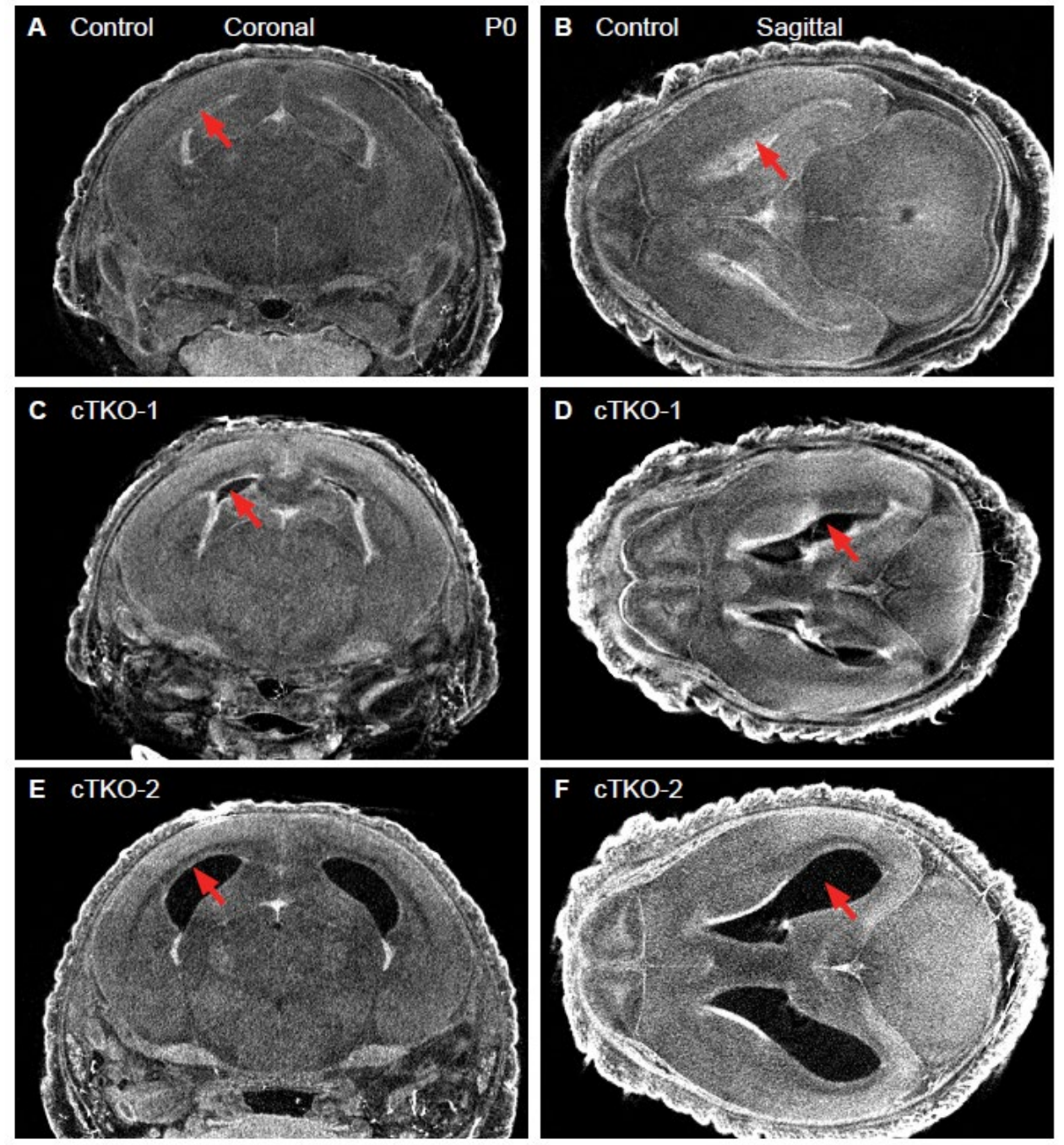

Figure 3.S3: cTKO mice have enlarged ventricular volume at PO. A-B. In control brains in the coronal $(A)$ and sagittal $(B)$ views, very little ventricular volume (arrows) is observed in CT images of whole heads at PO. C-F. Two examples of cTKO brains with increased lateral ventricular volume (arrows). 

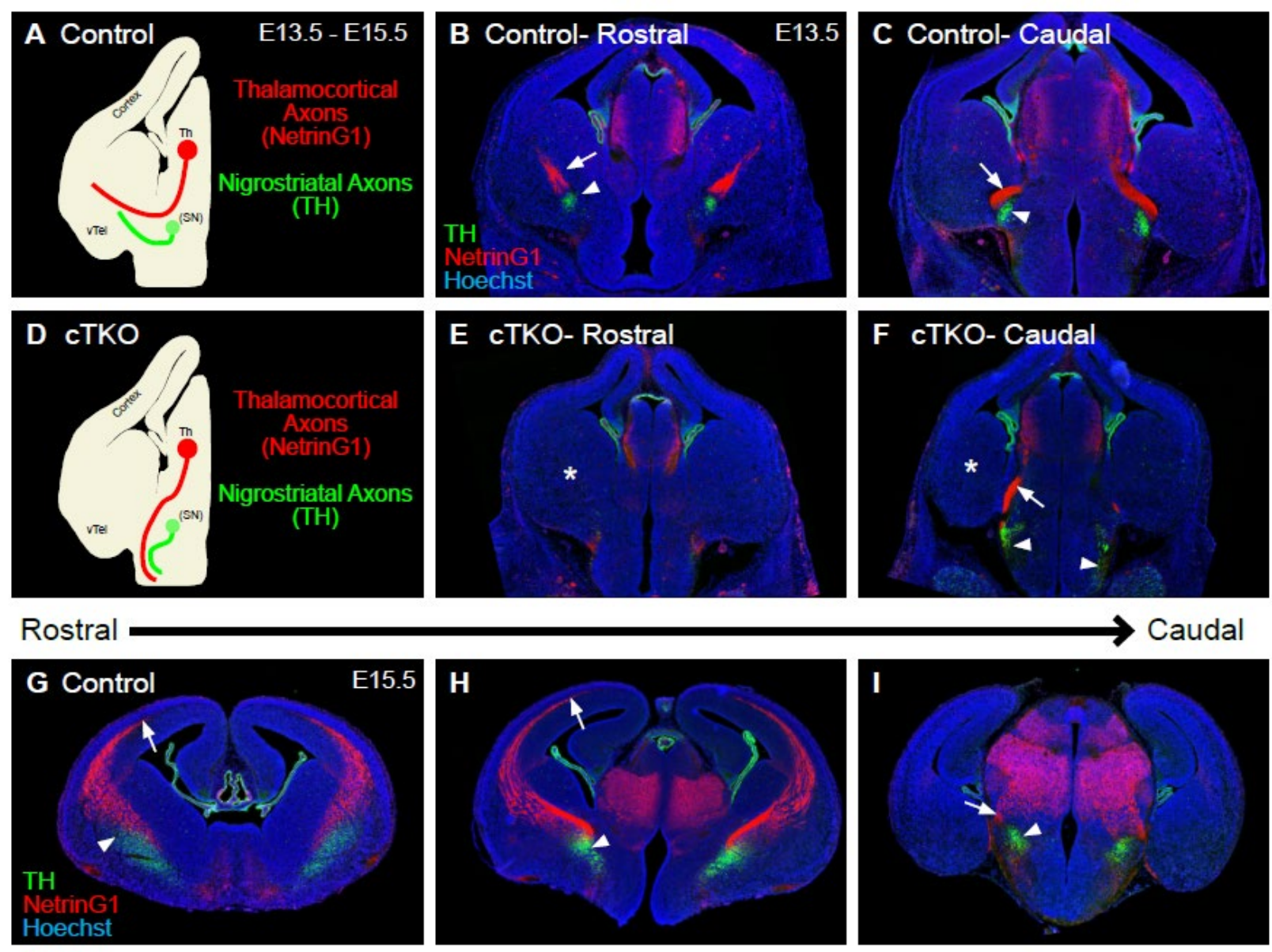

Caudal
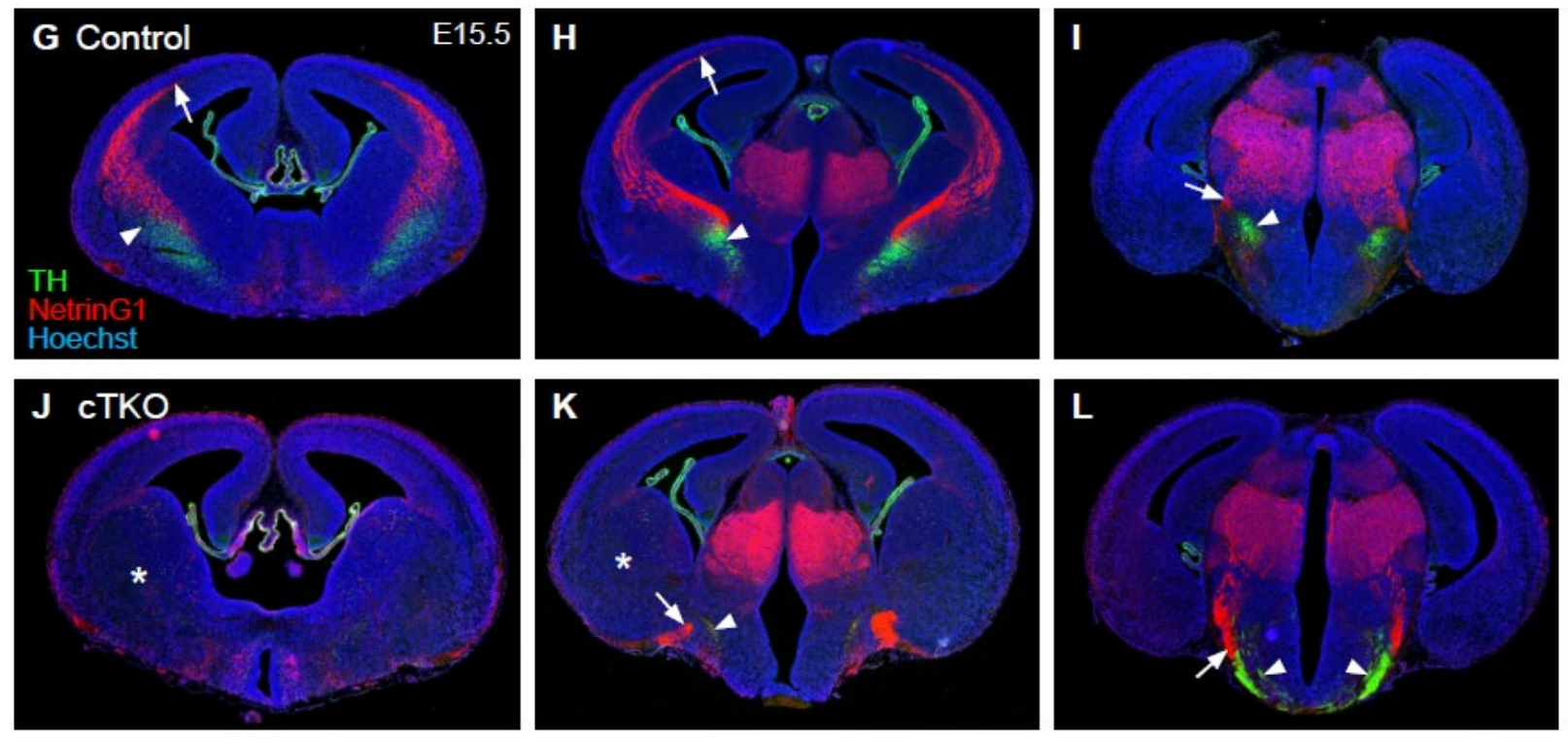

Figure 3.S4: Nigrostriatal axons are misrouted in early development in the cTKO model.

A-C. In control brains at E13.5, NetrinG1-positive thalamocortical axons (arrows) and THpositive nigrostriatal (closed arrowheads) axons have crossed the DTB and entered the telencephalon. D-F. In cTKO brains, both thalamocortical and nigrostriatal axons fail to reach the telencephalon (asterisks). Nigrostriatal axons begin to misroute at E13.5 in cTKO brains (closed arrowhead). G-I. At E15.5, NetrinG1 axons (arrows) have reach the cortex, and TH (closed arrowhead) axons are present in the striatum in control mice. J-L. In cTKO brains, both 
sets of axons fail to reach the striatum (asterisk), and NetrinG1 (arrows) and TH (closed arrowheads) mis-project ventrally. 

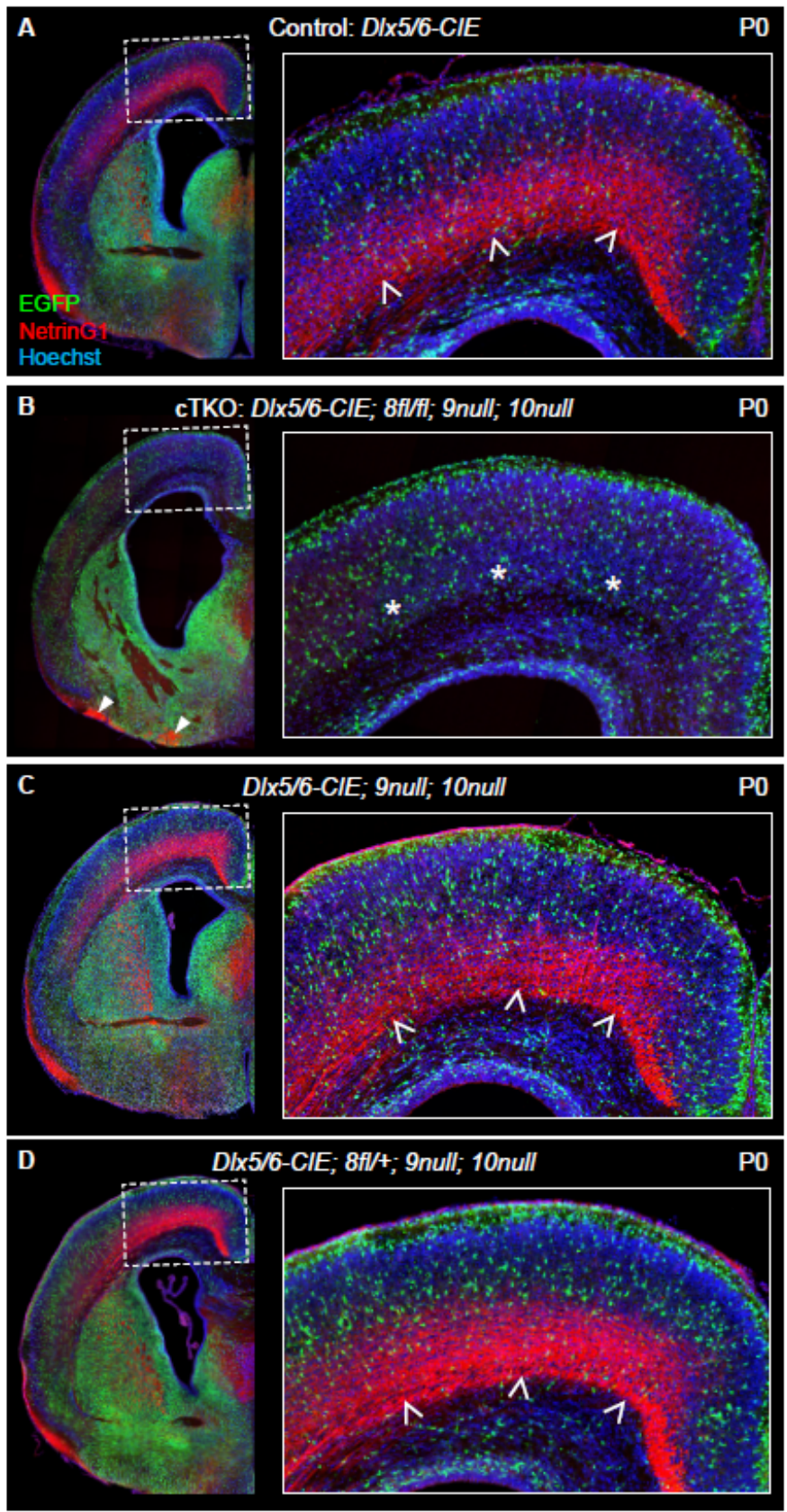

Figure 3.S5: Thalamocortical axons are only missing from the cortices of cTKO brains at

P0. A. In control brains at P0, NetrinG1-positive thalamocortical axons are present in the cortical wall (open arrowheads). B. In cTKO brains, no NetrinG1-postive thalamocortical axons are present in the cortex (asterisks). Ectopic bundles of axons are present in the ventral telencephalon (closed arrowheads). C. In D/x5/6-CIE;Jnk2-/-;Jnk3-/- (C) and DIx5/6- 
CIE;Jnk1fl/+;Jnk2-/-;Jnk3-/- (D) mice, NetrinG1-positive thalamocortical axons are present in the cortical wall (open arrowheads), and no ectopic bundles are located in the telencephalon. 


\begin{tabular}{|c|c|c|c|c|}
\hline Age: & Litters: & Total Animals: & cTKO: & Percent: \\
\hline E12.5 & 4 & 32 & 5 & $15.6 \%(5 / 32)$ \\
\hline E13.5 & 19 & 149 & 19 & $12.8 \%(19 / 149)$ \\
\hline E14.5 & 1 & 8 & 1 & $12.5 \%(1 / 8)$ \\
\hline E15.5 & 31 & 208 & 28 & $13.5 \%(28 / 208)$ \\
\hline E17.5 & 8 & 52 & 10 & $19.2 \%(10 / 52)$ \\
\hline P0 & 33 & 216 & 30 & $13.9 \%(30 / 216)$ \\
\hline P21 & 10 & 43 & 0 & $0.0 \%(0 / 43)$ \\
\hline
\end{tabular}

Table 3.1: cTKO mice are unable to survive past postnatal day 0 . In the cross used to generate triple-knockout mice (cTKOs), the expected Mendelian odds of obtaining the desired genotype is $1 / 8$. Listed are the numbers of litters, total animals, and numbers of $c$ TKO mice at each age point ranging from embryonic $(E)$ day 12.5 through postnatal $(P)$ day 21. Expected Mendelian ratios were collected at all embryonic and early postnatal time points, however no cTKO animals were recovered at P21, indicating early postnatal lethality. 
Chapter 4: Summary and Future Directions 


\section{Summary}

In this thesis, I have uncovered novel autonomous and non-autonomous roles for the cJun N-terminal Kinase (JNK) signaling pathway in early forebrain development. Prior to this work, the roles for JNK in interneuron stream maintenance, cortical allocation of interneurons, and thalamocortical axon pathfinding were unknown. To address these questions, we used a combination of ex vivo pharmacological assays combined with in vivo analyses of genetic knockout mouse models to elucidate JNK's role in these early developmental processes. We created a novel conditional triple knockout (cTKO) mouse model, in which Jnk1 is conditionally removed from DIx5/6 lineage cells in a Jnk2/Jnk3 null background, in order to eliminate all of JNK signaling from DIx5/6 cells. In Chapter 2, we explored the requirement for JNK signaling in the migration and laminar allocation of cortical interneurons, which arise from the $D / x 5 / 6$ lineage, using ex vivo pharmacological inhibition of JNK signaling combined with in vivo characterization of the cTKO model. In Chapter 3, we uncovered a non-autonomous requirement for JNK signaling in early thalamocortical axon pathfinding in the cTKO mouse, where JNK is eliminated in the territory through which these axons traverse. In sum, we discovered JNK signaling to be critical for many different aspects of early forebrain development, through both autonomous and non-autonomous processes.

In Chapter 2, both pharmacological and genetic approaches were utilized to examine the loss of JNK function in cortical inhibitory interneuron development. When the pan-JNK inhibitor SP600125 was applied to cortical slices grown ex vivo, interneurons rapidly departed from preformed migratory streams. Live-imaging data revealed altered cell migration, including reduced speeds, displacements, and straightness of migrating interneurons. In the cTKO mouse model, where all three JNK genes are eliminated from interneurons in vivo, similar interneuron migration phenotypes were observed. After an initial delay into the cortex at embryonic (E) day 13.5, interneurons in the cTKO mouse had advanced to the same medial location in the cortical wall as controls, however they were dispersed from migratory streams, and had altered 
morphologies including more circular cell bodies and shorter leading processes. Live imaging of cTKO cortical slices indicated that interneurons were prematurely departing from migratory streams, which was accompanied by reductions in displacement and straightness of individual migratory cells. With both pharmacological and genetic loss of JNK function, the leading processes of interneurons were oriented away from the tangential plane, indicating the normal transition from tangential to radial migration was impaired. In addition to examining the role of JNK signaling in interneuron stream maintenance, we also examined the role for JNK in early cortical plate allocation. At postnatal $(P)$ day 0 , interneurons in cTKO brains were greatly reduced in the subventricular zone and had accumulated in the lower cortical plate and marginal zone regions. Furthermore, the loss of JNK differentially affected populations of calbindinpositive and calbindin-negative interneurons, indicating that various subtypes of interneurons may have a different requirement for JNK signaling in assuming their correct laminar positions. Through both genetic and pharmacological approaches, we were able to demonstrate that the requirement for JNK signaling was at least in part cell autonomous.

In Chapter 3, we uncovered a novel, non-autonomous requirement for JNK signaling in early axon pathfinding in the developing forebrain. In the cTKO model, a population of axons was missing from the cortical wall at E15.5. Through both immunohistochemical and Dillabeling of different axon populations, the axons missing from the cortex were identified as thalamocortical axons. Instead of coursing through the internal capsule and into the cortex, thalamocortical axons in the cTKO model project ventrally from the thalamus into the hypothalamus, forming an aberrant "U-shaped" bundle of axons. Corticothalamic axons were still present in the cortical wall, as demonstrated through immunohistochemistry and Dil labeling, however they were unable to traverse the internal capsule and reach the thalamus. A developmental time series demonstrated that the misrouting began at E12.5, when thalamocortical axons normally cross the diencephalon-telencephalon boundary (DTB), and persisted until P0. Attempts to rear cTKO mice past P0 proved unsuccessful, indicating early 
postnatal lethality. In the second half of the chapter, cellular and axonal populations known to play a role in the early guidance of thalamocortical axons were examined in the cTKO model. Corridor cells, which are born in the lateral ganglionic eminence and migrate towards the DTB to guide thalamocortical axons, had failed to migrate caudally to the DTB in the cTKO model, and

furthermore ectopically invaded the nascent globus pallidus. A second population of cells called guidepost cells, which normally reside in the ventral telencephalon and project axons back to the thalamus, were severely reduced in cTKO brains. Other axon pathways that also cross the DTB region early in development, including the striatonigral and nigrostriatal pathways, were unable to project past the DTB region in the cTKO model, and therefore failed to innervate their intended targets. Finally, through a series of genetic models, we demonstrated that the requirement for JNK was non-autonomous, and that only the complete loss of JNK function from the territory though which thalamocortical axons traverse resulted in the misrouting phenotypes.

\section{Discussion and Future Directions}

\section{Inhibitory interneurons}

The analysis of interneurons in the cTKO model across embryonic and early postnatal development revealed multiple roles for JNK signaling in cortical inhibitory interneurons. At E13.5, interneurons are delayed in their entry into the cortex in vivo. However, by E15.5, interneurons in the cTKO brain have advanced to a similar location in the medial cortical wall as compared to controls. It is unknown whether the initial delayed cohort of interneurons was able to advance to the medial edge, or whether a second cohort of interneurons was able to compensate for this initial delay. Additionally, at P0, calbindin-positive and calbindin-negative JNK-deficient interneurons were found to differentially allocate in the cortical wall. Therefore, we hypothesized that there may be a different requirement for JNK signaling in interneurons based on their time of generation or subtype. 
The initial cohort of interneurons is generated from the MGE at E9.5, and from the CGE at E12.5 (Miyoshi et al., 2010). The allocation of interneurons in the cortical wall is primarily based on their location of origin, and also their time of birth. MGE-derived interneurons layer in an inside-out pattern based on their time of birth, however CGE-derived interneurons layer in a 3:1 ratio in the upper to lower cortical layers, regardless of their date of birth (Miyoshi and Fishell, 2011). Since the cTKO model used here uses the D/x5/6 Cre driver line, it encompasses most cortical interneurons and does not distinguish between time of origin or subtype. Additionally, the cTKO mouse dies at P0, preventing further analysis of final positioning in the cortical wall as well as subtype specification, which can more effectively be observed at later postnatal time points. Future experiments could be directed at understanding JNK's requirement in different chronologically or subtype-defined populations of interneurons.

\section{Experiments:}

- BrdU is a Thymidine analog used to label a birth-dated cohort of cells, which could be used in both slice culture assays and in vivo models to study JNK's requirement in interneurons born at the same time (Figure A1). Brain slices from BrdU-pulsed dams could be grown in SP600125 to determine if JNK differentially affected the entrance, migration, or allocation of a single cohort of interneurons. Similar analyses could be performed in vivo by studying BrdU-labeled interneurons in the cTKO model.

- Attempts to rear the cTKO mouse past PO have been unsuccessful, which could be due to lethal consequences of the JNK deletion or the need to cross foster these litters. However, using a new genetic approach, mice with the same genotype as the traditional cTKO mouse have survived to P21 and appear phenotypic (Figure A2). cTKO mice generated in this new cross could be analyzed from embryogenesis through early adulthood, which would provide valuable insight into the requirement of JNK in the final laminar allocation and subtype specification of cortical inhibitory interneurons in vivo. 
- Different Cre driver lines can be utilized to study the loss of JNK from different classes of interneurons, such as the Somatostatin-Cre to study loss of JNK from a subtype of MGEderived interneuron, or the Nkx2.1-Cre and Htr3a-Cre to target cells in the MGE and CGE, respectively. These mice may even survive until later postnatal ages, opening the possibility for postnatal anatomical characterization and behavioral assays.

- In our pharmacological approaches to study the loss of JNK function from interneurons, we used the pan-JNK inhibitor SP600125 on ex vivo cortical slices generated from D/x5/6-CIE brains. We could instead use the aforementioned Cre driver lines, crossed to a tdTomato reporter, to examine the pharmacological loss of JNK signaling from specific subtypes of interneurons.

\section{Cortical excitatory neurons and radial glia}

In the cTKO model in vivo, in addition to interneuron phenotypes, disruptions to the underlying cortical plate were also observed at E15.5 (Figures 2.11, A3) and later at P0 (Figure A4). These findings are significant, since patients with autism and epilepsy have been found to have focal cortical dysplasias (Casanova et al., 2013), and genes related to the JNK signaling pathway have been implicated in patients with structural brain malformations (Kariminejad et al., 2011). While a role for JNK signaling in the migration of cortical excitatory neurons has been established by examining the loss of one of the three JNK genes from these neurons (Westerlund et al., 2011; Zhang et al., 2016), the combinatorial loss of more than one JNK gene on the development of cortical excitatory neurons has yet to be explored.

Cortical excitatory neurons are born near the ventricular surface of the cortex between approximately E12.5 and E16.5, migrate towards the pial surface on radial glial scaffolding, and deposit into the developing cortical plate in an inside-out fashion (Noctor et al., 2001). During

this time, cortical interneurons are migrating tangentially through this same region (Marin, 2013), 
and have even been shown to physically contact the same radial glial scaffolding that cortical excitatory neurons use for migration (Yokota et al., 2007). In multiple studies, it has been demonstrated that disruptions to cortical excitatory neurons and even radial glial scaffolding can affect the laminar allocation of cortical inhibitory interneurons (Hevner et al., 2004; Lodato et al., 2011; Lopez-Bendito et al., 2008; Poluch and Juliano, 2007; Talebian et al., 2017), however disruptions in interneuron migration have not yet been demonstrated to affect the organization of other cells in the cortical wall.

In the cTKO mouse, disruptions to cortical excitatory neurons and radial glial scaffolding have been observed in vivo. We hypothesize there could be an autonomous requirement for JNK signaling in cortical excitatory neurons themselves, since both Jnk2 and Jnk3 are constitutively lost in the cTKO model, and other studies have demonstrated a role for JNK in cortical excitatory neuron migration. Alternatively, JNK-deficient interneurons may be nonautonomously influencing the migration of excitatory neurons or the architecture of radial glial scaffolding. Future experiments should expand preliminary findings of disruptions to the underlying architecture of the cortical plate and radial glial scaffolding (Figures A5, A6, A7) to determine JNK's role in the development of these structures, and explore the interactions between JNK-deficient interneurons and cortical excitatory neurons and glia in the developing cortical plate.

\section{Experiments:}

- In vivo analyses of wild type, Jnk2/3-KO, and cTKO mice will enable us to understand if the loss of Jnk2 and Jnk3 impacts the development of the cortical plate, and if this phenotype is exacerbated in the cTKO mouse in which Jnk1 is additionally removed from D/x5/6-lineage cells. P0 cortices can be labeled with different layer markers to examine cortical excitatory neurons (Figures A4, A5), and with Nestin to label radial glia (Figure A7). 
- We can assess JNK's role in early (E12.5) compared to late (E16.5) born excitatory neurons through a dual-pulse Thymidine analog strategy (Figure A6). Later-born cohorts of excitatory neurons must migrate through a cortex containing significantly more interneurons, and if JNK-deficient interneurons could disrupt excitatory neurons, we predict there would be a greater disruption to later-born excitatory neurons in the cTKO model.

- Ex vivo slice culture assays combined with live-imaging techniques can be utilized to examine JNK's role in the dynamic behavior of various cell types in the cortical wall. In DIx5/6-CIE or cTKO brains where interneurons express EGFP, cortical excitatory neurons and radial glia scaffolding can be labeled in red via electroporation, so both cell types can be imaged simultaneously (Figure A7). This study would allow for an assessment of JNK's role in the migration of cortical excitatory neurons themselves, as well as provide insight into dynamic interactions between interneurons and other cortical cells.

- To determine whether JNK-deficient interneurons can non-autonomously influence excitatory neurons in a wild type environment, MGE explants from a cTKO brain can be transplanted onto a wild type cortical slice (Figure A8). After several days, interneurons will have migrated into the cortex, and the layering and architecture of cortical excitatory neurons can be analyzed. If no effect on excitatory neurons is observed, it could be due to the large number of wild type interneurons in the host environment relative to the JNKdeficient transplanted interneurons.

\section{Thalamocortical influence on interneuron migration}

In addition to interactions with cortical excitatory neurons and radial glial scaffolding, it has been demonstrated that interneurons use axons to guide their migration (Denaxa et al., 2001; McManus et al., 2004). Furthermore, in mice lacking thalamocortical input, cortical interneurons are abnormally deposited in the early postnatal cortical wall (Larsen et al., 2019; 
Zechel et al., 2016). In the cTKO mouse, the cortex lacks thalamocortical innervation throughout embryonic and early postnatal development. As a result, JNK-deficient interneurons are migrating in a cortical environment that is missing a key population of axons known to play a role in their laminar allocation. Therefore, we hypothesize that at least some of the interneuron migration phenotypes observed in the cTKO model could be caused by a lack of thalamocortical innervation. Future studies will need to investigate the autonomous versus non-autonomous requirements for JNK signaling in the migration of cortical interneurons.

Several experiments in our laboratory provide evidence supporting an autonomous requirement for JNK signaling in the migration of cortical interneurons. When interneurons are grown in isolation in Matrigel, JNK-inhibited interneurons migrated in slower, shorter, and lessstraight trajectories (Figure 2.9). Other ex vivo studies demonstrated that interneurons from cTKO embryos display altered nucleokinesis and leading process branching when they are grown in a wild type cortical environment (Smith et al., 2020). In the cTKO embryo in vivo, interneurons are delayed into the cortex at E13.5, which is before thalamocortical axons have entered the cortical rudiment, suggesting the delay into the cortex is independent of interneurons' interactions with thalamocortical axons. Future studies can further explore JNK's autonomous roles in interneuron stream maintenance and laminar allocation.

\section{Experiments:}

- An important first experiment is to assess the migration of JNK-deficient interneurons in an in vivo environment containing thalamocortical axons. We could use in utero electroporation of a dominant-negative construct of $\mathrm{JNK}$, or in utero transplantation of a cTKO MGE explant, into the ventral telencephalon of a wild type embryo and allow these JNK-deficient interneurons to migrate in a control environment. We would first need to determine if this manipulation impacted thalamocortical extension into the cortex, since we are asking the question of whether JNK-deficient interneurons can migrate normally 
in the presence of thalamocortical axons. We could then perform analyses similar to the cTKO model, and use in vivo and ex vivo approaches to study the migration and laminar allocation of JNK-deficient interneurons in a wild type environment.

- In preliminary data from our laboratory, when all of JNK signaling is removed from Somatostatin-expressing interneurons in vivo, thalamocortical axons are able to reach the cortex in seemingly normal amounts (Figure A9). Further characterization of this mouse line would enable us to study the migration of a subtype of JNK-deficient interneurons in an environment with thalamocortical axons.

\section{Attractive and repulsive cues for guiding thalamocortical axons}

Our data strongly supports a non-autonomous requirement for JNK signaling in thalamocortical axon pathfinding. Thalamocortical axons are misrouted in the cTKO model when all of JNK signaling is removed from the D/x5/6 territory, which does not target the thalamic neurons themselves. However, the D/x5/6 expression domain is broad, and encompasses many different areas known to be important in thalamocortical axon pathfinding. Therefore, it will be important to decipher where the requirement for JNK signaling is coming from in the cTKO model, which may end up being from more than one region.

Thalamocortical axons must first extend ventrally from the thalamus and through the prethalamus, before making a lateral turn at the diencephalon-telencephalon boundary (DTB; Molnar et al., 2012). This initial crossing of the DTB is aided by repulsion from the hypothalamus and attraction by the ventral telencephalon (Braisted et al., 1999). The repulsive cues from the hypothalamus have been shown to be directed by Slit-mediated repulsion of thalamocortical axons, which express Robo receptors (Bagri et al., 2002; Braisted et al., 2009; Braisted et al., 1999; Lopez-Bendito et al., 2007). The ventral telencephalon exerts attractive properties in the corridor region through gradients of axon guidance molecules such as Netrins 
and Slits (Bielle et al., 2011a; Braisted et al., 2000; Braisted et al., 1999; Leyva-Diaz et al., 2014). JNK's role in mediating Netrin or Slit expression in the thalamocortical axon pathway remains unknown, however JNK has been molecularly linked to both axon guidance molecules in other systems. In commissural neurons from mouse spinal cords, JNK signaling is required for the coordination of Netrin signaling (Qu et al., 2013), and in Drosophila, JNK has been shown to mediate Slit-Robo signaling in epithelial wound repair (lida et al., 2019). It remains to be determined whether Netrin or Slit expression in the ventral forebrain cells or hypothalamus depends on JNK signaling.

In the cTKO model in vivo, thalamocortical axons aberrantly project into the hypothalamus and fail to extend into the ventral telencephalon, and the conditional deletion of JNK in the $D / \times 5 / 6$ territory encompasses both of these regions. Therefore, we hypothesize that the gradients of axon guidance molecules in the hypothalamus and ventral telencephalon have been altered in the cTKO model, and are no longer repulsive or attractive for thalamocortical axons, respectively.

\section{Experiments:}

- In situ hybridization can be used to analyze the mRNA expression of different axon guidance molecules in the developing mouse brain. Slit and Netrin expression can be evaluated at rostral levels in the ventral telencephalon and caudal levels in the hypothalamus in control and cTKO brains to determine if there are any changes to these critical guidance factors.

- In vitro explant assays could be utilized to independently assess the repulsive effects of the hypothalamus and attractive effects of the ventral telencephalon in the cTKO model. A wild type thalamus explant could be grown in close proximity to a cTKO hypothalamus explant, and we could ask whether thalamic axons are repelled by the cTKO hypothalamus. 
Similarly, we could assess whether a control thalamic explant is attracted to a cTKO ventral telencephalic explant.

- Additionally, we can address the question of whether subsets of thalamocortical axons are differentially affected in the cTKO model, since not all axons invaded the hypothalamus in vivo. Calretinin can be used to label a subset of thalamocortical axons originating from the lateral dorsal thalamus (Figure A10). Additionally, two spectrally distinct lipophilic dyes could be placed into separate regions of the thalamus to visualize the trajectories of different thalamic nuclei in the same preparation. These experiments would enable us to begin to assess whether there could be a different requirement for JNK signaling in various populations of thalamocortical axons.

\section{JNK's role in striatal development}

The developing striatum is located in the $D / x 5 / 6$ territory and is therefore directly targeted by our conditional deletion of JNK in the cTKO model. Both striatal axons as well as cells derived from the striatum are known to non-autonomously guide thalamocortical axons in the ventral telencephalon. Striatonigral axons course directly beneath thalamocortical axons at the DTB region, and have reached the substantia nigra by E13.5 (Morello et al., 2015). It has been suggested that striatal axons are critical for laying down a scaffolding for thalamocortical axons, and when striatal axons fail to extend properly, it leads to the misrouting of thalamocortical axons (Morello et al., 2015; Uemura et al., 2007). In addition to striatal axons, "corridor" cells derived from the lateral ganglionic eminence (LGE) migrate towards the DTB region to form a permissive territory for incoming thalamocortical axons (Bielle et al., 2011a; Lopez-Bendito et al., 2006). Disruptions to the corridor region in other mouse models have resulted in the misrouting of thalamocortical axons (Bielle et al., 2011a; Morello et al., 2015; Simpson et al., 2009; Uemura et al., 2007). 
While JNK signaling has not yet been shown to play a role in the development of the striatal cells or axons, JNK is involved in many different aspects of axon growth and neuronal migration (Coffey, 2014; Zdrojewska and Coffey, 2014). Additionally, JNK is molecularly linked to other known regulators of striatal and corridor cell development. JNK is a downstream target in the noncanonical Wnt signaling pathway, which is activated by Wnt ligands binding to Frizzled3 and Celsr3 receptors (Li et al., 2005; Sugimura and Li, 2010). When Frizzled3 or Celsr3 were conditionally removed from the DIx5/6 territory, thalamocortical axons were misrouted in nearly identical patterns to the cTKO mouse (Hua et al., 2014; Qu et al., 2014; Zhou et al., 2008; Zhou et al., 2009), suggesting JNK may act downstream of Frizzled3 or Celsr3 in the noncanonical Wnt signaling pathway.

In the cTKO mouse model, disruptions to both striatal axons and corridor cells have been characterized. Striatal axons in the cTKO model are entangled in the ventral telencephalon and are unable to project caudally through the DTB and reach the substantia nigra (Figure 3.8). Additionally, in the cTKO mouse, corridor cells fail to extend caudally to the DTB at E13.5 (Figure 3.6), and instead invade the nascent globus pallidus. We hypothesize that JNK signaling is autonomously required for the development of the striatum. Deficits in striatal development could then non-autonomously influence the guidance of thalamocortical axons.

Experiments for assessing JNK's role in striatal outgrowth in vitro and in vivo:

- Striatal explants can be grown in Matrigel to examine the outgrowth of axons (Figure A11). Explants from the stratum of wild type and cTKO embryos can be grown in Matrigel to determine if striatal axons are able to extend in vitro. Additionally, wild type explants can be grown in either control or SP600125 media to determine if the pharmacological inhibition of JNK signaling impacts striatal axon outgrowth. 
- It will be critical to examine striatal outgrowth in vivo in the cTKO model at early embryonic time points when major axon pathways are first crossing the DTB. Preliminary data shows that OL-protocadherin-positive striatal fibers are unable to extend in vivo in the cTKO model (Figure A12). Additionally, these fibers express the phosphorylated, active form of JNK (pJNK) in the control brain but not in the cTKO brain, suggesting a critical role for JNK function in the development of the striatum.

Experiments for assessing JNK's role in corridor cell migration:

- Explants from the control and CTKO LGE can be grown in Matrigel and imaged live to evaluate the dynamic migration of corridor cells. The explants can also be grown in proximity to cells expressing Slit, to assess whether JNK-deficient corridor cells can respond to directional guidance cues normally present in the ventral telencephalon.

- A more detailed in vivo analysis of different striatal markers over embryogenesis would provide additional insight into the developmental requirement for JNK signaling in the formation of the striatum (Figures A13, A14). If the positioning of striatal cells near the DTB region was comparable between controls and cTKO mice at later time points, this would suggest that cTKO corridor cells could be delayed in their migration, which would be reminiscent of the delay in migration observed in the cTKO interneurons.

- Corridor cells have been shown to guide thalamocortical axons once they are in the telencephalon, however their role in the initial crossing at the DTB remains largely unknown. Therefore, it is important to assess whether the cTKO corridor region could guide thalamocortical axons in the telencephalon if the axons had crossed the DTB at the appropriate time. We could place a wild type thalamus explant directly into the ventral telencephalon of a cTKO brain slice, and the axon outgrowth towards the cortex can be analyzed. The thalamus explant would need to be fluorescently labeled either with a Dil 
crystal (Figure A15), or genetically with a Cre line that would label thalamocortical axons. We began to try this experiment using the Crh-Cre driver line, which labels thalamocortical axons, however the expression does not come on early enough to assess initial axon extension (Figure A16), however other mouse models that label thalamocortical axons could be explored.

\section{Potential autonomous requirement for JNK in thalamocortical axons}

Even though all of our results strongly suggest a non-autonomous role for JNK signaling in thalamocortical axon pathfinding, it is unknown whether JNK could play an autonomous role in the extension of thalamocortical axons themselves. In the cTKO model, thalamocortical axons are missing Jnk2 and Jnk3, and the role of JNK signaling in thalamocortical axon extension has not been explored. Even though thalamocortical axons are able to reach the cortex in the DIx5/6-CIE; Jnk2-/-; Jnk3-/- mouse, and even in the DIx5/6-CIE; Jnk1fl/+; Jnk2-/-; Jnk3-/- mouse, it is unknown whether the axons reach the cortex in the correct number and position. Additionally, we cannot rule out the possibility the misrouting phenotypes in the cTKO model comes from a combinatorial loss of all of JNK signaling from the $D / \times 5 / 6$ territory, combined with the loss of Jnk2 and Jnk3 from the axons (and the rest of the mouse).

Although JNK's role in thalamocortical axon pathfinding has not been established, JNK has been shown to play a role in axon growth and generation (Arthur-Farraj et al., 2012; Oliva et al., 2006; Tararuk et al., 2006), and disruptions to the upstream activator of JNK signaling, MKK7, have been shown to disrupt the internal capsule early in development (Yamasaki et al., 2011). We therefore hypothesized that JNK is autonomously required in the extension and guidance of thalamocortical axons.

Experiments: 
- Thalamic explants can be grown in Matrigel in control and JNK inhibitor to determine if complete loss of JNK function affected thalamic axon outgrowth. This could also be performed using thalamus explants from JNK knockout mice, including single and combinatorial deletions of JNK, to assess if different JNK genes uniquely contribute to thalamic outgrowth.

- To understand whether JNK is developmentally regulated in the thalamus, we can use in situ hybridization to analyze the expression of each JNK gene in thalamic nuclei at different developmental time points. This experiment would first be done in control brains to understand the expression over time, and could be expanded to include different JNK knockout models including the cTKO mouse, to determine if any compensation was taking place.

- The use of a new in vivo conditional genetic knockout mouse model would enable us to eliminate all of JNK signaling from the thalamus itself. A Cre driver line such as Gbx2, which is expressed in the developing thalamus, could be utilized to determine if the loss of JNK from the thalamus impacted thalamocortical axon growth and guidance.

\section{The possible influence of glial cells on thalamocortical axon pathfinding}

The two main types of cells in the nervous system are neurons and glial cells, and while the roles of neurons in thalamocortical axon pathfinding have been extensively studied, the potential role of glial cells in the guidance of thalamocortical axons remains largely unknown. Glial cells outnumber neurons, and in the central nervous system can roughly be divided into oligodendrocytes and astrocytes, which provide a supportive framework for the development of the rest of the nervous system (Jessen, 2004). While glial cells are known for their roles in the myelination and support of axons, they also physically interact with both neurons and axonal growth cones to provide critical guidance in the developing brain (Nave, 2010; Rigby et al., 
2020). The classic example of a neuron-glia interaction in the developing forebrain is the glialguided radial migration of cortical excitatory neurons along radial glial scaffolding in the cortical wall (Rakic, 1972; Tan and Shi, 2013). Later studies demonstrated that cortical inhibitory neurons also physically contact radial glia during their migration in the cortical wall (Yokota et al., 2007), and disruptions to radial glial scaffolding altered the migration and laminar allocation of cortical interneurons (Poluch and Juliano, 2007; Talebian et al., 2017).

In addition to their known roles in cell-cell interactions, glia play critical roles in axon guidance both directly through contacting the growth cones of axons or indirectly through the secretion of signaling molecules or other factors that alter the local environment (Chotard and Salecker, 2004; Rigby et al., 2020). Glial cells located along the midline provide guidance for corpus callosum development, with glial cells located directly below the callosal axons in the glial wedge, cells located above the callosal axons within the indusium griseum, and glial cells located in the midline zipper and glial sling (Shu et al., 2003; Shu and Richards, 2001; Silver et al., 1982). Midline glia are also important for other commissural axon tracts including the optic chiasm (Marcus et al., 1995) and anterior commissure (Cummings et al., 1997). The secretion of both long- and short- range guidance molecules by glial cells located along the midline serve to guide callosal axons, some of which include Slits, Netrins, and Semaphorins (Nishikimi et al., 2013). These same guidance molecules are critical in the early pathfinding of thalamocortical axons, however the role of glia in the early guidance of thalamocortical axons remains largely unexplored.

Two separate studies explored the role for glial cells in thalamocortical axon pathfinding within the diencephalon. The transcription factor Olig2, which is required for the proper development of oligodendrocytes, is expressed in the embryonic prethalamus, and loss of Olig2 leads to hypoplasia of the prethalamus, expansion of the thalamic eminence, and delayed extension of thalamocortical axons (Ono et al., 2014). Within the thalamus itself, disruptions to 
radial glial cells resulted in the mis-projection of thalamocortical axons (Botella-Lopez et al., 2019).

The location of glial cells within the ventral telencephalon, along with their known roles in axon guidance, places them in a position to have a potential influence as guidepost cells in early thalamocortical axon pathfinding. Oligodendrocyte precursor cells are present in the ventral telencephalon from the medial ganglionic eminence to the anterior entopeduncular area around embryonic day 12 , which is when thalamocortical axons first cross the diencephalontelencephalon boundary (Naruse et al., 2017). Microglia from outside the CNS are known to invade the brain parenchyma, and cluster near developing axons throughout embryonic development at timepoints that coincide with thalamocortical axon extension through the ventral telencephalon and into the cortex (Pont-Lezica et al., 2014; Reemst et al., 2016; Squarzoni et al., 2014). Indeed, microglia regulate the outgrowth of dopaminergic axons in the ventral telencephalon, which follow a similar trajectory as thalamocortical axons across the diencephalon-telencephalon boundary and into the telencephalon (Squarzoni et al., 2014).

Radial glia line the ventricular zone (VZ) of the ventral telencephalon, and have a bipolar morphology with an apical process at the $\mathrm{VZ}$, and a basal process extending beyond the $\mathrm{VZ}$ (García and Harwell, 2017). They give rise to all neurons and glia generated in the ventral telencephalon, either directly or indirectly through generation of other progenitor cell types (Marín and Müller, 2014). Thus, radial glia are the precursors for many critical cell types involved in the guidance of thalamocortical axons through the ventral telencephalon. In addition to their role as a precursor, radial glia are known to form fascicles at different boundary regions, including the pallial-subpallial boundary in the telencephalon and along rhombomere boundaries in the developing hindbrain (Neyt et al., 1997; Yoshida and Colman, 2000). Very little is known about radial glia extending across the developing diencephalon-telencephalon boundary, however in the chick embryo, radial glial processes from the thalamic eminence in the diencephalon were found to extend towards the amygdala in the telencephalon (Trujillo et al., 
2005). Overall, the direct role of glia in thalamocortical axon pathfinding remains largely unexplored, however given their positioning in the ventral telencephalon along with their known roles in axon guidance, glia have the potential to influence thalamocortical axons from very early in development.

\section{Final Thoughts}

The cerebral cortex is able to execute a wide variety of different tasks due to its intricate cellular architecture, combined with its extensive connections to different regions of the brain. This highly organized structure is formed over a large portion of embryonic development, which persists into early postnatal life. Disruptions at any point during this process can lead to lasting changes and result in many different neurodevelopmental disorders. Therefore, it is critical to understand the developmental mechanisms that regulate not only the formation of each cell type or structure, but the creation of the brain as a whole.

Several studies have demonstrated a role for JNK in neurodevelopmental disorders. Patients with known mutations in JNK genes or the JNK signaling pathway have presented with autism, intellectual disability, epilepsy, and schizophrenia, and largescale genetic analyses have suggested that JNK is likely to play a role in the etiology of brain malformations. However, it is unknown how disruptions to the JNK pathway can cause neurological diseases. Here, we provide evidence supporting various roles for JNK in the development of the mouse forebrain including the migration and allocation of cortical interneurons, as well as early pathfinding of thalamocortical axons. This information could be used to help guide studies aimed to understand underlying neurobiological dysfunction in patients with known genetic mutations. For instance, brain organoids can be grown from a patient's skin cells, and various different cell types can be analyzed. Further, employing more sophisticated methods such as the liveimaging techniques utilized here, questions about the dynamic behavior of cells from a patient 
with a disorder can now be studied. With the novel finding of JNK's role in thalamocortical axon pathfinding, this may help direct a patient's treatment plan who has a known genetic mutation in the JNK signaling pathway. A patient with generalized intellectual disability, for instance, may now undergo a fMRI to specifically look for functional impairment in thalamic connectivity, which could help change the course of his or her treatment plan. While a patient is highly unlikely to present with the same genetic mutation as the cTKO mouse, using conditional knockout approaches allows us to study the role of genes in specific cell types that are otherwise lethal when mutated in all tissue, such as the JNK genes.

Even though advances have been made in understanding the developmental regulation of individual cell types, it is important to remember that nothing develops in isolation. Changes to one population of cells or brain region can indirectly, or non-autonomously, influence the development and final organization of other brain regions. In this thesis, I explored both autonomous and non-autonomous requirements for the c-Jun N-terminal Kinase (JNK) signaling pathway in forebrain development. In Chapter 2, I discovered roles for JNK signaling in the migration and allocation of cortical inhibitory interneurons. In Chapter 3, I uncovered a novel, non-autonomous requirement for JNK signaling in thalamocortical axon pathfinding. In the future, it will be critical to gain insight into not only the autonomous requirements of different signaling pathways in development, but also to understand how they can non-autonomously impact other structures. 
Appendix 

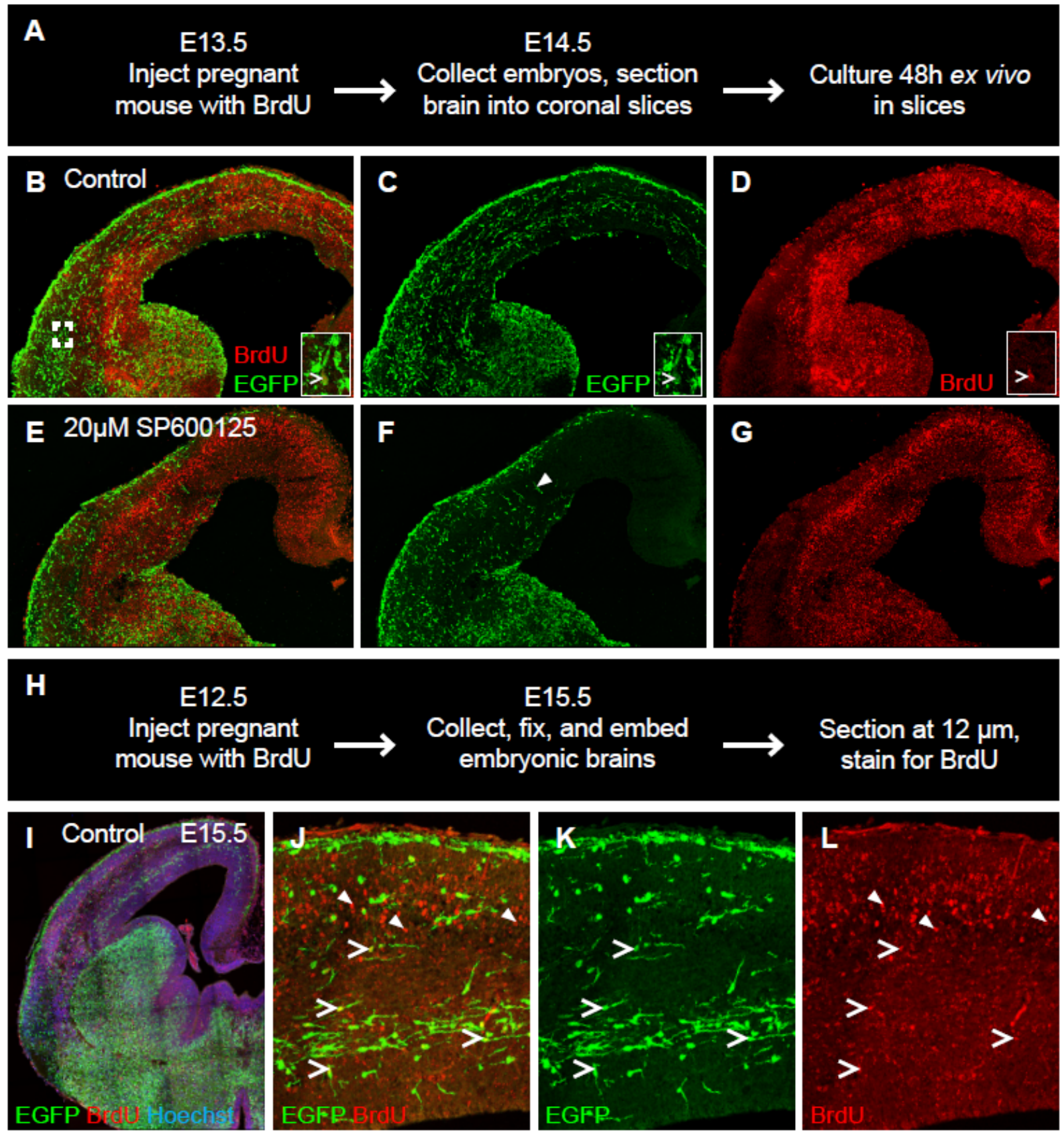

Figure A1. BrdU can be used to label a birth-dated cohort of neurons in the developing

mouse brain. A. Timed-pregnant dams are injected with $50 \mathrm{mg} / \mathrm{kg} \mathrm{BrdU}$ at E13.5. D/x5/6-CIE positive embryonic brains are harvested at E14.5, sectioned coronally at $350 \mu \mathrm{m}$, then cultured 48 hours ex vivo in control (B-D) or $20 \mu \mathrm{M}$ SP600125 JNK inhibitor (E-G). B-G. After 48 hours in culture, brain slices are embedded, re-sectioned into $12 \mu \mathrm{m}$ thin sections, and labeled for 
EGFP and BrdU. Interneurons co-labled with EGFP and BrdU (inset, B-D) were generated at the time of the BrdU pulse. In JNK-inhibited conditions, interneurons are delayed in their entry into the cortex (E-F), and many are radially-aligned (closed arrowhead, F). H. Timed-pregnant dams are injected with BrdU at E12.5. DIx5/6-CIE positive embryonic brains are harvested at E15.5. I-L. Immunohistochemistry for EGFP and BrdU identifies interneurons (open arrowheads) and excitatory neurons (closed arrowheads) that were generated at the time of the BrdU pulse. 


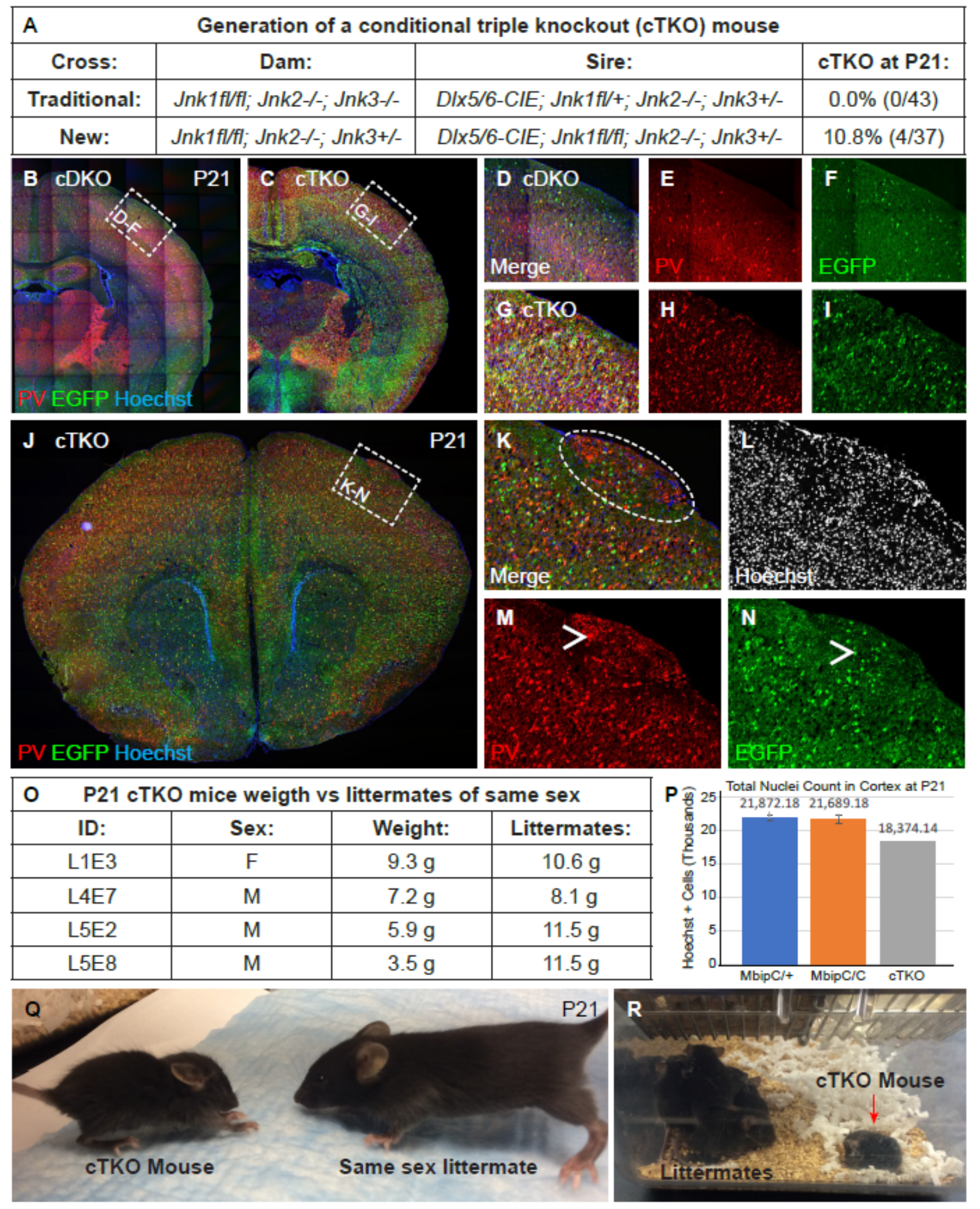

Figure A2. cTKO mice generated through a different genetic cross can survive until P21, however display physical and histological phenotypes. A. Different genetic approaches to achieve the cTKO genotype D/x5/6-CIE; Jnk1fl/fl; Jnk2-/-; Jnk3-/-. In the traditional cross, no 
pups survived until P21, however in the new cross, 4 pups survived until P21 in seemingly normal Mendelian ratios. B-I. Comparison of parvalbumin and EGFP-positive interneurons at P21 in cDKO (DIx5/6-CIE; Jnk1fl/fl; Jnk2-/-) and cTKO brains reveals an apparent increase in the number of interneurons in the cTKO brain. $\mathbf{J}-\mathbf{N}$. A cortical malformation is present in a cTKO brain at P21, which contains clusters of parvalbumin (open arrowhead, M) and EGFP (open arrowhead, $\mathrm{N}$ ) positive interneurons. $\mathrm{O}$. cTKO mice that survived until P21 weighed less than their same-sex littermates. P. Total nuclei counts of cortical cells in sections at the level of the anterior commissure at P21, compared to Nkx2.1Cre; MbipC/+ and Nkx2.1Cre; MbipC/C mice. $\mathrm{n}=1$ for the cTKO data. Q-R. A cTKO mouse at P21 was greatly reduced in size compared to a same-sex littermate, and spent the majority of time being socially distant. 


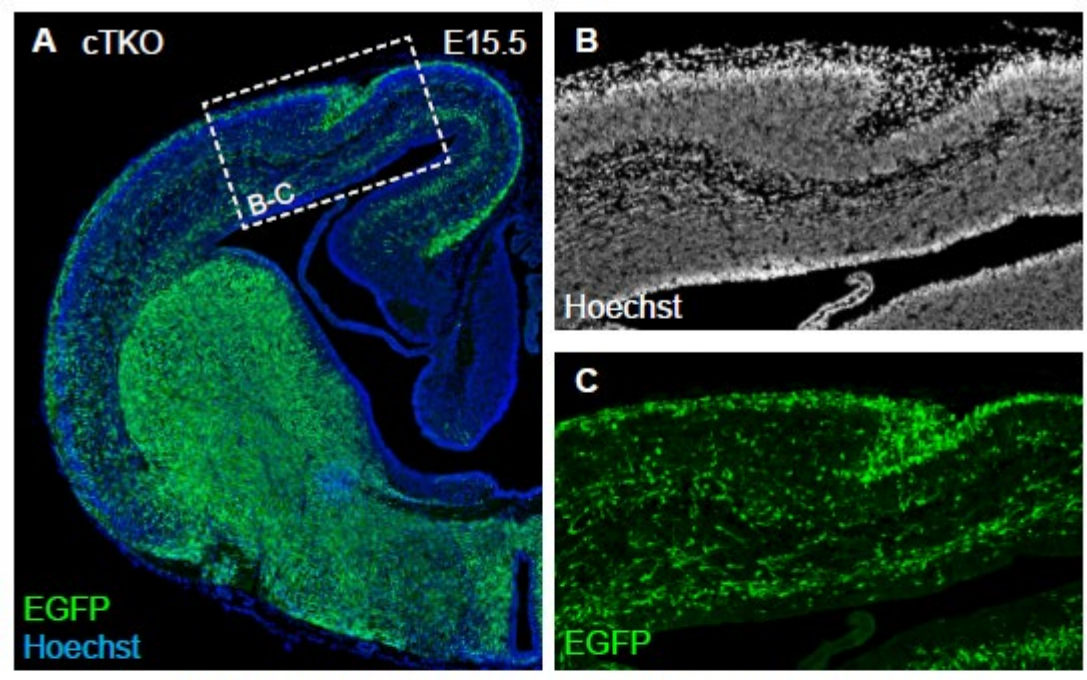

Malformation emerging in series Rostral (D) to Caudal (I)
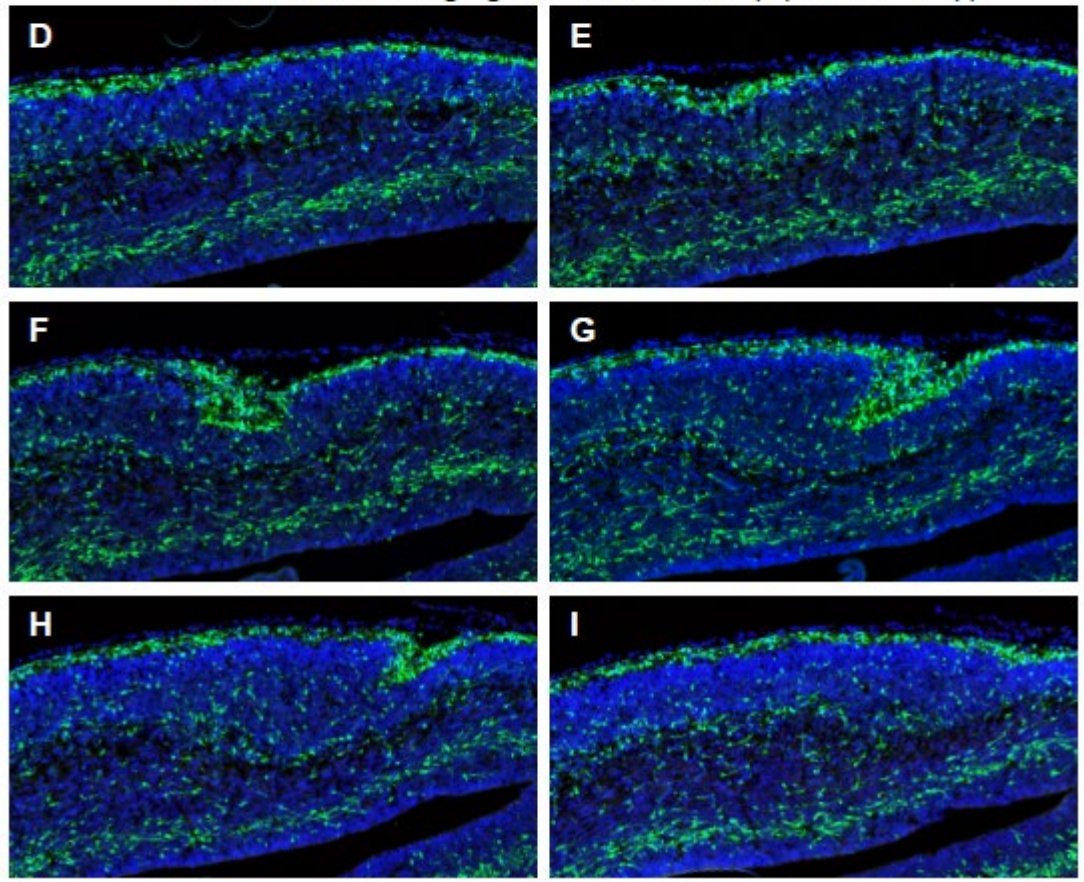

Figure A3. Malformations are present in cTKO brains at E15.5 and contain disruptions to interneurons and the developing cortical plate. A. A malformation is present in the midcortical wall of an E15.5 cTKO embryo. B. The developing cortical plate is grossly altered, and the pial surface remains largely intact. C. Interneurons accumulate in the region that is malformed. D-I. Sections collected $36 \mu \mathrm{m}$ apart show the malformation along the rostrocaudal axis. 

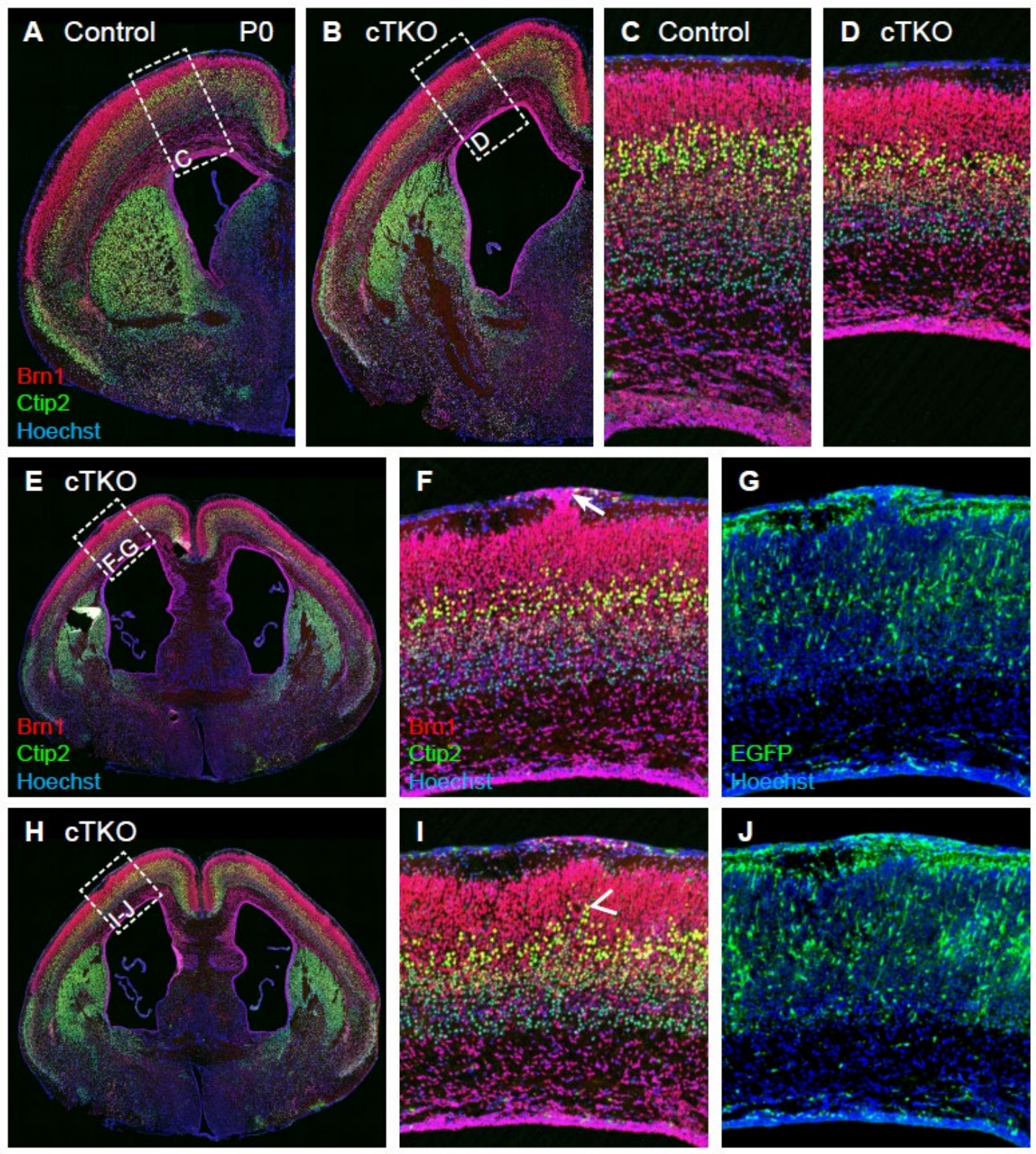

Figure A4. The allocation of cortical excitatory neurons and inhibitory interneurons is

disrupted in cTKO cortices at PO. A-D. At PO in both control and cTKO brains, Brn1 labels the majority of cortical excitatory neurons, and Ctip2 labels lower-layer neurons. Compared to control cortices $(A, C)$, the cortical wall is much thinner in cTKO mice, and cortical layers appear more compact $(B, D)$. E-J. A disruption to the organization of both Brn1 (arrow, F) and Ctip2 (open arrowhead, I) excitatory neurons is identified in the cTKO cortex at P0. Interneurons are also disrupted in these areas of cortical malformations $(\mathrm{G}, \mathrm{J})$. 

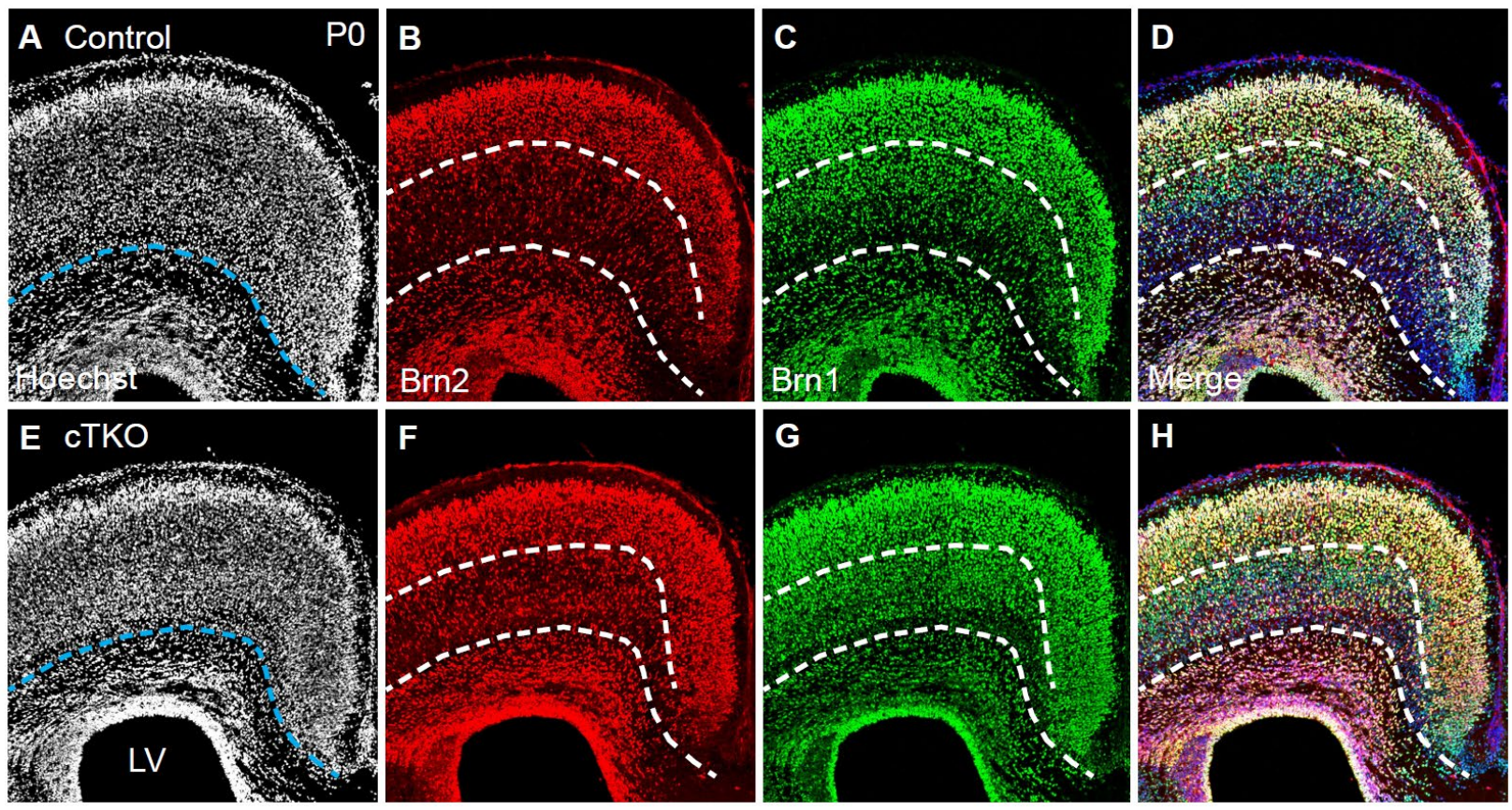

Figure A5. The organization of the developing cortical plate is altered in cTKO mice at P0.

A-D. In control cortices, Brn2 labels upper-level excitatory neurons, and Brn1 labels the majority of excitatory neurons. The blue dashed line represents the bottom of the developing cortical plate. E. In the cTKO brain, the cortical wall is noticeably thinner, and the lateral ventricle (LV) is increased in size. F. Brn2-positive neurons occupy a larger portion of the developing cortical plate. G-H. Brn1-positive neurons are more evenly distributed throughout the cortical wall in cTKO mice, compared to the higher density of Brn1 neurons in upper layers in control brains (C). 

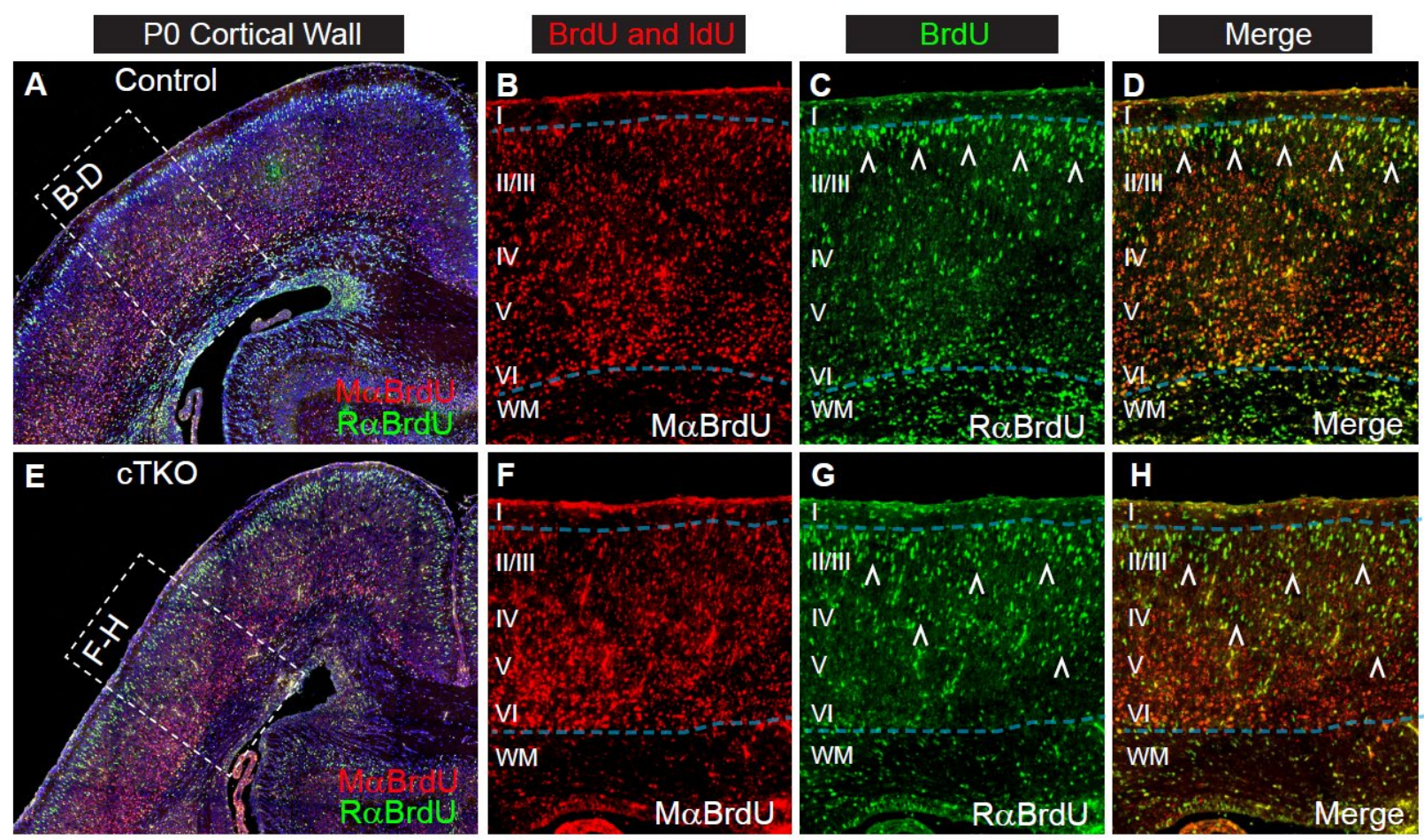

Figure A6. Early- and late-born cohorts of cortical excitatory neurons are uniquely

identified in the early postnatal cortical wall. A-D. Timed-pregnant dams were injected with IdU at E12.5 and BrdU at E16.5. Pups from the dams were perfused at P0, and brains were collected for analyses. Mouse anti-BrdU labels both BrdU and IdU (B), and rat anti-BrdU labels only BrdU (C). Therefore, early-born cells at E12.5 which incorporated IdU are Mouse antiBrdU positive, but Rat anti-BrdU negative. Late-born cells at E16.5 which incorporated BrdU are mouse and rat anti-BrdU positive, and are organized in the upper cortical plate in control brains (open arrowheads, C-D). E-H. In cTKO brains at P0, late-born excitatory neurons are more dispersed throughout the cortical wall (open arrowheads, G-H). 

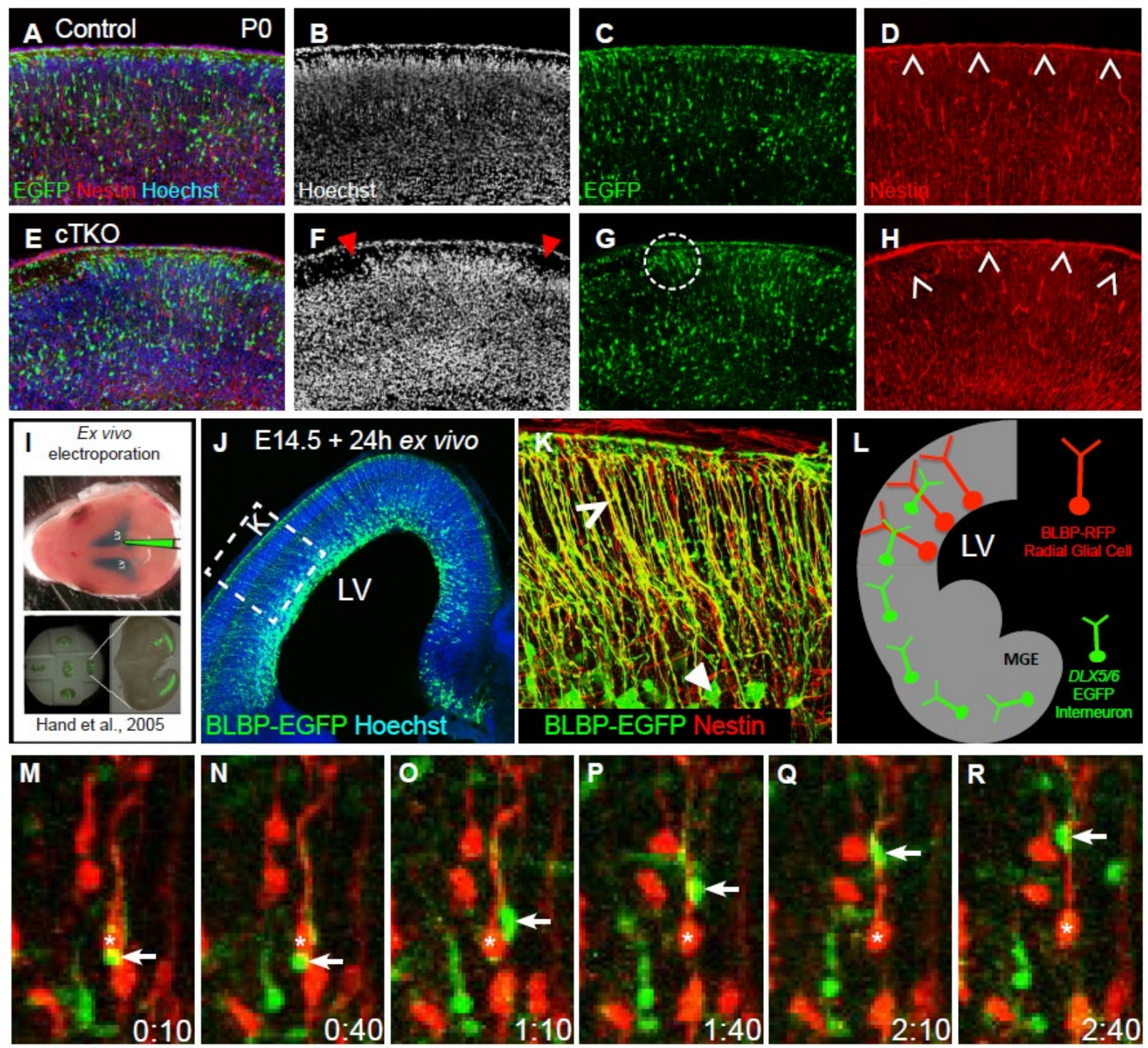

Figure A7. Radial glia are disrupted in the cTKO cortical wall and can be analyzed using ex vivo slice culture assays. A-D. P0 control DIx5/6-CIE brain labeled with Hoechst as a nuclear counterstain (B), EGFP which labels interneurons (C), and Nestin revealing radial glial fibers that extend a process to the pial surface (open arrowheads, D). E-H. In P0 cTKO brains, the cortical plate is inundated at two locations (red closed arrowheads, F), interneurons have formed clusters (circled, G), and radial glial fibers are detached from the pial surface (open arrowheads, H). I. Experimental design adapted from (Hand et al., 2005), in which the lateral ventricle is electroporated with BLBP to label radial glial fibers. J. Brain slice grown for 24 hours 
ex vivo after electroporation with BLBP reveals radial glial cells with cell bodies located at the lateral ventricle, and processes that extend to the pial surface. K. Immunohistochemistry of radial glia cells from electroporated slices labeled with BLBP-EGFP and Nestin shows cell bodies (closed arrowhead) and processes (open arrowhead). L. Experimental design for using BLBP-RFP to label the radial glia in red, in slices where interneurons are labeled with EGFP from DIx5/6-CIE mice. M-R. Live imaging of DIx5/6-CIE brain slices electroporated with BLBPRFP. An interneuron labeled in green (arrow) migrates along radial glia in red (asterisk). 

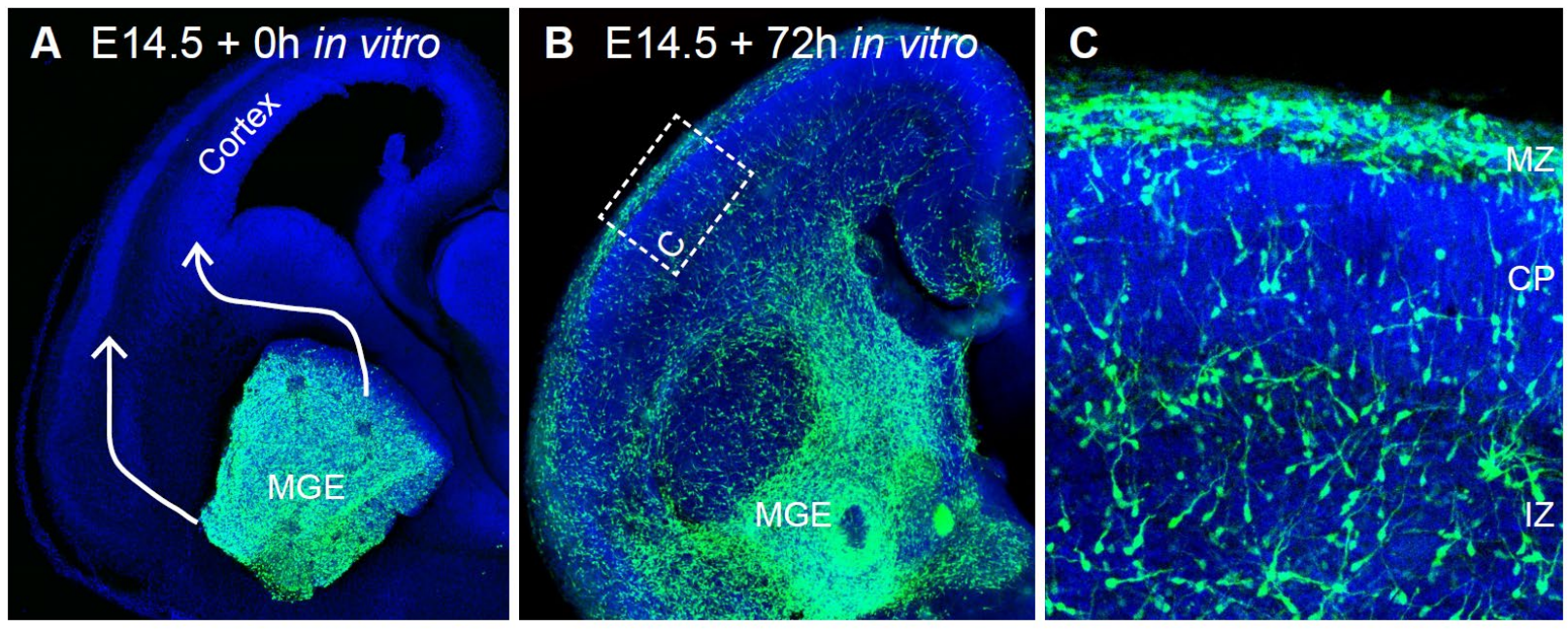

Figure A8. Medial ganglionic eminence transplants contain interneurons that migrate into the cortex of recipient forebrain slices. A. E14.5 medial ganglionic eminence (MGE) explants were generated from DIx5/6-CIE positive mice and were placed onto the ventral forebrain of DIx5/6-CIE negative embryos. B. After 72 hours in vitro, interneurons have migrated through the telencephalon and into the cortex of host brain slices. C. Interneurons are present throughout the cortical wall in the marginal zone $(M Z)$, cortical plate $(C P)$, and intermediate zone $(I Z)$. 

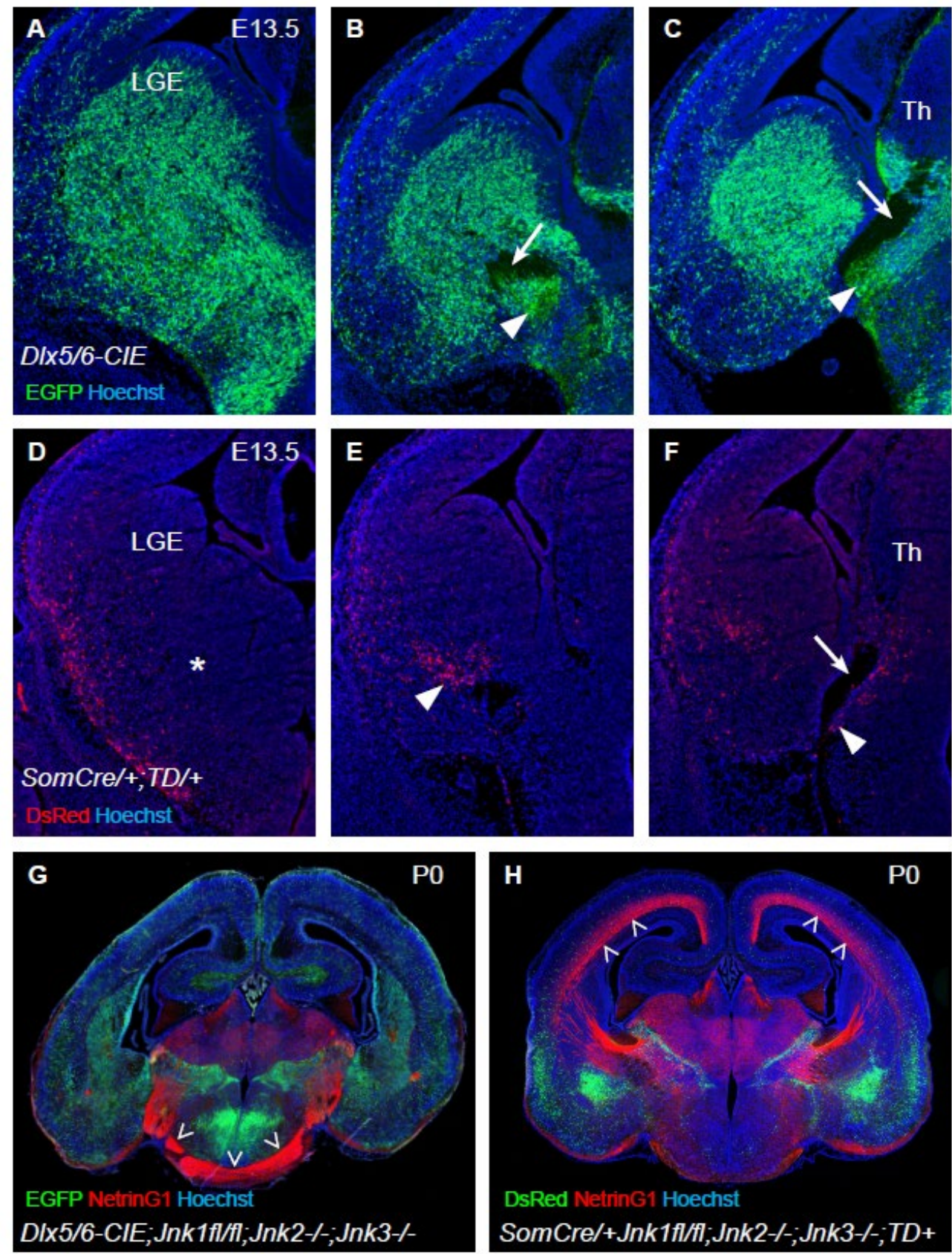

Figure A9. Genetic loss of all three JNK genes from the DIx5/6, but not the Somatostatin, territory results in thalamocortical axon misrouting phenotypes. A-C. $D / \times 5 / 6-C / E$-labelled cells and axons in an E13.5 control mouse brain from rostral (A) to caudal (C) locations. EGFPpositive cells span the ventral telencephalon that thalamocortical axons (arrows, B-C) traverse in early development, including the territory right beneath the axons at the DTB region (closed arrowhead B,C). D-F. SomatostatinCre-positive cells at E13.5 are more sparsely labeled, and 
do not span the majority of the ventral telencephalon (asterisk, D). Cells are located along the lateral ventral telencephalon (D), just lateral to the incoming thalamocortical axons (closed arrowhead, E), and are sparsely located at the DTB (closed arrowhead, F) beneath the thalamocortical axons (arrow, F). G. Loss of all three JNK genes from the DIx5/6 territory results in a massive ventral misrouting of thalamocortical axons beneath the hypothalamus in cTKO brains at PO (open arrowheads). H. When all three JNK genes are removed using SomatostatinCre, NetrinG1-positive thalamocortical axons have reached the cortex at P0 (open arrowheads). 

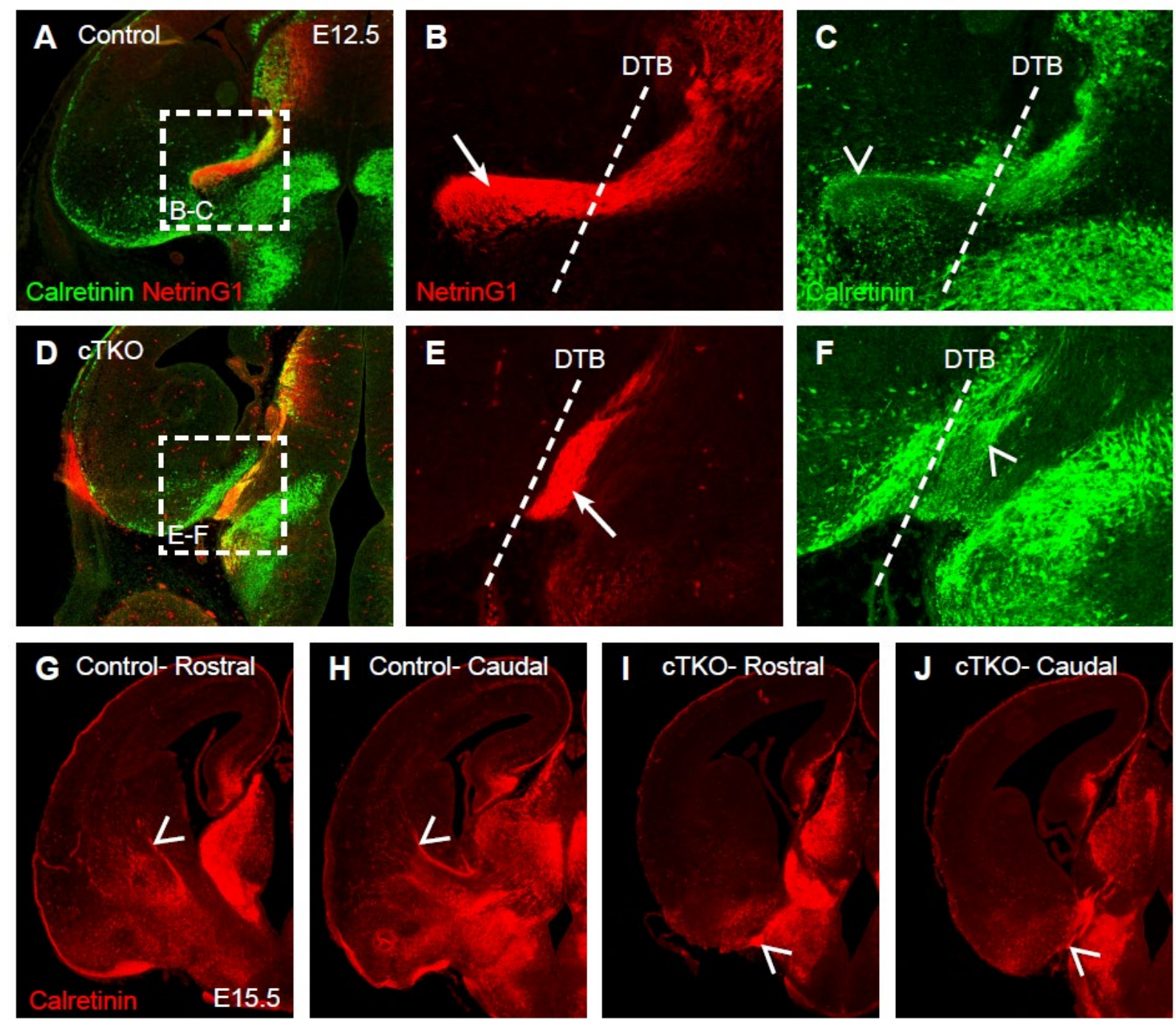

Figure A10. Calretinin-positive axons fail to cross into the telencephalon in cTKO

embryos. A-C. E13.5 embryonic mouse brains are labeled with NetrinG1, which labels most thalamocortical axons, and Calretinin, which labels axons from the lateral thalamus. Both

NetrinG1 (arrow, B) and Calretinin (open arrowhead, C) positive axons cross the diencephalontelencephalon boundary (DTB) in control brains. D-F. E13.5 cTKO brains are labeled with NetrinG1 (arrow, E) and Calretinin (open arrowhead, F), neither of which cross the DTB. G-H. Calretinin-positive thalamocortical axons (open arrowheads) are located in the telencephalon at E15.5 in control brains at both rostral and caudal levels. I-J. In E15.5 cTKO brains, Calretinin- 
positive axons are located at the DTB region in rostral regions (open arrowhead, I), and are coursing ventrally towards the hypothalamus at caudal levels (open arrowhead, J). 

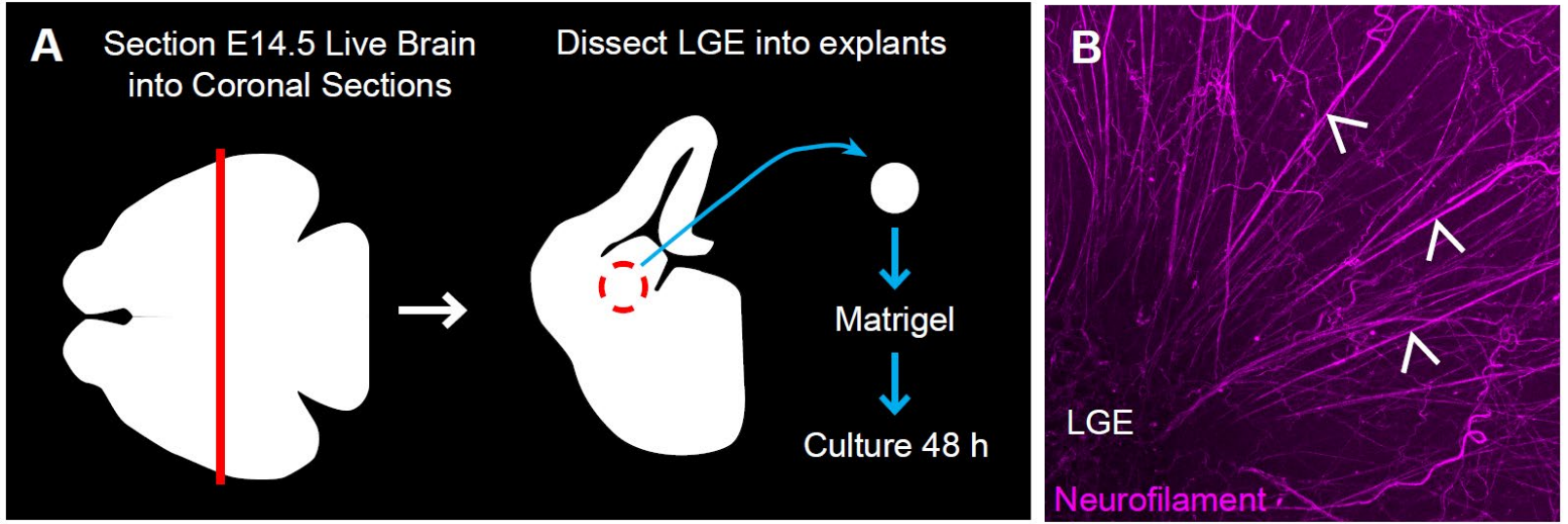

Figure A11. Explants from the developing striatum extend axons in Matrigel. A. E14.5 embryonic brains are sectioned coronally, the lateral ganglionic eminence (LGE) is dissected out from slices, and explants are taken to be grown in vitro in Matrigel for 48 hours. B. LGE explant (lower left) extends axons (open arrowheads) after being grown in Matrigel, which can be stained with the axonal marker Neurofilament. 

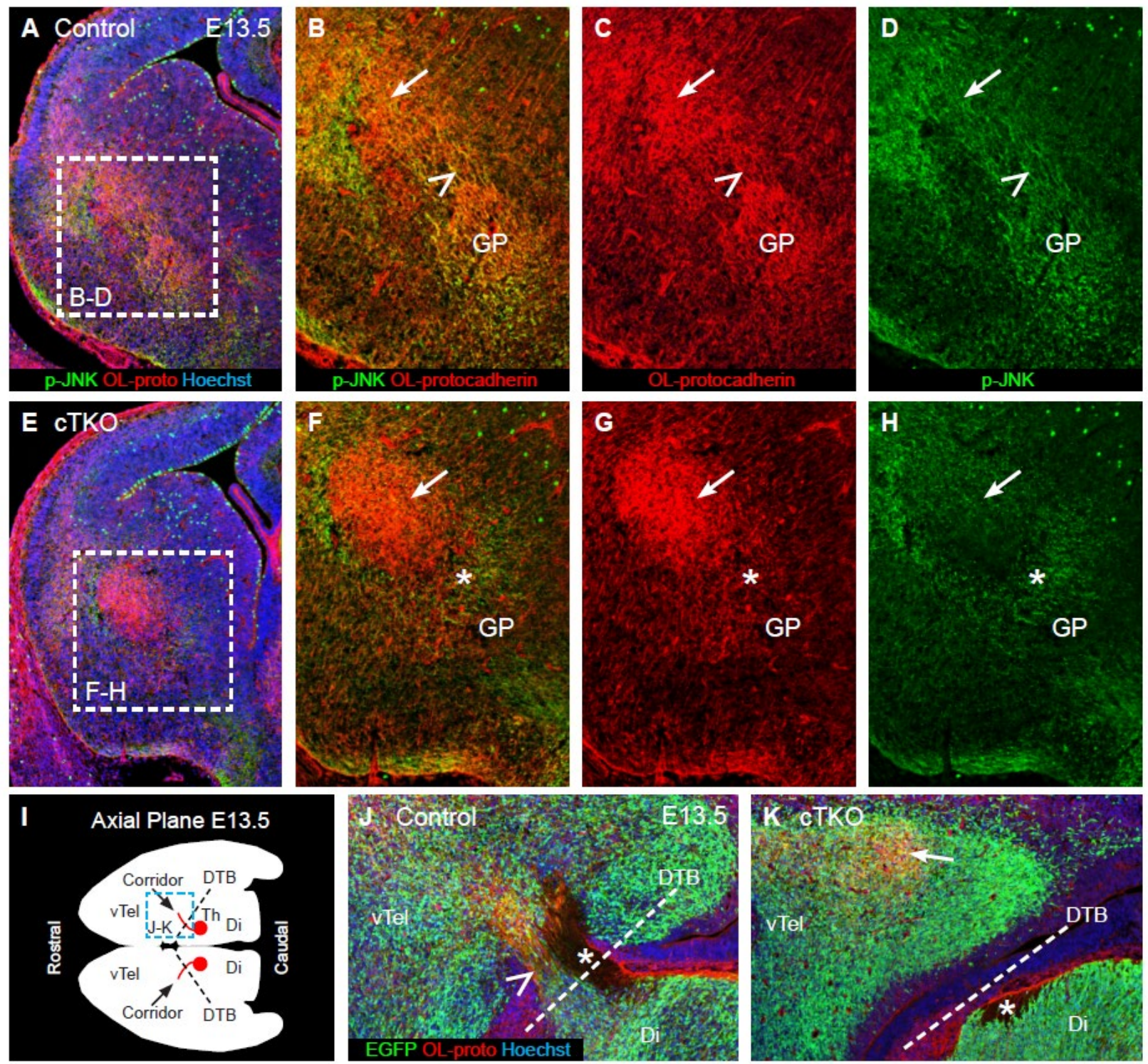

Figure A12. OL-protocadherin striatal axons fail to extend in the cTKO mouse. A-D. E13.5 control brains labeled with OL-protocadherin, a developing striatal marker, and p-JNK. The nascent striatum (arrow) is labeled with OL-protocadherin, which extends axons to the globus pallidus (GP, open arrowheads). These axons are p-JNK positive. E-H. In the cTKO brain, the OL-protocadherin positive striatum (arrow) fails to send axons (asterisk) to the GP, and is not pJNK positive. I-J. In the axial view, OL-protocadherin positive axons (open arrowhead) can be seen crossing the DTB in a control brain rostral to where thalamocortical axons course 
(asterisk). K. In cTKO brains, the OL-protocadherin positive domain is contained to the ventral telencephalon, and no axons are observed crossing the DTB (asterisk). 

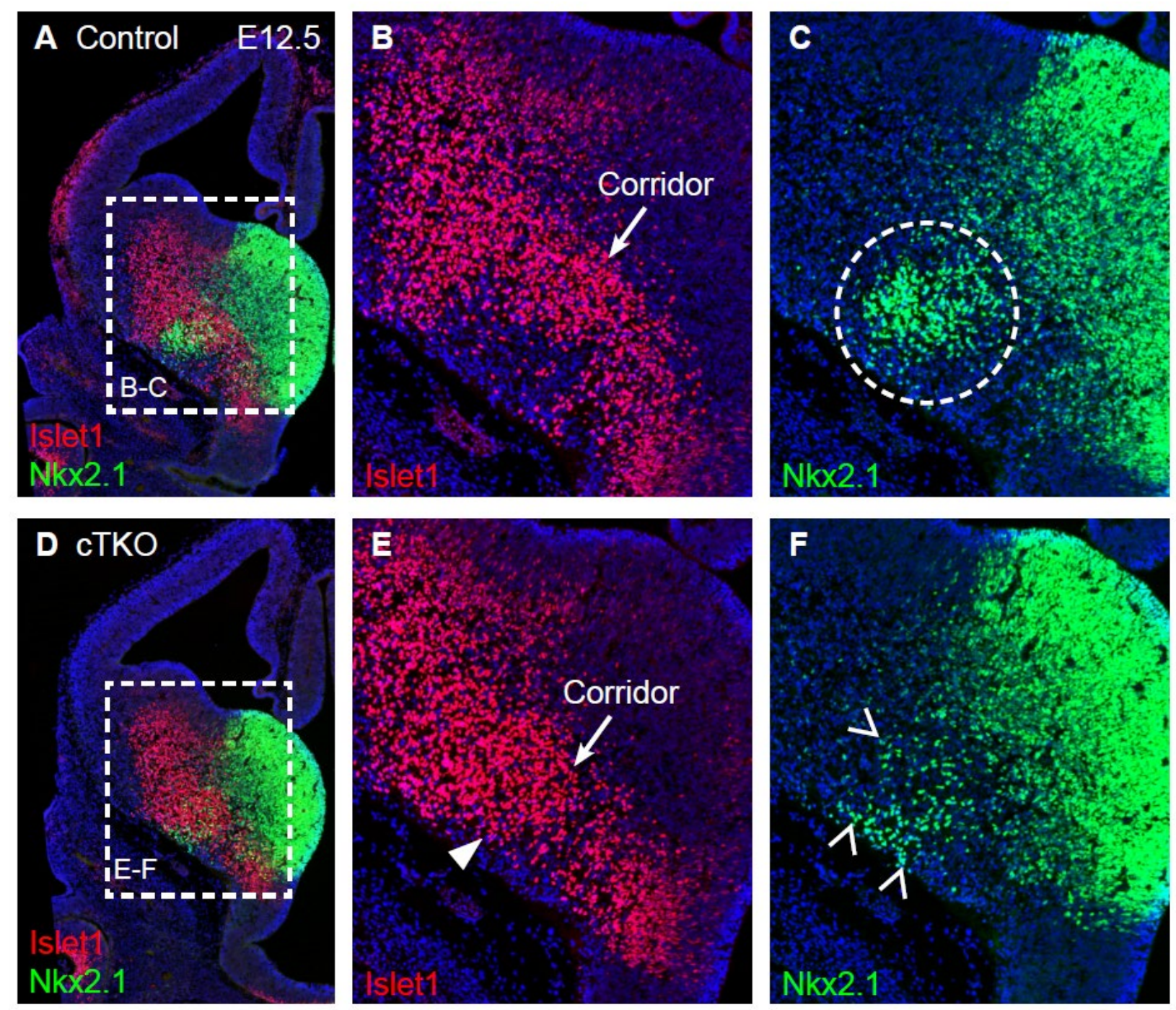

Figure A13. Corridor cells are present ectopically in the nascent globus pallidus in cTKO

mice at E12.5. A-C. E12.5 embryonic mouse brain stained for Islet1, a marker of corridor cells, and Nkx2.1, which labels the medial ganglionic eminence and globus pallidus. B. Islet1-positive corridor cells (arrow) are located between the two Nkx2.1-positive domains. C. The globus pallidus (encircled) is compact and located in the ventrolateral telencephalon. D-F. E12.5 cTKO embryonic mouse brain labeled with Islet1 and Nkx2.1. E. Islet1-postiive cells are located in both a location similar to controls (arrow), but also have extended ventrally to invade the globus pallidus region (closed arrowhead). F. The globus pallidus is spread out over a wider territory (open arrowheads), and appears is less cell dense. 

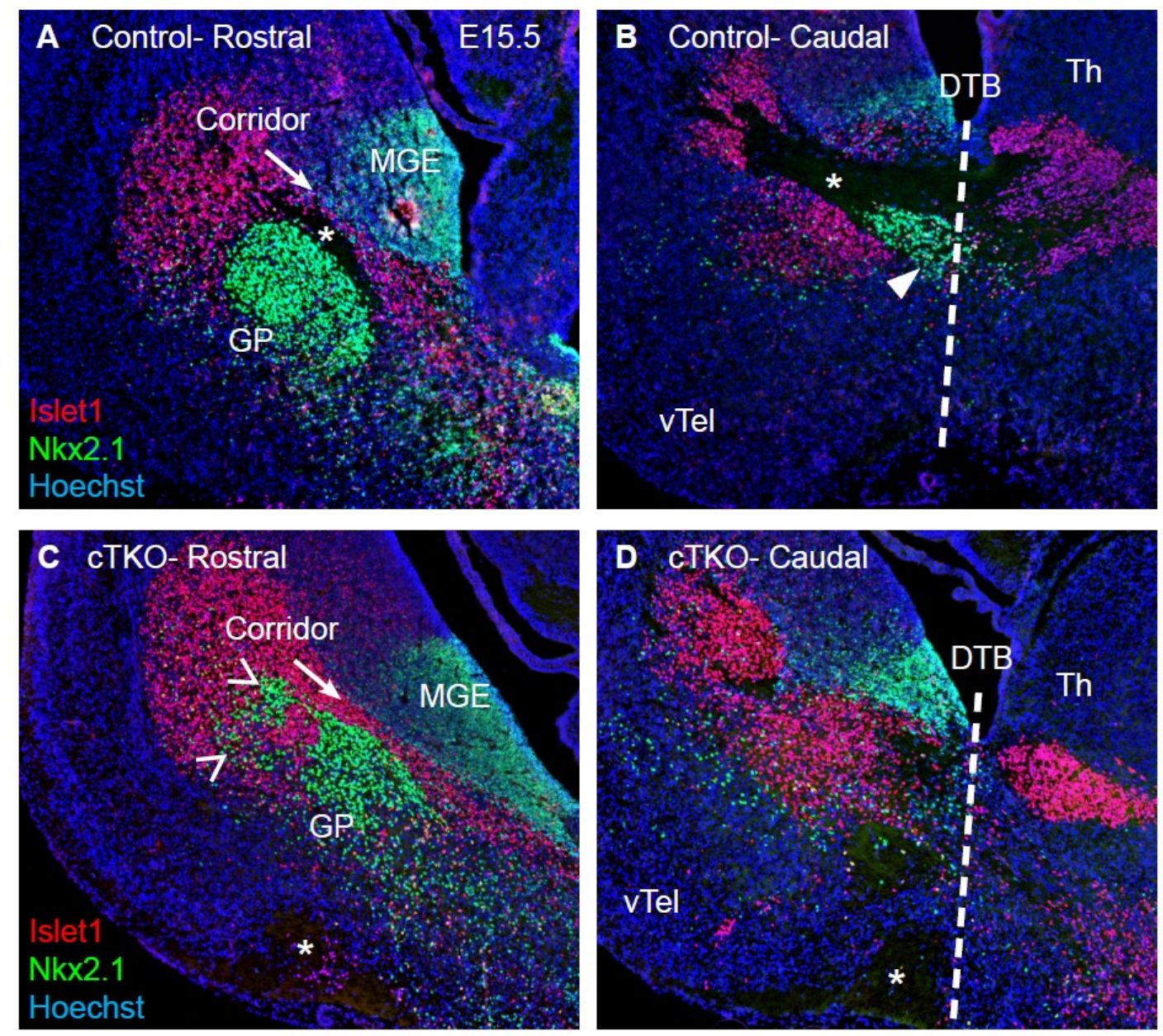

Figure A14. The developing striatum is morphologically altered in the cTKO ventral

telencephalon. A-B. Islet1 and Nkx2.1 labeling of E15.5 control embryos. A. Islet1-labeled corridor cells (arrow) extend through the Nkx2.1-positive medial ganglionic eminence (MGE) and globus pallidus (GP). B. The GP (closed arrowhead) resides at the diencephalontelencephalon boundary (DTB), located directly beneath thalamocortical axons (asterisk). C-D. Islet1- and Nkx2.1-labeled cTKO brains at E15.5. C. The Islet1-positive corridor (arrow) extends through the MGE and GP, however the GP is displaced laterally (open arrowheads) and splits into two separate structures. D. The Islet1 and Nkx2.1 expression domains are expanded compared to controls, and thalamocortical axons form bundles along the ventral telencephalon. 

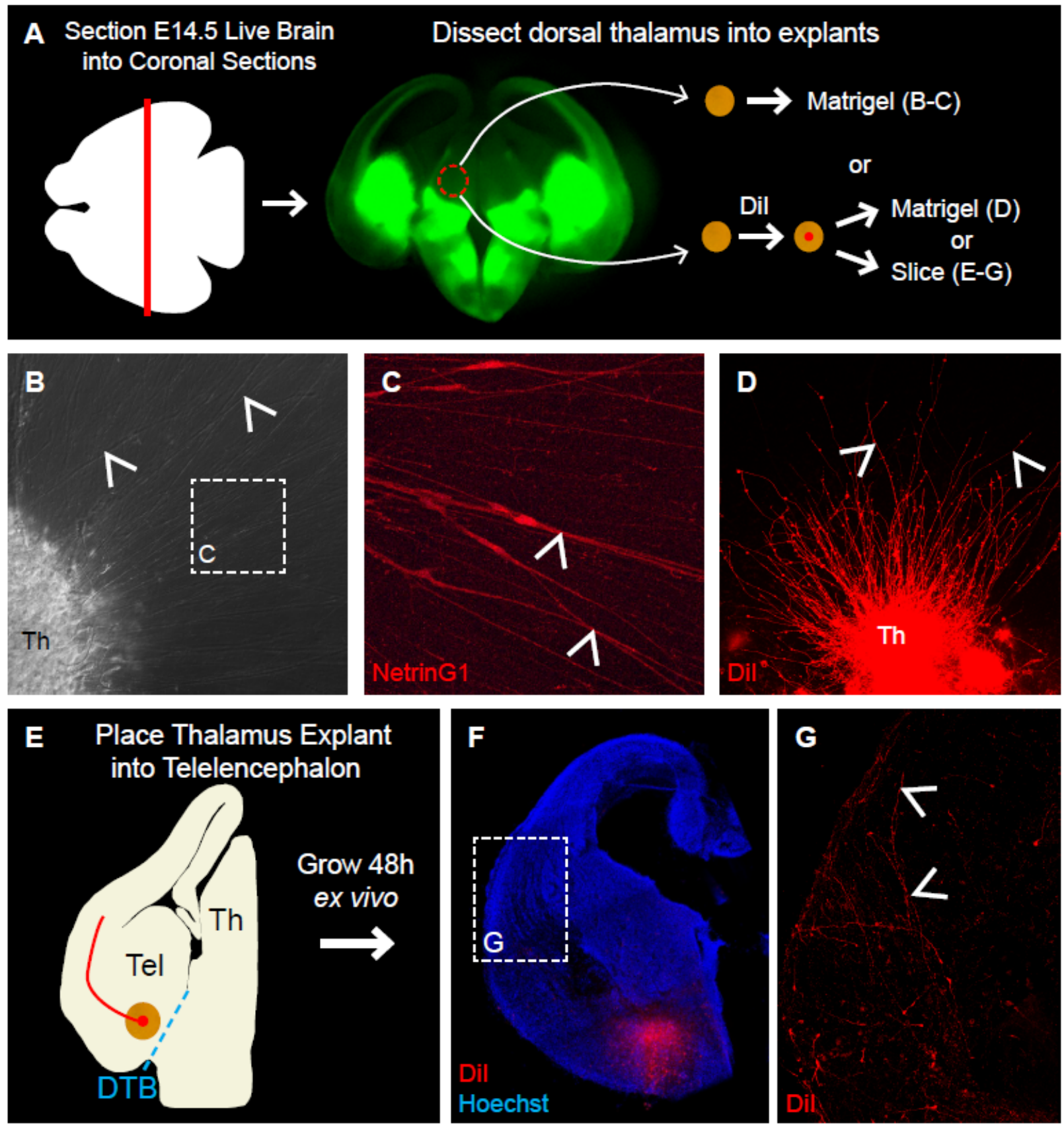

Figure A15. Transplanted thalamic explants extend axons in Matrigel and slice cultures.

A. E14.5 DIx5/6-CIE brains are sectioned in the coronal plane, the thalamus is removed from slices, and explants are generated to be placed either in Matrigel or in brain slices. B-C. Thalamus (Th) explants grown in Matrigel extend axons (open arrowheads, B) out from the explant (lower left), which can be labeled with the thalamocortical axon marker NetrinG1 (open 
arrowheads, C). D. Dil-labeling of axons (open arrowheads, D) grown from a thalamus explant that had Dil inserted before being placed in the Matrigel. E-G. A Dil-labeled thalamic explant is placed onto the telencephalic side of the diencephalon-telencephalon boundary (DTB) in a brain slice, and has extended axons towards the cortex after 48 hours of growth ex vivo (open arrowheads, G). 

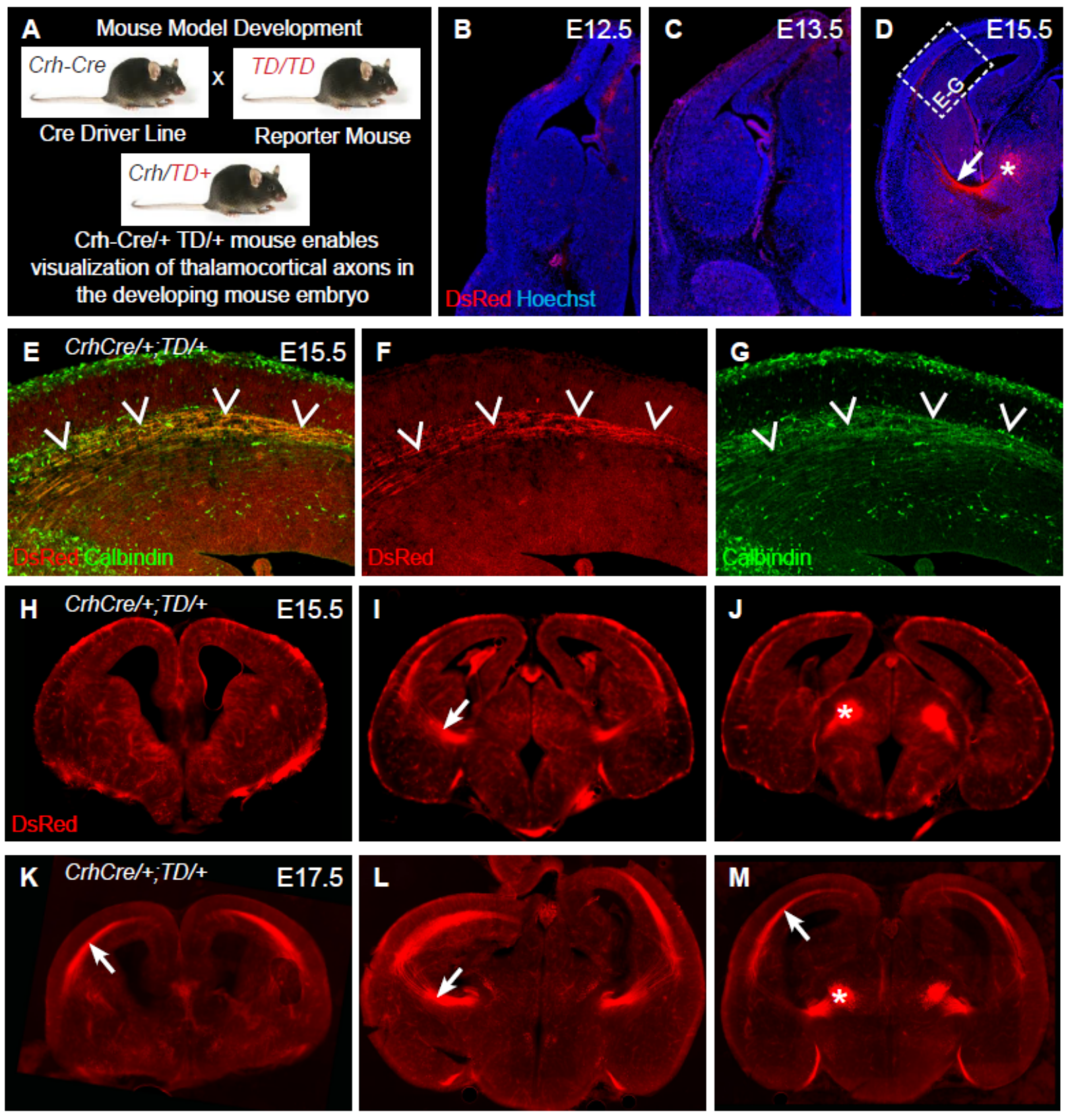

Figure A16. The CrhCre mouse line can be used to label thalamocortical axons at late,

but not early, embryonic time points. A. Genetic cross used to generate a mouse expressing both the Corticotropin-releasing hormone (Crh) driver line and the red fluorescent reporter tandem dimer (TD) Tomato, which enables visualization of thalamocortical axons in mice. B-D. Crh-Cre/+; TD/+ mice at embryonic (E) days $12.5(B), 13.5(\mathrm{C})$, and $15.5(\mathrm{D})$, reveal that expression of Crh-Cre begins after E13.5, and is evident by E15.5 in the mouse in both the 
thalamus (asterisk, D) and axons in the internal capsule (arrow, D) and cortex. E-G. In the CrhCre/+; TD/+ mouse cortex, axons (open arrowheads) are co-labeled for DsRed (a red fluorescent protein) and Calbindin, suggesting that Calbindin-positive axons are at least in part thalamocortical axons. H-J. E15.5 vibratome sections of a Crh-Cre/+; TD/+ brain shows labeling of axons in the thalamus (asterisk, J), internal capsule (arrow, I), and few axons in the cortex (I). K-M. E17.5 vibratome sections of a Crh-Cre/+; TD/+ brain labels the thalamus (asterisk, M) and a larger number of axons in the internal capsule and cortex (arrows) compared to the E15.5 embryo $(\mathrm{H}-\mathrm{I})$. 
References 
Abe, P., Molnar, Z., Tzeng, Y. S., Lai, D. M., Arnold, S. J. and Stumm, R. (2015). Intermediate Progenitors Facilitate Intracortical Progression of Thalamocortical Axons and Interneurons through CXCL12 Chemokine Signaling. J Neurosci 35, 13053-13063.

Abe, P., Mueller, W., Schütz, D., MacKay, F., Thelen, M., Zhang, P. and Stumm, R. (2014). CXCR7 prevents excessive CXCL12-mediated downregulation of CXCR4 in migrating cortical interneurons. Development 141, 1857-1863.

Adams, N. C., Lozsadi, D. A. and Guillery, R. W. (1997). Complexities in the thalamocortical and corticothalamic pathways. Eur J Neurosci 9, 204-209.

Aghakhani, Y., Bagshaw, A. P., Benar, C. G., Hawco, C., Andermann, F., Dubeau, F. and Gotman, J. (2004). fMRI activation during spike and wave discharges in idiopathic generalized epilepsy. Brain 127, 1127-1144.

Agmon, A., Yang, L. T., Jones, E. G. and O'Dowd, D. K. (1995). Topological precision in the thalamic projection to neonatal mouse barrel cortex. J Neurosci 15, 549-561.

Amir, R. E., Van den Veyver, I. B., Wan, M., Tran, C. Q., Francke, U. and Zoghbi, H. Y. (1999). Rett syndrome is caused by mutations in X-linked MECP2, encoding methyl-CpG-binding protein 2. Nat Genet 23, 185-188.

Anderson, S. A., Eisenstat, D. D., Shi, L. and Rubenstein, J. L. (1997). Interneuron migration from basal forebrain to neocortex: dependence on Dlx genes. Science 278, 474-476.

Anderson, S. A., Marin, O., Horn, C., Jennings, K. and Rubenstein, J. L. (2001). Distinct cortical migrations from the medial and lateral ganglionic eminences. Development 128, 353-363.

Ang, E. S., Jr., Haydar, T. F., Gluncic, V. and Rakic, P. (2003). Four-dimensional migratory coordinates of GABAergic interneurons in the developing mouse cortex. J Neurosci 23, 5805-5815.

Angevine, J. B., Jr. (1970). Time of neuron origin in the diencephalon of the mouse. An autoradiographic study. J Comp Neurol 139, 129-187.

Angevine, J. B., Jr. and Sidman, R. L. (1961). Autoradiographic study of cell migration during histogenesis of cerebral cortex in the mouse. Nature 192, 766-768.

Anticevic, A., Cole, M. W., Repovs, G., Murray, J. D., Brumbaugh, M. S., Winkler, A. M., Savic, A., Krystal, J. H., Pearlson, G. D. and Glahn, D. C. (2014). Characterizing thalamo-cortical disturbances in schizophrenia and bipolar illness. Cereb Cortex 24, 3116-3130.

Anton-Bolanos, N., Espinosa, A. and Lopez-Bendito, G. (2018). Developmental interactions between thalamus and cortex: a true love reciprocal story. Curr Opin Neurobiol 52, 33-41.

Arthur-Farraj, P. J., Latouche, M., Wilton, D. K., Quintes, S., Chabrol, E., Banerjee, A., Woodhoo, A., Jenkins, B., Rahman, M., Turmaine, M., et al. (2012). c-Jun reprograms Schwann cells of injured nerves to generate a repair cell essential for regeneration. Neuron 75, 633-647.

Asaoka, Y. and Nishina, H. (2010). Diverse physiological functions of MKK4 and MKK7 during early embryogenesis. J Biochem 148, 393-401.

Austin, C. P. and Cepko, C. L. (1990). Cellular migration patterns in the developing mouse cerebral cortex. Development 110, 713-732.

Bacci, A., Huguenard, J. R. and Prince, D. A. (2005). Modulation of Neocortical Interneurons: Extrinsic Influences and Exercises in Self-Control. Trends in neurosciences 28.

Backer, S., Lokmane, L., Landragin, C., Deck, M., Garel, S. and Bloch-Gallego, E. (2018). Trio GEF mediates RhoA activation downstream of Slit2 and coordinates telencephalic wiring. Development 145.

Bagri, A., Marin, O., Plump, A. S., Mak, J., Pleasure, S. J., Rubenstein, J. L. and Tessier-Lavigne, M. (2002). Slit proteins prevent midline crossing and determine the dorsoventral position of major axonal pathways in the mammalian forebrain. Neuron 33, 233-248. 
Barber, M., Andrews, W. D., Memi, F., Gardener, P., Ciantar, D., Tata, M., Ruhrberg, C. and Parnavelas, J. G. (2018). Vascular-Derived Vegfa Promotes Cortical Interneuron Migration and Proximity to the Vasculature in the Developing Forebrain. Cereb Cortex 28, 2577-2593.

Bartolini, G., Sanchez-Alcaniz, J. A., Osorio, C., Valiente, M., Garcia-Frigola, C. and Marin, O. (2017). Neuregulin 3 Mediates Cortical Plate Invasion and Laminar Allocation of GABAergic Interneurons. Cell Rep 18, 1157-1170.

Baudoin, J. P., Viou, L., Launay, P. S., Luccardini, C., Espeso Gil, S., Kiyasova, V., Irinopoulou, T., Alvarez, C., Rio, J. P., Boudier, T., et al. (2012). Tangentially migrating neurons assemble a primary cilium that promotes their reorientation to the cortical plate. Neuron 76, 1108-1122.

Beaulieu, C., Kisvarday, Z., Somogyi, P., Cynader, M. and Cowey, A. (1992). Quantitative distribution of GABA-immunopositive and -immunonegative neurons and synapses in the monkey striate cortex (area 17). Cereb Cortex 2, 295-309.

Bellion, A., Baudoin, J. P., Alvarez, C., Bornens, M. and Metin, C. (2005). Nucleokinesis in tangentially migrating neurons comprises two alternating phases: forward migration of the Golgi/centrosome associated with centrosome splitting and myosin contraction at the rear. $J$ Neurosci 25, 5691-5699.

Bennett, B. L., Sasaki, D. T., Murray, B. W., O'Leary, E. C., Sakata, S. T., Xu, W., Leisten, J. C., Motiwala, A., Pierce, S., Satoh, Y., et al. (2001). SP600125, an anthrapyrazolone inhibitor of Jun N-terminal kinase. Proc Natl Acad Sci U S A 98, 13681-13686.

Berry, M. and Rogers, A. W. (1965). The migration of neuroblasts in the developing cerebral cortex. J Anat 99, 691-709.

Bielle, F., Marcos-Mondejar, P., Keita, M., Mailhes, C., Verney, C., Nguyen Ba-Charvet, K., TessierLavigne, M., Lopez-Bendito, G. and Garel, S. (2011a). Slit2 activity in the migration of guidepost neurons shapes thalamic projections during development and evolution. Neuron 69, 1085-1098.

Bielle, F., Marcos-Mondejar, P., Leyva-Diaz, E., Lokmane, L., Mire, E., Mailhes, C., Keita, M., Garcia, N., Tessier-Lavigne, M., Garel, S., et al. (2011b). Emergent growth cone responses to combinations of Slit1 and Netrin 1 in thalamocortical axon topography. Curr Biol 21, 1748-1755.

Bjorkblom, B., Ostman, N., Hongisto, V., Komarovski, V., Filen, J. J., Nyman, T. A., Kallunki, T., Courtney, M. J. and Coffey, E. T. (2005). Constitutively active cytoplasmic c-Jun N-terminal kinase 1 is a dominant regulator of dendritic architecture: role of microtubule-associated protein 2 as an effector. J Neurosci 25, 6350-6361.

Bjorkblom, B., Vainio, J. C., Hongisto, V., Herdegen, T., Courtney, M. J. and Coffey, E. T. (2008). All JNKs can kill, but nuclear localization is critical for neuronal death. J Biol Chem 283, 19704-19713.

Bogoyevitch, M. A. and Kobe, B. (2006). Uses for JNK: the many and varied substrates of the C-Jun Nterminal kinases. Microbiol Mol Biol Rev 70, 1061-1095.

Bonneau, D., Toutain, A., Laquerriere, A., Marret, S., Saugier-Veber, P., Barthez, M. A., Radi, S., BiranMucignat, V., Rodriguez, D. and Gelot, A. (2002). X-linked lissencephaly with absent corpus callosum and ambiguous genitalia (XLAG): clinical, magnetic resonance imaging, and neuropathological findings. Ann Neurol 51, 340-349.

Bortone, D. and Polleux, F. (2009). KCC2 expression promotes the termination of cortical interneuron migration in a voltage-sensitive calcium-dependent manner. Neuron 62, 53-71.

Botella-Lopez, A., Garcia-Lopez, R., Pombero, A. and Martinez, S. (2019). Radial glia fibers translate Fgf8 morphogenetic signals to generate a thalamic nuclear complex protomap in the mantle layer. Brain Struct Funct 224, 661-679.

Braisted, J. E., Catalano, S. M., Stimac, R., Kennedy, T. E., Tessier-Lavigne, M., Shatz, C. J. and O'Leary, D. D. (2000). Netrin-1 promotes thalamic axon growth and is required for proper development of the thalamocortical projection. J Neurosci 20, 5792-5801. 
Braisted, J. E., Ringstedt, T. and O'Leary, D. D. (2009). Slits are chemorepellents endogenous to hypothalamus and steer thalamocortical axons into ventral telencephalon. Cereb Cortex 19 Suppl 1, i144-151.

Braisted, J. E., Tuttle, R. and O'Leary D, D. (1999). Thalamocortical axons are influenced by chemorepellent and chemoattractant activities localized to decision points along their path. Dev Biol 208, 430-440.

Brecht, S., Kirchhof, R., Chromik, A., Willesen, M., Nicolaus, T., Raivich, G., Wessig, J., Waetzig, V., Goetz, M., Claussen, M., et al. (2005). Specific pathophysiological functions of JNK isoforms in the brain. Eur J Neurosci 21, 363-377.

Broca, P. (1878). Anatomie comparee des circonvolution cerebrales: le grande lobe limbique et la scissure limbiquedans la s erie des mammiferes. Rev D'Anthropo 1:385-49.

Brodmann (1909). Vergleichende Lokalisationslehre der Grosshirnrinde in ihren Prinzipien dargestellt auf Grund des Zeelenbaues. Barth, Leipzig, Germany.

Brose, K., Bland, K. S., Wang, K. H., Arnott, D., Henzel, W., Goodman, C. S., Tessier-Lavigne, M. and Kidd, T. (1999). Slit proteins bind Robo receptors and have an evolutionarily conserved role in repulsive axon guidance. Cell 96, 795-806.

Brown, K. N., Chen, S., Han, Z., Lu, C. H., Tan, X., Zhang, X. J., Ding, L., Lopez-Cruz, A., Saur, D., Anderson, S. A., et al. (2011). Clonal production and organization of inhibitory interneurons in the neocortex. Science 334, 480-486.

Butt, S. J., Fuccillo, M., Nery, S., Noctor, S., Kriegstein, A., Corbin, J. G. and Fishell, G. (2005). The temporal and spatial origins of cortical interneurons predict their physiological subtype. Neuron 48, 591-604.

Campbell, K. and Gotz, M. (2002). Radial glia: multi-purpose cells for vertebrate brain development. Trends Neurosci 25, 235-238.

Canu, E., Agosta, F. and Filippi, M. (2015). A selective review of structural connectivity abnormalities of schizophrenic patients at different stages of the disease. Schizophr Res 161, 19-28.

Carrie, A., Jun, L., Bienvenu, T., Vinet, M. C., McDonell, N., Couvert, P., Zemni, R., Cardona, A., Van Buggenhout, G., Frints, S., et al. (1999). A new member of the IL-1 receptor family highly expressed in hippocampus and involved in X-linked mental retardation. Nat Genet 23, 25-31.

Casanova, M. F., El-Baz, A. S., Kamat, S. S., Dombroski, B. A., Khalifa, F., Elnakib, A., Soliman, A., Allison-McNutt, A. and Switala, A. E. (2013). Focal cortical dysplasias in autism spectrum disorders. Acta Neuropathol Commun 1, 67.

Catalano, S. M., Robertson, R. T. and Killackey, H. P. (1996). Individual axon morphology and thalamocortical topography in developing rat somatosensory cortex. J Comp Neurol 367, 36-53.

Cauli, B., Audinat, E., Lambolez, B., Angulo, M. C., Ropert, N., Tsuzuki, K., Hestrin, S. and Rossier, J. (1997). Molecular and physiological diversity of cortical nonpyramidal cells. J Neurosci 17, 38943906.

Caviness, V. S., Jr. and Frost, D. O. (1980). Tangential organization of thalamic projections to the neocortex in the mouse. J Comp Neurol 194, 335-367.

Chahrour, M. and Zoghbi, H. Y. (2007). The story of Rett syndrome: from clinic to neurobiology. Neuron 56, 422-437.

Chang, L., Jones, Y., Ellisman, M. H., Goldstein, L. S. and Karin, M. (2003). JNK1 is required for maintenance of neuronal microtubules and controls phosphorylation of microtubule-associated proteins. Dev Cell 4, 521-533.

Chao, H. T., Chen, H., Samaco, R. C., Xue, M., Chahrour, M., Yoo, J., Neul, J. L., Gong, S., Lu, H. C., Heintz, N., et al. (2010). Dysfunction in GABA signalling mediates autism-like stereotypies and Rett syndrome phenotypes. Nature 468, 263-269. 
Chatterjee, M., Li, K., Chen, L., Maisano, X., Guo, Q., Gan, L. and Li, J. Y. (2012). Gbx2 regulates thalamocortical axon guidance by modifying the LIM and Robo codes. Development 139, 46334643.

Chen, J. T., Lu, D. H., Chia, C. P., Ruan, D. Y., Sabapathy, K. and Xiao, Z. C. (2005). Impaired long-term potentiation in c-Jun N-terminal kinase 2-deficient mice. J Neurochem 93, 463-473.

Cho, I. H., Lee, K. W., Ha, H. Y. and Han, P. L. (2011). JNK/stress-activated protein kinase associated protein 1 is required for early development of telencephalic commissures in embryonic brains. Exp Mol Med 43, 462-470.

Chotard, C. and Salecker, I. (2004). Neurons and Glia: Team Players in Axon Guidance. Trends in neurosciences 27, 655-661.

Ciceri, G., Dehorter, N., Sols, I., Huang, Z. J., Maravall, M. and Marin, O. (2013). Lineage-specific laminar organization of cortical GABAergic interneurons. Nat Neurosci 16, 1199-1210.

Coffey, E. T. (2014). Nuclear and cytosolic JNK signalling in neurons. Nat Rev Neurosci 15, 285-299.

Coffey, E. T., Hongisto, V., Dickens, M., Davis, R. J. and Courtney, M. J. (2000). Dual Roles for c-Jun NTerminal Kinase in Developmental and Stress Responses in Cerebellar Granule Neurons. In J Neurosci, pp. 7602-7613.

Coffey, E. T., Smiciene, G., Hongisto, V., Cao, J., Brecht, S., Herdegen, T. and Courtney, M. J. (2002). cJun N-Terminal Protein Kinase (JNK) 2/3 Is Specifically Activated by Stress, Mediating c-Jun Activation, in the Presence of Constitutive JNK1 Activity in Cerebellar Neurons. In J Neurosci, pp. 4335-4345.

Coso, O. A., Chiariello, M., Yu, J. C., Teramoto, H., Crespo, P., Xu, N., Miki, T. and Gutkind, J. S. (1995). The small GTP-binding proteins Rac1 and Cdc42 regulate the activity of the JNK/SAPK signaling pathway. Cell 81, 1137-1146.

Cristofori, I., Cohen-Zimerman, S. and Grafman, J. (2019). Executive functions. Handb Clin Neurol 163, 197-219.

Cummings, D., Malun, D. and Brunjes, P. (1997). Development of the Anterior Commissure in the Opossum: Midline Extracellular Space and Glia Coincide With Early Axon Decussation. Journal of neurobiology 32.

Cuzon, V. C., Yeh, P. W., Cheng, Q. and Yeh, H. H. (2006). Ambient GABA promotes cortical entry of tangentially migrating cells derived from the medial ganglionic eminence. Cereb Cortex 16, 13771388.

Dani, V. S., Chang, Q., Maffei, A., Turrigiano, G. G., Jaenisch, R. and Nelson, S. B. (2005). Reduced cortical activity due to a shift in the balance between excitation and inhibition in a mouse model of Rett Syndrome. In Proc Natl Acad Sci U S A, pp. 12560-12565.

Das, M., Jiang, F., Sluss, H. K., Zhang, C., Shokat, K. M., Flavell, R. A. and Davis, R. J. (2007). Suppression of p53-dependent senescence by the JNK signal transduction pathway. Proc Natl Acad Sci U S A 104, 15759-15764.

Davis, R. J. (2000). Signal transduction by the JNK group of MAP kinases. Cell 103, 239-252.

Dawson, D. R. and Killackey, H. P. (1985). Distinguishing topography and somatotopy in the thalamocortical projections of the developing rat. Brain Res 349, 309-313.

de Anda, F. C., Rosario, A. L., Durak, O., Tran, T., Graff, J., Meletis, K., Rei, D., Soda, T., Madabhushi, R., Ginty, D. D., et al. (2012). Autism spectrum disorder susceptibility gene TAOK2 affects basal dendrite formation in the neocortex. Nat Neurosci 15, 1022-1031.

de Carlos, J. A., Lopez-Mascaraque, L. and Valverde, F. (1996). Dynamics of cell migration from the lateral ganglionic eminence in the rat. J Neurosci 16, 6146-6156.

Deacon, T. W., Pakzaban, P. and Isacson, O. (1994). The lateral ganglionic eminence is the origin of cells committed to striatal phenotypes: neural transplantation and developmental evidence. Brain Res 668, 211-219. 
Decaillot, F. M., Kazmi, M. A., Lin, Y., Ray-Saha, S., Sakmar, T. P. and Sachdev, P. (2011). CXCR7/CXCR4 heterodimer constitutively recruits beta-arrestin to enhance cell migration. J Biol Chem 286, 32188-32197.

DeGeer, J., Kaplan, A., Mattar, P., Morabito, M., Stochaj, U., Kennedy, T. E., Debant, A., Cayouette, M., Fournier, A. E. and Lamarche-Vane, N. (2015). Hsc70 chaperone activity underlies Trio GEF function in axon growth and guidance induced by netrin-1. J Cell Biol 210, 817-832.

Denaxa, M., Chan, C. H., Schachner, M., Parnavelas, J. G. and Karagogeos, D. (2001). The adhesion molecule TAG-1 mediates the migration of cortical interneurons from the ganglionic eminence along the corticofugal fiber system. Development 128, 4635-4644.

Derijard, B., Hibi, M., Wu, I. H., Barrett, T., Su, B., Deng, T., Karin, M. and Davis, R. J. (1994). JNK1: a protein kinase stimulated by UV light and Ha-Ras that binds and phosphorylates the c-Jun activation domain. Cell 76, 1025-1037.

Di Cristo, G. (2007). Development of cortical GABAergic circuits and its implications for neurodevelopmental disorders. Clin Genet 72, 1-8.

Doherty, J. L. and Owen, M. J. (2014). Genomic insights into the overlap between psychiatric disorders: implications for research and clinical practice. Genome Med 6, 29.

Dubos, A., Meziane, H., lacono, G., Curie, A., Riet, F., Martin, C., Loaëc, N., Birling, M. C., Selloum, M., Normand, E., et al. (2018). A new mouse model of ARX dup24 recapitulates the patients' behavioral and fine motor alterations. Hum Mol Genet 27, 2138-2153.

Dufour, A., Seibt, J., Passante, L., Depaepe, V., Ciossek, T., Frisen, J., Kullander, K., Flanagan, J. G., Polleux, F. and Vanderhaeghen, P. (2003). Area specificity and topography of thalamocortical projections are controlled by ephrin/Eph genes. Neuron 39, 453-465.

Dupin, I., Lokmane, L., Dahan, M., Garel, S. and Studer, V. (2015). Subrepellent doses of Slit1 promote Netrin-1 chemotactic responses in subsets of axons. Neural Dev 10, 5.

Elias, L. A., Turmaine, M., Parnavelas, J. G. and Kriegstein, A. R. (2010). Connexin 43 mediates the tangential to radial migratory switch in ventrally derived cortical interneurons. J Neurosci 30 7072-7077.

Fagiolini, M. and Hensch, T. (2000). Inhibitory Threshold for Critical-Period Activation in Primary Visual Cortex. Nature 404.

Fatemi, S. H., Halt, A. R., Stary, J. M., Kanodia, R., Schulz, S. C. and Realmuto, G. R. (2002). Glutamic acid decarboxylase 65 and $67 \mathrm{kDa}$ proteins are reduced in autistic parietal and cerebellar cortices. Biol Psychiatry 52, 805-810.

Fazel Darbandi, S., Poitras, L., Monis, S., Lindtner, S., Yu, M., Hatch, G., Rubenstein, J. L. and Ekker, M. (2016). Functional consequences of I56ii Dlx enhancer deletion in the developing mouse forebrain. Dev Biol.

Feng, J., Xian, Q., Guan, T., Hu, J., Wang, M., Huang, Y., So, K. F., Evans, S. M., Chai, G., Goffinet, A. M., et al. (2016). Celsr3 and Fzd3 Organize a Pioneer Neuron Scaffold to Steer Growing Thalamocortical Axons. Cereb Cortex 26, 3323-3334.

Fenstermaker, A. G., Prasad, A. A., Bechara, A., Adolfs, Y., Tissir, F., Goffinet, A., Zou, Y. and Pasterkamp, R. J. (2010). Wnt/planar cell polarity signaling controls the anterior-posterior organization of monoaminergic axons in the brainstem. J Neurosci 30, 16053-16064.

Fentress, J. C., Stanfield, B. B. and Cowan, W. M. (1981). Observation on the development of the striatum in mice and rats. Anat Embryol (Berl) 163, 275-298.

Ferrer, I., Blanco, R., Carmona, M. and Puig, B. (2001). Phosphorylated mitogen-activated protein kinase (MAPK/ERK-P), protein kinase of $38 \mathrm{kDa}$ (p38-P), stress-activated protein kinase (SAPK/JNK-P), and calcium/calmodulin-dependent kinase II (CaM kinase II) are differentially expressed in tau deposits in neurons and glial cells in tauopathies. J Neural Transm (Vienna) 108, 1397-1415. 
Ferrer, I., Pastor, P., Rey, M. J., Munoz, E., Puig, B., Pastor, E., Oliva, R. and Tolosa, E. (2003). Tau phosphorylation and kinase activation in familial tauopathy linked to deln296 mutation. Neuropathol Appl Neurobiol 29, 23-34.

Flames, N., Long, J. E., Garratt, A. N., Fischer, T. M., Gassmann, M., Birchmeier, C., Lai, C., Rubenstein, J. L. and Marin, O. (2004). Short- and long-range attraction of cortical GABAergic interneurons by neuregulin-1. Neuron 44, 251-261.

Friocourt, G., Liu, J. S., Antypa, M., Rakic, S., Walsh, C. A. and Parnavelas, J. G. (2007). Both doublecortin and doublecortin-like kinase play a role in cortical interneuron migration. $J$ Neurosci 27, 3875-3883.

Fung, S. J., Fillman, S. G., Webster, M. J. and Shannon Weickert, C. (2014). Schizophrenia and bipolar disorder show both common and distinct changes in cortical interneuron markers. Schizophr Res 155, 26-30.

Fung, S. J., Webster, M. J., Sivagnanasundaram, S., Duncan, C., Elashoff, M. and Weickert, C. S. (2010). Expression of interneuron markers in the dorsolateral prefrontal cortex of the developing human and in schizophrenia. Am J Psychiatry 167, 1479-1488.

Garcia-Pena, C. M., Kim, M., Frade-Perez, D., Avila-Gonzalez, D., Tellez, E., Mastick, G. S., Tamariz, E. and Varela-Echavarria, A. (2014). Ascending midbrain dopaminergic axons require descending GAD65 axon fascicles for normal pathfinding. Front Neuroanat 8, 43.

García, M. T. and Harwell, C. (2017). Radial Glia in the Ventral Telencephalon. FEBS Lett 591, 3942-3959.

Gdalyahu, A., Ghosh, I., Levy, T., Sapir, T., Sapoznik, S., Fishler, Y., Azoulai, D. and Reiner, O. (2004). DCX, a new mediator of the JNK pathway. EMBO J 23, 823-832.

Gelman, D. M., Martini, F. J., Nobrega-Pereira, S., Pierani, A., Kessaris, N. and Marin, O. (2009). The embryonic preoptic area is a novel source of cortical GABAergic interneurons. J Neurosci 29, 9380-9389.

Gezelius, H. and Lopez-Bendito, G. (2017). Thalamic neuronal specification and early circuit formation. Dev Neurobiol 77, 830-843.

Godement, P., Vanselow, J., Thanos, S. and Bonhoeffer, F. (1987). A study in developing visual systems with a new method of staining neurones and their processes in fixed tissue. Development 101, 697-713.

Godin, J. D., Thomas, N., Laguesse, S., Malinouskaya, L., Close, P., Malaise, O., Purnelle, A., Raineteau, O., Campbell, K., Fero, M., et al. (2012). p27(Kip1) is a microtubule-associated protein that promotes microtubule polymerization during neuron migration. Dev Cell 23, 729-744.

Goodrich, L. V. and Strutt, D. (2011). Principles of planar polarity in animal development. Development 138, 1877-1892.

Gotman, J., Grova, C., Bagshaw, A., Kobayashi, E., Aghakhani, Y. and Dubeau, F. (2005). Generalized epileptic discharges show thalamocortical activation and suspension of the default state of the brain. Proc Natl Acad Sci U S A 102, 15236-15240.

Graczyk, P. P. (2013). JNK inhibitors as anti-inflammatory and neuroprotective agents. Future Med Chem 5, 539-551.

Guo, J., Otis, J. M., Higginbotham, H., Monckton, C., Cheng, J., Asokan, A., Mykytyn, K., Caspary, T., Stuber, G. D. and Anton, E. S. (2017). Primary Cilia Signaling Shapes the Development of Interneuronal Connectivity. Dev Cell 42, 286-300.e284.

Gupta, S., Barrett, T., Whitmarsh, A. J., Cavanagh, J., Sluss, H. K., Derijard, B. and Davis, R. J. (1996). Selective interaction of JNK protein kinase isoforms with transcription factors. Embo $j$ 15, 27602770.

Guye, M., Regis, J., Tamura, M., Wendling, F., McGonigal, A., Chauvel, P. and Bartolomei, F. (2006). The role of corticothalamic coupling in human temporal lobe epilepsy. Brain 129, 1917-1928. 
Ha, H. Y., Cho, I. H., Lee, K. W., Song, J. Y., Kim, K. S., Yu, Y. M., Lee, J. K., Song, J. S., Yang, S. D., Shin, H. S., et al. (2005). The axon guidance defect of the telencephalic commissures of the JSAP1deficient brain was partially rescued by the transgenic expression of JIP1. Dev Biol 277, 184-199.

Haider, B., Hausser, M. and Carandini, M. (2013). Inhibition dominates sensory responses in the awake cortex. Nature 493, 97-100.

Hand, R., Bortone, D., Mattar, P., Nguyen, L., Heng, J. I., Guerrier, S., Boutt, E., Peters, E., Barnes, A. P., Parras, C., et al. (2005). Phosphorylation of Neurogenin2 specifies the migration properties and the dendritic morphology of pyramidal neurons in the neocortex. Neuron 48, 45-62.

Hatanaka, Y., Zhu, Y., Torigoe, M., Kita, Y. and Murakami, F. (2016). From migration to settlement: the pathways, migration modes and dynamics of neurons in the developing brain. In Proc Jpn Acad Ser B Phys Biol Sci, pp. 1-19.

Haubst, N., Georges-Labouesse, E., De Arcangelis, A., Mayer, U. and Gotz, M. (2006). Basement membrane attachment is dispensable for radial glial cell fate and for proliferation, but affects positioning of neuronal subtypes. Development 133, 3245-3254.

Hendry, S. H., Schwark, H. D., Jones, E. G. and Yan, J. (1987). Numbers and proportions of GABAimmunoreactive neurons in different areas of monkey cerebral cortex. J Neurosci 7, 1503-1519.

Hensch, T. K. (2005). Critical period plasticity in local cortical circuits. Nat Rev Neurosci 6, 877-888.

Hestrin, S. and Galarreta, M. (2005). Electrical synapses define networks of neocortical GABAergic neurons. Trends Neurosci 28, 304-309.

Hevner, R. F., Daza, R. A., Englund, C., Kohtz, J. and Fink, A. (2004). Postnatal shifts of interneuron position in the neocortex of normal and reeler mice: evidence for inward radial migration. Neuroscience 124, 605-618.

Higginbotham, H., Eom, T. Y., Mariani, L. E., Bachleda, A., Hirt, J., Gukassyan, V., Cusack, C. L., Lai, C., Caspary, T. and Anton, E. S. (2012). Arl13b in primary cilia regulates the migration and placement of interneurons in the developing cerebral cortex. Dev Cell 23, 925-938.

Hirai, S., Cui, D. F., Miyata, T., Ogawa, M., Kiyonari, H., Suda, Y., Aizawa, S., Banba, Y. and Ohno, S. (2006). The c-Jun $\mathrm{N}$-terminal kinase activator dual leucine zipper kinase regulates axon growth and neuronal migration in the developing cerebral cortex. J Neurosci 26, 11992-12002.

Hirai, S., Izawa, M., Osada, S., Spyrou, G. and Ohno, S. (1996). Activation of the JNK pathway by distantly related protein kinases, MEKK and MUK. Oncogene 12, 641-650.

Hirai, S., Kawaguchi, A., Hirasawa, R., Baba, M., Ohnishi, T. and Ohno, S. (2002). MAPK-upstream protein kinase (MUK) regulates the radial migration of immature neurons in telencephalon of mouse embryo. Development 129, 4483-4495.

Hirano, S., Yan, Q. and Suzuki, S. T. (1999). Expression of a novel protocadherin, OL-protocadherin, in a subset of functional systems of the developing mouse brain. J Neurosci 19, 995-1005.

Horike, S., Cai, S., Miyano, M., Cheng, J. F. and Kohwi-Shigematsu, T. (2005). Loss of silent-chromatin looping and impaired imprinting of DLX5 in Rett syndrome. Nat Genet 37, 31-40.

Hsu, C. W., Wong, L., Rasmussen, T. L., Kalaga, S., McElwee, M. L., Keith, L. C., Bohat, R., Seavitt, J. R., Beaudet, A. L. and Dickinson, M. E. (2016). Three-dimensional microCT imaging of mouse development from early post-implantation to early postnatal stages. Dev Biol 419, 229-236.

Hu, H., Gan, J. and Jonas, P. (2014). Interneurons. Fast-spiking, parvalbumin(+) GABAergic interneurons: from cellular design to microcircuit function. Science 345, 1255263.

Hua, Z. L., Emiliani, F. E. and Nathans, J. (2015). Rac1 plays an essential role in axon growth and guidance and in neuronal survival in the central and peripheral nervous systems. Neural Dev 10, 21.

Hua, Z. L., Jeon, S., Caterina, M. J. and Nathans, J. (2014). Frizzled3 is required for the development of multiple axon tracts in the mouse central nervous system. Proc Natl Acad Sci U S A 111, E30053014. 
Huang, C., Rajfur, Z., Borchers, C., Schaller, M. D. and Jacobson, K. (2003). JNK phosphorylates paxillin and regulates cell migration. Nature 424, 219-223.

lida, C., Ohsawa, S., Taniguchi, K., Yamamoto, M., Morata, G. and Igaki, T. (2019). JNK-mediated SlitRobo signaling facilitates epithelial wound repair by extruding dying cells. Sci Rep 9, 19549.

Inada, H., Watanabe, M., Uchida, T., Ishibashi, H., Wake, H., Nemoto, T., Yanagawa, Y., Fukuda, A. and Nabekura, J. (2011). GABA regulates the multidirectional tangential migration of GABAergic interneurons in living neonatal mice. PLoS One 6, e27048.

Inan, M., Welagen, J. and Anderson, S. A. (2012). Spatial and temporal bias in the mitotic origins of somatostatin- and parvalbumin-expressing interneuron subgroups and the chandelier subtype in the medial ganglionic eminence. Cereb Cortex 22, 820-827.

Ito, M., Yoshioka, K., Akechi, M., Yamashita, S., Takamatsu, N., Sugiyama, K., Hibi, M., Nakabeppu, Y., Shiba, T. and Yamamoto, K. I. (1999). JSAP1, a Novel Jun N-Terminal Protein Kinase (JNK)Binding Protein That Functions as a Scaffold Factor in the JNK Signaling Pathway. In Mol Cell Biol, pp. 7539-7548.

Iwai, Y., Fagiolini, M., Obata, K. and Hensch, T. K. (2003). Rapid Critical Period Induction by Tonic Inhibition in Visual Cortex. The Journal of neuroscience : the official journal of the Society for Neuroscience 23.

Jaeschke, A., Karasarides, M., Ventura, J. J., Ehrhardt, A., Zhang, C., Flavell, R. A., Shokat, K. M. and Davis, R. J. (2006). JNK2 is a positive regulator of the cJun transcription factor. Mol Cell 23, 899911.

Jaiswal, M. K., Keros, S., Zhao, M., Inan, M., Schwartz, T. H., Anderson, S. A., Homanics, G. E. and Goldstein, P. A. (2015). Reduction in focal ictal activity following transplantation of MGE interneurons requires expression of the GABAA receptor alpha4 subunit. Front Cell Neurosci 9, 127.

Jessen, K. R. (2004). Glial Cells. The international journal of biochemistry \& cell biology 36.

Jia, Z., Guo, Y., Tang, Y., Xu, Q., Li, B. and Wu, Q. (2014). Regulation of the protocadherin Celsr3 gene and its role in globus pallidus development and connectivity. Mol Cell Biol 34, 3895-3910.

Jin, J., Suzuki, H., Hirai, S., Mikoshiba, K. and Ohshima, T. (2010). JNK phosphorylates Ser332 of doublecortin and regulates its function in neurite extension and neuronal migration. Dev Neurobiol 70, 929-942.

Jones, E. G. (1997). Cortical development and thalamic pathology in schizophrenia. Schizophr Bull 23, 483-501.

---- (2001). The thalamic matrix and thalamocortical synchrony. Trends Neurosci 24, 595-601.

---- (2007). The Thalamus. Cambridge University Press.

Jones, E. G. and Rakic, P. (2010). Radial columns in cortical architecture: it is the composition that counts. Cereb Cortex 20, 2261-2264.

Jones, L., Lopez-Bendito, G., Gruss, P., Stoykova, A. and Molnar, Z. (2002). Pax6 is required for the normal development of the forebrain axonal connections. Development 129, 5041-5052.

Kappeler, C., Saillour, Y., Baudoin, J. P., Tuy, F. P., Alvarez, C., Houbron, C., Gaspar, P., Hamard, G., Chelly, J., Metin, C., et al. (2006). Branching and nucleokinesis defects in migrating interneurons derived from doublecortin knockout mice. Hum Mol Genet 15, 1387-1400.

Kariminejad, R., Lind-Thomsen, A., Tumer, Z., Erdogan, F., Ropers, H. H., Tommerup, N., Ullmann, R. and Moller, R. S. (2011). High frequency of rare copy number variants affecting functionally related genes in patients with structural brain malformations. Hum Mutat 32, 1427-1435.

Karlsgodt, K. H., Sun, D., Jimenez, A. M., Lutkenhoff, E. S., Willhite, R., van Erp, T. G. and Cannon, T. D. (2008). Developmental disruptions in neural connectivity in the pathophysiology of schizophrenia. Dev Psychopathol 20, 1297-1327. 
Kato, M., Das, S., Petras, K., Kitamura, K., Morohashi, K., Abuelo, D. N., Barr, M., Bonneau, D., Brady, A. F., Carpenter, N. J., et al. (2004). Mutations of ARX are associated with striking pleiotropy and consistent genotype-phenotype correlation. Hum Mutat 23, 147-159.

Kato, M. and Dobyns, W. B. (2005). X-linked lissencephaly with abnormal genitalia as a tangential migration disorder causing intractable epilepsy: proposal for a new term, "interneuronopathy". J Child Neurol 20, 392-397.

Kavurma, M. M. and Khachigian, L. M. (2003). ERK, JNK, and p38 MAP kinases differentially regulate proliferation and migration of phenotypically distinct smooth muscle cell subtypes. J Cell Biochem 89, 289-300.

Kawaguchi, Y. and Kubota, Y. (1997). GABAergic cell subtypes and their synaptic connections in rat frontal cortex. Cereb Cortex 7, 476-486.

Kawasaki, A., Okada, M., Tamada, A., Okuda, S., Nozumi, M., Ito, Y., Kobayashi, D., Yamasaki, T., Yokoyama, R., Shibata, T., et al. (2018). Growth Cone Phosphoproteomics Reveals that GAP-43 Phosphorylated by JNK Is a Marker of Axon Growth and Regeneration. In iScience, pp. 190-203.

Kawauchi, T., Chihama, K., Nabeshima, Y. and Hoshino, M. (2003). The in vivo roles of STEF/Tiam1, Rac1 and JNK in cortical neuronal migration. Embo j 22, 4190-4201.

Kelkar, N., Delmotte, M. H., Weston, C. R., Barrett, T., Sheppard, B. J., Flavell, R. A. and Davis, R. J. (2003). Morphogenesis of the telencephalic commissure requires scaffold protein JNKinteracting protein 3 (JIP3). Proc Natl Acad Sci U S A 100, 9843-9848.

Kennedy, T. E., Serafini, T., de la Torre, J. R. and Tessier-Lavigne, M. (1994). Netrins are diffusible chemotropic factors for commissural axons in the embryonic spinal cord. Cell 78, 425-435.

Kennedy, T. E., Wang, H., Marshall, W. and Tessier-Lavigne, M. (2006). Axon guidance by diffusible chemoattractants: a gradient of netrin protein in the developing spinal cord. J Neurosci 26, 8866-8874.

Kidd, T., Bland, K. S. and Goodman, C. S. (1999). Slit is the midline repellent for the robo receptor in Drosophila. Cell 96, 785-794.

Killackey, H., Jacquin, M. and Rhoades, R. (1990). Development of somatosensory system structures. In: Development of sensory systems (Coleman Jr, ed), pp403-429. New York: Wiley.

Kim, H., Jung, O., Kang, M., Lee, M. S., Jeong, D., Ryu, J., Ko, Y., Choi, Y. J. and Lee, J. W. (2012). JNK signaling activity regulates cell-cell adhesions via TM4SF5-mediated p27(Kip1) phosphorylation. Cancer Lett 314, 198-205.

Kitamura, K., Yanazawa, M., Sugiyama, N., Miura, H., lizuka-Kogo, A., Kusaka, M., Omichi, K., Suzuki, R., Kato-Fukui, Y., Kamiirisa, K., et al. (2002). Mutation of ARX causes abnormal development of forebrain and testes in mice and X-linked lissencephaly with abnormal genitalia in humans. Nat Genet 32, 359-369.

Kleinhans, N. M., Richards, T., Sterling, L., Stegbauer, K. C., Mahurin, R., Johnson, L. C., Greenson, J., Dawson, G. and Aylward, E. (2008). Abnormal functional connectivity in autism spectrum disorders during face processing. Brain 131, 1000-1012.

Kuan, C. Y., Yang, D. D., Samanta Roy, D. R., Davis, R. J., Rakic, P. and Flavell, R. A. (1999). The Jnk1 and Jnk2 protein kinases are required for regional specific apoptosis during early brain development. Neuron 22, 667-676.

Kunde, S. A., Rademacher, N., Tzschach, A., Wiedersberg, E., Ullmann, R., Kalscheuer, V. M. and Shoichet, S. A. (2013). Characterisation of de novo MAPK10/JNK3 truncation mutations associated with cognitive disorders in two unrelated patients. Hum Genet 132, 461-471.

Kyriakis, J. M. and Avruch, J. (1990). pp54 microtubule-associated protein 2 kinase. A novel serine/threonine protein kinase regulated by phosphorylation and stimulated by poly-L-lysine. $J$ Biol Chem 265, 17355-17363. 
Kyriakis, J. M., Banerjee, P., Nikolakaki, E., Dai, T., Rubie, E. A., Ahmad, M. F., Avruch, J. and Woodgett, J. R. (1994). The stress-activated protein kinase subfamily of c-Jun kinases. Nature 369, 156-160.

Lakhina, V., Falnikar, A., Bhatnagar, L. and Tole, S. (2007). Early thalamocortical tract guidance and topographic sorting of thalamic projections requires LIM-homeodomain gene Lhx2. Dev Biol 306, 703-713.

Larsen, R., Proue, A., Scott, E. P., Christiansen, M. and Nakagawa, Y. (2019). The Thalamus Regulates Retinoic Acid Signaling and Development of Parvalbumin Interneurons in Postnatal Mouse Prefrontal Cortex. eNeuro 6.

Lavdas, A. A., Grigoriou, M., Pachnis, V. and Parnavelas, J. G. (1999). The medial ganglionic eminence gives rise to a population of early neurons in the developing cerebral cortex. J Neurosci 19, 7881-7888.

Lee, S., Hjerling-Leffler, J., Zagha, E., Fishell, G. and Rudy, B. (2010). The largest group of superficial neocortical GABAergic interneurons expresses ionotropic serotonin receptors. J Neurosci 30, 16796-16808.

Leighton, P. A., Mitchell, K. J., Goodrich, L. V., Lu, X., Pinson, K., Scherz, P., Skarnes, W. C. and TessierLavigne, M. (2001). Defining brain wiring patterns and mechanisms through gene trapping in mice. Nature 410, 174-179.

Letzkus, J. J., Wolff, S. B., Meyer, E. M., Tovote, P., Courtin, J., Herry, C. and Luthi, A. (2011). A disinhibitory microcircuit for associative fear learning in the auditory cortex. Nature 480, 331335.

Leuret, F. and Gratiolet, L. (1839). Anatomie comparée du système nerveux, considéré dans ses rapports avec l'intelligence. Baillière, Paris.

Levitt, P. and Rakic, P. (1980). Immunoperoxidase localization of glial fibrillary acidic protein in radial glial cells and astrocytes of the developing rhesus monkey brain. J Comp Neurol 193, 815-840.

Leyva-Diaz, E., del Toro, D., Menal, M. J., Cambray, S., Susin, R., Tessier-Lavigne, M., Klein, R., Egea, J. and Lopez-Bendito, G. (2014). FLRT3 is a Robo1-interacting protein that determines Netrin-1 attraction in developing axons. Curr Biol 24, 494-508.

Leyva-Diaz, E. and Lopez-Bendito, G. (2013). In and out from the cortex: development of major forebrain connections. Neuroscience 254, 26-44.

Li, F., Chong, Z. Z. and Maiese, K. (2005). Vital elements of the Wnt-Frizzled signaling pathway in the nervous system. Curr Neurovasc Res 2, 331-340.

Li, G., Adesnik, H., Li, J., Long, J., Nicoll, R. A., Rubenstein, J. L. and Pleasure, S. J. (2008). Regional distribution of cortical interneurons and development of inhibitory tone are regulated by Cxcl12/Cxcr4 signaling. J Neurosci 28, 1085-1098.

Liao, Y. X., Fu, Z. Z., Zhou, C. H., Shan, L. C., Wang, Z. Y., Yin, F., Zheng, L. P., Hua, Y. Q. and Cai, Z. D. (2015). AMD3100 reduces CXCR4-mediated survival and metastasis of osteosarcoma by inhibiting JNK and Akt, but not p38 or Erk1/2, pathways in in vitro and mouse experiments. Oncol Rep 34, 33-42.

Little, G. E., Lopez-Bendito, G., Runker, A. E., Garcia, N., Pinon, M. C., Chedotal, A., Molnar, Z. and Mitchell, K. J. (2009). Specificity and plasticity of thalamocortical connections in Sema6A mutant mice. PLoS Biol 7, e98.

Lodato, S., Rouaux, C., Quast, K. B., Jantrachotechatchawan, C., Studer, M., Hensch, T. K. and Arlotta, P. (2011). Excitatory projection neuron subtypes control the distribution of local inhibitory interneurons in the cerebral cortex. Neuron 69, 763-779.

Lokmane, L., Proville, R., Narboux-Nême, N., Györy, I., Keita, M., Mailhes, C., Léna, C., Gaspar, P., Grosschedl, R. and Garel, S. (2013). Sensory Map Transfer to the Neocortex Relies on Pretarget Ordering of Thalamic Axons. Current biology : CB 23. 
Long, H., Sabatier, C., Ma, L., Plump, A., Yuan, W., Ornitz, D. M., Tamada, A., Murakami, F., Goodman, C. S. and Tessier-Lavigne, M. (2004). Conserved roles for Slit and Robo proteins in midline commissural axon guidance. Neuron 42, 213-223.

Lopez-Bendito, G. (2018). Development of the Thalamocortical Interactions: Past, Present and Future. Neuroscience 385, 67-74.

Lopez-Bendito, G., Cautinat, A., Sanchez, J. A., Bielle, F., Flames, N., Garratt, A. N., Talmage, D. A., Role, L. W., Charnay, P., Marin, O., et al. (2006). Tangential neuronal migration controls axon guidance: a role for neuregulin-1 in thalamocortical axon navigation. Cell 125, 127-142.

Lopez-Bendito, G., Chan, C. H., Mallamaci, A., Parnavelas, J. and Molnar, Z. (2002). Role of Emx2 in the development of the reciprocal connectivity between cortex and thalamus. J Comp Neurol 451, 153-169.

Lopez-Bendito, G., Flames, N., Ma, L., Fouquet, C., Di Meglio, T., Chedotal, A., Tessier-Lavigne, M. and Marin, O. (2007). Robo1 and Robo2 cooperate to control the guidance of major axonal tracts in the mammalian forebrain. J Neurosci 27, 3395-3407.

Lopez-Bendito, G. and Molnar, Z. (2003). Thalamocortical development: how are we going to get there? Nat Rev Neurosci 4, 276-289.

Lopez-Bendito, G., Sanchez-Alcaniz, J. A., Pla, R., Borrell, V., Pico, E., Valdeolmillos, M. and Marin, O. (2008). Chemokine signaling controls intracortical migration and final distribution of GABAergic interneurons. J Neurosci 28, 1613-1624.

Lopez-Bendito, G., Sturgess, K., Erdelyi, F., Szabo, G., Molnar, Z. and Paulsen, O. (2004). Preferential origin and layer destination of GAD65-GFP cortical interneurons. Cereb Cortex 14, 1122-1133.

Lund, R. D. and Mustari, M. J. (1977). Development of the geniculocortical pathway in rats. J Comp Neurol 173, 289-306.

Lysko, D. E., Putt, M. and Golden, J. A. (2011). SDF1 regulates leading process branching and speed of migrating interneurons. J Neurosci 31, 1739-1745.

---- (2014). SDF1 reduces interneuron leading process branching through dual regulation of actin and microtubules. J Neurosci 34, 4941-4962.

Ma, Y., Hu, H., Berrebi, A. S., Mathers, P. H. and Agmon, A. (2006). Distinct subtypes of somatostatincontaining neocortical interneurons revealed in transgenic mice. J Neurosci 26, 5069-5082.

Malchinkhuu, E., Sato, K., Horiuchi, Y., Mogi, C., Ohwada, S., Ishiuchi, S., Saito, N., Kurose, H., Tomura, H. and Okajima, F. (2005). Role of p38 mitogen-activated kinase and c-Jun terminal kinase in migration response to lysophosphatidic acid and sphingosine-1-phosphate in glioma cells. Oncogene 24, 6676-6688.

Mandai, K., Reimert, D. V. and Ginty, D. D. (2014). Linx mediates interaxonal interactions and formation of the internal capsule. Neuron 83, 93-103.

Manent, J. B., Jorquera, I., Ben-Ari, Y., Aniksztejn, L. and Represa, A. (2006). Glutamate acting on AMPA but not NMDA receptors modulates the migration of hippocampal interneurons. J Neurosci 26, 5901-5909.

Manning, A. M. and Davis, R. J. (2003). Targeting JNK for therapeutic benefit: from junk to gold? Nat Rev Drug Discov 2, 554-565.

Marcos-Mondejar, P., Peregrin, S., Li, J. Y., Carlsson, L., Tole, S. and Lopez-Bendito, G. (2012). The Ihx2 transcription factor controls thalamocortical axonal guidance by specific regulation of robo1 and robo2 receptors. J Neurosci 32, 4372-4385.

Marcus, R., Blazeski, R., Godement, P. and Mason, C. (1995). Retinal Axon Divergence in the Optic Chiasm: Uncrossed Axons Diverge From Crossed Axons Within a Midline Glial Specialization. The Journal of Neuroscience 15.

Marin, O. (2012). Interneuron dysfunction in psychiatric disorders. Nat Rev Neurosci 13, 107-120. 
---- (2013). Cellular and molecular mechanisms controlling the migration of neocortical interneurons. Eur J Neurosci 38, 2019-2029.

Marin, O., Yaron, A., Bagri, A., Tessier-Lavigne, M. and Rubenstein, J. L. (2001). Sorting of striatal and cortical interneurons regulated by semaphorin-neuropilin interactions. Science 293, 872-875.

Marinissen, M. J., Chiariello, M., Tanos, T., Bernard, O., Narumiya, S. and Gutkind, J. S. (2004). The small GTP-binding protein RhoA regulates c-jun by a ROCK-JNK signaling axis. Mol Cell 14, 29-41.

Markram, H., Toledo-Rodriguez, M., Wang, Y., Gupta, A., Silberberg, G. and Wu, C. (2004). Interneurons of the neocortical inhibitory system. Nat Rev Neurosci 5, 793-807.

Martin, J. H., Mohit, A. A. and Miller, C. A. (1996). Developmental expression in the mouse nervous system of the p493F12 SAP kinase. Brain Res Mol Brain Res 35, 47-57.

Martini, F. J. and Valdeolmillos, M. (2010). Actomyosin contraction at the cell rear drives nuclear translocation in migrating cortical interneurons. J Neurosci 30, 8660-8670.

Marín, O. and Müller, U. (2014). Lineage Origins of GABAergic Versus Glutamatergic Neurons in the Neocortex. Current opinion in neurobiology 26.

Mazzitelli, S., Xu, P., Ferrer, I., Davis, R. J. and Tournier, C. (2011). The loss of c-Jun N-terminal protein kinase activity prevents the amyloidogenic cleavage of amyloid precursor protein and the formation of amyloid plaques in vivo. J Neurosci 31, 16969-16976.

McFadden, K. and Minshew, N. J. (2013). Evidence for dysregulation of axonal growth and guidance in the etiology of ASD. Front Hum Neurosci 7, 671.

McIntosh, A. R. (2000). Towards a network theory of cognition. Neural Netw 13, 861-870.

McManus, M. F., Nasrallah, I. M., Gopal, P. P., Baek, W. S. and Golden, J. A. (2004). Axon mediated interneuron migration. J Neuropathol Exp Neurol 63, 932-941.

Meechan, D. W., Rutz, H. L., Fralish, M. S., Maynard, T. M., Rothblat, L. A. and LaMantia, A. S. (2015). Cognitive ability is associated with altered medial frontal cortical circuits in the LgDel mouse model of 22q11.2DS. Cereb Cortex 25, 1143-1151.

Meechan, D. W., Tucker, E. S., Maynard, T. M. and LaMantia, A.-S. (2012a). Cxcr4 regulation of interneuron migration is disrupted in 22q11.2 deletion syndrome. Proceedings of the National Academy of Sciences of the United States of America 109, 18601-18606.

Meechan, D. W., Tucker, E. S., Maynard, T. M. and LaMantia, A. S. (2009). Diminished dosage of 22q11 genes disrupts neurogenesis and cortical development in a mouse model of 22q11 deletion/DiGeorge syndrome. Proc Natl Acad Sci U S A 106, 16434-16445.

---- (2012b). Cxcr4 regulation of interneuron migration is disrupted in 22q11.2 deletion syndrome. Proc Natl Acad Sci U S A 109, 18601-18606.

Meinecke, D. L. and Peters, A. (1987). GABA immunoreactive neurons in rat visual cortex. J Comp Neurol 261, 388-404.

Metin, C., Deleglise, D., Serafini, T., Kennedy, T. E. and Tessier-Lavigne, M. (1997). A role for netrin-1 in the guidance of cortical efferents. Development 124, 5063-5074.

Metin, C., Denizot, J. P. and Ropert, N. (2000). Intermediate zone cells express calcium-permeable AMPA receptors and establish close contact with growing axons. J Neurosci 20, 696-708.

Metin, C. and Godement, P. (1996). The ganglionic eminence may be an intermediate target for corticofugal and thalamocortical axons. J Neurosci 16, 3219-3235.

Miller, M. W. (1985). Cogeneration of retrogradely labeled corticocortical projection and GABAimmunoreactive local circuit neurons in cerebral cortex. Brain Res 355, 187-192.

Minden, A., Lin, A., Claret, F. X., Abo, A. and Karin, M. (1995). Selective activation of the JNK signaling cascade and c-Jun transcriptional activity by the small GTPases Rac and Cdc42Hs. Cell 81, 11471157. 
Misic, B., Betzel, R. F., de Reus, M. A., van den Heuvel, M. P., Berman, M. G., McIntosh, A. R. and Sporns, O. (2016). Network-Level Structure-Function Relationships in Human Neocortex. Cereb Cortex 26, 3285-3296.

Mitrofanis, J. (1994). Development of the pathway from the reticular and perireticular nuclei to the thalamus in ferrets: a Dil study. Eur J Neurosci 6, 1864-1882.

Mitrofanis, J. and Guillery, R. W. (1993). New views of the thalamic reticular nucleus in the adult and the developing brain. Trends Neurosci 16, 240-245.

Mitsogiannis, M. D., Little, G. E. and Mitchell, K. J. (2017). Semaphorin-Plexin signaling influences early ventral telencephalic development and thalamocortical axon guidance. Neural Dev 12, 6.

Miyata, T., Kawaguchi, A., Saito, K., Kawano, M., Muto, T. and Ogawa, M. (2004). Asymmetric production of surface-dividing and non-surface-dividing cortical progenitor cells. Development 131, 3133-3145.

Miyoshi, G., Butt, S. J., Takebayashi, H. and Fishell, G. (2007). Physiologically distinct temporal cohorts of cortical interneurons arise from telencephalic Olig2-expressing precursors. J Neurosci 27, 7786-7798.

Miyoshi, G. and Fishell, G. (2011). GABAergic interneuron lineages selectively sort into specific cortical layers during early postnatal development. Cereb Cortex 21, 845-852.

Miyoshi, G., Hjerling-Leffler, J., Karayannis, T., Sousa, V. H., Butt, S. J., Battiste, J., Johnson, J. E., Machold, R. P. and Fishell, G. (2010). Genetic fate mapping reveals that the caudal ganglionic eminence produces a large and diverse population of superficial cortical interneurons. $J$ Neurosci 30, 1582-1594.

Mohammad, H., Marchisella, F., Ortega-Martinez, S., Hollos, P., Eerola, K., Komulainen, E., Kulesskaya, N., Freemantle, E., Fagerholm, V., Savontous, E., et al. (2018). JNK1 controls adult hippocampal neurogenesis and imposes cell-autonomous control of anxiety behaviour from the neurogenic niche. Mol Psychiatry 23, 487.

Molnar, Z., Adams, R. and Blakemore, C. (1998). Mechanisms underlying the early establishment of thalamocortical connections in the rat. J Neurosci 18, 5723-5745.

Molnar, Z. and Blakemore, C. (1995). How do thalamic axons find their way to the cortex? Trends Neurosci 18, 389-397.

Molnar, Z. and Cordery, P. (1999). Connections between cells of the internal capsule, thalamus, and cerebral cortex in embryonic rat. J Comp Neurol 413, 1-25.

Molnar, Z., Garel, S., Lopez-Bendito, G., Maness, P. and Price, D. J. (2012). Mechanisms controlling the guidance of thalamocortical axons through the embryonic forebrain. Eur J Neurosci 35, 15731585.

Morales, B., Choi, S.-Y. and Kirkwood, A. (2002). Dark Rearing Alters the Development of GABAergic Transmission in Visual Cortex. The Journal of neuroscience : the official journal of the Society for Neuroscience 22.

Morello, F., Prasad, A. A., Rehberg, K., Vieira de Sa, R., Anton-Bolanos, N., Leyva-Diaz, E., Adolfs, Y., Tissir, F., Lopez-Bendito, G. and Pasterkamp, R. J. (2015). Frizzled3 Controls Axonal Polarity and Intermediate Target Entry during Striatal Pathway Development. J Neurosci 35, 14205-14219.

Morest, D. K. (1970). A study of neurogenesis in the forebrain of opossum pouch young. $Z$ Anat Entwicklungsgesch 130, 265-305.

Mountcastle, V. B. (1957). Modality and topographic properties of single neurons of cat's somatic sensory cortex. J Neurophysiol 20, 408-434.

Moya, F. and Valdeolmillos, M. (2004). Polarized increase of calcium and nucleokinesis in tangentially migrating neurons. Cereb Cortex 14, 610-618. 
Myers, A. K., Cunningham, J. G., Smith, S. E., Snow, J. P., Smoot, C. A. and Tucker, E. S. (2020). JNK signaling is required for proper tangential migration and laminar allocation of cortical interneurons. Development 147.

Myers, A. K., Meechan, D. W., Adney, D. R. and Tucker, E. S. (2014). Cortical interneurons require Jnk1 to enter and navigate the developing cerebral cortex. J Neurosci 34, 7787-7801.

Nadarajah, B., Alifragis, P., Wong, R. O. and Parnavelas, J. G. (2003). Neuronal migration in the developing cerebral cortex: observations based on real-time imaging. Cereb Cortex 13, 607-611.

Nadarajah, B. and Parnavelas, J. G. (2002). Modes of neuronal migration in the developing cerebral cortex. Nat Rev Neurosci 3, 423-432.

Nair, A., Treiber, J. M., Shukla, D. K., Shih, P. and Muller, R. A. (2013). Impaired thalamocortical connectivity in autism spectrum disorder: a study of functional and anatomical connectivity. Brain 136, 1942-1955.

Nakajima, M. and Halassa, M. M. (2017). Thalamic control of functional cortical connectivity. Curr Opin Neurobiol 44, 127-131.

Nakashiba, T., Ikeda, T., Nishimura, S., Tashiro, K., Honjo, T., Culotti, J. G. and Itohara, S. (2000). Netrin-G1: a novel glycosyl phosphatidylinositol-linked mammalian netrin that is functionally divergent from classical netrins. J Neurosci 20, 6540-6550.

Naruse, M., Ishizaki, Y., Ikenaka, K., Tanaka, A. and Hitoshi, S. (2017). Origin of oligodendrocytes in mammalian forebrains: a revised perspective. In J Physiol Sci, pp. 63-70.

Nave, K.-A. (2010). Myelination and Support of Axonal Integrity by Glia. Nature 468.

Nery, S., Fishell, G. and Corbin, J. G. (2002). The caudal ganglionic eminence is a source of distinct cortical and subcortical cell populations. Nat Neurosci 5, 1279-1287.

Neyt, C., Welch, M., Langston, A., Kohtz, J. and Fishell, G. (1997). A Short-Range Signal Restricts Cell Movement Between Telencephalic Proliferative Zones. The Journal of neuroscience : the official journal of the Society for Neuroscience 17.

Niehrs, C. (2012). The complex world of WNT receptor signalling. Nat Rev Mol Cell Biol 13, 767-779.

Niklasson, L., Rasmussen, P., Oskarsdottir, S. and Gillberg, C. (2009). Autism, ADHD, mental retardation and behavior problems in 100 individuals with 22q11 deletion syndrome. Res Dev Disabil 30, 763-773.

Nishikimi, M., Oishi, K. and Nakajima, K. (2013). Axon Guidance Mechanisms for Establishment of Callosal Connections. Neural plasticity 2013.

Nishimura, Y. V., Sekine, K., Chihama, K., Nakajima, K., Hoshino, M., Nabeshima, Y. and Kawauchi, T. (2010). Dissecting the factors involved in the locomotion mode of neuronal migration in the developing cerebral cortex. J Biol Chem 285, 5878-5887.

Nishimura, Y. V., Shikanai, M., Hoshino, M., Ohshima, T., Nabeshima, Y., Mizutani, K., Nagata, K., Nakajima, K. and Kawauchi, T. (2014). Cdk5 and its substrates, Dcx and p27kip1, regulate cytoplasmic dilation formation and nuclear elongation in migrating neurons. Development 141, 3540-3550.

Noctor, S. C., Flint, A. C., Weissman, T. A., Dammerman, R. S. and Kriegstein, A. R. (2001). Neurons derived from radial glial cells establish radial units in neocortex. Nature 409, 714-720.

O'Rourke, N. A., Dailey, M. E., Smith, S. J. and McConnell, S. K. (1992). Diverse migratory pathways in the developing cerebral cortex. Science 258, 299-302.

Ogata, M., Hino, S., Saito, A., Morikawa, K., Kondo, S., Kanemoto, S., Murakami, T., Taniguchi, M., Tanii, I., Yoshinaga, K., et al. (2006). Autophagy is activated for cell survival after endoplasmic reticulum stress. Mol Cell Biol 26, 9220-9231.

Oliva, A. A., Jr., Atkins, C. M., Copenagle, L. and Banker, G. A. (2006). Activated c-Jun N-terminal kinase is required for axon formation. J Neurosci 26, 9462-9470. 
Ono, K., Clavairoly, A., Nomura, T., Gotoh, H., Uno, A., Armant, O., Takebayashi, H., Zhang, Q., Shimamura, K., Itohara, S., et al. (2014). Development of the prethalamus is crucial for thalamocortical projection formation and is regulated by Olig2. Development 141, 2075-2084.

Panganiban, G. and Rubenstein, J. L. (2002). Developmental functions of the Distal-less/Dlx homeobox genes. Development 129, 4371-4386.

Pantelis, C., Yucel, M., Wood, S. J., McGorry, P. D. and Velakoulis, D. (2003). Early and late neurodevelopmental disturbances in schizophrenia and their functional consequences. Aust NZ J Psychiatry 37, 399-406.

Patel, P. and Moshe, S. L. (2020). The evolution of the concepts of seizures and epilepsy: What's in a name? Epilepsia Open 5, 22-35.

Pavlowsky, A., Gianfelice, A., Pallotto, M., Zanchi, A., Vara, H., Khelfaoui, M., Valnegri, P., Rezai, X., Bassani, S., Brambilla, D., et al. (2010a). A postsynaptic signaling pathway that may account for the cognitive defect due to IL1RAPL1 mutation. Curr Biol 20, 103-115.

Pavlowsky, A., Zanchi, A., Pallotto, M., Giustetto, M., Chelly, J., Sala, C. and Billuart, P. (2010b). Neuronal JNK pathway activation by IL-1 is mediated through IL1RAPL1, a protein required for development of cognitive functions. In Commun Integr Biol, pp. 245-247.

Pei, J. J., Braak, E., Braak, H., Grundke-Iqbal, I., Iqbal, K., Winblad, B. and Cowburn, R. F. (2001). Localization of active forms of C-jun kinase (JNK) and p38 kinase in Alzheimer's disease brains at different stages of neurofibrillary degeneration. J Alzheimers Dis 3, 41-48.

Pessoa, L. and Hof, P. R. (2015). From Paul Broca's great limbic lobe to the limbic system. J Comp Neurol 523, 2495-2500.

Petrich, B. G., Gong, X., Lerner, D. L., Wang, X., Brown, J. H., Saffitz, J. E. and Wang, Y. (2002). c-Jun Nterminal kinase activation mediates downregulation of connexin43 in cardiomyocytes. Circ Res 91, 640-647.

Piton, A., Michaud, J. L., Peng, H., Aradhya, S., Gauthier, J., Mottron, L., Champagne, N., Lafreniere, R. G., Hamdan, F. F., Joober, R., et al. (2008). Mutations in the calcium-related gene IL1RAPL1 are associated with autism. Hum Mol Genet 17, 3965-3974.

Pla, R., Borrell, V., Flames, N. and Marin, O. (2006). Layer acquisition by cortical GABAergic interneurons is independent of Reelin signaling. J Neurosci 26, 6924-6934.

Polleux, F. and Ghosh, A. (2002). The slice overlay assay: a versatile tool to study the influence of extracellular signals on neuronal development. SCi STKE 2002, pl9.

Poluch, S. and Juliano, S. L. (2007). A normal radial glial scaffold is necessary for migration of interneurons during neocortical development. Glia 55, 822-830.

Pont-Lezica, L., Beumer, W., Colasse, S., Drexhage, H., Versnel, M. and Bessis, A. (2014). Microglia Shape Corpus Callosum Axon Tract Fasciculation: Functional Impact of Prenatal Inflammation. The European journal of neuroscience 39.

Powell, A. W., Sassa, T., Wu, Y., Tessier-Lavigne, M. and Polleux, F. (2008). Topography of thalamic projections requires attractive and repulsive functions of Netrin-1 in the ventral telencephalon. PLoS Biol 6, e116.

Powell, E. M., Mars, W. M. and Levitt, P. (2001). Hepatocyte growth factor/scatter factor is a motogen for interneurons migrating from the ventral to dorsal telencephalon. Neuron 30, 79-89.

Powell, E. M., Muhlfriedel, S., Bolz, J. and Levitt, P. (2003). Differential regulation of thalamic and cortical axonal growth by hepatocyte growth factor/scatter factor. Dev Neurosci 25, 197-206.

Pulsipher, D. T., Dabbs, K., Tuchsherer, V., Sheth, R. D., Koehn, M. A., Hermann, B. P. and Seidenberg, M. (2011). Thalamofrontal neurodevelopment in new-onset pediatric idiopathic generalized epilepsy. Neurology 76, 28-33.

Qiu, M. G., Ye, Z., Li, Q. Y., Liu, G. J., Xie, B. and Wang, J. (2011). Changes of brain structure and function in ADHD children. Brain Topogr 24, 243-252. 
Qu, C., Li, W., Shao, Q., Dwyer, T., Huang, H., Yang, T. and Liu, G. (2013). c-Jun N-terminal kinase 1 (JNK1) is required for coordination of netrin signaling in axon guidance. J Biol Chem 288, 18831895.

Qu, Y., Huang, Y., Feng, J., Alvarez-Bolado, G., Grove, E. A., Yang, Y., Tissir, F., Zhou, L. and Goffinet, A. M. (2014). Genetic evidence that Celsr3 and Celsr2, together with Fzd3, regulate forebrain wiring in a Vangl-independent manner. In Proc Natl Acad Sci U S A, pp. E2996-3004.

Raivich, G., Bohatschek, M., Da Costa, C., Iwata, O., Galiano, M., Hristova, M., Nateri, A. S., Makwana, M., Riera-Sans, L., Wolfer, D. P., et al. (2004). The AP-1 transcription factor c-Jun is required for efficient axonal regeneration. Neuron 43, 57-67.

Rakic, P. (1972). Mode of cell migration to the superficial layers of fetal monkey neocortex. J Comp Neurol 145, 61-83.

---- (1977). Genesis of the dorsal lateral geniculate nucleus in the rhesus monkey: site and time of origin, kinetics of proliferation, routes of migration and pattern of distribution of neurons. J Comp Neurol 176, 23-52.

---- (2007). The radial edifice of cortical architecture: from neuronal silhouettes to genetic engineering. Brain Res Rev 55, 204-219.

Ramon y Cajal, S. (1881). Sur la structure de l'ecorce cerebrale de quelques mammiferes. La Cellule.

---- (1895). Histology of the Nervous System, trans.: Swanson, N. and Swanson, L.W. Oxford: Oxford University Press, 805.

Reemst, K., Noctor, S. C., Lucassen, P. J. and Hol, E. M. (2016). The Indispensable Roles of Microglia and Astrocytes During Brain Development. Frontiers in human neuroscience 10.

Reynolds, C. H., Utton, M. A., Gibb, G. M., Yates, A. and Anderton, B. H. (1997). Stress-activated protein kinase/c-jun N-terminal kinase phosphorylates tau protein. J Neurochem 68, 1736-1744.

Rice, F., Gomez, C., Barstow, C., Burnet, A. and Sands, P. (1985). A Comparative Analysis of the Development of the Primary Somatosensory Cortex: Interspecies Similarities During Barrel and Laminar Development. The Journal of comparative neurology 236.

Riedemann, T. (2019). Diversity and Function of Somatostatin-Expressing Interneurons in the Cerebral Cortex. Int J Mol Sci 20.

Rigby, M. J., Gomez, T. M. and Puglielli, L. (2020). Glial Cell-Axonal Growth Cone Interactions in Neurodevelopment and Regeneration. Frontiers in neuroscience 14.

Rossignol, E. (2011). Genetics and function of neocortical GABAergic interneurons in neurodevelopmental disorders. Neural Plast 2011, 649325.

Rosso, S. B., Sussman, D., Wynshaw-Boris, A. and Salinas, P. C. (2005). Wnt signaling through Dishevelled, Rac and JNK regulates dendritic development. Nat Neurosci 8, 34-42.

Rubenstein, J. L. R. and Merzenich, M. M. (2003). Model of autism: increased ratio of excitation/inhibition in key neural systems. Genes Brain Behav 2, 255-267.

Rudolph, J., Zimmer, G., Steinecke, A., Barchmann, S. and Bolz, J. (2010). Ephrins guide migrating cortical interneurons in the basal telencephalon. Cell Adh Migr 4, 400-408.

Rudy, B., Fishell, G., Lee, S. and Hjerling-Leffler, J. (2011). Three groups of interneurons account for nearly $100 \%$ of neocortical GABAergic neurons. Dev Neurobiol 71, 45-61.

Sabapathy, K., Jochum, W., Hochedlinger, K., Chang, L., Karin, M. and Wagner, E. F. (1999). Defective neural tube morphogenesis and altered apoptosis in the absence of both JNK1 and JNK2. Mech Dev 89, 115-124.

Salek-Haddadi, A., Lemieux, L., Merschhemke, M., Friston, K. J., Duncan, J. S. and Fish, D. R. (2003). Functional magnetic resonance imaging of human absence seizures. Ann Neurol 53, 663-667.

Sanchez-Alcaniz, J. A., Haege, S., Mueller, W., Pla, R., Mackay, F., Schulz, S., Lopez-Bendito, G., Stumm, R. and Marin, O. (2011). Cxcr7 controls neuronal migration by regulating chemokine responsiveness. Neuron $69,77-90$. 
Scholpp, S. and Lumsden, A. (2010). Building a bridal chamber: development of the thalamus. Trends Neurosci 33, 373-380.

Serafini, T., Colamarino, S. A., Leonardo, E. D., Wang, H., Beddington, R., Skarnes, W. C. and TessierLavigne, M. (1996). Netrin-1 is required for commissural axon guidance in the developing vertebrate nervous system. Cell 87, 1001-1014.

Shatz, C. J. and Luskin, M. B. (1986). The relationship between the geniculocortical afferents and their cortical target cells during development of the cat's primary visual cortex. J Neurosci 6, 36553668.

Shenton, M. E., Dickey, C. C., Frumin, M. and McCarley, R. W. (2001). A review of MRI findings in schizophrenia. Schizophr Res 49, 1-52.

Sherman, S. M. (2017). Functioning of Circuits Connecting Thalamus and Cortex. Compr Physiol 7, 713739.

Sherman, S. M. and Guillery, R. W. (2011). Distinct functions for direct and transthalamic corticocortical connections. J Neurophysiol 106, 1068-1077.

Shoichet, S. A., Duprez, L., Hagens, O., Waetzig, V., Menzel, C., Herdegen, T., Schweiger, S., Dan, B., Vamos, E., Ropers, H. H., et al. (2006). Truncation of the CNS-expressed JNK3 in a patient with a severe developmental epileptic encephalopathy. Hum Genet 118, 559-567.

Shoukimas, G. M. and Hinds, J. W. (1978). The development of the cerebral cortex in the embryonic mouse: an electron microscopic serial section analysis. J Comp Neurol 179, 795-830.

Shu, T., Puche, A. C. and Richards, L. J. (2003). Development of Midline Glial Populations at the Corticoseptal Boundary. Journal of neurobiology 57.

Shu, T. and Richards, L. J. (2001). Cortical Axon Guidance by the Glial Wedge During the Development of the Corpus Callosum. The Journal of Neuroscience 21.

Silver, J., Lorenz, S., Wahlsten, D. and Coughlin, J. (1982). Axonal Guidance During Development of the Great Cerebral Commissures: Descriptive and Experimental Studies, in Vivo, on the Role of Preformed Glial Pathways. The Journal of comparative neurology 210.

Simons, M. and Mlodzik, M. (2008). Planar cell polarity signaling: from fly development to human disease. Annu Rev Genet 42, 517-540.

Simpson, T. I., Pratt, T., Mason, J. O. and Price, D. J. (2009). Normal ventral telencephalic expression of Pax6 is required for normal development of thalamocortical axons in embryonic mice. Neural Dev 4, 19.

Smith, S. E., Coker, N. K. and Tucker, E. S. (2020). JNK signaling controls branching, nucleokinesis, and positioning of centrosomes and primary cilia in migrating cortical interneurons. bioRxiv, 2020.2001.2030.927855.

Soria, J. M., Martinez-Galan, J. R., Lujan, R., Valdeolmillos, M. and Fairen, A. (1999). Functional NMDA and GABAA receptors in pioneer neurons of the cortical marginal zone. Eur J Neurosci 11, 33513354.

Squarzoni, P., Oller, G., Hoeffel, G., Pont-Lezica, L., Rostaing, P., Low, D., Bessis, A., Ginhoux, F. and Garel, S. (2014). Microglia modulate wiring of the embryonic forebrain. Cell Rep 8, 1271-1279.

Squarzoni, P., Thion, M. S. and Garel, S. (2015). Neuronal and microglial regulators of cortical wiring: usual and novel guideposts. Front Neurosci 9, 248.

Stallcup, W. B., Beasley, L. L. and Levine, J. M. (1985). Antibody against nerve growth factor-inducible large external (NILE) glycoprotein labels nerve fiber tracts in the developing rat nervous system. J Neurosci 5, 1090-1101.

Stenman, J., Toresson, H. and Campbell, K. (2003). Identification of two distinct progenitor populations in the lateral ganglionic eminence: implications for striatal and olfactory bulb neurogenesis. $J$ Neurosci 23, 167-174. 
Stensaas, L. J. (1967). The development of hippocampal and dorsolateral pallial regions of the cerebral hemisphere in fetal rabbits. IV. Forty-one millimeter stage, intermediate lamina. J Comp Neurol 131, 409-422.

Stoeckli, E. T. (2018). Understanding axon guidance: are we nearly there yet? Development 145.

Stromme, P., Mangelsdorf, M. E., Shaw, M. A., Lower, K. M., Lewis, S. M., Bruyere, H., Lutcherath, V., Gedeon, A. K., Wallace, R. H., Scheffer, I. E., et al. (2002). Mutations in the human ortholog of Aristaless cause X-linked mental retardation and epilepsy. Nat Genet 30, 441-445.

Stumm, R. K., Zhou, C., Ara, T., Lazarini, F., Dubois-Dalcq, M., Nagasawa, T., Hollt, V. and Schulz, S. (2003). CXCR4 regulates interneuron migration in the developing neocortex. J Neurosci 23, 51235130.

Suga, N. and Ma, X. (2003). Multiparametric Corticofugal Modulation and Plasticity in the Auditory System. Nature reviews. Neuroscience 4.

Sugimura, R. and Li, L. (2010). Noncanonical Wnt signaling in vertebrate development, stem cells, and diseases. Birth Defects Res C Embryo Today 90, 243-256.

Sussel, L., Marin, O., Kimura, S. and Rubenstein, J. L. (1999). Loss of Nkx2.1 homeobox gene function results in a ventral to dorsal molecular respecification within the basal telencephalon: evidence for a transformation of the pallidum into the striatum. Development 126, 3359-3370.

Talebian, A., Britton, R., Ammanuel, S., Bepari, A., Sprouse, F., Birnbaum, S. G., Szabo, G., Tamamaki, N., Gibson, J. and Henkemeyer, M. (2017). Autonomous and non-autonomous roles for ephrin$\mathrm{B}$ in interneuron migration. Dev Biol 431, 179-193.

Tamamaki, N., Fujimori, K. E. and Takauji, R. (1997). Origin and route of tangentially migrating neurons in the developing neocortical intermediate zone. J Neurosci 17, 8313-8323.

Tan, S. S. and Breen, S. (1993). Radial mosaicism and tangential cell dispersion both contribute to mouse neocortical development. Nature 362, 638-640.

Tan, X. and Shi, S. H. (2013). Neocortical neurogenesis and neuronal migration. Wiley Interdiscip Rev Dev Biol 2, 443-459.

Tang, L., Dai, F., Liu, Y., Yu, X., Huang, C., Wang, Y. and Yao, W. (2018). RhoA/ROCK signaling regulates smooth muscle phenotypic modulation and vascular remodeling via the JNK pathway and vimentin cytoskeleton. Pharmacol Res 133, 201-212.

Tararuk, T., Ostman, N., Li, W., Bjorkblom, B., Padzik, A., Zdrojewska, J., Hongisto, V., Herdegen, T., Konopka, W., Courtney, M. J., et al. (2006). JNK1 phosphorylation of SCG10 determines microtubule dynamics and axodendritic length. J Cell Biol 173, 265-277.

Tatti, R., Haley, M. S., Swanson, O., Tselha, T. and Maffei, A. (2017). Neurophysiology and regulation of the balance between excitation and inhibition in neocortical circuits. Biol Psychiatry 81, 821-831.

Tham, T. N., Lazarini, F., Franceschini, I. A., Lachapelle, F., Amara, A. and Dubois-Dalcq, M. (2001). Developmental pattern of expression of the alpha chemokine stromal cell-derived factor 1 in the rat central nervous system. Eur J Neurosci 13, 845-856.

Theyel, B. B., Llano, D. A. and Sherman, S. M. (2010). The corticothalamocortical circuit drives higherorder cortex in the mouse. Nat Neurosci 13, 84-88.

Tissir, F., Bar, I., Jossin, Y., De Backer, O. and Goffinet, A. M. (2005). Protocadherin Celsr3 is crucial in axonal tract development. Nat Neurosci 8, 451-457.

Tiveron, M. C., Rossel, M., Moepps, B., Zhang, Y. L., Seidenfaden, R., Favor, J., Konig, N. and Cremer, H. (2006). Molecular interaction between projection neuron precursors and invading interneurons via stromal-derived factor 1 (CXCL12)/CXCR4 signaling in the cortical subventricular zone/intermediate zone. J Neurosci 26, 13273-13278.

Trujillo, C. M., Alonso, A., Delgado, A. C. and Damas, C. (2005). The Rostral and Caudal Boundaries of the Diencephalon. Brain research. Brain research reviews 49. 
Tucker, E. S., Polleux, F. and LaMantia, A. S. (2006). Position and time specify the migration of a pioneering population of olfactory bulb interneurons. Dev Biol 297, 387-401.

Tuttle, R., Nakagawa, Y., Johnson, J. E. and O'Leary, D. D. (1999). Defects in thalamocortical axon pathfinding correlate with altered cell domains in Mash-1-deficient mice. Development 126, 1903-1916.

Uemura, M., Nakao, S., Suzuki, S. T., Takeichi, M. and Hirano, S. (2007). OL-Protocadherin is essential for growth of striatal axons and thalamocortical projections. Nat Neurosci 10, 1151-1159.

Usui, T., Shima, Y., Shimada, Y., Hirano, S., Burgess, R. W., Schwarz, T. L., Takeichi, M. and Uemura, T. (1999). Flamingo, a seven-pass transmembrane cadherin, regulates planar cell polarity under the control of Frizzled. Cell 98, 585-595.

Uzunova, G., Pallanti, S. and Hollander, E. (2016). Excitatory/inhibitory imbalance in autism spectrum disorders: Implications for interventions and therapeutics. World J Biol Psychiatry 17, 174-186.

Valcanis, H. and Tan, S. S. (2003). Layer specification of transplanted interneurons in developing mouse neocortex. J Neurosci 23, 5113-5122.

Vaughen, J. and Igaki, T. (2016). Slit-Robo Repulsive Signaling Extrudes Tumorigenic Cells from Epithelia. Dev Cell 39, 683-695.

Vinson, C. R., Conover, S. and Adler, P. N. (1989). A Drosophila tissue polarity locus encodes a protein containing seven potential transmembrane domains. Nature 338, 263-264.

Volk, D. W., Chitrapu, A., Edelson, J. R. and Lewis, D. A. (2015). Chemokine receptors and cortical interneuron dysfunction in schizophrenia. Schizophr Res 167, 12-17.

Vue, T. Y., Lee, M., Tan, Y. E., Werkhoven, Z., Wang, L. and Nakagawa, Y. (2013). Thalamic control of neocortical area formation in mice. J Neurosci 33, 8442-8453.

Walsh, C. and Cepko, C. L. (1988). Clonally related cortical cells show several migration patterns. Science 241, 1342-1345.

Wang, X., Nadarajah, B., Robinson, A. C., McColl, B. W., Jin, J. W., Dajas-Bailador, F., Boot-Handford, R. P. and Tournier, C. (2007). Targeted deletion of the mitogen-activated protein kinase kinase 4 gene in the nervous system causes severe brain developmental defects and premature death. Mol Cell Biol 27, 7935-7946.

Wang, Y., Li, G., Stanco, A., Long, J. E., Crawford, D., Potter, G. B., Pleasure, S. J., Behrens, T. and Rubenstein, J. L. (2011). CXCR4 and CXCR7 have distinct functions in regulating interneuron migration. Neuron 69, 61-76.

Wang, Y. and Nathans, J. (2007). Tissue/planar cell polarity in vertebrates: new insights and new questions. Development 134, 647-658.

Wang, Y., Thekdi, N., Smallwood, P. M., Macke, J. P. and Nathans, J. (2002). Frizzled-3 is required for the development of major fiber tracts in the rostral CNS. J Neurosci 22, 8563-8573.

Wang, Y., Zhang, J., Mori, S. and Nathans, J. (2006). Axonal growth and guidance defects in Frizzled3 knock-out mice: a comparison of diffusion tensor magnetic resonance imaging, neurofilament staining, and genetically directed cell labeling. J Neurosci 26, 355-364.

Wei, Y., Pattingre, S., Sinha, S., Bassik, M. and Levine, B. (2008). JNK1-mediated phosphorylation of Bcl2 regulates starvation-induced autophagy. Mol Cell 30, 678-688.

Weiss, L. A., Shen, Y., Korn, J. M., Arking, D. E., Miller, D. T., Fossdal, R., Saemundsen, E., Stefansson, H., Ferreira, M. A., Green, T., et al. (2008). Association between microdeletion and microduplication at 16p11.2 and autism. N Engl J Med 358, 667-675.

Westerlund, N., Zdrojewska, J., Padzik, A., Komulainen, E., Bjorkblom, B., Rannikko, E., Tararuk, T., Garcia-Frigola, C., Sandholm, J., Nguyen, L., et al. (2011). Phosphorylation of SCG10/stathmin-2 determines multipolar stage exit and neuronal migration rate. Nat Neurosci 14, 305-313.

Weston, C. R. and Davis, R. J. (2007). The JNK signal transduction pathway. Curr Opin Cell Biol 19, 142149. 
Whitmarsh, A. J., Cavanagh, J., Tournier, C., Yasuda, J. and Davis, R. J. (1998). A mammalian scaffold complex that selectively mediates MAP kinase activation. Science 281, 1671-1674.

Whittington, M. A. and Traub, R. D. (2003). Interneuron diversity series: inhibitory interneurons and network oscillations in vitro. Trends Neurosci 26, 676-682.

Wichterle, H., Alvarez-Dolado, M., Erskine, L. and Alvarez-Buylla, A. (2003). Permissive corridor and diffusible gradients direct medial ganglionic eminence cell migration to the neocortex. In Proc Natl Acad Sci U S A, pp. 727-732.

Wichterle, H., Garcia-Verdugo, J. M., Herrera, D. G. and Alvarez-Buylla, A. (1999). Young neurons from medial ganglionic eminence disperse in adult and embryonic brain. Nat Neurosci 2, 461-466.

Wichterle, H., Turnbull, D. H., Nery, S., Fishell, G. and Alvarez-Buylla, A. (2001). In utero fate mapping reveals distinct migratory pathways and fates of neurons born in the mammalian basal forebrain. Development 128, 3759-3771.

Williams, B. P. and Price, J. (1995). Evidence for multiple precursor cell types in the embryonic rat cerebral cortex. Neuron 14, 1181-1188.

Williams, T. A., Mars, A. E., Buyske, S. G., Stenroos, E. S., Wang, R., Factura-Santiago, M. F., Lambert, G. H. and Johnson, W. G. (2007). Risk of autistic disorder in affected offspring of mothers with a glutathione S-transferase P1 haplotype. Arch Pediatr Adolesc Med 161, 356-361.

Winchester, C. L., Ohzeki, H., Vouyiouklis, D. A., Thompson, R., Penninger, J. M., Yamagami, K., Norrie, J. D., Hunter, R., Pratt, J. A. and Morris, B. J. (2012). Converging evidence that sequence variations in the novel candidate gene MAP2K7 (MKK7) are functionally associated with schizophrenia. Hum Mol Genet 21, 4910-4921.

Wolfer, D. P., Henehan-Beatty, A., Stoeckli, E. T., Sonderegger, P. and Lipp, H. P. (1994). Distribution of TAG-1/axonin-1 in fibre tracts and migratory streams of the developing mouse nervous system. $J$ Comp Neurol 345, 1-32.

Woodward, N. D., Karbasforoushan, H. and Heckers, S. (2012). Thalamocortical dysconnectivity in schizophrenia. Am J Psychiatry 169.

Woolsey, T. and Van der Loos, H. (1970). The Structural Organization of Layer IV in the Somatosensory Region (SI) of Mouse Cerebral Cortex. The Description of a Cortical Field Composed of Discrete Cytoarchitectonic Units. Brain research 17.

Wright, A. G., Demyanenko, G. P., Powell, A., Schachner, M., Enriquez-Barreto, L., Tran, T. S., Polleux, F. and Maness, P. F. (2007). Close homolog of L1 and neuropilin 1 mediate guidance of thalamocortical axons at the ventral telencephalon. J Neurosci 27, 13667-13679.

Wu, C.-S., Ballester Rosado, C. J. and Lu, H.-C. (2011). What Can We Get From 'Barrels': The Rodent Barrel Cortex as a Model for Studying the Establishment of Neural Circuits. The European journal of neuroscience 34, 1663-1676.

Xu, B., Goldman, J. S., Rymar, V. V., Forget, C., Lo, P. S., Bull, S. J., Vereker, E., Barker, P. A., Trudeau, L. E., Sadikot, A. F., et al. (2010a). Critical roles for the netrin receptor deleted in colorectal cancer in dopaminergic neuronal precursor migration, axon guidance, and axon arborization. Neuroscience 169, 932-949.

Xu, P., Das, M., Reilly, J. and Davis, R. J. (2011). JNK regulates FoxO-dependent autophagy in neurons. Genes Dev 25, 310-322.

Xu, Q., Cobos, I., De La Cruz, E., Rubenstein, J. L. and Anderson, S. A. (2004). Origins of cortical interneuron subtypes. J Neurosci 24, 2612-2622.

Xu, X., Roby, K. D. and Callaway, E. M. (2010b). Immunochemical characterization of inhibitory mouse cortical neurons: three chemically distinct classes of inhibitory cells. J Comp Neurol 518, 389404. 
Yamasaki, T., Kawasaki, H., Arakawa, S., Shimizu, K., Shimizu, S., Reiner, O., Okano, H., Nishina, S., Azuma, N., Penninger, J. M., et al. (2011). Stress-activated protein kinase MKK7 regulates axon elongation in the developing cerebral cortex. J Neurosci 31, 16872-16883.

Yamasaki, T., Kawasaki, H. and Nishina, H. (2012). Diverse Roles of JNK and MKK Pathways in the Brain. J Signal Transduct 2012, 459265.

Yang, D. D., Kuan, C. Y., Whitmarsh, A. J., Rincon, M., Zheng, T. S., Davis, R. J., Rakic, P. and Flavell, R. A. (1997). Absence of excitotoxicity-induced apoptosis in the hippocampus of mice lacking the Jnk3 gene. Nature 389, 865-870.

Yau, H. J., Wang, H. F., Lai, C. and Liu, F. C. (2003). Neural development of the neuregulin receptor ErbB4 in the cerebral cortex and the hippocampus: preferential expression by interneurons tangentially migrating from the ganglionic eminences. Cereb Cortex 13, 252-264.

Yokota, Y., Gashghaei, H. T., Han, C., Watson, H., Campbell, K. J. and Anton, E. S. (2007). Radial glial dependent and independent dynamics of interneuronal migration in the developing cerebral cortex. PLoS One 2, e794.

Yoshida, M. and Colman, D. (2000). Glial-defined Rhombomere Boundaries in Developing Xenopus Hindbrain. The Journal of comparative neurology 424.

Yu, L., Alva, A., Su, H., Dutt, P., Freundt, E., Welsh, S., Baehrecke, E. H. and Lenardo, M. J. (2004). Regulation of an ATG7-beclin 1 program of autophagic cell death by caspase-8. Science 304, 1500-1502.

Zdrojewska, J. and Coffey, E. T. (2014). The impact of JNK on neuronal migration. Adv Exp Med Biol 800, 37-57.

Zechel, S., Nakagawa, Y. and Ibanez, C. F. (2016). Thalamo-cortical axons regulate the radial dispersion of neocortical GABAergic interneurons. Elife 5;e20770.

Zerucha, T. and Ekker, M. (2000). Distal-less-related homeobox genes of vertebrates: evolution, function, and regulation. Biochem Cell Biol 78, 593-601.

Zhang, D., Snyder, A. Z., Fox, M. D., Sansbury, M. W., Shimony, J. S. and Raichle, M. E. (2008). Intrinsic functional relations between human cerebral cortex and thalamus. J Neurophysiol 100, 1740 1748.

Zhang, D., Snyder, A. Z., Shimony, J. S., Fox, M. D. and Raichle, M. E. (2010). Noninvasive functional and structural connectivity mapping of the human thalamocortical system. Cereb Cortex 20, 11871194.

Zhang, F., Yu, J., Yang, T., Xu, D., Chi, Z., Xia, Y. and Xu, Z. (2016). A Novel c-Jun N-terminal Kinase (JNK) Signaling Complex Involved in Neuronal Migration during Brain Development. J Biol Chem 291, 11466-11475.

Zhou, L., Bar, I., Achouri, Y., Campbell, K., De Backer, O., Hebert, J. M., Jones, K., Kessaris, N., de Rouvroit, C. L., O'Leary, D., et al. (2008). Early forebrain wiring: genetic dissection using conditional Celsr3 mutant mice. Science 320, 946-949.

Zhou, L., Qu, Y., Tissir, F. and Goffinet, A. M. (2009). Role of the atypical cadherin Celsr3 during development of the internal capsule. Cereb Cortex 19 Suppl 1, i114-119.

Zhu, X., Raina, A. K., Rottkamp, C. A., Aliev, G., Perry, G., Boux, H. and Smith, M. A. (2001). Activation and redistribution of $\mathrm{c}$-jun $\mathrm{N}$-terminal kinase/stress activated protein kinase in degenerating neurons in Alzheimer's disease. J Neurochem 76, 435-441.

Zhu, Y., Li, H., Zhou, L., Wu, J. Y. and Rao, Y. (1999). Cellular and molecular guidance of GABAergic neuronal migration from an extracortical origin to the neocortex. Neuron 23, 473-485.

Ziats, M. N. and Rennert, O. M. (2011). Expression profiling of autism candidate genes during human brain development implicates central immune signaling pathways. PLoS One 6, e24691.

Zimmer, G., Garcez, P., Rudolph, J., Niehage, R., Weth, F., Lent, R. and Bolz, J. (2008). Ephrin-A5 acts as a repulsive cue for migrating cortical interneurons. Eur J Neurosci 28, 62-73. 